Bull. Mater. Sci., Vol. 21, No. 1, February 1998, pp. 1-70. (O Printed in India.

\title{
Techniques and applications of electron spin resonance*
}

\author{
C S SUNANDANA \\ School of Physics, University of Hyderabad, Hyderabad 500 046, India
}

MS received 27 January 1996

\begin{abstract}
A broad-spectrum review of the applications of electron spin resonance to advanced materials is presented. Starting with basic concepts the reader is taken through a quick tour of techniques including continuous-wave and pulse ESR, microscopy and imaging, as well as a few emerging techniques. Applications of spin identification, spin counting, spin mapping and spin imaging of a variety of advanced solid state materials including metals and alloys, semiconductors, inorganics, electroceramics, catalysts, intercalates, polymers, glasses, and organic charge-transfer complexes besides superionic conductors and high-temperature superconductors are included. It is thus demonstrated that the technique is at once specific, sensitive to composition, phase and texture yet accurate enough to be a quantitative but non-invasive tool that promises to be useful in the study of newer and newer materials including multilayers, ferrofluids and nanomaterials.
\end{abstract}

Keywords. Electron spin resonance; advanced materials; ESR microscopy and imaging; semiconductors; polymers; glasses; superionic; superconductors.

\section{Preamble}

The use of magnetic moment of an unpaired electron a fundamental particle with an intrinsic property of 'spin' (table 1) that is naturally found (or artificially created) in materials as an essential part of their unique crystal structures, and dictating their electrical, optical and magnetic behaviour - as a microscopic, resonance spectroscopic probe of characterization is the basis of electron spin resonance (ESR) or more generally speaking electron paramagnetic resonance (EPR). It is the hierarchy of interactions of this magnetic moment with its neighbouring and the more distant material environment that is exploited in this technique to learn more about either the materials processing per se or the induced process(es) that take place within the material. It could be said that the EPR technique and advanced materials are 'made for each other'.

Starting with a brief historical perspective, this article goes on to provide a brief account of the methods and applications of this technique. The spectrum of the advanced materials covered include semiconducting materials, polymer materials, ceramics and glasses, and optoelectronics and superionic materials. ESR imaging and microscopy as well as certain emerging techniques such as millimeter ESR are briefly discussed. The examples chosen are meant to be representative, and the seemingly inexhaustible information is available in standard books, review articles and papers referenced in this article.

*Dedicated to my teacher, the Late Prof. C Ramasastry (19231989)

\section{A brief historical perspective}

Electron spin resonance (ESR) or more generally speaking electron paramagnetic resonance (EPR), discovered by Zavoiskii (1944) in $\mathrm{MnSO}_{4}$ employing a $47.6 \mathrm{G} \mathrm{dc}$ magnetic field and a $133 \mathrm{MHz}$ rf magnetic field, is an extension of the original Stern-Gerlach experiment (Stern 1921; Gerlach and Stern 1924), on atomic beams which demonstrated the space quantization of atomic magnetic moments. In between, Rabi (1939a, b) had performed the 'nuclear Zeeman effect' experiment by using a radio frequency electromagnetic field perpendicular to a homogeneous dc magnetic field. Theoretically, the possibility of quantum transitions between magnetic sublevels of atoms under the influence of an external magnetic field was suggested by Einstein and Ehrenfest (1922).

The earliest applications of microwave resonance spectroscopic technique were on (i) $\mathrm{CuSO}_{4} \cdot 5 \mathrm{H}_{2} \mathrm{O}$ crystals in which 'exchange narrowing' was discovered (Bagguley and Griffiths 1950), (ii) F-centres in alkali halides from which structural information was obtained (Kip et al 1953), and (iii) donor atoms (P, As and $\mathrm{Sb}$ ) in silicon in which motional 'narrowing' and delocalization of donor electron wave functions were observed (Fletcher et al 1954), which paved the way for the discovery of electron-nuclear double resonance-ENDOR in phosphorus doped silicon (Feher 1959), which has been very profitably applied to the elucidation of defects in amorphous semiconductors. The EPR studies of surfaces also began with silicon (Fletcher et al 1954; Feher 1959; Brodsky and Title 1961; Roitsin and Maevskii 1989). 
Table 1. Properties of fundamental particles relevant for magnetic resonance spectroscopy.

\begin{tabular}{lcccc}
\hline Property & Electron & Proton & Neutron & Muon \\
\hline Charge (C) & $-1.602192 \times 10^{-19}$ & & 0 & \\
Mass (kg) & $9 \cdot 109534(47) \times 10^{-31}$ & $1.6726485(86) \times 10^{-27}$ & $1.6749543(86) \times 10^{-27}$ & $1.883566(11) \times 10^{-28}$ \\
$\begin{array}{l}\text { Magnetic moment } \\
\quad \text { Joule/Tesla) }\end{array}$ & $9.284832(36) \times 10^{-24}$ & $1.401671(51) \times 10^{-27}$ & & $4.490474(18) \times 10^{-26}$ \\
& & $2.7923456(11) \mu_{\mathrm{N}}$ & $-1.91304184(88) \mu_{\mathrm{N}}$ & \\
$g$-factor (Zeeman) & 2.00231929 & $\mu_{\mathrm{N}}=5.0505 \times 10^{-27} \mathrm{~J} / \mathrm{T}$ & $1.00116616(0.31)$ \\
$g$-factor (spin-orbit) & 2.00463858 & & & \\
\hline
\end{tabular}

Sources:

1. Weast R C (ed.) 1988 CRC Handbook of Physics and Chemistry 1st Student edn.

2. Krane K S 1988 Introduction to Nuclear Physics (New York: Wiley).

An investigation of permeability of ferromagnetic metals $\mathrm{Fe}, \mathrm{Co}$ and $\mathrm{Ni}$, by Griffiths (1946) led to the discovery of ferromagnetic resonance. An examination of free radicals in leaves, seeds and tissue preparation saw the first biological application (Commoner et al 1954). McConnell pioneered the use of 'spin labels' or free radical substituents in biological systems (Stone et al 1965), while Sands (1955) pioneered a continuing structural investigation on transition metal ion-doped glasses. Weeks (1956) applied the technique for studying radiationinduced centres in crystalline quartz, and, Yasaitis and Smaller (1953) investigated the paramagnetic centres in irradiated borate glasses. The studies on mechanically produced free radicals in polymers were pioneered by Zakrevskii et al (1968).

The observation of electron spin echoes (Gordon and Bowers 1958; Mims et al 1961) ushered in the era of pulsed electron spin resonance, coming in the wake of Hahn's discovery of nuclear spin echoes (Hahn 1950). The last decade has seen the emergence of EPR imaging and microscopy (Ikeya 1991), again following the discovery of NMR imaging (Lauterbur 1973).

The bludgeoning activity in this field is evidenced by the very recent appearance of a number of comprehensive monographs and workshop proceedings (Pilbrow 1990; Yordanov 1991; Mabbs and Collison 1992; Ikeya 1993), besides the existing classics (Wertz and Bolton 1972; Atherton 1973; Abragam and Bleaney 1989).

\section{ESR concepts}

The phenomenon of resonance absorption of the magnetic component of external electromagnetic radiation by a paramagnetic or spin system such as a hydrogen atom in silicon, or an irradiated polymer or cobalt in barium titanate is based on the existence or postulation of: (i) intrinsic magnetic moments due to 'spins' of electrons $\left(\mu_{\mathrm{e}}\right)$ and certain nuclei $\left(\mu_{\mathrm{l}}\right)$ (table 2); (ii) gyroscopic motion of these moments and an angular momentum thereof; (iii) quantization of these angular momenta $S$ and $I$ for electron and nuclei, respectively; (iv) distinct magnetic energy levels created by an externally applied static magnetic field and a separation between these levels; (v) a substantial number of 'spins' in their lowest energy states at ambient temperatures according to Boltzmann distribution law, according to which the population difference between two energy levels at temperature $T$ is proportional to the negative exponential of ratio of the difference between concerned energies and the thermal energy $k_{\mathrm{B}} T$, where $k_{\mathrm{B}}$ is the Boltzmann constant, and finally, (vi) the natural precession frequency called the Larmor frequency for the spin system (which for the electronic moment depends on the external magnetic field and for the nuclear moment on the internal electronic field) which may be approached either by scanning the frequency of the alternating field at a fixed static magnetic field or by scanning the static magnetic field at a fixed frequency.

\subsection{Classical picture}

A planetary model of an atom with a magnetic nucleus $\left(\mu_{1} \neq 0\right)$ and an unpaired electron $\left(\mu_{\mathrm{e}} \neq 0\right)$ illustrates their intrinsic property of 'spin' in a classical way (figure 1). An electron of spin $S$ with a magnetic moment $\mu_{\mathrm{e}}$ experiences - much like a tiny bar magnet - a torque $\mu \times H_{0}$ in an external magnetic field. This angular motion constantly and continuously changes direction so that

$$
\frac{\mathrm{d}(S \hbar)}{\mathrm{d} t}=\tau=\mu \times H_{0}
$$

$\tau$ being always normal to the $\mu-H_{0}$ plane, changes only the direction of $\mu$ as the latter precess around $H_{0}$ with a frequency

$$
\omega_{0}=\gamma_{\mathrm{e}} H_{0},
$$


where $\gamma_{\mathrm{e}}=$ electron magnetogyric ratio $=-g e / 2 m c$, with $g$ being the spectroscopic splitting factor, $e$ the electron charge, $m$ the electron mass (see table 2) and $c$ the velocity of light. For the electron, the dipole moment opposes the angular momentum so that

$$
\mu=-\gamma \hbar S .
$$

The magnetic component of the electromagnetic field (which lies in microwave region for ESR and radiofrequency region for nuclear magnetic resonance or NMR), $\left|H_{1}\right|\left(\ll H_{1}\right)$ also rotates with microwave frequency $\omega$ and exerts a torque $\mu \times H_{1}$ on $\mu$. As long as $\omega \neq \omega_{0}$ this torque is zero on the average but when $\omega=\omega_{0}, \mu$ precesses around $H_{1}$ with a frequency $\omega_{1}=\gamma_{e} H_{1}$ besides its usual precession around $H_{0}$ with frequency $\omega_{0}\left(\gg \omega_{1}\right)$. Consequently, $\mu$ slowly changes its direction flipping down and eventually reaching a position opposite to the original orientation (figure 2). In the flipping process work is done on the dipole moment by the microwave magnetic field. In other words, the system absorbs energy from the microwave field $H_{1}$ during resonance. At resonance the oscillatory magnetic moment, normal to $H$, produced by Larmor precession interacts with the small oscillatory magnetic field $H_{1} \cos \omega t$, also normal to $H$, and changes the direction of component of $\mu$ along $H$ by $180^{\circ}$, and thus changes the energy of the electronic dipole, causing ESR absorption. Thus at resonance,

$$
\begin{aligned}
v=v_{0} & =g(e / 2 m) H(2 \pi) \\
& =139.96(g H)(v \text { in } \mathrm{Hz}, H \text { in } \mathrm{T}) .
\end{aligned}
$$

$H$ is also expressed in Oersted and gauss, with 10,000 such units to a Tesla.

\subsection{Quantum picture}

Quantum mechanically, the phenomenon of electron paramagnetic resonance is described as the magnetic dipole transitions brought about by the interaction of the magnetic field of the microwave radiation with a magnetic moment in the absorbing system. Quantum mechanics helps in arriving at the probability of these transitions and shows that this probability has a sharp maximum when $\omega=2 \mu_{\mathrm{B}} H_{0} / \hbar$ or $h v=2 \mu_{\mathrm{B}} H_{0}$, where $\mu_{\mathrm{B}}$ is the Bohr magneton. In other words, $\omega$ is $\omega_{j}$, the Larmor frequency, so that the probability for the absorption of microwave power by the system is maximum when $H_{1}$ is rotating at the Larmor frequency. For a spin system with two energy levels, this probability is given by (Atherton 1973):

$$
P_{12}=\frac{4 \mu_{\mathrm{B}} H_{1}^{2}}{\hbar^{2}}\left\{\frac{\sin ^{2} 1 / 2\left(2 \mu_{\mathrm{B}} H_{0} / \hbar-\omega\right) \hbar t}{2 \mu_{\mathrm{B}} H_{0} / \hbar-\omega^{2}}\right\},
$$

when the total magnetic field acting on the spin system is the vector sum

$$
\mathbf{H}=H_{1}(i \cos \omega t+j \sin \omega t)+H_{0} k,
$$

and $g_{\mathrm{e}}$ is assumed to be 2 . The resonance condition can be arrived by considering the allowed energies of the electron magnetic dipole

$$
\mu\left[|\mu|=\gamma \hbar S(S+1)^{1 / 2}\right],
$$

in the field $H_{0}$, using the energy operator

$$
\mathcal{H}=-\mu \cdot H_{0}=\gamma \hbar S \cdot H_{0} .
$$

Taking $H_{0}$ along $+z$ axis (figure 2) the allowed energies are

$$
E_{\mathrm{M}_{\mathrm{s}}}=\gamma \hbar H_{0} M_{\mathrm{s}}
$$

where the quantum number $M_{\mathrm{s}}$ specifies the allowed values of the z-components of $S$ viz. $-S,-S+1$ $\ldots S-1, S$. For $S=1 / 2, M_{s}= \pm 1 / 2$ and the allowed energies are

$$
E \pm \frac{1}{2}= \pm \gamma_{c} \hbar H_{0}
$$

Transitions between these levels (figure 3 ) caused by magnetic dipole radiation require that $\Delta M_{\mathrm{s}}= \pm 1$. The resonance condition that the energy quantum $\hbar \omega$ of $H_{1}$ must satisfy is

$$
\hbar \omega=E \pm \frac{1}{2}-E-\frac{1}{2}=\gamma_{\mathrm{e}} \hbar H_{0},
$$

which coincides with the classical picture $\omega=\gamma H_{0}=\omega_{0}$. The basic gyromagnetic ratio for the free electron is

$$
\gamma_{\mathrm{e}}=2 \pi(28.0246 \mathrm{GHz} / T) \text {. }
$$

\subsection{Spin relaxation}

The above pictures are valid for the hypothetical case of a single isolated spin or a paramagnet. In the real world materials contain a large number of spins, so that the concept of a 'reservoir' or a 'bath' or the concept of a 'thermal equilibrium' comes into the picture. The reservoir (a crystal lattice or a glassy/polymer matrix) can take energy from the spin system when the latter makes the transition to the upper of the two states $M_{\mathrm{s}}= \pm 1 / 2$.

The populations of spins $N^{+}$and $N^{-}$, present in the two energy states $M_{\mathrm{s}}=+1 / 2$ and $M_{\mathrm{s}}=-1 / 2$ respectively, when the spin system is in thermal equilibrium with the reservoir are governed by the Boltzmann distribution

$$
N^{+} / N^{-}=\exp \left(-\gamma \hbar H_{0} / k_{\mathrm{B}} T\right) .
$$




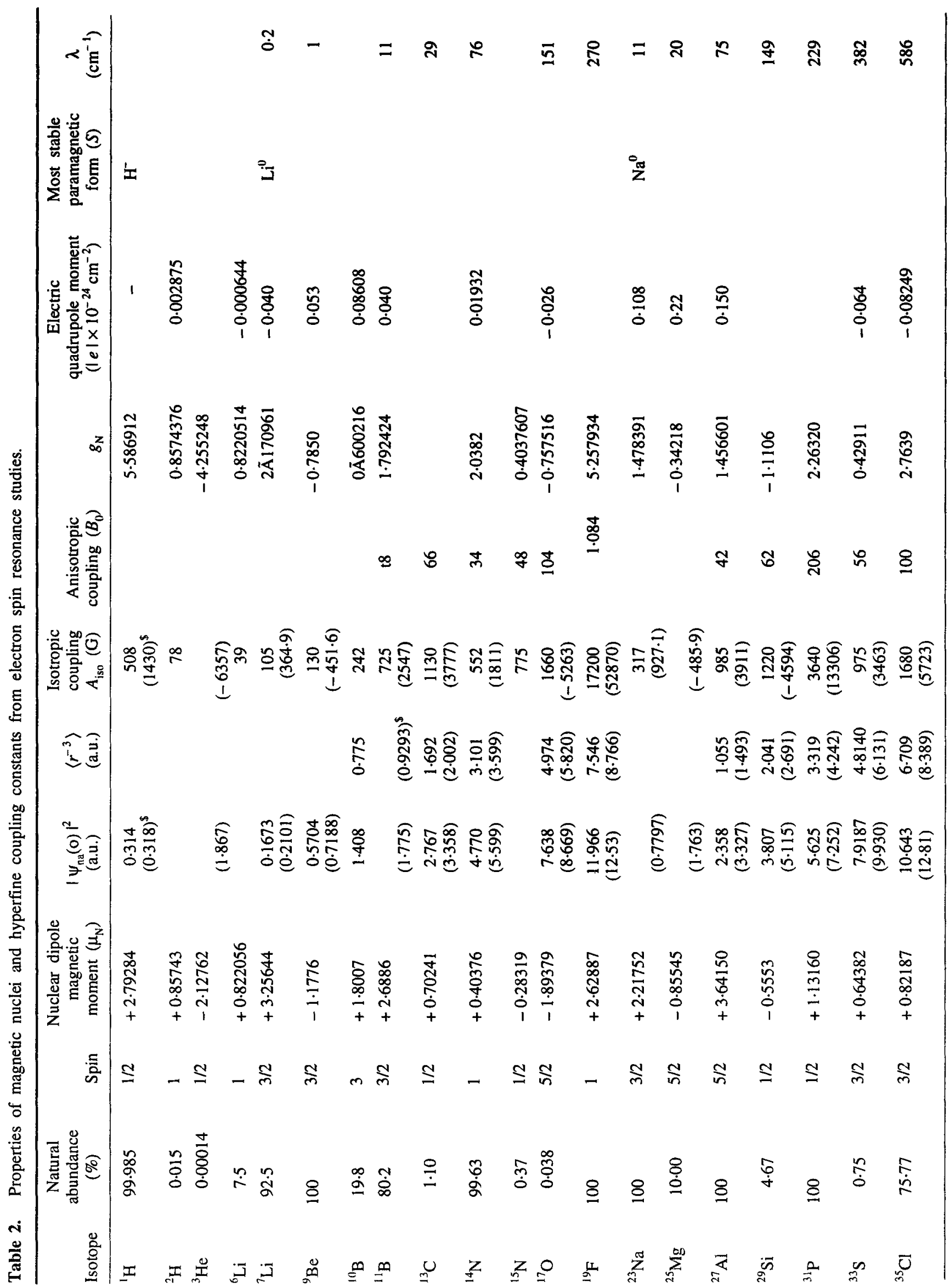




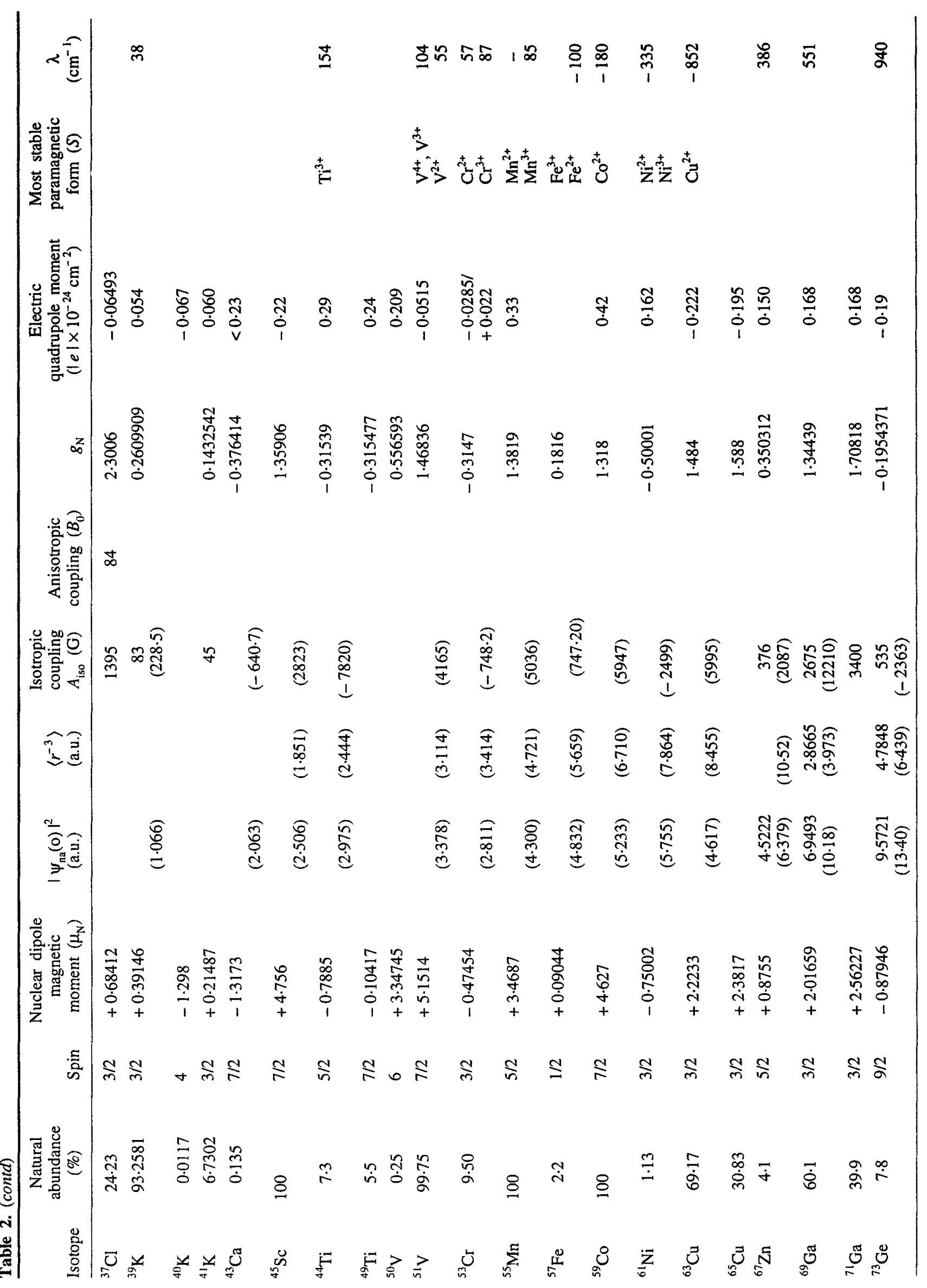




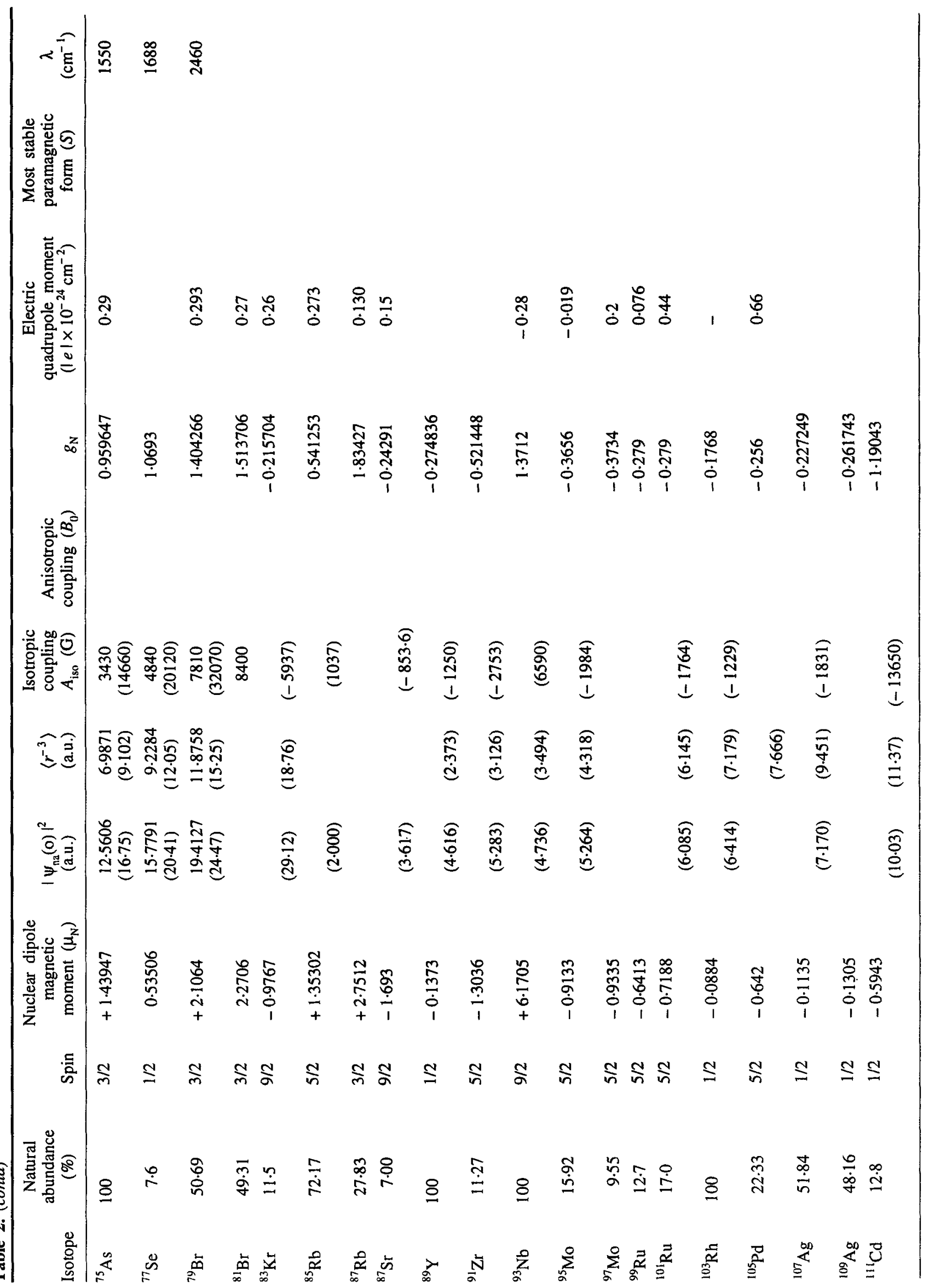




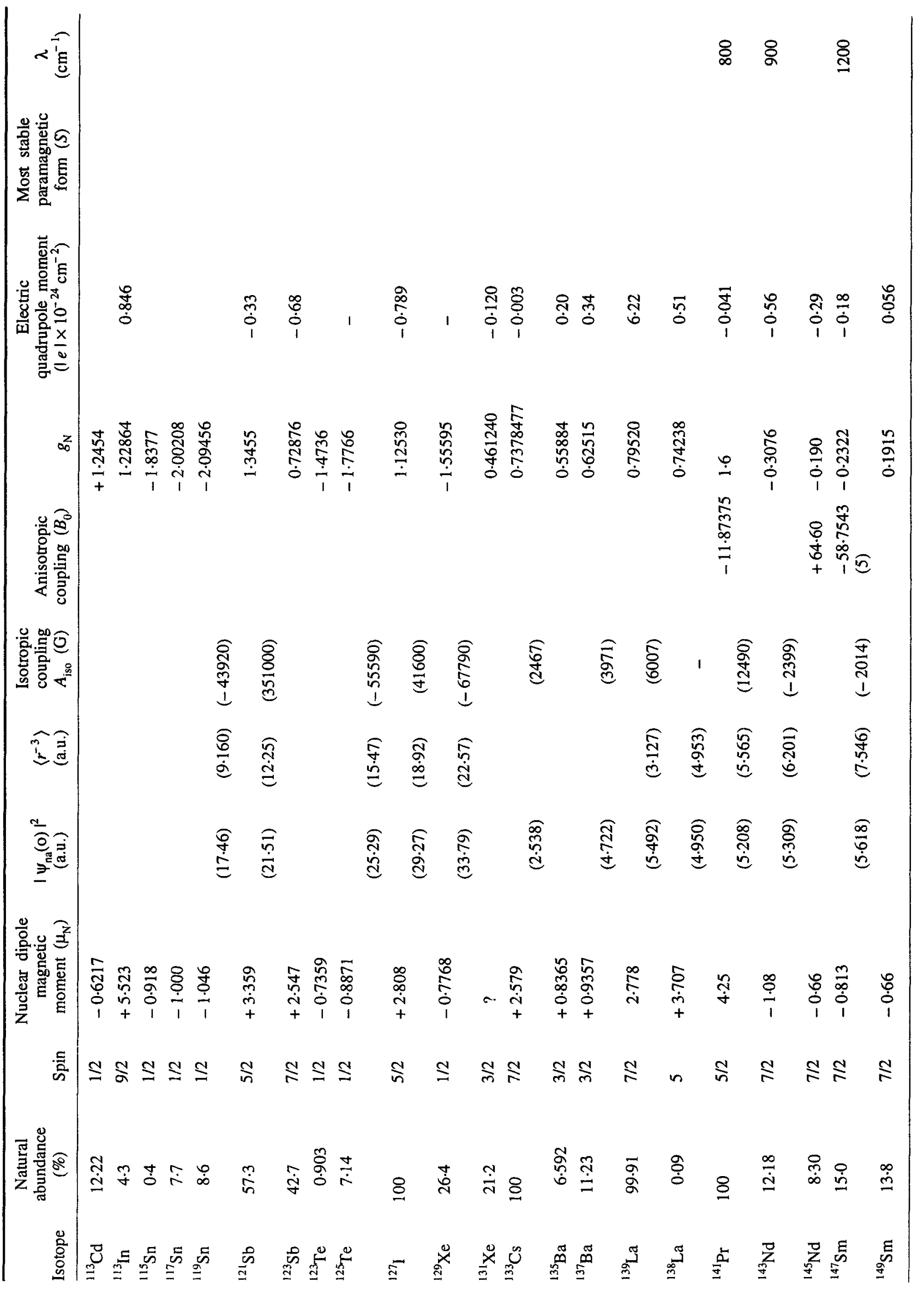




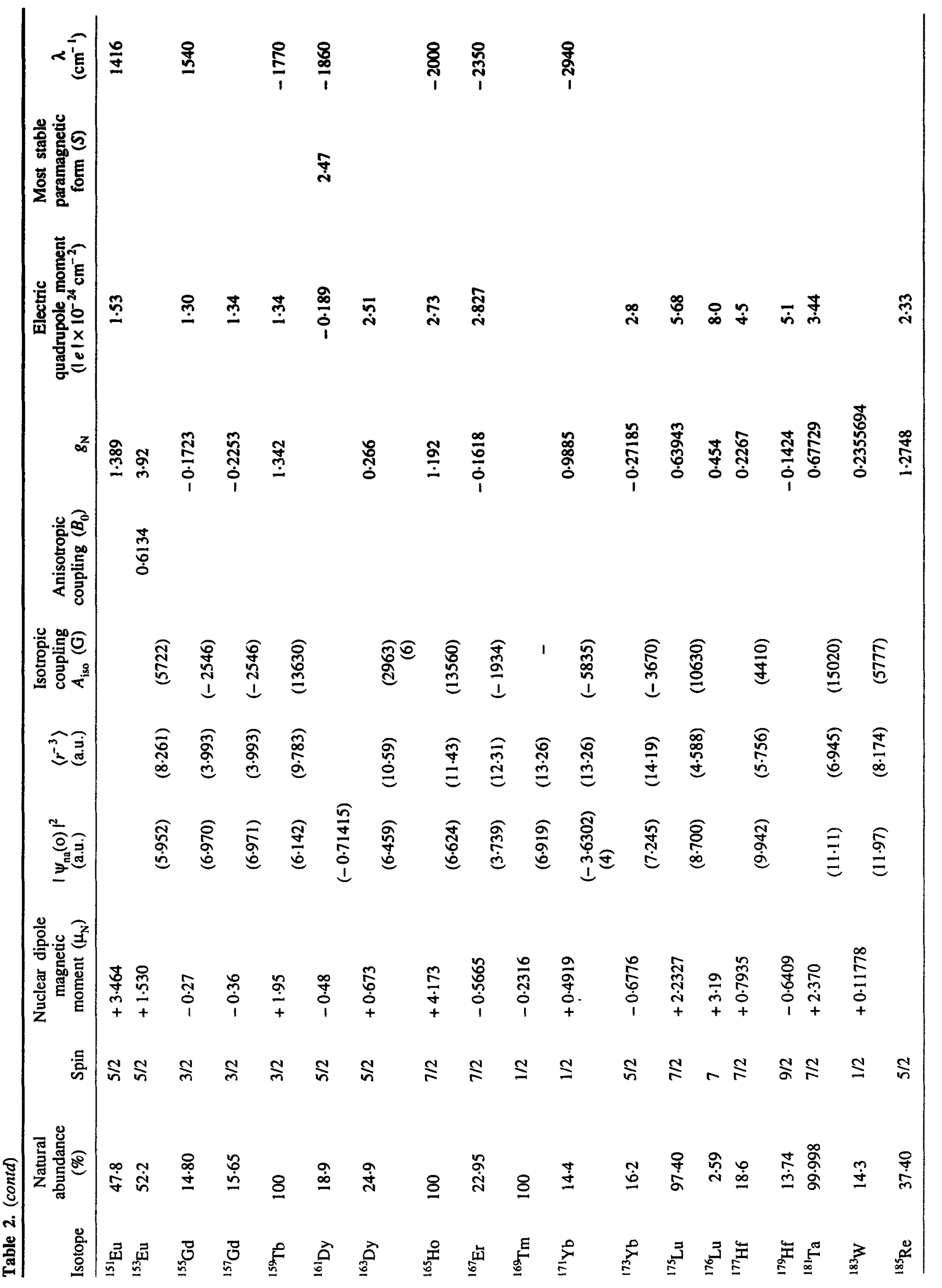


Techniques and applications of electron spin resonance

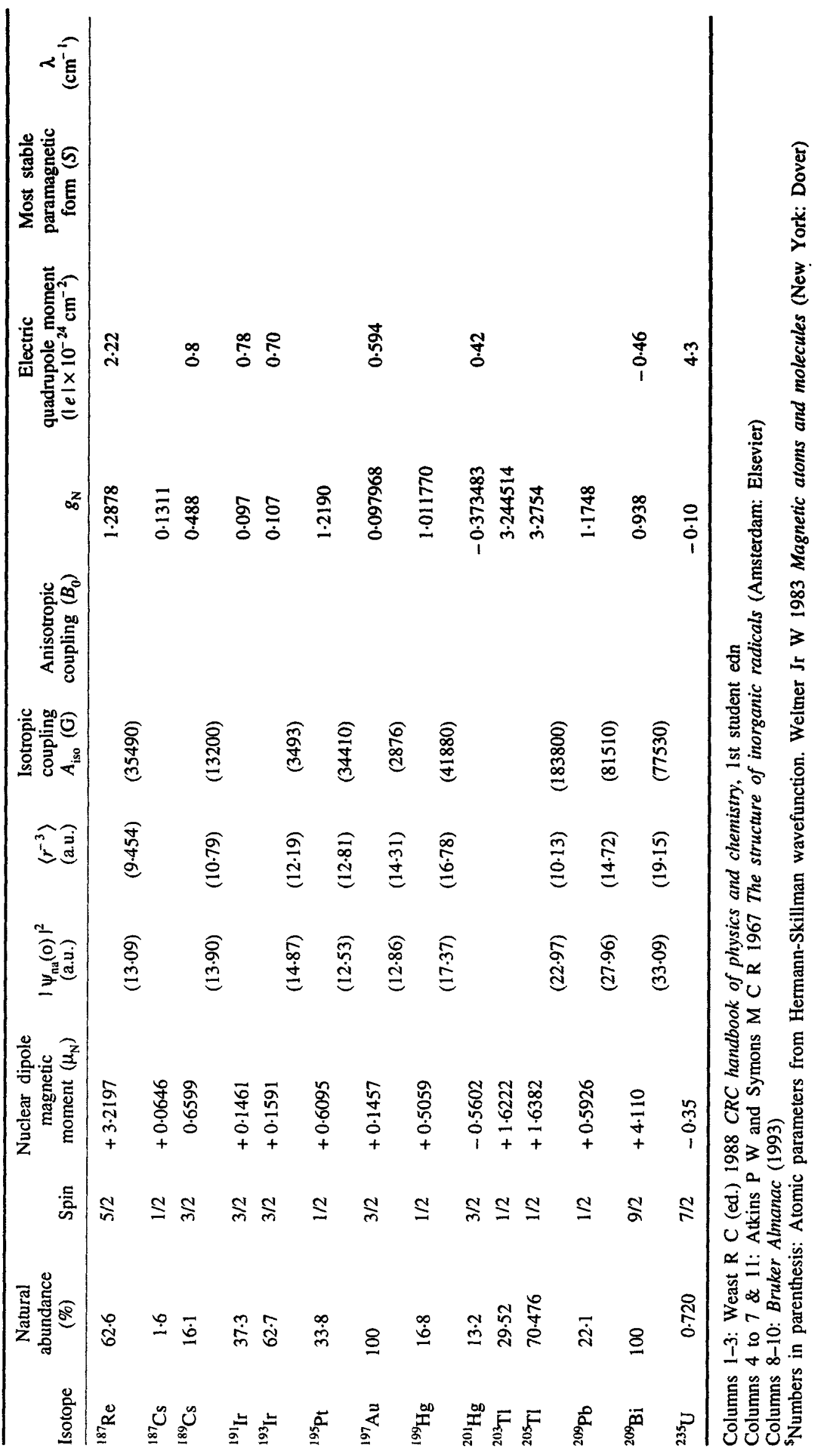


Equation (11) ensures that there is always an excess spin population in the ground state ready to undergo transitions. More significantly, since the $N^{-}>N^{+}$energy absorption would occur until $N^{-}=N^{+}$, when the spin system would become saturated and no resonance would be detectable. In fact, there are two mechanisms acting within the material by which energy is effectively transferred by the spin system to the surroundings. The spin-lattice relaxation, characterized by time $T_{1}$ and exponential in time, results from interactions of the electronic magnetic moments with each other and with the other electrons of the host material, or the 'lattice'. This 'longitudinal' relaxation causes changes in the component of $\mu$ parallel to $H_{0}$. A short $T_{1}$ affects the linewidth of the resonance through the energy-time uncertainty relation $\Delta E \Delta \tau \geq \hbar, \Delta E$ being the uncertainty of an energy level and $\Delta \tau$ being the lifetime of that state, i.e. $T_{1}$. A very short $T_{1}$ would thus imply a large $\Delta E$ and a broadened ESR line at ambient. ESR lines can be broadened by magnetic interaction among the spins themselves, as a result of which the different spins would experience slightly different local fields in the z-axis, leading to a spread in the Larmor precession frequencies. Eventually the spins would not precess in phase at all, and there would be a gradual dephasing, exponential in time, the process being characterized by a transverse or a spin-spin relaxation time $T_{2}$. Spin-spin relaxation processes are adiabatic because there is no exchange of energy between the spin system and the reservoir.

Thus the spin relaxation mechanisms are crucial to the observation of electron spin resonance spectra. Aspects of lineshape and linewidth are considered in $\$ 6$.

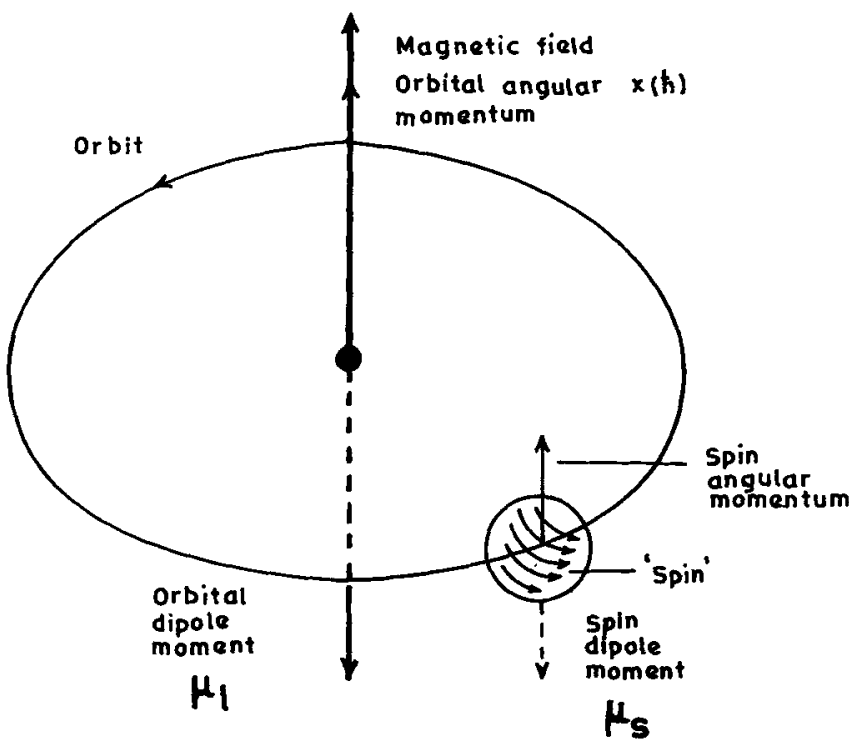

Figure 1. Planetary model for electron motion in an applied magnetic field. 'Orbiting' and 'spinning' gives the electron the orbital and spin angular momenta, $\mu_{1}$ and $\mu_{\mathrm{s}} . \mu_{1}=0$ for $s$-electrons and $\mu_{1} \neq 0$ for $p, d$ and $f$ 'paramagnetic' electrons in a material.

\section{ESR and allied phenomena}

\subsection{Electron spin resonance}

When a material containing electron magnetic dipoles is placed in a static magnetic field and subjected to electromagnetic radiation, absorption attributable to magnetic dipole transitions occurs at one or more characteristic frequencies in the microwave region of the electromagnetic spectrum.

For a system with electron spin $S$ (ex: $\mathrm{H}$ atom in silicon $(S=1 / 2)$ ) there are $2 S+1$ energy levels in a static magnetic field $H$ given by:

$$
E=\left\langle\psi_{\mathrm{i}}|\hat{\mathcal{H}}| \psi_{\mathrm{i}}\right\rangle=M_{\mathrm{s}} g \mu_{\mathrm{B}} H,
$$

where $\psi_{\mathrm{i}}$ is a characteristic wave function of the $z$ -

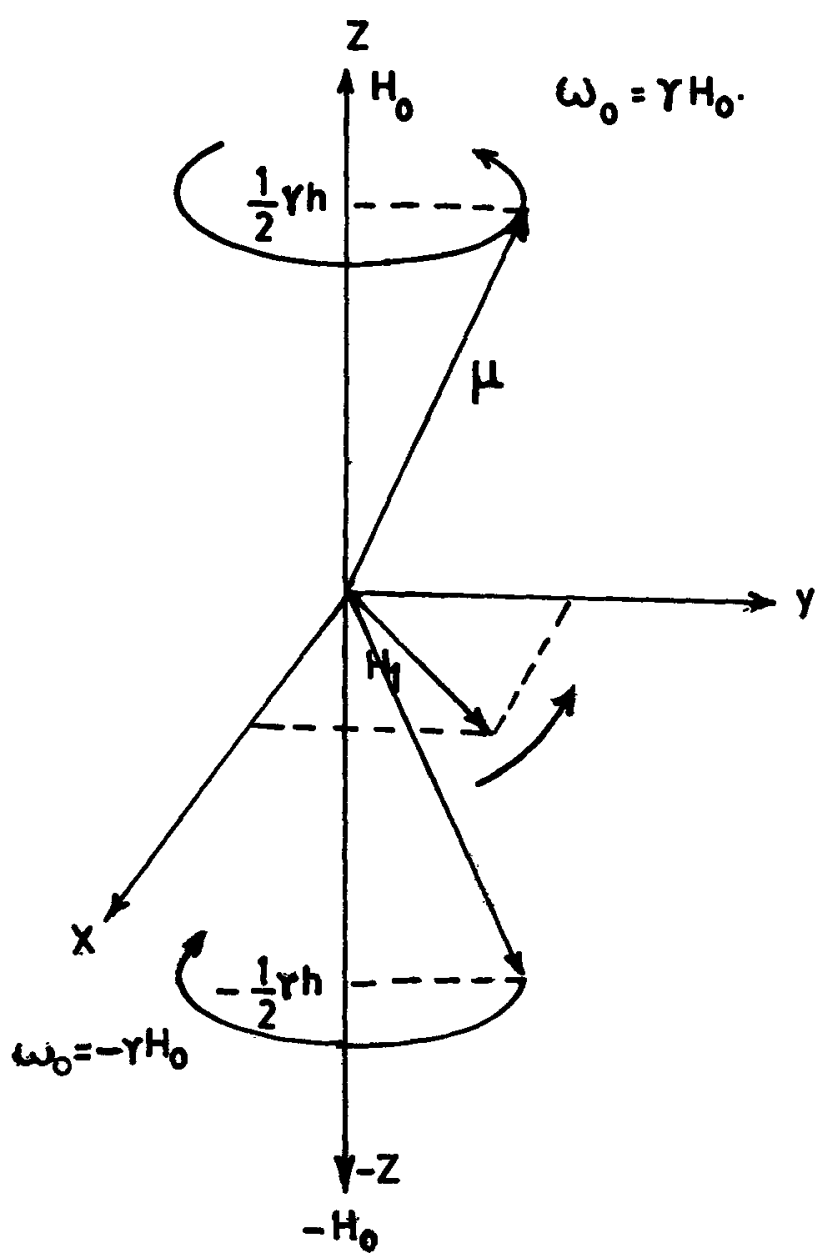

Figure 2. Larmor precession of an electron spin $(S=1 / 2)$ magnetic moment $(\mu)$ in an applied magnetic field $H_{0}$ with a frequency $\omega_{0}=\gamma H_{0}$ along z-axis. An oscillatory magnetic field $H_{1}\left(\ll H_{0}\right)$ at microwave frequency applied normal to $H_{0}$ 'tips', the moment and changes the sense of precession $\omega_{0}=\gamma H_{0}$ and causes electron spin/paramagnetic resonance absorption, depicted as the 'cone inversion' through the origin. 
component of the spin angular momentum $S_{\mathrm{z}} \hbar$, and $\hat{\mathcal{H}}$ is the spin Hamiltonian operator

$$
\hat{\mathcal{H}}=g \mu_{\mathrm{B}} H \hat{S}_{\mathrm{z}},
$$

$\mu_{\mathrm{B}}$ or the Bohr magneton is a unit for the magnetic moment of the electron $\mu_{\mathrm{e}}=1 / 2 g_{\mathrm{e}} \mu_{\mathrm{B}}=9.28485 \times 10^{-21}$ $J T^{-1}$ and the free electron $g$-value is 2.00232 . The magnetic dipole transitions between the Zeeman levels (so-called because the dc magnetic field separates the levels as in Zeeman effect observed in atomic spectra) are induced by the magnetic component of the microwave electromagnetic field, when the energy of the microwave photon matches the energy level separation

$$
\Delta E=h v=\Delta M_{\mathrm{s}} g \mu_{\mathrm{B}} H_{\mathrm{r}}
$$

where $\Delta M_{\mathrm{s}}= \pm 1$ is the selection rule for ESR (figure 4). Equation (14) implies a reorientation of the electron magnetic moment, or a flipping which is possible only when the electromagnetic field is polarized such that the oscillating magnetic field has a component perpendicular to the static magnetic field.

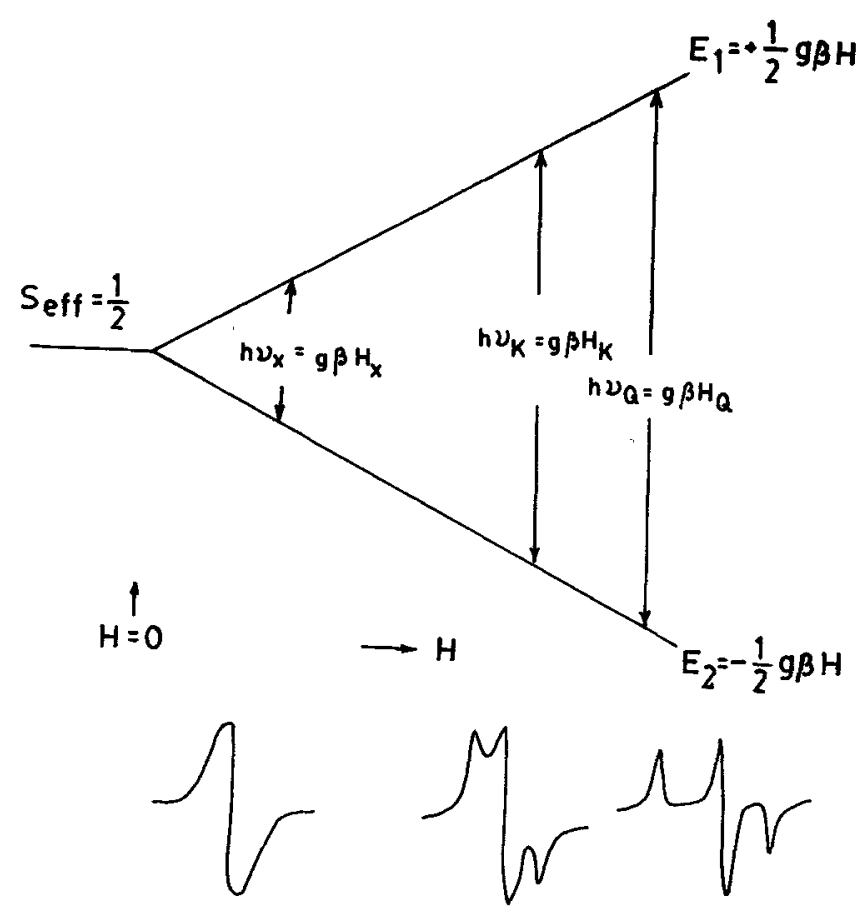

Figure 3. ESR transitions for a system with an effective spin $1 / 2$ in progressively increasing dc magnetic fields, for $g=2$, $H_{\mathrm{x}}(0.339 T), H_{\mathrm{K}}(0.893 T)$ and $H_{\mathrm{Q}}(125 \mathrm{~T})$ and corresponding higher microwave frequencies $v_{x}$ : X-band $(9.5 \mathrm{GHz})\left(v_{\mathrm{K}}: \mathrm{K}\right.$-band $(25 \mathrm{GHz})$ and ${ }_{\mathrm{Q}}:$ Q-band $\left.(35 \mathrm{GHz})\right)$. The corresponding anisotropic ESR spectra of a polycrystalline sample are shown at the bottom to illustrate the improvement in resolution upon increase of microwave frequency.
The g-factor of (13) is in general a tensor, so that

$$
\hat{\mathcal{H}}=\mu_{\mathrm{B}} \hat{S} \cdot \tilde{g} \cdot \hat{H},
$$

or $\hat{\mathcal{H}}=\mu_{\mathrm{B}}\left(S_{\mathrm{x}} S_{\mathrm{y}} S_{\mathrm{z}}\right)\left[\begin{array}{lll}g_{\mathrm{xx}} & g_{\mathrm{xy}} & g_{\mathrm{xz}} \\ g_{\mathrm{yx}} & g_{\mathrm{yy}} & g_{\mathrm{zy}} \\ g_{\mathrm{zx}} & g_{\mathrm{zy}} & g_{\mathrm{zz}}\end{array}\right]\left[\begin{array}{c}H_{\mathrm{x}} \\ H_{\mathrm{y}} \\ H_{\mathrm{z}}\end{array}\right]$,

where $S_{\mathrm{x}}, S_{\mathrm{y}}, S_{\mathrm{z}}$ and $H_{\mathrm{x}}, H_{\mathrm{y}}$ and $H_{\mathrm{z}}$ are the components of $\hat{S}$ and $\hat{H}$, measured with respect to an arbitrary set, $\mathrm{x}, \mathrm{y}, \mathrm{z}$ of mutually orthogonal axes in the monocrystalline materials. The tensor components $g_{i j}$ refer to the $g$-value along the $\mathrm{j}$-axis when the magnetic field is applied along

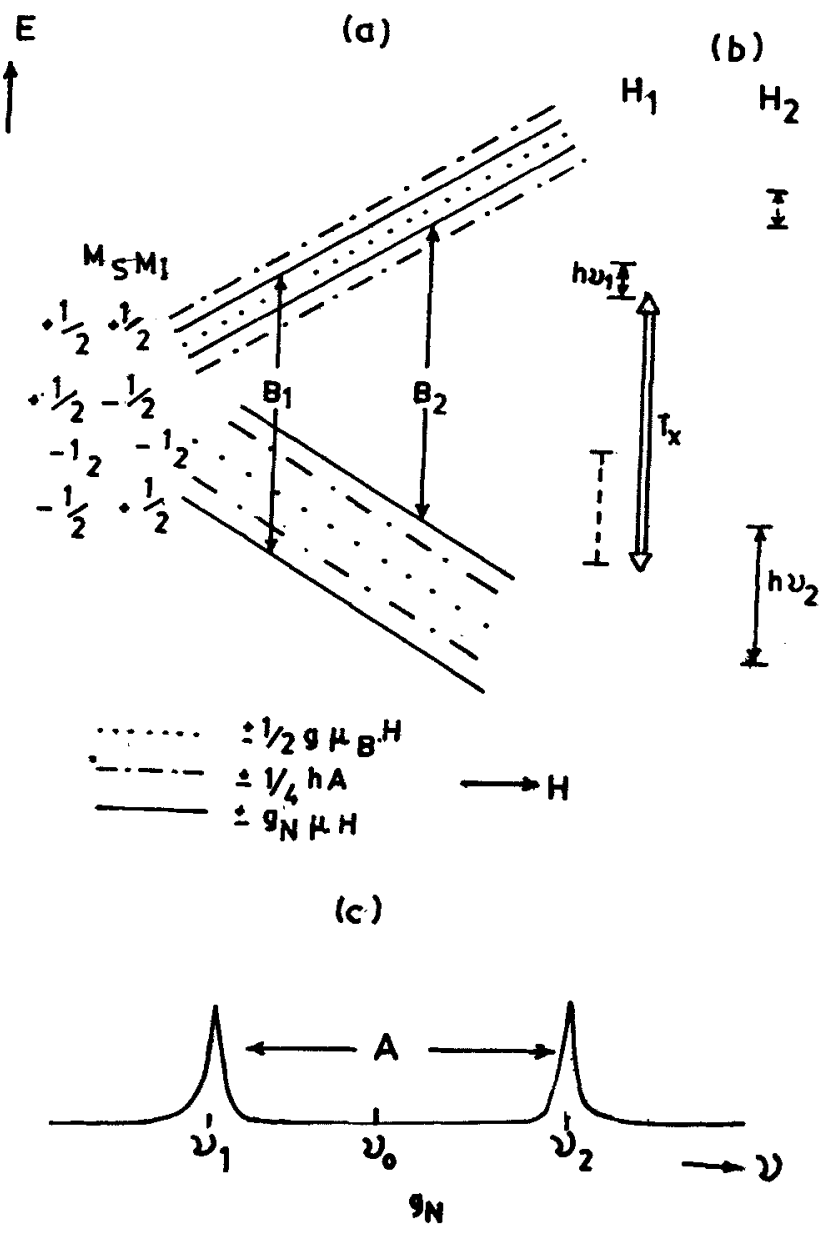

Figure 4. a. Energy levels for a spin system with $S=1 / 2$, $I=1 / 2$ as a function of applied magnetic field considering the electron Zeeman $(. .$.$) , hyperfine (-...) and nuclear Zeeman$ (-) effects. Two ESR transitions at $H_{1}$ and $H_{2}$ are observed. b. saturation of ESR transitions makes possible nuclear transitions at energies $w_{1}$ and $h w_{2}$ giving ENDOR transitions at $H_{1}$ and $H_{2}$, when a single 'cross-relaxation' $T_{x}$ operates between nuclear and electronic levels and c. expected 'ENDOR' spectra at frequencies $v_{1}$ and $v_{2}$. Separation gives $A$ the hyperfine coupling constant and $\nu_{0}$, the centre of gravity gives $g_{N}$-value (Wertz and Bolton 1972). 
the $\mathrm{i}$-axis. Many nuclei ( $\mathrm{H}$ through $\mathrm{Bi}$ in the periodic table of elements, see table 2) also possess an intrinsic spin angular momentum $I \hbar$ which produces an intrinsic magnetic field of the electron $H_{\mathrm{e}}$ that adds on to $H_{\mathrm{r}}$ so that the new resonance condition becomes

$$
h \nu=\left(H_{\mathrm{r}}+H_{\mathrm{e}}\right) g \mu_{\mathrm{B}} .
$$

For a nucleus such as ${ }^{1} \mathrm{H}$ or ${ }^{31} \mathrm{P}, l=1 / 2$ so that in the applied field $H$ the nucleus may take up one of the two configurations $M_{1}=+1 / 2$ or $M_{1}=-1 / 2$, where $M_{\mathrm{t}}$ is the z-component of the nuclear spin quantum number. The two applied fields at which resonance will occur are:

$$
H=h v / g \mu_{\mathrm{B}}=H_{0} \pm H_{\mathrm{e}}=H_{0}-a M_{\mathrm{I}} .
$$

Thus ESR is split into two, or, in general $(2 l+1)$ line 'hyperfine structure', $a$ being the hyperfine splitting constant. The origin of the hyperfine field $H_{e}$ is traced to the nonzero probability of the unpaired electron being found at the nucleus. This probability is maximum for electrons in s-orbitals, where wave functions are isotropic. The mutual magnetic interaction between the electron and the nuclear dipoles gives rise to the anisotropy of the hyperfine interaction.

Including the hyperfine interaction, as also the nuclear Zeeman interaction and the electric quadrupole interaction, (15a) becomes

$$
\hat{\mathcal{H}}=\mu_{\mathrm{B}} \hat{S} \cdot \tilde{g} \hat{H}+h \hat{S} \cdot \tilde{A} \tilde{I}-g_{\mathrm{N}} \mu_{\mathrm{N}} \hat{I} \cdot \hat{H}+h \hat{I} \cdot \tilde{Q} \hat{I},
$$

where $I_{\mathrm{N}}$ and $\mu_{\mathrm{N}}$ are the nuclear $g$-factor and nuclear Bohr magneton respectively, and, $\widetilde{A}$ and $\widetilde{Q}$ are the hyperfine and quadrupolar interaction tensors, symmetric and diagonalizable. In the next section, the $g$ - and $A$-tensors are related to physical quantities. Equation (17) is the spin Hamiltonian (Abragam and Bleaney 1989) - generally a polynomial in $S_{\text {eff }}$ the effective spin angular momentum - where $2 S_{\text {eff }}+1$ equals the total number of electronic states whose properties are being described and which may be used to determine the energy levels of the spin system and thus deduce the ESR transition frequencies. Therefore, the spin Hamiltonian such as (17) makes the contact between theory and experiment.

Considering the case of a spin-half nucleus containing an unpaired electron $(S=I=1 / 2)$ with (i) both electron Zeeman and magnetic hyperfine interaction isotropic, (ii) the static magnetic field $H$ taken along the $Z$-axis, $\mathcal{H}$ of (17) becomes

$$
\hat{\mathcal{H}}=g \mu_{\mathrm{B}} \hat{H} \hat{S}_{\mathrm{z}}+a \hat{S}_{\mathrm{z}} \hat{l}_{\mathrm{z}}+g_{\mathrm{N}} \mu_{\mathrm{N}} \hat{H} \hat{I}_{\mathrm{z}}
$$

where $a$ is the isotropic hyperfine coupling constant (measured in $\mathrm{Hz}$ ). The four possible combinations of $M_{s}$ and $\left.\left.\left.m_{1}: \quad 1+\frac{1}{2},+\frac{1}{2}\right\rangle, \quad 1+\frac{1}{2},-\frac{1}{2}\right\rangle, \quad 1-\frac{1}{2},+\frac{1}{2}\right\rangle$ and $\left|-\frac{1}{2},-\frac{1}{2}\right\rangle$ define the four spin states whose energies are given by:

$$
\begin{aligned}
E & \left.=M_{\mathrm{s}}, m_{\mathrm{l}}|\hat{\mathcal{H}}| M_{\mathrm{s}}, m_{\mathrm{l}}\right\rangle \\
& =M_{\mathrm{s}} g \mu_{\mathrm{B}} H+M_{\mathrm{s}} m_{\mathrm{l}} h a-m_{\mathrm{l}} g_{\mathrm{N}} \mu_{\mathrm{N}} H .
\end{aligned}
$$

Figure 4 shows these levels as a function of $H$. The ESR selection rule $\Delta M_{\mathrm{s}}= \pm 1$ corresponds to a change in the spin angular momentum of $\hbar$. During this transition, and in the presence of hyperfine structure, $m_{\mathrm{I}}$ must not change so that the total angular momentum of the nuclear spin and the electron spin system are conserved. This implies the extended selection rule $\Delta M_{s}= \pm 1, \Delta m_{1}=0$. The resulting ESR transitions are observed when

$$
h v=g \mu_{\mathrm{B}} H_{0} \pm \frac{1}{2} h a .
$$

From (16) and (14b), $A_{\text {iso }}=h a / g \mu_{\mathrm{B}}$ is the actual isotropic hyperfine interaction (see $\S 5$ ).

ESR measures ' $g$ ' and hyperfine coupling constants but does not measure $g_{\mathrm{N}}$ because the nuclear Zeeman energy does not normally affect the transition energies. Thus the unambiguous identification of the particular nucleus is generally impossible from ESR. But it is possible to resolve the ambiguity by simultaneously establishing ESR $\left(\Delta M_{\mathrm{s}}= \pm 1, \Delta m_{1}=0\right)$ and nuclear magnetic resonance (NMR), (i.e. the resonant absorption of radio frequency radiation by magnetic nuclei $\left.\left(\Delta M_{s}=0, \Delta m_{1}= \pm 1\right)\right)$ in a technique called 'electron nuclear double resonance' or ENDOR described next. In this technique, the magnetic moment of the proton in a beam of hydrogen atoms has been determined accurately (Winkler et al 1972).

\subsection{Electron nuclear double resonance}

Electron nuclear double resonance or ENDOR, is a technique in which the conditions for the realization of ESR and NMR are established simultaneously, as can be easily seen from figure 4 . To begin with the static magnetic field is set at one of the ESR transitions (say $H_{1}$ ) and the microwave power level increased until the transition is partially saturated. Then a radio frequency field at high power is applied and the frequency varied until it matched $h v_{1}$, at which frequency there will be a desaturation of the ESR line with the eventual reestablishment of the ESR signal. In other words, the NMR is detected through changes in the level of the ESR signal.

The so-called ENDOR transition is observed only when mutual spin flips occur, for which $\Delta\left(M_{s}+m_{1}\right)=0$. There are two such transitions in this case, at $H_{1}$ and $H_{2}$, which occur at nuclear frequencies $\nu_{1}$ and $\nu_{2}$, and with the selection rule $\Delta M_{\mathrm{s}}=0, \Delta m_{\mathrm{l}}= \pm 1$ (note that the 
ENDOR selection rule is the opposite of the ESR selection rule $\Delta M_{\mathrm{s}}= \pm 1, \Delta m_{\mathrm{t}}=0$ ).

$$
h v_{1}=-\frac{h a}{2}+g_{\mathrm{N}} \mu_{\mathrm{N}} H_{0} \text {, }
$$

or

$$
h v_{2}=\frac{h a}{2}+g_{\mathrm{N}} \mu_{\mathrm{N}} H_{0} .
$$

It is important to note from (20) that while the difference $v_{1}-v_{2}$ measures $a$ directly, the mean frequency $\left(v_{1}+v_{2}\right) /$ 2 measures $g_{\mathrm{N}}$ and unambiguously identifies the interacting nucleus. (See table 2 for $g_{\mathrm{N}}$ values of magnetic nuclei).

The principal advantage of the basic ENDOR technique that combines the resolution of ESR with the sensitivity of NMR is the precise determination of the spin Hamiltonian parameters, due to the greater rate of energy absorption at microwave frequency and the greater population differences involved in the ENDOR levels.

Furthermore, ENDOR spectra are simpler than the corresponding ESR spectra. If several nuclei with the same or similar interactions are coupled to the unpaired electron, the ESR pattern becomes complicated and the 'superhyperfine' structure is not usually resolved. But in ENDOR, all nuclei with the same interaction can give rise to only two $(S=1 / 2)$ ENDOR lines which greatly enhance the resolution.

In the semiconducting materials containing impurities, to be discussed later, as in X-irradiated alkali halides, and in alkaline-earth halides and oxides (Henderson and Garrison 1973; Spaeth and Koschnick 1991), the ENDOR spectra are markedly dependent on the orientation of the crystalline solid in the applied field, so that the Hamiltonian of (17) is now anisotropic. The point symmetry of the defect (e.g. tetrahedral for $\mathrm{Si}$ and octahedral for $\mathrm{MgO}$ at $\mathrm{Mg}^{++}$site) helps to determine at least one principal axis of the defect so that the latter can be identified. In diagonal form (17) is

$$
\hat{\mathscr{H}}=\mu_{\mathrm{B}} \Sigma g_{\mathrm{ii}} H_{\mathrm{i}} \hat{S}_{\mathrm{i}}-g_{\mathrm{N}} \mu_{\mathrm{N}} H \cdot \hat{l}+h \Sigma A_{\mathrm{ij}} \mathrm{Si} I_{\mathrm{i}}+h \Sigma Q_{\mathrm{ii}} \hat{l}_{\mathrm{i}} \hat{l}_{\mathrm{i}},
$$

where $\mathbf{H}, \hat{S}$ and $\hat{I}$ are written in the principal axis system of $g, A$, and $Q$ tensors.

Most generally the electron interacts with $n$-shells (constituted by first $(n=1)$, second $(n=2)$ etc nearest neighbours in the crystal structure) of equivalent nuclei (e.g. 3d-electron of $\mathrm{Cr}^{+}$in $\mathrm{Si}$ interacting with far-flung $\mathrm{Si}$ neighbours or electron trapped at $\mathrm{Cl}^{-}$vacancy in $\mathrm{X}$-irradiated $\mathrm{KCl}$ interacting with distant $\mathrm{Cl}$ neighbours), the number of equivalent nuclei, $k$ being different in each shell, so that the spin Hamiltonian becomes

$$
\begin{aligned}
\hat{\mathcal{H}}=\mu_{\mathrm{B}} \hat{S} \cdot \tilde{g} \cdot H & \\
& +\sum_{n}\left[-g_{\mathrm{N}} \mu_{\mathrm{N}} \tilde{H}+h \hat{l}_{\mathrm{n}} \cdot \tilde{A}_{\mathrm{n}} \hat{S}+h Q I_{\mathrm{n}}^{2}\right],
\end{aligned}
$$

which has eigenvalues

$$
\begin{aligned}
E= & M_{\mathrm{s}} g_{\mathrm{eff}} \mu_{\mathrm{B}} H+\sum_{n}\left\{-m_{\mathrm{I}}(n) g_{\mathrm{n}} \mu_{\mathrm{N}} H+h M_{\mathrm{s}} m_{\mathrm{I}}(n)\right. \\
& \times[A(n)+B(n)]\left\{3 \cos ^{2} \theta+\frac{1}{2} h P_{\mathrm{n}}\left[3 \cos ^{2} \theta_{\mathrm{n}}-1\right]\right. \\
& \left.\left.\times\left[m_{\mathrm{l}}^{2}(n)-\frac{I(I+1)}{3}\right]\right\}\right\},
\end{aligned}
$$

where $g_{\text {eff }}$ is the effective $g$ value, $A_{0}(n)$ and $B(n)$ are the isotropic and anisotropic hyperfine structure parameters, corresponding to the $n$th nucleus $\left(A_{0}=1 / 3\left(A_{\mathrm{xx}}+A_{\mathrm{yy}}+A_{\mathrm{zz}}\right)\right.$ and $\left.B=1 / 6\left(-A_{z z}-A_{x x}-A_{y y}\right)\right), \theta_{\mathrm{n}}$ is the angle made by the symmetry $(Z)$ axis of the paramagnetic complex (which is along the magnetic field) involving the $n$th nucleus, with the principal axis of the complex.

And the total nucleus spin quantum number $m_{\mathrm{I}}(n)$ of the $n$th shell is given by

$$
\begin{aligned}
m_{\mathrm{I}}(n) & =\sum_{k} m_{\mathrm{I}}(k) \\
& =k I, k I-1, \ldots-(k I-1), k \delta .
\end{aligned}
$$

The intensity of each hyperfine components depends on (i) the number of ways in which $m_{\mathrm{I}}(n)$ is compounded and (ii) the abundance of isotopic species (table 2).

The case of ENDOR of transition metal ion, such as interstitial $\mathrm{Cr}^{+}$in $\mathrm{Si}$ due to $\mathrm{Si}$ ligands, illustrates a system with $S>1 / 2$ and $I=1 / 2$. The effective spin Hamiltonian for this system with $S=1 / 2$ is

$$
\begin{aligned}
\hat{\mathcal{H}}=g \mu_{\mathrm{B}} H \cdot S+1 / 6 a\left[S_{x}^{4}+S_{y}^{4}-S_{z}^{4}-1 / 5 S(S+1)\right. \\
\left.\quad \times\left(3 S^{2}+3 S-1\right)\right]+\sum_{i}\left(S \cdot \tilde{A}_{\mathrm{i}} I_{\mathrm{i}}-g_{\mathrm{N}} \mu_{\mathrm{N}} H \cdot I_{\mathrm{i}}\right) .
\end{aligned}
$$

Here, the first term describes the impurity in electron-magnetic field interaction, the second term is the cubic field splitting i.e. the splitting of the ground state multiplet of $\mathrm{Cr}^{+}$by a crystalline electric field of cubic symmetry, the third term is the interaction between the impurity electron and a ${ }^{29} \mathrm{Si}$ nucleus, and the last term represents the nuclear Zeeman interaction.

The NMR transitions for this system occur at

$$
\nu=\nu_{\mathrm{z}}+M_{\mathrm{s}} A_{\mathrm{eif}} / h\left(M_{\mathrm{s}}=-5 / 2, \ldots+5 / 2\right),
$$

where $v_{\mathrm{z}}=-g_{\mathrm{N}} \mu_{\mathrm{N}} H / h$ is the nuclear Zeeman frequency and $A_{\text {eff }}$ the effective hyperfine interaction. The EPR transitions and the ENDOR transitions are compared in the schematic energy level diagram (figure 5). The experimental technique is briefly described in $\S 5$ and the applications are discussed in $\S 8$. 


\subsection{Electron spin echo technique}

Advanced materials are not often available in single crystal form but are readily obtainable as ceramics and glasses, and they often give broad EPR spectra (linewidth $\sim 1 \mathrm{mT}$, even at low temperature) due to strains and unresolved magnetic interactions due to nearby nuclei as seen by ENDOR in favourable cases. But in cases where this broadening is not resolved, one needs a technique to overcome this problem. This is possible through the electron spin echo technique that utilizes the programmed pulsed excitation of the sample through the inherently coherent, high power microwave radiation and detects the 'echo' signals that the sample 'emits' which may be observed in the time domain to obtain 'phase memory' or 'spin coherence times' of the spin system. Let us illustrate the principle of two-pulse spinecho formation (Slichter 1978). Let at time $t<0$ or $\mathrm{O}^{-}$ (figure 6a) the equilibrium magnetization $\left(M_{0}\right)$ of the spin system point along $\mathrm{z}$-axis of an orthogonal coordinate system. Then at $t=0$, upon application of a $\pi / 2$ or $90^{\circ}$ pulse of microwaves, $M_{0}$ would be tilted by $90^{\circ}$ away from its equilibrium position (figure $6 \mathrm{~b}$ ) parallel to the microwave magnetic field $H_{1}$ along $\mathrm{x}$-axis in time $t_{\mathrm{w}}=\pi / 2|\gamma| H_{1}$, where $\gamma$ is the gyromagnetic ratio. Left to itself, the pulse will decay, usually exponentially, producing transient or free induction decay of the mag-

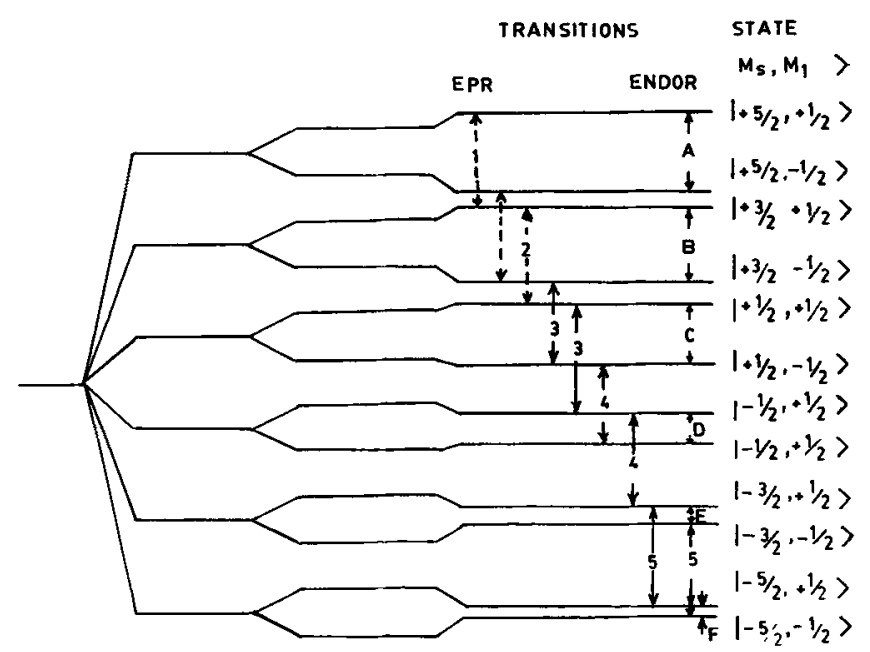

$\begin{array}{lllll}\text { UNSPLIT } & \text { ZERO } & \text { ELECTRON } & \text { NUCLE } & \text { HYPERFINE } \\ \text { GROUND } & \text { FIELD } & \text { ZEEMAN } & \text { ZEEMAN } & \text { SPLITTING } \\ \text { STATE } & \text { SPLITTING } & \text { MS } 9 \mu_{B} H & -m_{2} g_{N} \mu_{N} H & M_{S} M_{I} \text { AEI }\end{array}$

Figure 5. EPR $\left(\Delta M_{\mathrm{s}}= \pm 1, \Delta m_{1}=0\right)$ and ENDOR $\left(\Delta M_{\mathrm{s}}=0\right.$, $\Delta m_{\mathrm{I}}= \pm 1$ ) transitions in monovalent chromium. The states involved in the transitions are labelled as also the transitions (Kemp et al 1987). netization (figure 6c). This is due to slightly different precession frequencies of inhomogeneous groups of electrons in the sample. A pulse of length $\tau=2 t_{\mathrm{w}}$ or $\pi$-pulse applied at $t=\tau$ reverses the direction of the electron magnetization vector. Note that these situations are not equilibrium ones and therefore the system will return to equilibrium at a definite rate, which can be used to determine the relaxation times of the system. The echo is formed $2 \tau$ seconds later (figure 6e), due to a refocusing of all the elements of magnetization and reconstruction of the original magnetization.

The so-called phase memory time $T_{2}^{*}$ governs the decay of transwerse magnetization (i.e. $M$ in the $x-y$ plane) created by the $\pi / 2$ pulse, while the spin-lattice relaxation time $\left(T_{1}\right)$ governs the decay of the longitudinal magnetization (i.e. $M$ along $\mathrm{z}$-axis) created by the $\pi$-pulse. In fact, $T_{1}$ is a measure of the time taken for the spin magnetization energy to be transferred to the thermodynamic levels of the lattice, and is of order $10^{-6} \mathrm{sec}$ or less for electrons.

In a given material containing unpaired spins the dependence of the echo signal amplitude on the time interval $\tau$, which is usually recorded in spin echo experiments, is governed by: (i) the existence of magnetic relaxation process $\left(T_{1}\right.$ and $\left.T_{2}^{*}\right)$ that return the spin system perturbed by microwave pulse to the equilibrium state, and (ii) the frequently observed interaction of the unpaired electrons with magnetic nuclei causing oscillations in the echo signal decay or 'electron spin echo envelope modulation'.

These two processes are statistically independent and thus one observes a product of monotonously decaying and oscillating functions that give information about nuclear environment around the unpaired electron (Dikanev and Ashtashkin 1989; Mims 1989).

\section{The ESR spectrometer}

\subsection{The continuous wave ESR spectrometer}

The continuous wave (CW) ESR experiment, as distinguished from the pulsed ESR experiment, to be discussed later, is a steady-state experiment, in which the irradiation of the unpaired electron spin system with microwave keeps the system in equilibrium. The CW ESR spectrometer (Wilmshurst 1967; Alger 1968; Poole 1983) uses an uninterrupted, monochromatic 'beam' of microwaves of appropriate frequency (e.g. $X$ band $\sim 9 \mathrm{GHz}$ from a Klystron or Gunn Oscillator), a suitable microwave guiding system, a cylindrical or rectangular metallic cavity to house the sample and a detecting system to monitor the spectrum and finally an electronic system to record the ESR spectrum. The Klystron frequency may be locked to the cavity frequency through auxiliary 
circuitry and measured by comparison with a quartz crystal standard in the frequency counter. A linearly varied static magnetic field derived from an electromagnet with the pole pieces symmetrically flanking the cavity, or, a superconducting magnet separates the Kramers' doublets (i.e. two energy levels that differ only in spin orientation) and prepares the sample for the resonance experiment. The typical spectrometer for X-band ESR work is shown in schematic in figure 7. The typical cavities used and the magnetic field patterns inside them are also shown (figure 8). These cavities which may be tuned to the incoming microwaves concentrates the radiation in its standing wave pattern. The sample is placed in the region corresponding to the maximum microwave magnetic field. The level of power in the cavity may be ascertained by tapping a fraction of it to a crystal (Si-rectifier) detector which converts the microwave radiation into a dc signal.

A small alternating magnetic field modulation (figure 9a) super-imposed on the static field, enables the field to be swept through resonance twice during each cycle. The output signal from crystal detector will be modulated at twice the frequency of modulation. For high sensitivity the modulation frequency should be high compared to audio frequency. Of course there is a trade off between this frequency and the excess noise produced by the detecting system. There is a broad minimum around $30 \mathrm{MHz}$, which corresponds to the maximum sensitivity, but the excess crystal noise ( $1 / f$ noise) at $100 \mathrm{KHz}$ is very small. This fact coupled with the simplicity of design has made $100 \mathrm{KHz}$ as the standard modulation frequency of most commercial spectrometers. Usually a pair of modulation coils are embedded in the wall of the cavity whose walls are thin enough to let the $100 \mathrm{KHz}$ modulation without attenuation but thick enough to be short for microwave frequencies. The static magnetic field strength required depends on the microwave band employed. X-band $(3.2 \mathrm{~cm})$ work requires up to $0.33 \mathrm{~T}$, while for Q-band $(\sim 8 \mathrm{~mm})$ work fields $\sim 1.3 \mathrm{~T}$ are needed. Work at shorter wavelengths (higher frequencies) such as $4 \mathrm{~mm}$ and $2 \mathrm{~mm}$ requires superconducting magnets which produce fields $\sim 5 \mathrm{~T}$. The magnetic field must be uniform over the volume of the specimen, to the extent of $0.1 \mathrm{ppm}$ to resolve spectra to better than $25 \mathrm{mG}$ (or $2.5 \mu \mathrm{T}$ ). Therefore it has to be stabilized and controlled by a Hall-effect probe located in the magnetic gap. A nuclear magnetic resonance fluxmeter (Gaussmeter) is employed to measure the magnetic field and may also be used for stabilization. Resonance field positions are usually accurate to 5 significant figures.

The usual method of detection is based on the 'super heterodyne' principle of mixing the signal reflected from cavity with the output of a local oscillator (Klystron operating at $39 \mathrm{MHz}$ above or below signal Klystron) to produce an intermediate frequency, which in turn is amplified and detected. (Note that $30 \mathrm{MHz}$ as mentioned before corresponds to maximum sensitivity). After detection, $100 \mathrm{KHz}$ signal is sent to a narrow band

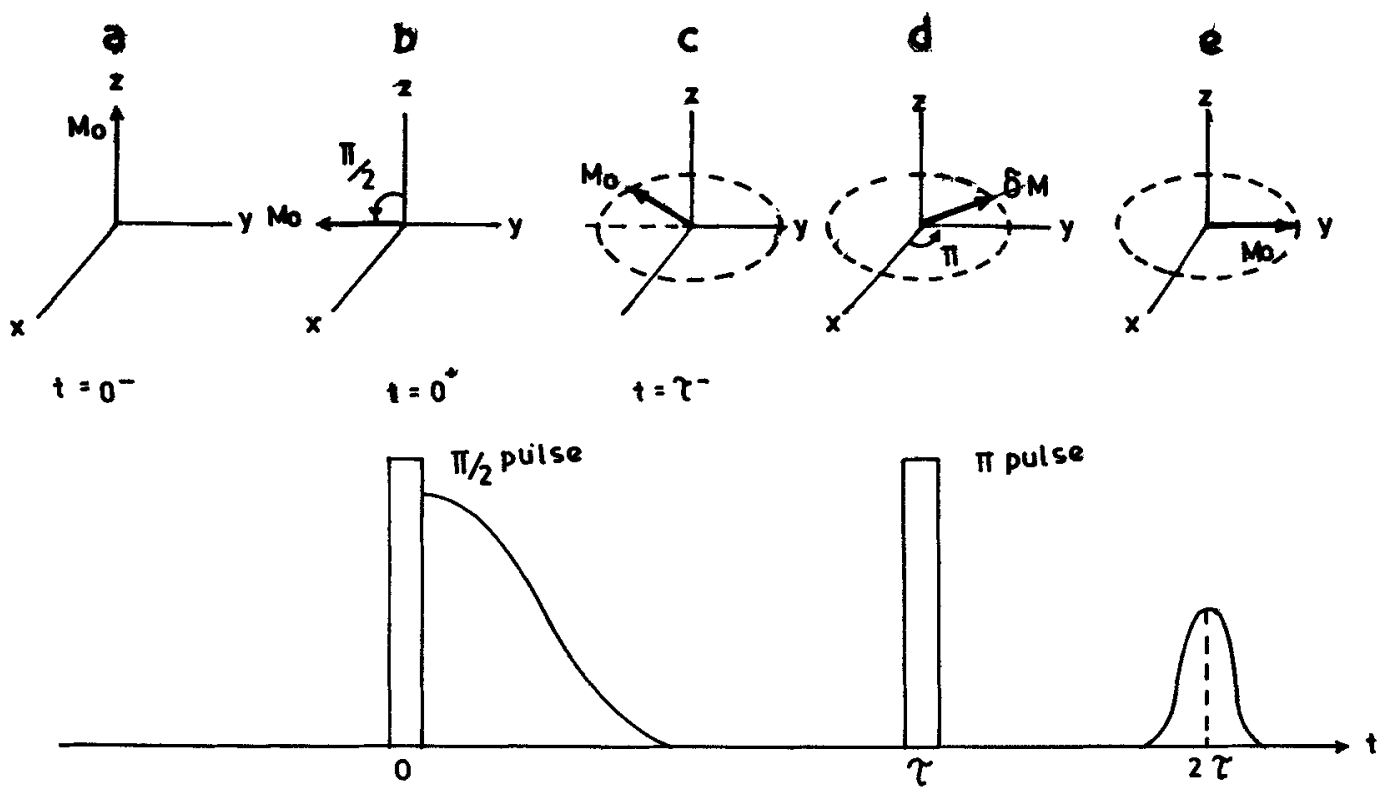

Figure 6. Schematic of the two-pulse electron-spin echo technique. The top half shows the positions of the equilibrium magnetization vector $\left(M_{0}\right)$ (a) before application of $\pi / 2$ pulse, with $M_{0}$ along $z$, (b) just on application of $\pi / 2$ pulse, $M_{0}$ along $y$, (c) the defocussing of $M_{0}$ in $x-y$ plane, (d) the refocussing of $M_{0}$ upon application of $\pi$-pulse, and, (e) the echo formation. The bottom half illustrates the pulse shape, decay and echo. 
amplifier and then on to a phase sensitive detector. The operation of the latter is as follows: The amplified signal is mixed with the output of the modulating $100 \mathrm{KHz}$ oscillator. If the two signals are in phase opposition, the output of the system is minimum, if the two phases coincide exactly, the output is maximum. The composite signal is rectified, filtered and recorded. The output signal, i.e. the final ESR spectrum naturally depends markedly on the relative phase and amplitude of the signal and reference voltages. The signal shapes before and after phase sensitive detection are shown in figure $9 \mathrm{~b}$.

The actual signal from the spectrometer is proportional to the first derivative of the absorption line contour. If signal dispersion is to be avoided, the amplitude of modulation sweep must be less than half the linewidth of the spectral line (figure 9a). The sensitivity of detection and display system depends only on the bandwidth of the actual recording equipment.

The voltage output of the phase-sensitive detector may be changed to a pulse train by a voltage-to-frequency counter and these pulses are counted and stored in the channels of the signal averager, which enables repetitive scans to be made through the spectrum to improve signal to noise ratio. Finally the output may be plotted as a curve or digitized using an analog-to-digital converter and computer-interfacing electronics.

\subsection{The pulsed ESR spectrometer}

The pulsed or the Fourier transform ESR spectrometer system (figure 10) detects the resonance in the time domain by observing the recovery of the magnetization of the spin system from a condition of extreme nonequilibrium to which it was deliberately driven previously.

In a simplified version of a pulsed-ESR spectrometer, the microwave radiation, from a high power $(\sim 1-2 \mathrm{~kW})$

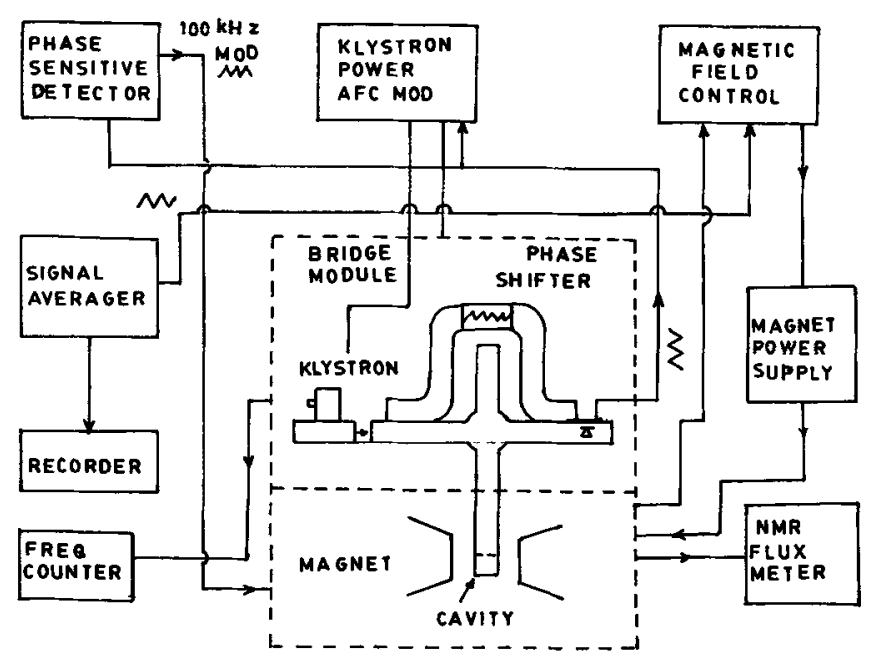

Figure 7. Block-diagram of a CW ESR spectrometer.
Klystron is converted into microwave pulses and shaped by a pulse gate. These pulses are then amplified by the travelling wave tube (TWT) amplifier unit and sent to the resonant cavity situated between the pole pieces of the electromagnet. The in-phase and out-of-phase components of the magnetization recovery signal are obtained simultaneously with separate detection channels. Finally these two signals are combined and recorded/displayed as the Fourier transform output.

A general time-domain spectrometer (Das et al 1986) used for relaxation experiments where (i) spin-lattice relaxation times could be measured in the pulsed mode, or (ii) time-dependence of ESR signal upon pulsed laser excitation of the sample could be monitored, is shown as a block diagram (figure 11). The computer selects the type of pulse sequence necessary for the instrument. The pulse programmer generates such a pulse sequence. The computer also controls the digitizer which accepts, after amplification the ESR signal from the microwave bridge, and starts digitizing the signal at the falling edge of the excitation pulse. After recording the signal, the digitizer transfers the digitized signal to the computer which in turn process it and outputs it suitably. The computer also controls the magnetic field. It can either scan the magnetic field (for $\mathrm{CW}$ operation) or hold it at a desired value (for time-domain operation). The field sensor gives the magnitude of the magnetic field. The main modules of this spectrometer are: (i) the Klystronbased microwave set up, (ii) the automatic frequency control (AFC) of Klystron-based on intermediate frequency oscillator, and (iii) the automatic phase control based on an electronic phase shifter - to maintain the correct phase relationship between the AFC signal and ESR signal, for any length of time.
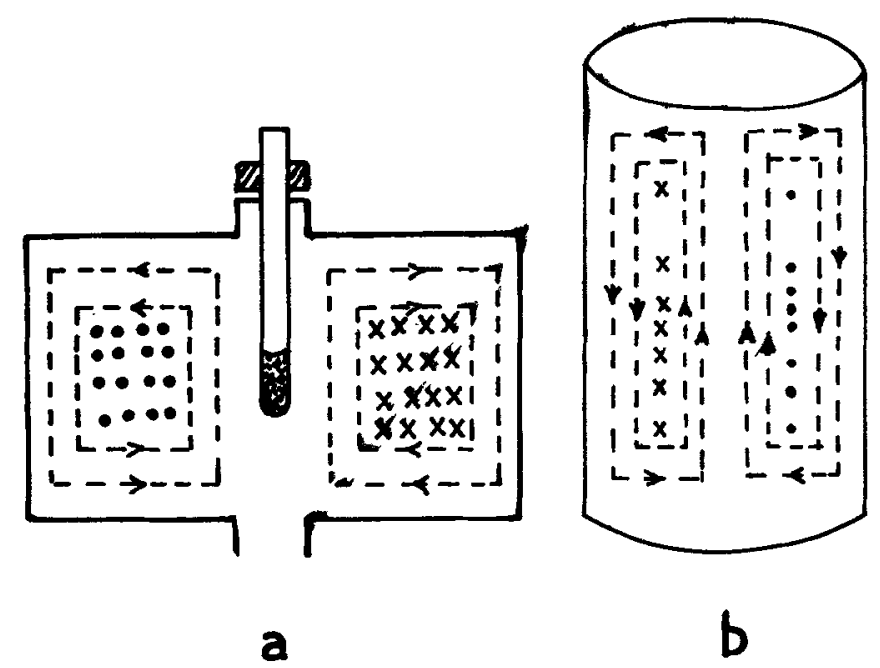

Figere 8. Cross-sectional view of (a) rectangular $\mathrm{TE}_{(102}$ and (b) cylindrical $\mathrm{TE}_{011}$ cavities showing microwave magnetic field as loops. In (a) the sample in a tube is positioned to be in a region of maximum field intensity. Dots and crosses indicate electric field or current distributions (Poole 1983). 
A number of special purpose continuous wave and pulsed ESR spectrometers have been built. Salient features of a few of these are summarized in table 3.

\subsection{ENDOR spectrometer}

The ENDOR spectrometer monitors and detects changes in the electron spin populations in a sample subjected to a simultaneous irradiation at microwave and nuclear magnetic resonance frequencies, at a dc magnetic field corresponding to an ESR transition which is partially saturated. This is done by choosing the microwave power high enough such that the transition probability for the microwave transition far exceeds the spin lattice relaxation rate, when the spin levels connected by the microwave transition become equally populated. This results in a decrease and an eventual disappearance of the observed ESR absorption because microwave emission and absorption probabilities are equal. But the levels not connected by the microwave transitions are not affected. Thus an ENDOR spectrum would be a record of the changes in the ESR signal as the NMR frequency is swept.

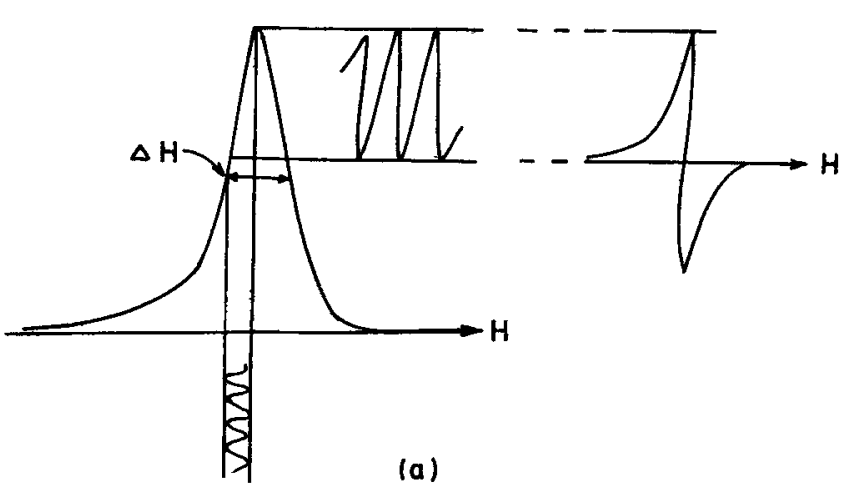

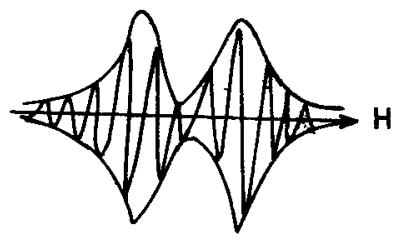

(i) (b)

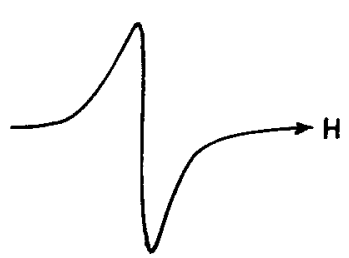

(ii)
Figure 9. Illustration of the principles of (a) modulation and (b) phase sensitive detection. The modulation signal amplitude should be less than half the linewidth $(\Delta H)$ of the ESR profile in order to get distortion-free signal. The detector produces the first derivative absorption signal when the magnetic field is swept.
The major problem in the ENDOR spectrometer is to get sufficient r.f. power to the sample. There are a few solutions to this problem: (i) placing an r.f. coil outside the cavity, either split the cavity to allow r.f. field inside (Holton and Blum 1962) or use a silvered glass cavity. This method preserves the $Q$ of the cavity (i.e. sensitivity) but is extravagant in the use of r.f. power, and (ii) the r.f. coil is placed inside the cavity so that less r.f. power is required. In any case the r.f. coil is driven by a power amplifier fed by an external tunable oscillator. The details of the ENDOR experiment are as follows: The ESR superheterodyne spectrometer (figure 7) is tuned for the observation of dispersion or absorption signal and the magnetic field is set to the centre of the line. This line is partially power saturated, while ensuring that the magnetic field stays on resonance. Then using the r.f. oscillator the nuclear transition is observed as a return of the unsaturated ESR signal. This simple procedure gives rise to baseline problems because of detection of a small change due to ENDOR in a large

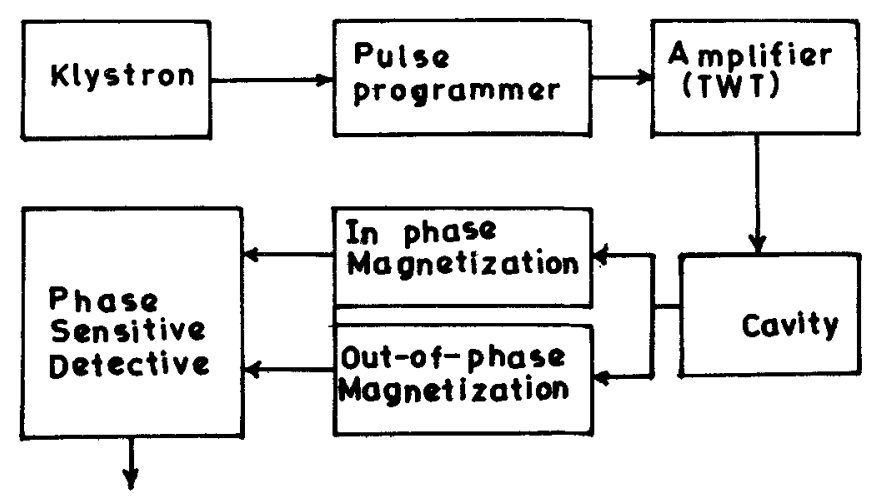

\section{F.T. Output}

Figure 10. Block diagram of a pulsed ESR spectrometer.

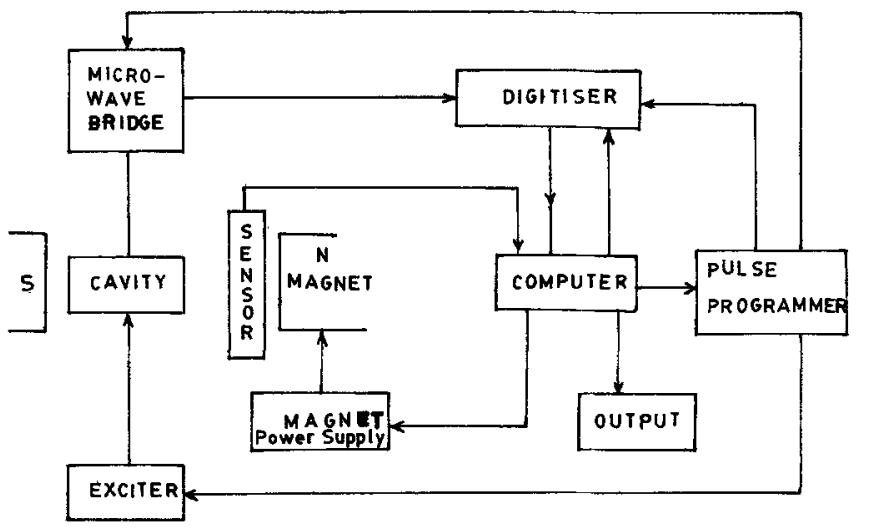

Figure 11. Block diagram of a general time-domain spectrometer (Das et al 1986). 
ESR absorption signal. The use of dispersion mode of recording also leads to electronic noise and baseline problems. However, using absorption mode with the r.f. power amplitude modulated at an audio frequency followed by lock-in detection of the ENDOR signal overcomes these problems. In the lock-in detection technique the signal input does not vary in phase relative to the reference signal. Thus the detector output is proportional to the absorption. An output proportional to the first derivative of the absorption can be obtained by frequency modulation of r.f. power at an audio frequency $\nu_{\mathrm{m}}$ and using a phase-sensitive detector with reference signal set at $v_{\mathrm{m}}$. This method (Doyle 1962) is convenient for investigating hyperfine structure of defects in materials. Signal averaging increases the sensitivity of the ENDOR spectrometer (Reichart and Pereli 1966). Commercial spectrometers are now available for ENDOR work (Bruker Almanac 1993).

Table 3. ESR spectrometers.

\begin{tabular}{|c|c|c|c|c|c|}
\hline & Type & Frequency & Main components and characteristics & Used for & Ref \\
\hline 1. & $\begin{array}{l}\text { Continuous wave } \\
(\mathrm{CW}) \text {, portable }\end{array}$ & $9.1-9.6 \mathrm{GHz}$ & $\begin{array}{l}\text { Nd-Fe-B permanent magnet }(1.3 \mathrm{~T}) 275 \mathrm{~Hz} \\
\text { modulation } \\
\text { Volume: } 0.33 \times 0.12 \times 0.25 \mathrm{~m}^{3}\end{array}$ & $\begin{array}{l}\text { Radiation dosimetry, dating } \\
\text { and ESR microscopy }\end{array}$ & a \\
\hline 2. & $\mathrm{CW}$ & $300 \mathrm{MHz}$ & $\begin{array}{l}\text { Loop gap resonator: } 10.2 \mathrm{~cm} \text { outer diameter, } \\
15.0 \mathrm{~cm} \text { long with } 1.55 \mathrm{~cm} \text { gap separation } \\
\text { Unloaded Q: } 5000 \text {. Automatic tuning, coupling } \\
\text { and phase control circuits. Magnetic field } \\
\text { gradient coils }\end{array}$ & $\begin{array}{l}\text { In vivo imaging of large } \\
\text { biological samples }\end{array}$ & $\mathrm{b}$ \\
\hline & $\mathrm{CW}$ & $250 \mathrm{MHz}$ & Strip-line type resonator & In vivo imaging & c \\
\hline & $\begin{array}{l}\text { CW microstrip } \\
\text { induction } \\
\text { spectrometer }\end{array}$ & X-band & $\begin{array}{l}\text { Microstrip bimodal square patch resonator } \\
\text { Superheterodyne detection }\end{array}$ & $\begin{array}{l}\text { Thin film and interfacial } \\
\text { ESR }\end{array}$ & d \\
\hline 5. & Pulsed & $X$-band & $\begin{array}{l}\text { Klystron, Waveguide-based diode switches, } \\
\text { Boxcar integrator }\end{array}$ & $\begin{array}{l}\text { Measurement of spin- } \\
\text { lattice relaxation and spin- } \\
\text { echo lifetime }\end{array}$ & e \\
\hline 6. & CW/pulsed & X-band & $\begin{array}{l}\text { Computer-controlled magnetic field-microwave } \\
\text { frequency interlock saturation recovery }\end{array}$ & $\begin{array}{l}\text { Spin relaxation in stable } \\
\text { radicals, laser photolysis } \\
\text { with transient radicals }\end{array}$ & f \\
\hline 7. & $\mathrm{CW} /$ pulsed & & $\begin{array}{l}\text { High power }(1 \mathrm{~kW}) \text { TWT amplifier. Low power } \\
(20 \mathrm{w}) \mathrm{CW} \text { TWT amplifier }\end{array}$ & & g \\
\hline & Spin-echo & & $\begin{array}{l}\text { Pulsed Travelling Wave Tube (TWT) 'pulse } \\
\text { former'. Low noice TWT-based homodyne } \\
\text { detection }\end{array}$ & & h \\
\hline 9. & $\begin{array}{l}\text { Pulsed/CW } \\
\text { (Transmission) }\end{array}$ & $140 \mathrm{GHz}$ & $\begin{array}{l}\text { Pulsed: Extended interaction Klystron }(300 \mathrm{w}) \text {. } \\
3.0 \mathrm{~T} \text { field; Fabry-Perot resonator; signal } \\
\text { averaging CW: phase locked } 139.5 \mathrm{GHz} \text { Gunn } \\
\text { oscillator; } 500 \mathrm{~Hz} \text { modulation and phase } \\
\text { detection; digital oscilloscope }\end{array}$ & $\begin{array}{l}\text { Dynamic parameters of } \\
\text { electrons spin systems with } \\
\text { high sensitivity, shorter } \\
\text { time scales and improved } \\
\text { spectral resolution }\end{array}$ & $\mathrm{i}$ \\
\hline & $\begin{array}{l}\text { Magnetic resonance } \\
\text { force microscopy }\end{array}$ & & $\begin{array}{l}\text { Detection of ESR through periodic saturation } \\
\text { of electron spin magnetization; Force between } \\
\text { field gradient and spin moments measured with } \\
\text { a microcantilever }\end{array}$ & Imaging & j \\
\hline
\end{tabular}

aNakanishi A, Sugahara N and Furuse A 1993 Appl. Radiat. Isotopes 44357

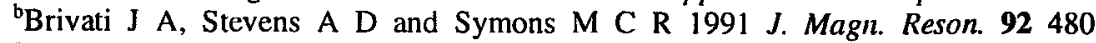

'Halpern H J et al 1989 Rev. Sci. Instrum. 601040

${ }^{d W a l l a c e}$ W J and Silsbee R H 1991 Rev. Sci. Instrum. 621754

${ }^{\text {e} B r o w n ~ I ~ M ~ a n d ~ S l o o p ~ D ~ J ~} 1970$ Rev. Sci. Instrum. 411774

${ }^{\dagger}$ Das et al 1987

${ }^{g}$ Quin R W, Eaton G R and Eaton S S 1987 Rev. Sci. Instrum. 581709

hBlumberg W E, Mims W B and Zuckermann D 1973 Rev. Sci. Instrum. 44546

'Prisner T F, Un S and Griftin R G 1992 Israel J. Chem. 32357

'Zhang Z et al 1996 J. Appl. Phys. 806930 


\section{The ESR spectrum and its analysis}

\subsection{The ESR spectrum}

The ESR spectrum is generally a plot of the first derivative of the imaginary part of the complex magnetic susceptibility of the paramagnetic system with respect to the applied dc magnetic field. The spectrum may consist of a single line or a group of lines, and could vary with changes in temperature or the orientation of the specimen (e.g. a stretched polymer or a Si single crystal) relative to the magnetic field. Four basic quantities derivable from the spectrum are: (i) $g$-factor or $g$-tensor, (ii) hyperfine splitting parameter or A-tensor, (iii) line shape and line width, and (iv) double integrated intensity.

An analysis of these four quantities reveal the following information about the system: (i) the nature of the paramagnetic centre or complex, i.e. electron or 'hole'-type, (ii) the precise identity of the complex/centre in terms of its nearest neighbours and symmetry, (iii) the distribution of unpaired spin density over the neighbours, and (iv) the number of paramagnetic centres per unit volume, and thus the bulk paramagnetic susceptibility of the sample.

6.1 a The g-factor: In the simplest of cases - that of a free spin system $S=1 / 2$ with a single line spectrum due to the transition from $M_{\mathrm{s}}=-1 / 2$ to $M_{\mathrm{s}}=+1 / 2-$ the resonance condition $h \nu=g \mu_{\mathrm{B}} H_{\mathrm{r}}$, where $h$ is the Planck's constant and $\mu_{\mathrm{B}}$ the Bohr magneton gives the $g$-factor, the frequency $v$ is accurately known and $H_{\mathrm{r}}$ is measured accurately from the spectrum. Thus: $g=21.4198 \bar{v} / H_{\mathrm{r}}\left(\bar{\nu}\right.$ in $\mathrm{cm}^{-1}, H_{\mathrm{r}}$ in deca Tesla or $g=0.71557 \times 10^{-6}\left(v\right.$ in $\mathrm{Hz}, H_{\mathrm{r}}$ in $\left.\left.\mathrm{mT}\right)\right)$. However, it is possible to determine $g$ accurately using standard samples along with the test samples. A selection of these calibration samples are given in table 4 .

In general, neither is $g=2.0023$, the free electron value, nor is it a single number, i.e. isotropic with respect to sample rotation within the magnetic field. The anisotropic $g$ (particularly in materials with transition metal ions as impurities) arises from the coupling of the orbital angular momentum to the spin angular momentum via spin-orbit interaction. The electron Zeeman interaction responsible for the ESR spectrum is represented as the leading term in the spin Hamiltonian.

$$
\mathcal{H}_{\mathrm{z}}=\mu_{\mathrm{B}} \hat{S} \tilde{g} \hat{H},
$$

where $\tilde{g}$ is a second-rank symmetric tensor and $\hat{S}$ the effective spin operator. With reference to a Cartesian coordinate frame $x, y, z$ in which the electron magnetization is parallel to the applied magnetic field when the latter has components along $\mathrm{x}, \mathrm{y}$ and $\mathrm{z}, \mathscr{H}_{\mathrm{z}}$ becomes

$$
\mathscr{H}_{\mathrm{z}}=\mu_{\mathrm{B}}\left(g_{\mathrm{x} x} S_{\mathrm{x}} H_{\mathrm{x}}+g_{\mathrm{yy}} S_{\mathrm{y}} H_{\mathrm{y}}+g_{\mathrm{zz}} S_{\mathrm{z}} H_{\mathrm{z}}\right) \text {. }
$$

A study of a monocrystalline material (e.g. $\mathrm{BaTiO}_{3}: \mathrm{Co}$ ) or a glassy material (e.g. photochromic glass) is aimed at obtaining $g_{\mathrm{xx}}, g_{\mathrm{yy}}$ and $g_{\mathrm{zz}}$ and their direction cosines with respect to $\mathrm{x}, \mathrm{y}$ and $\mathrm{z}$ through angular variation of ESR spectrum or computer simulation of observed spectrum - a first step in the analysis of the spectrum in which further details are available. In general, a centre with $g_{\mathrm{av}}=\left(g_{\mathrm{xx}}+g_{\mathrm{yy}}+g_{\mathrm{zz}}\right) / 3>2.0036$, is referred to as a 'hole centre' (e.g. V centre in X-irradiated alkali halides) while a centre with $g_{a v} \leq 2.0036$ is called an 'electronexcess centre' (F-centre in alkali halides) (Henderson and Garrison 1973).

In case of paramagnetic systems, in which the ground and excited states are mixed by spin $(S)$-orbit $(L)$ interaction $(\lambda L \cdot S)$, the elements of the $g$-tensor are given by

$$
g_{\mathrm{ij}}=2 \delta_{\mathrm{ij}}-2 \lambda \frac{\left\langle n\left|L_{\mathrm{i}}\right| 0\right\rangle\left\langle 0\left|L_{\mathrm{j}}\right| n\right\rangle}{E_{\mathrm{n}}-E_{0}},
$$

where $\delta_{i j}=|i| i=j$ or 0 otherwise, $|0\rangle$ is the orbital singlet ground state of energy $E_{0} .|n\rangle$ is the excited state

\begin{tabular}{|c|c|c|c|}
\hline Standard & $g$-value & $\begin{array}{l}\Delta H_{\mathrm{pp}} \\
(\mathrm{mT})\end{array}$ & $\begin{array}{l}\text { Spin concentration } \\
\left(\mathrm{cm}^{-3}\right)\end{array}$ \\
\hline $\begin{array}{l}\text { DPPH (Di phenyl picryl hydrazyl } \\
\text { (freshly crystallized)) }\end{array}$ & $2 \cdot 0037(2)$ & $0 \cdot 28$ & $1.5 \times 10^{2}$ \\
\hline $\mathrm{CuSO}_{4} \cdot 5 \mathrm{H}_{2} \mathrm{O}$ & $\begin{array}{l}g_{\|}=2.2700 \\
g_{\perp}=2.0800 \\
\langle g\rangle=2.1433\end{array}$ & - & - \\
\hline Si : P $4 \cdot 3 \mathrm{~K}$ & $1.99869(2)$ & - & {$[\mathrm{P}]=1.7 \times 10^{18}$} \\
\hline $\begin{array}{l}\text { Charred dextrose powder } \\
\text { Powdered coal diluted with } \mathrm{KCl} \text { (pitch) }\end{array}$ & $2 \cdot 0023(3)$ & 0.06 & $5 \times 10^{20}$ \\
\hline (a) weak pitch & $2 \cdot 0028$ & $0 \cdot 17$ & $1 \times 10^{13}$ \\
\hline (b) strong pitch & 2.0028 & $0 \cdot 17$ & $3 \times 10^{15}$ \\
\hline
\end{tabular}
of energy $E_{\mathrm{n}}$ and $\lambda$ is the spin-orbit coupling constant

Table 4. Standards for calibration of $g$-values. 
(see table 2 for numerical values of $\lambda$ ). The larger the $\lambda$, the larger is the deviation of $g$ from 2.0023 , the 'free spin' value.

6.1b The hyperfine coupling constant (A): Just as the quantization of electron spin produces the simple ESR spectrum, quantization of the nuclear spin $\left(I,(2 I+1) m_{1}\right)$ of the magnetic nucleus associated with the unpaired electron (e.g. $\mathrm{H}$ atom, $S=1 / 2, I=1 / 2$ ) gives rise to additional splitting of levels $\left(\Delta M_{\mathrm{s}}= \pm 1, \Delta m_{\mathrm{I}}=0\right)$ due to the interaction between the electronic magnetic dipole and nuclear magnetic dipole.

In terms of the spin operators $S$ and $I$,

$$
\mathcal{H}_{\mathrm{hf}}=S \cdot A \cdot I,
$$

where $A$ is a symmetric second-rank tensor like $g$. In a reference frame xyz where $A$ is diagonal,

$$
\mathcal{H}_{\mathrm{hf}}=A_{\mathrm{xx}} S_{\mathrm{x}} I_{\mathrm{x}}+A_{\mathrm{yy}} S_{\mathrm{y}} I_{\mathrm{y}}+A_{\mathrm{zz}} S_{\mathrm{z}} I_{\mathrm{z}} \text {. }
$$

In a majority of cases, however, the A-tensor is axially symmetric, so that

$$
\mathcal{H}_{\mathrm{hf}}(\text { axial })=A_{1} S_{\mathrm{z}} I_{\mathrm{z}}+A_{1}\left(S_{\mathrm{x}} I_{\mathrm{x}}+S_{\mathrm{y}} I_{\mathrm{y}}\right)
$$

where $\mathrm{z}$-axis being the axis of symmetry of the complex containing the unpaired electron. The A-tensor contains information about (i) isotropic contribution $\left(A_{\text {iso }}\right)$, due to s-electron density at the magnetic nucleus and (ii) the anisotropic contribution due to the spin and orbital dipole-dipole interactions with the nuclei. The socalled Fermi-constant interaction is the origin of $A_{\text {iso }}=\left(A_{\|}+2 A_{1}\right) / 3$. This interaction is given by

$$
\mathcal{H}_{\text {contact }}=\frac{8 \pi}{3} g \cdot \mu_{\mathrm{B}} g_{\mathrm{N}} \mu_{\mathrm{N}}|\psi(0)|^{2} \quad \text { S.I., }
$$

from which

$$
\begin{aligned}
A_{\mathrm{iso}} & =\left(\frac{8 \pi}{3}\right) g \mu_{\mathrm{B}} g_{\mathrm{N}} \mu_{\mathrm{N}}|\psi(0)|^{2} \\
& =4.24 g_{\mathrm{N}}|\psi(0)|^{2}(\mathrm{mT}) \text { using } \mu_{\mathrm{N}}=\mu_{\beta} / 1836,
\end{aligned}
$$

where $g$ and $g_{\mathrm{N}}$ are electron and nuclear $g$ factors and $\mu_{\mathrm{B}}$ and $\mu_{\mathrm{N}}$ are Bohr and nuclear magnetons, and $|\psi(0)|^{2}$ (in $\mathrm{cm}^{-3}$ ) is the unpaired electron density at the nucleus. A comparison of the experimental value of $|\psi(0)|^{2}$ (table 2) with the theoretical value for complete localization yields the amount of s-character in the unpaired electron wave function. For a wave function

$$
\begin{aligned}
& \psi=a|s\rangle+\sqrt{\left(1-a^{2}\right)}|p\rangle, \\
& \frac{A_{\text {iso }}(\text { expt. })}{A_{\text {iso }} \text { (theory) }}=a^{2} .
\end{aligned}
$$

$A_{\text {iso }}$ (theory) has been computed for all the magnetic atoms from $\mathrm{H}$ to $\mathrm{Bi}$ (McMillan and Halpern 1971). In the case of paramagnetic ions such as $\mathrm{Cu}^{++}\left(3 d^{9}\right)$ which give a large value of $A_{\text {iso, }}$, despite apparently small spin density at the nucleus, Fermi contact interaction cannot account for $A_{\text {iso }}$. In such cases, the inner (core) $s$ electrons (e.g. $3 s$ in the case of $\mathrm{Cu}$ ) are polarized by the valence electrons, and these 'core-polarized' electrons have a sizeable spin density at the ${ }^{65.63} \mathrm{Cu}$ nucleus to give a large $A_{\text {iso }}$ given by:

$$
\begin{aligned}
& A_{\text {iso }} \text { (core polarization) }= \\
& \quad \frac{8 \pi}{3} g_{\rho} \mu_{\rho} g_{1} \mu_{1} \sum_{n s}\left\{\left.\left|\psi_{n s}(0)\right| \uparrow\right|^{2}-\left.\left|\psi_{n s}(0)\right| \downarrow\right|^{2}\right\},
\end{aligned}
$$

where arrow ' $\uparrow$ ' indicates the spin state of the polarizing electron (McMillan 1974).

The anisotropic part of the hyperfine interaction, $A_{\text {aniso }}$ is due to the dipole-dipole interaction between electron and nuclear dipoles.

$$
\mathscr{H}_{\text {dipolar }}=g_{\mathrm{e}} g_{\mathrm{N}} \mu_{\mathrm{B}} \mu_{\mathrm{N}}\left[\frac{S \cdot I}{r^{3}}-\frac{3(S \cdot r)(I \cdot r)}{r^{5}}\right],
$$

which may be written in terms of the angle $\theta$ between the vector $(r)$ joining electron and nucleus and the magnetic field

$$
\mathcal{H}_{\text {dipolar }}=g_{\mathrm{e}} g_{\mathrm{N}} \mu_{\mathrm{B}} \mu_{\mathrm{N}}\left(\frac{3 \cos ^{2} \theta-1}{r^{3}}\right) I_{\mathrm{z}} S_{\mathrm{z}} .
$$

In terms of equivalent magnetic field, this dipolar interaction gives rise to the anisotropic part of hyperfine interaction

$$
A_{\text {aniso }}=\mu_{\mathrm{N}} g_{\mathrm{N}}\left\langle\frac{3 \cos ^{2} \theta-1}{r^{3}}\right\rangle I_{z}
$$

spatially averaged over all of the unpaired electron wave function. $A_{\text {aniso }}$ is thus proportional to $\left\langle 1 / r^{3}\right\rangle$ (table 2 ).

A typical polycrystalline ESR spectrum illustrating the anisotropic $g$ - and hyperfine structure, for the case of $\mathrm{Cu}^{++}$in $\mathrm{KHSO}_{4}$ (Ramasastry and Sunandana 1976) in an environment of rhombic symmetry is shown in figure 12.

6.1c Lineshape and linewidth: The shape of an ESR absorption or first derivative signal depends on the nature of the unpaired electron (ex: whether the electron is in a metal or a semiconductor or an insulator) and its immediate neighbourhood with which it interacts electro-statically and electro-magnetically. Indeed, the strength and nature of these interactions also give the ESR signal a nonzero linewidth. The uncertainty relation $\Delta E \cdot \Delta t \geq \hbar$ is an important basis for line broadening 
particularly for transition metal ion ESR. If $\Delta t=T_{1}$, the spin lattice relaxation time, then the magnitude of $T_{1}$ would decide the 'lifetime' broadening and linewidth. The linewidth is related to the intensity through the lineshape function because the computation of the linewidth depends on the lineshape function.

Individual ESR signals usually have two familiar shapes (Poole 1983) - the Lorentzian and Gaussian shapes the former is due to a homogeneous broadening mechanism in which all dipoles experience the same static plus time average magnetic fields but different instantaneous magnetic fields. The latter corresponds to the case of inhomogeneous broadening in which the dipole is subjected to slightly different effective magnetic fields so that at a given time only a small fraction of the spins is in resonance as the external magnetic field is swept through the line. Thus the observed signal is a superposition of a large number of slightly staggered individual 'spin packets' or an envelope. An inhomogeneous magnetic field or anisotropic interactions ( $g$ and hyperfine structure) in materials like glasses and ceramics or unresolved hyperfine structure results in Gaussian shapes. Metal clusters and conduction electrons in metals give Lorentzian and the Dysonian ESR lines respectively, while defects and free radicals in insulators give Gaussian lines. Normalized plots of Lorentzian and Gaussian lineshapes are shown in figure 13 (Sunandana 1975), while the mathematical expressions for possible lineshapes are given in table 5. Lorentzian lines are broader than those broadened by $T_{1}$. A relaxation time $T_{2}$ could be related to the width of the normalized line, in the absence of saturation, as

$$
\frac{1}{T_{2}}=\left\{\begin{array}{l}
\text { shape } \\
\text { constant }
\end{array}\right\}\left\{\begin{array}{l}
\text { gyromagnetic } \\
\text { ratio }
\end{array}\right\}\left\{\begin{array}{l}
\text { linewidth at } \\
\text { half height }
\end{array}\right\}
$$

where the shape constant is unity for Lorentzian and $\sqrt{\pi e \ln 2}$ for Gaussian lines. $1 / T_{2}$ being a linear function of linewidth, includes 'lifetime' or $T_{1}$ broadening and other homogeneous mechanisms characterized by $T_{2}^{\prime}$ so that,

$$
\frac{1}{T_{2}}=\frac{1}{T_{2}^{\prime}}+\frac{1}{2 T_{1}}
$$

$T_{2}^{\prime}$ is the spin-spin relaxation time, usually $\ll T_{1}$ for free radicals. Lines are also broadened/narrowed by exchange interactions between paramagnetic centres in concentrated spin systems. The relaxation times $T_{1}$ and $T_{2}^{\prime}$ often decide the temperatures at which ESR would become observable.

6.1d The intensity of ESR spectrum: The integrated intensity of the ESR spectrum recorded in the absorption mode or the double integrated intensity of the ESR spectrum recorded in the first-derivative mode is proportional to the density of paramagnetic centres in the sample and to the net paramagnetic susceptibility of the sample.

Assuming that (a) ESR lineshape is Lorentzian, (b) the Curie law (i.e. $\chi \propto 1 / T$ ) is applicable, (c) the microwave power applied to the sample does not saturate the resonance, and (d) the signal-to-noise ratio of the ESR cavity is unity, the ESR detection sensitivity or the minimum number of paramagnetic centres detectable in ESR $\left(N_{\text {min }}\right)$ Feher (1959) is given by

$$
N_{\min }=\frac{3 V_{c} k T_{s} \Gamma}{2 \pi g^{2} \mu_{\mathrm{B}}^{2} S(S+1) H_{\mathrm{L}} Q_{\mathrm{u}}^{\prime}}\left(\frac{F k t_{\mathrm{u}} b}{P_{0}}\right)^{\mathrm{i} / 2} \text {, }
$$

where $V_{\mathrm{c}}$ is the volume of the rectangular cavity operated in the $\mathrm{TE}_{102}$ mode; $k$ the Boltzmann constant; $T_{s}$ the sample temperature; $\Gamma$ the half width at half maximum (Gauss) of the absorption line; $H_{\mathrm{r}}$ the magnetic field at the centre of the absorption line; $Q_{u}^{\prime}$ the effective unloaded $Q$ factor of the cavity $=Q_{\varepsilon} Q_{\mathrm{v}} /\left(Q_{\varepsilon}+Q_{\mathrm{u}}\right)\left(Q_{\varepsilon}\right.$ the dielectric $Q, Q_{\mathrm{u}}=$ unloaded $\left.Q\right) ; T_{\mathrm{d}}$ the detector temperature; $b$ the bandwidth $(\mathrm{Hz})$ of the entire detecting and amplifying system; $P_{0}$ the microwave power $(\mathrm{erg} / \mathrm{s})$ incident on the cavity; $F$ the noise figure (due to sources other than thermal detector) $=1$ for an ideal spectrometer.

Typically, $\quad Q_{\mathrm{u}}^{\prime}=5000, T_{\mathrm{s}}=T_{\mathrm{d}}=300 \mathrm{~K}, \quad H_{\mathrm{r}}=3400$, $\Gamma=0.1 \mathrm{mT}, g=2.00, S=1 / 2, V_{\mathrm{c}}=11 \mathrm{cc}$ (X-band, $\mathrm{TE}_{102}$ cavity); $F=100 ; b=1 \mathrm{~Hz} ; P_{0}=10^{6} \mathrm{erg} / \mathrm{s}=100 \mathrm{~mW}$; so that $N_{\min } \approx 10^{11}$ ('Worst case sensitivity') (Wertz and Bolton 1972).

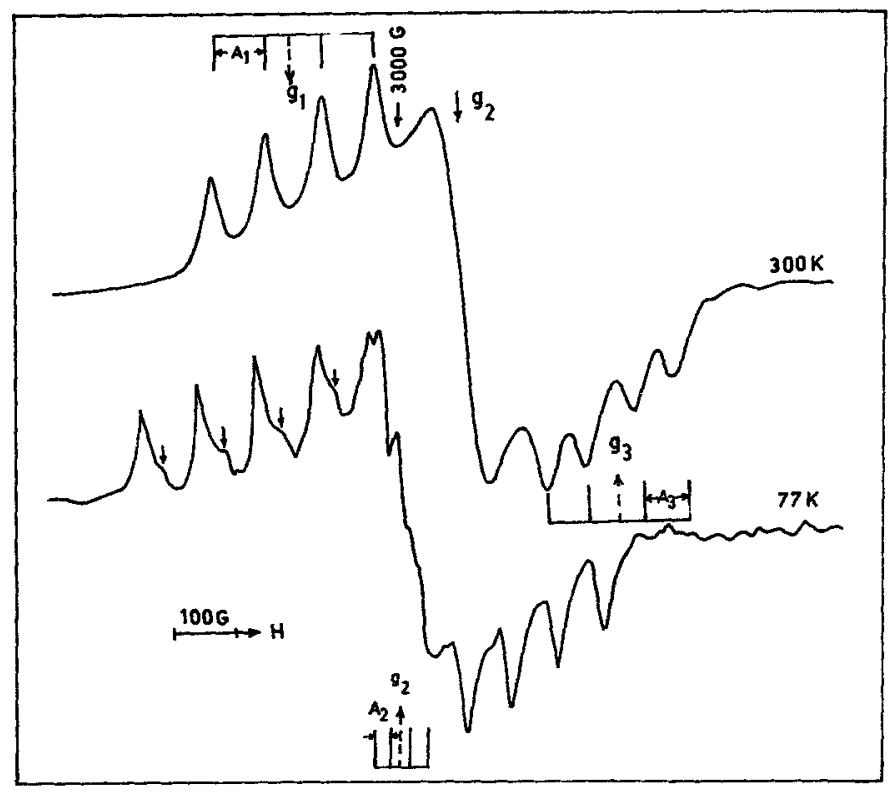

Figure 12. EPR spectrum of a typical paramagnetic centre $\mathrm{Cu}^{++}$in $\mathrm{KHSO}_{4}$ powder - located at a site of rhombic symmetry. The principal $g\left(g_{1}, g_{2}, g_{3}\right)$ and $A\left(A_{1}, A_{2}, A_{3}\right)$ values are indicated. Note that the components of $A_{2}$ are resolved at $77 \mathrm{~K}$. The spectrum arises from $\mathrm{Cu}^{++}\left(3 \mathrm{~d}^{9}, s=1 / 2\right)$ interacting with $\mathrm{Cu}$ nucleus $(I=3 / 2)$ (Bruker Almanac 1993). 
The absolute intensity of the observed ESR signal is determined by: (i) the area under the absorption curve or the double integrated first derivative curve $(A)$, (ii) modulation amplitude $(M)$, (iii) amplitude of the magnetic component of microwave field $\left(H_{1}\right)$ at the sample. $H_{1}$ is a function of $P_{0}, Q_{u}^{\prime}$ and its own distribution within the cavity, (iv) the receiver gain of the spectrometer $(G)$, (v) sample temperature, (vi) $g$-factor, (vii) microwave frequency, and (viii) filling factor $(\eta) . \eta \sim 2 V_{s} / V_{c}$, where $V_{s}$ is the sample volume and $V_{c}$ the cavity volume.
Assuming that the geometry of the sample and standard are the same, so that $H_{1}$ is the same for both, and that neither the standard nor sample signal saturates, the concentration $[x]$ of the paramagnetic species in the test sample is given, relative to that of the standard [std], by

$$
[x]=\frac{[\operatorname{std}] A_{\mathrm{x}} G_{\mathrm{s}} M_{\mathrm{s}} g_{\mathrm{s}}^{2}[S(S+1)]_{\mathrm{std}}}{A_{\mathrm{s}} G_{\mathrm{x}} M_{\mathrm{x}}\left(g_{\mathrm{x}}\right)^{2}[S(S+1)]_{\mathrm{x}}},
$$

provided the scanning of the magnetic field is done in

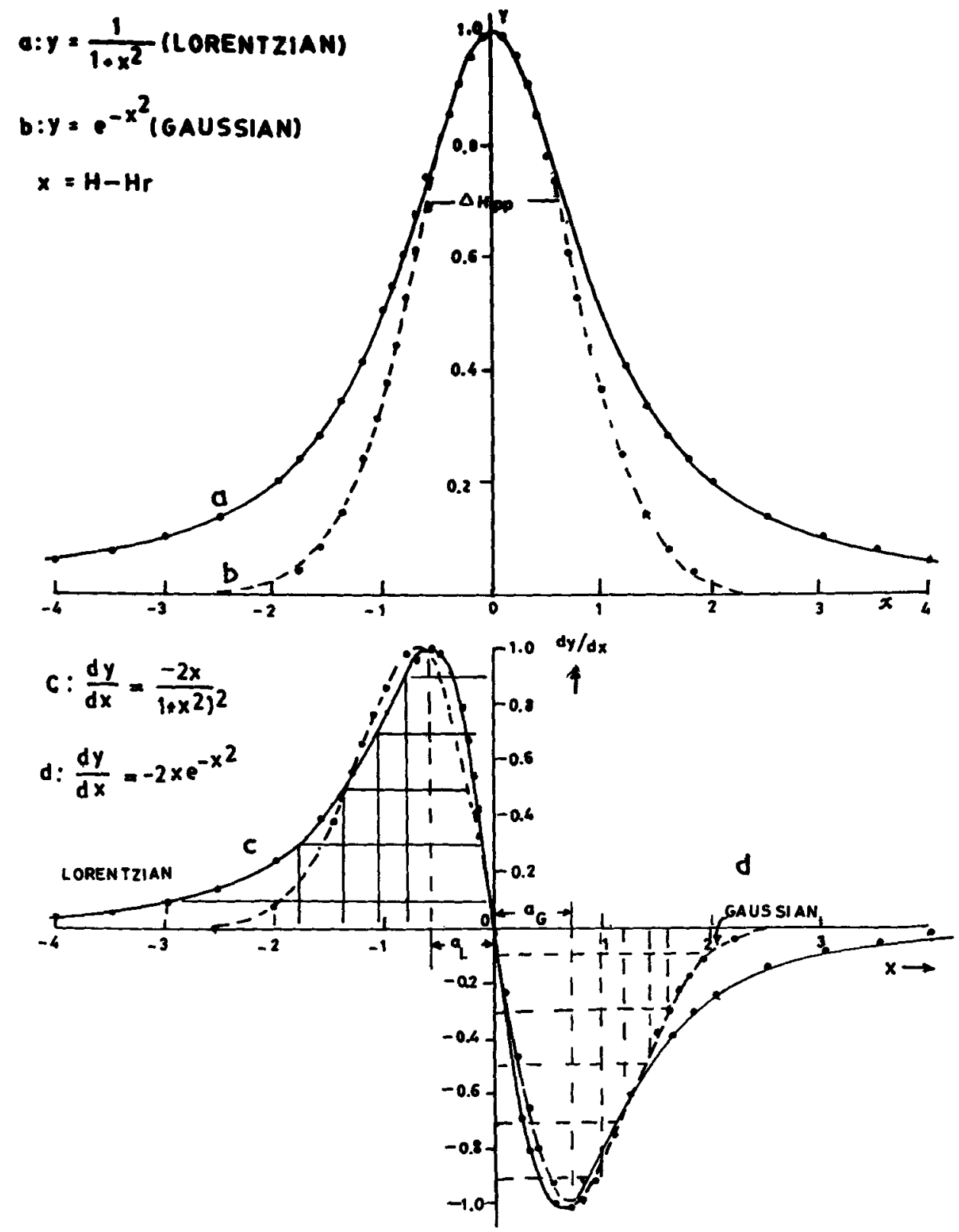

Figure 13. Normalized ESR lineshapes. (a) Lorentzian absorption, (b) Gaussian absorption, (c) Lorentzian first-derivative, (d) Gaussian first-derivative $a_{L}$ and $a_{0}$ are the basic peakto-centre linewidths for Lorentzian and Gaussian profiles in terms of which the 'widths' corresponding to $10,30,50,70$ and $90 \%$ of maximum are measured, for determination of shape of a test ESR spectrum (McMillan and Halpern 1971). 
Table 5. ESR lineshapes.

Absorption lineshape (and linewidth)
First derivative absorption

Linewidth

(fullwidth at half

maximum) $\Delta H_{1 / 2}$

Area

$$
\frac{\mathrm{d} Y^{\mathrm{L}}}{\mathrm{d} H}=\frac{16 y_{\mathrm{m}^{\prime}} \frac{\left(H-H_{\mathrm{p}}\right)}{\frac{1}{2} \Delta H_{\mathrm{pp}}}}{3+\left[\frac{\left(H-H_{\mathrm{p}}\right)}{\frac{1}{2} \Delta H_{\mathrm{pp}}}\right]^{2}}
$$

$y_{\mathrm{m}^{\prime}}=$ total vertical height

$H_{\mathrm{r}}=$ resonance field

$\Delta H_{\text {pp }}=$ peak-to-peak width

$$
\begin{aligned}
& \frac{\mathrm{d} Y^{\mathrm{G}}}{\mathrm{d} H}=y_{\mathrm{m}^{\prime}}\left[\frac{H-H_{\mathrm{r}}}{\frac{1}{2} \Delta H_{\mathrm{pp}}}\right] \\
& \exp \left[-\frac{1}{2}\left\{\left(\frac{H-H_{\mathrm{r}}}{\frac{1}{2} \Delta H_{\mathrm{pp}}}\right)^{2}-1\right\}\right]
\end{aligned}
$$

$$
\sqrt{3} \Delta H_{\mathrm{pp}} \quad \frac{2 \pi}{\sqrt{3}} Y_{\mathrm{m}^{\prime}}\left(\Delta H_{\mathrm{pp}}\right)^{2}
$$

$y_{\mathrm{m}}=$ maximum amplitude

$\Delta H_{1 / 2}=$ half-amplitude linewidth

$Y_{\mathrm{c}}^{\mathrm{e}}=Y_{\mathrm{m}} \exp \left[-0.693\left[\frac{H-H_{\mathrm{f}}}{\frac{1}{2} \Delta H_{1 / 2}}\right]^{2}\right]$

Voigt

$Y^{\mathrm{V}}=\frac{\sqrt{\ln 2}}{\pi}\left(\frac{\Delta H_{1 / 2}^{\mathrm{L}}}{\Delta H_{1 / 2}^{\mathrm{G}}}\right) x$

$\int_{-\alpha}^{a} \frac{e^{-x^{2}} \mathrm{~d} x}{\left(\frac{\Delta H_{1 / 2}^{\mathrm{L}}}{\Delta H_{1 / 2}^{\mathrm{G}}}\right)^{2} \ln 2+\left(2 \sqrt{\ln 2}\left[\frac{H-H_{\mathrm{r}}}{\Delta H_{1 / 2}^{\mathrm{C}}}-x\right]^{2}\right)}$

$\Delta H_{p p}^{\mathrm{v}}$ (observed) $=\frac{\Delta_{\mathrm{G}}^{2}+0.9085 \Delta_{\mathrm{G}} \Delta_{\mathrm{L}}+0.4621 \Delta_{\mathrm{L}}^{2 s}}{\Delta_{\mathrm{G}}+0.4621 \Delta_{\mathrm{L}}}$

$\Delta_{L}, \Delta_{G}=$ Assumed Lorentzian and Gaussian half-widths

Dysonian

$Y^{\mathrm{D}}=-\left[\frac{\omega H_{1}^{2} R^{2} \omega_{0} x_{0} T_{\mathrm{r}} A \delta}{8}\right] F_{\mathrm{D}}(R, x, \chi, \eta)^{*}$ $\sqrt{2 \ln 2} \cdot \Delta H_{\mathrm{pp}} \quad(2 \pi e) Y_{\mathrm{m}^{\prime}}\left(\frac{1}{2} \Delta H_{\mathrm{pp}}\right)^{2}$

Poole 1983

s'Stoneham A M 1972 J. Phys. D5 670

${ }^{*} F_{\mathrm{D}}(R, x, \chi, \eta)=\left\{\frac{R^{4}\left(x^{2}-1\right)+1-2 R^{2}}{\left[\left(R^{2} x-1\right)^{2}+R^{4}\right]^{2}}\left[\frac{2 \chi}{R\left(x^{2}+1\right)^{1 / 2}}+R^{2}(x+1)-3\right]+\frac{2 R^{2}\left(1-R^{2} x\right)}{\left[\left(R^{2} x-1\right)^{2}+R^{4}\right]^{2}}\left[\frac{2 \eta}{R\left(x^{2}+1\right)^{1 / 2}}+R^{2}(x-1)-3\right]\right\}$

where $H_{1}=$ microwave magnetic field amplitude,

$R=\sqrt{\frac{\text { spin diffusion time }\left(T_{\mathrm{D}}\right)}{\text { spin lattice relaxation time }\left(T_{\mathrm{P}}\right)}}$,

$\omega_{0}=$ Resonance frequency,

$H_{0}=$ Resonance magnetic field,

$x_{0}=$ Static paramagnetic susceptibility,

$A=$ Surface area of the (metallic) sample,

$x=\gamma T_{1}\left(H_{0}-H\right)$,

$\chi=(\operatorname{sign}$ of $x)\left\{\left(x^{2}-1\right)^{1 / 2}-1\right]^{1 / 2}$,

$\eta=\left[\sqrt{\left(x^{2}-1\right)}+1\right]^{1 / 2}$. 
the same way for standard and sample. If the lineshape of the ESR signal is accurately known (to be Lorentzian or Gaussian) then an estimate of the number of spins per unit volume $\left(N_{\mathrm{s}}\right)$ may be made using the formula

$$
N_{\mathrm{s}}=I_{\mathrm{pp}} \Delta H_{\mathrm{pp}}^{2}
$$

where $I_{\mathrm{pp}}$ is the peak-to-peak amplitude (intensity) and $\Delta H_{\mathrm{pp}}$ the peak-to-peak width of the resonance line. Kwan and Yen (1979) have given the modified, easy to apply, but exact formula

$$
N_{\mathrm{s}}=0.285 I_{\mathrm{pp}} \Delta H_{10}^{2},
$$

where $\Delta H_{10}$ is the width at the position $1 / 10$ of the peak-to-peak height of the first derivative curve.

The high frequency magnetic susceptibility involved in ESR is $\chi^{*}=\chi^{\prime}(\omega)-i \chi^{\prime \prime}(\omega)$, where $\chi^{\prime}(\omega)$ is the real part or the dispersion component, and $\chi^{\prime \prime}(\omega)$ is the imaginary or absorption component (strictly speaking $\chi^{\prime \prime}$ is also a function of magnetic field which considerably alters the description of ESR spectra (Zhong and Pilbrow 1991)). The measurement of the usually processed ESR absorption signal, proportional to $\chi^{\prime \prime} H_{1}$ or the derivative proportional to $\mathrm{d}\left(\chi^{\prime \prime} H_{1}\right) / \mathrm{d} H_{1}$, where $H_{1}$ is the strength of the microwave magnetic field, leads to the paramagnetic susceptibility of the sample. But unlike in the conventional measurement of $\chi$ using Faraday or Guoy balance or vibrating sample magnetometer there is no need to correct for the diamagnetic contribution to $\chi$ in the ESR measurement.

The absorption susceptibility is given by

$$
\chi^{\prime \prime}=P_{0}\left(2 \omega H_{1}^{2}\right)^{-1} \text {, }
$$

where $P_{0}$ is the power absorbed by the spin system when the microwave held $H_{1}$ is perpendicular to static field $H$ and $\omega=2 \pi \nu, \nu$ being the microwave frequency. For an unpaired spin system $S=1 / 2$ with isotropic $g$

$$
\chi^{\prime \prime}=\delta N \frac{1}{4} \pi g^{2} \beta^{2} \frac{f\left(\nu-v_{0}\right)}{h}
$$

where $\delta N=N(+1 / 2)-N(-1 / 2)$, the population difference between states with $M_{\mathrm{s}}=+1 / 2$ and $M_{\mathrm{s}}=-1 / 2$ and $f\left(\nu-v_{0}\right)$ is the lineshape function Gaussian for an inhomogeneously broadened line and Lorentzian for a homogeneously broadened line.

For a large $H_{1}$, the transitions induced by $H_{1}$ compete with the spin-lattice relaxation (a mechanism by which the spin system in the excited state transfers energy to the lattice or the reservoir and returns to ground state by a process characterized by a relaxation time $T_{1}$ ) in that while the former tries to make $\delta N \rightarrow 0$, the latter tends to make $\delta N \rightarrow(\delta N)_{0}$, a thermal equilibrium value of the population difference. For an inhomogeneously broadened ESR signal i.e. one due to a spin system whose resonance fields form a Gaussian envelope as due to unresolved hyperfine interactions of the spin with nearby magnetic nuclei, the steady state value of $\delta N$ is

$$
\frac{\mathrm{d}}{\mathrm{dt}}(\delta N)=0=\frac{1}{T_{1}}\left[(\delta N)_{0}-\delta N\right]-2 \delta N W,
$$

where $W$ is probability for the $M_{\mathrm{s}}=+1 / 2 \rightarrow M_{\mathrm{s}}=-1 / 2$ transition, given by $\pi^{2}\left(g^{2} \mu_{\mathrm{B}} H_{1}\right)^{2} f(0) / h^{2}$ so that

$$
\delta N=(\delta N)_{0}\left[\frac{1+2 \pi^{2}\left(g \mu_{\mathrm{B}} H_{1}\right)^{2} T_{1} f(0)}{h^{2}}\right]^{-1},
$$

and finally

$$
\begin{aligned}
\chi^{\prime \prime}\left(\nu_{0}\right)= & (\delta N)_{0} \frac{1 / 4 \pi\left(g \mu_{\mathrm{B}}\right)^{2} f(0)}{h} \\
& \times\left[\frac{1+2 \pi^{2}\left(g \mu_{\mathrm{B}} H_{1}\right)^{2} T_{1} f(0)}{h^{2}}\right]^{-1} .
\end{aligned}
$$

Thus for small $H_{1}$, the ESR absorption signal is directly proportional to $H_{1}$ while for large $H_{1}$, it is inversely proportional to $H_{1}$ (figure 14) (Sunandana 1978) due to the saturation factor or the expression in curly brackets. For measurements of spin concentration and susceptibility it is important to avoid saturation while ENDOR measurements are made on saturated ESR signals.

\subsection{Analysis of ESR spectra}

Advanced materials may be monocrystalline, microcrystalline or glassy, and, thus, give spectra that may be rich and well-resolved or poorly resolved. Single crystal ESR spectra contain maximum information by way of well-resolved and narrow lines for the paramagnetic centre trapped at several symmetry related or chemically distinct sites in the crystal lattice, with each site often having magnetic neighbours that could interact with the unpaired electron. However, ESR spectra of poly-crystalline or glassy materials often yield broad, poorly resolved/unresolved features from which one has to extract spin Hamiltonian parameters through defect modeling and computer simulation.

6.2a Monocrystalline ESR spectra: Single crystals containing ESR active species give ESR spectra that when properly analyzed give precise information about the location of the paramagnetic centre(s), its symmetry, its neighbours and sometimes even the distances between the centre and the neighbours. This information is contained mainly in the $g$-tensor and the hyperfine coupling constant tensor, the latter arising from the main magnetic 
nucleus and sometimes from the neighbouring magnetic nuclei, as in silicon alkali halides.

The task of deducing the principal $g$ - and $A$-values of the two tensors begins with the identification of three mutually perpendicular directions in the crystals (e.g. [100], [010] and [001] axes of an orthorhombic crystal). These need not always correspond to the crystallographic axes. Then ESR spectra are recorded for rotations of the crystal mounted on a specially designed goniometer (figure 15), about each of the three orthogonal axes, say $x, y$, and $z$-axes. The rotations about a given axis, say $x$-axis, i.e. in a given plane $y z$ are affected every $5^{\circ}$ or $10^{\circ}$ depending on the magnitude of the anisotropy. From the three sets of spectra arising from rotations about three axes, 'isofrequency plots' are generated, plotting the resonance magnetic field of each observed line against the orientation $\theta$ of the crystal (in a given plane) relative to the dc magnetic field. After identifying groups of lines corresponding to either chemically distinct centres or physically distinct but chemically equivalent centres, reduced plots of $g_{\mathrm{ij}}^{2}$ or $\left(g^{4} A^{2}\right)_{\mathrm{ij}}(i j=\mathrm{xy}, \mathrm{yz}, \mathrm{zx})$ vs $\theta$ are made. Thereafter each of the observed plots are fitted, by the method of least squares to the expression

$$
g_{\mathrm{ij}}^{2}(\theta)=U+V \cos 2 \theta+W \sin 2 \theta
$$

(with similar equation for $g^{4} A^{2}$ ), where

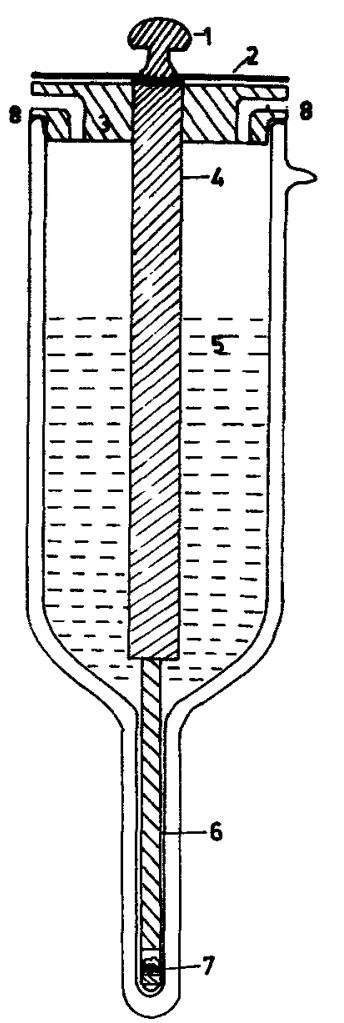

1.Rotation head (aluminium)

2. Cursor on perspex disc

3.Perspex disc graduated in degrees

4. Perspex rod

5. Liquid nitrogen

6. Tefion erystal holder

7.Crystal stuck by silicone grease

8. Escape for nitrogen vapours

Figure 14. The goniometer-in-liquid nitrogen quartz dewar for single crystal or powder ESR studies at $77 \mathrm{~K}$ (McMillan and Halpern 1971).

$$
U=\frac{g_{\mathrm{ii}}^{2}+g_{\mathrm{ij}}^{2}}{2}, V=\frac{g_{\mathrm{ii}}^{2}-g_{\mathrm{ji}}^{2}}{2} \text { and } W=g_{\mathrm{ij}}^{2}
$$

Then the elements of the $g^{2}$ or hyperfine tensor are found and the matrix formed is symmetrized, and diagonalized either directly, or using the Schonland's (1959) method. This latter method consists in accurately locating the maxima and minima, using the relation $\tan 2 \theta=W / V$ and forming the matrix elements as follows:

where

$$
\left\{g^{2}\right\}=G=\left[\begin{array}{lll}
G_{\mathrm{XX}} & G_{\mathrm{XY}} & G_{\mathrm{Xz}} \\
G_{\mathrm{YX}} & G_{\mathrm{YY}} & G_{\mathrm{YZ}} \\
G_{\mathrm{ZX}} & G_{\mathrm{ZY}} & G_{\mathrm{ZZ}}
\end{array}\right],
$$

$$
\begin{aligned}
& G_{\mathrm{XX}}=\alpha_{\mathrm{Y}}+\alpha_{\mathrm{z}}-\alpha_{\mathrm{X}}, G_{\mathrm{YY}}=\alpha_{\mathrm{z}}+\alpha_{\mathrm{X}}-\alpha_{\mathrm{Y}}, \\
& G_{\mathrm{zZ}}=\alpha_{\mathrm{X}}+\alpha_{\mathrm{Y}}-\alpha_{\mathrm{z}}, \\
& G_{\mathrm{YZ}}= \pm \sqrt{\left(\delta_{\mathrm{x}}+\alpha_{\mathrm{y}}-\alpha_{\mathrm{z}}\right)\left(\delta_{\mathrm{x}}-\alpha_{\mathrm{y}}+\alpha_{\mathrm{z}}\right)}, \\
& G_{\mathrm{ZX}}= \pm \sqrt{\left(\delta_{\mathrm{y}}+\alpha_{\mathrm{z}}-\alpha_{\mathrm{x}}\right)\left(\delta_{\mathrm{y}}-\alpha_{\mathrm{z}}+\alpha_{\mathrm{x}}\right)}, \text { and } \\
& G_{\mathrm{XY}}= \pm \sqrt{\left(\delta_{\mathrm{z}}-\alpha_{\mathrm{x}}+\alpha_{\mathrm{y}}\right)\left(\delta_{\mathrm{z}}-\alpha_{\mathrm{x}}+\alpha_{\mathrm{y}}\right)} .
\end{aligned}
$$

$\alpha$ 's and $\delta$ 's are determined by the accurately located $g_{\max }^{2}$ and $g_{\min }^{2}$ for each of the three rotations and are given by

$$
\alpha_{\mathrm{i}}=\frac{g_{\max }^{2}(\mathrm{i})+g_{\min }^{2}(\mathrm{i})}{2} \text { and } \delta_{\mathrm{i}}=\frac{g_{\max }^{2}(\mathrm{i})-g_{\min }^{2}(\mathrm{i})}{2}
$$

$\mathrm{i}=\mathrm{x}, \mathrm{y}$ or $\mathrm{z}$.

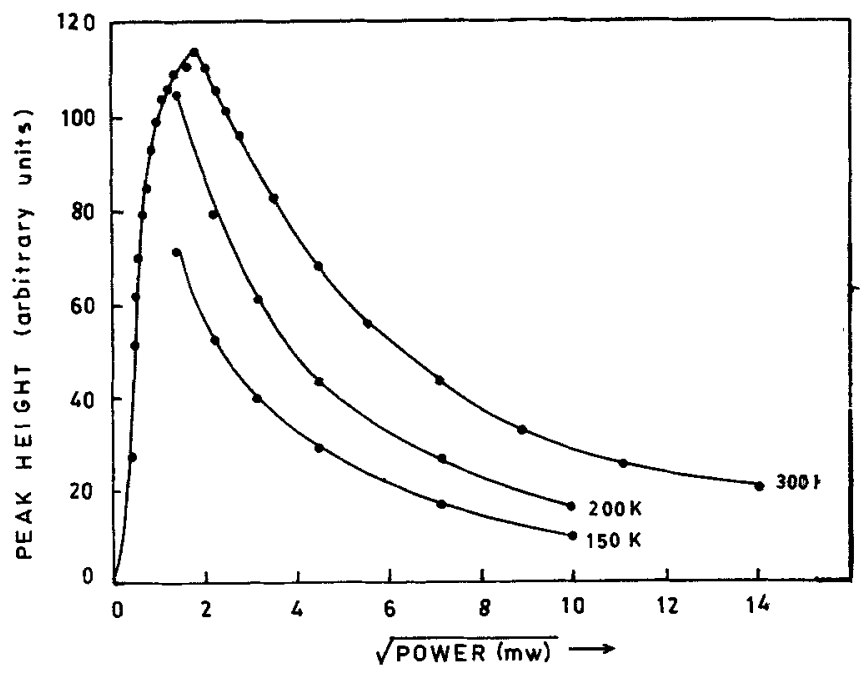

Figure 15. Microwave power saturation profile for $\mathrm{SO}_{3}^{-}$radical in $\mathrm{KHSO}_{4}$ irradiated by X-rays. Note that before saturation the peak height (intensity) varies linearly with $\sqrt{\text { power }}$ (i.e. microwave magnetic field, $H_{1}$ ), whereas after saturation, the intensity

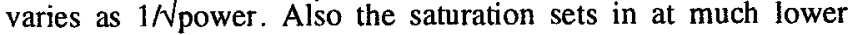
$H_{1}$ at lower temperatures (Sunandana 1978). 
If the paramagnetic centre is found at $n_{1}$ sites for rotations about $\mathrm{x}$-axis, $n_{2}$ sites for rotations about $\mathrm{y}$-axis and $n_{2}$ sites for rotations about z-axis, then one has to sort out the correct matrix elements and matrix out of a possible $n_{1}, n_{2}$ and $n_{3}$ combinations of maxima and minima. The choice can be narrowed down by seeking coincidences in the $g_{\mathrm{ij}}^{2}$ values (for example, $g_{\mathrm{xx}}^{2}$ occurs twice, once each for rotations about $y$ and z-axes) (Sunandana 1975). Finally, the diagonalization of the matrix is effected (using Jacobi method for instance) so that

$$
\begin{gathered}
{\left[\begin{array}{lll}
l_{\mathrm{xx}} & l_{\mathrm{xy}} & l_{\mathrm{xz}} \\
l_{\mathrm{xy}} & l_{\mathrm{yy}} & l_{\mathrm{yz}} \\
l_{\mathrm{xz}} & l_{\mathrm{yz}} & l_{\mathrm{zz}}
\end{array}\right]\left[\begin{array}{lll}
g_{\mathrm{xx}}^{2} & g_{\mathrm{xy}}^{2} & g_{\mathrm{xz}}^{2} \\
g_{\mathrm{xy}}^{2} & g_{\mathrm{yy}}^{2} & g_{\mathrm{yz}}^{2} \\
g_{\mathrm{xz}}^{2} & g_{\mathrm{yz}}^{2} & g_{\mathrm{zz}}^{2}
\end{array}\right]\left[\begin{array}{lll}
l_{\mathrm{xx}} & l_{\mathrm{xy}} & l_{\mathrm{xz}} \\
l_{\mathrm{xy}} & l_{\mathrm{yy}} & l_{\mathrm{yz}} \\
l_{\mathrm{xz}} & l_{\mathrm{yz}} & l_{\mathrm{zz}}
\end{array}\right]^{-1}} \\
=\left[\begin{array}{lll}
g_{\mathrm{xx}}^{2} & 0 & 0 \\
0 & g_{\mathrm{yy}}^{2} & 0 \\
0 & 0 & g_{\mathrm{zz}}^{2}
\end{array}\right] .
\end{gathered}
$$

Taking the square root of the elements of the diagonal matrix gives the principal values of $g$-tensor. The direction cosine matrix gives the directions of $g_{\mathrm{xx}}\left(l_{\mathrm{xx}}, l_{\mathrm{xy}}, l_{\mathrm{xz}}\right), g_{\mathrm{yY}}\left(l_{\mathrm{xy}}, l_{\mathrm{yy}}, l_{\mathrm{yz}}\right)$ and $g_{\mathrm{zz}}\left(l_{\mathrm{xz}}, l_{\mathrm{yz}}, l_{\mathrm{zz}}\right)$. Once the principal $g$ - and $A$-values and their direction cosines are known, the ESR centre can be located in the crystal (Atherton 1973).

Single crystal EPR spectra of transition metal compounds occasionally consist of a single structureless, perfect Lorentzian line, for certain orientation of the crystal that indicate exchange averaging of $g$ - and $A$-anisotropy and local dipolar fields. Such anomalous angular variation observed in (2,2'-bipyridine-3,3'-dicarboxylic acid) dichloro copper (II) monohydrate, in which longrange exchange interaction via lattice water was observed, has been analysed for the molecular $g$-tensor using a decoupling procedure that uses a molecular coordinate system and relates it to the crystal axes system through a coordinate transformation, in order to overcome the effect of incomplete averaging of $g$-anisotropy (Balagopala Krishna and Rajasekharan 1990).

6.2b Polycrystalline ESR spectra: Almost all varieties of advanced materials including electro-ceramics, optical materials, catalysts, polymers and glasses are composed of randomly oriented, small crystallites. The ESR spectra of such materials is the envelope of the spectra from all possible orientations of the paramagnetic centre or radical species with respect to the external magnetic field. To analyze such spectra is to extract the principal value of $\mathrm{g}$ and hyperfine structure tensors.

Consider a unit sphere (Lebedev 1963) in which the coordinates $x, y, z$ of the ESR centre is fixed while $\mathbf{H}$ is a vector whose coordinates are $\theta$ and $\phi$ (as shown in figure 16a). Every orientation of $H$ on the sphere is equally probable. The number of centres with a magnetic field orientation between $\theta$ and $\theta+\Delta \theta$ and between $\phi$ and $\phi+\Delta \phi$ are given by the solid angle

$$
\Delta \Omega=\sin \theta \Delta \theta \Delta \phi .
$$

But only a fraction of these radicals would contribute to microwave energy absorption at a magnetic field between $H$ and $H+\mathrm{d} H$, this fraction depending on the individual linewidth $\Delta H$ and the distance from resonance $H_{\mathrm{r}}-H$. A function $f\left(H_{\mathrm{r}}-H_{1} \Delta H\right)$ defines this fraction, where $H_{\mathrm{r}}$ the resonance field of the individual line is itself a function of orientation, as illustrated in figure 16b. The aim is to determine the total energy absorption at the magnetic field $H$. The intensity of this absorption at a given magnetic field is the product of the fraction defined above and the element of the solid angle, summed over all solid angles, because all orientations contribute to the absorption, in principle:

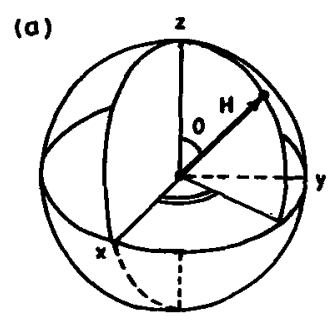

(b)

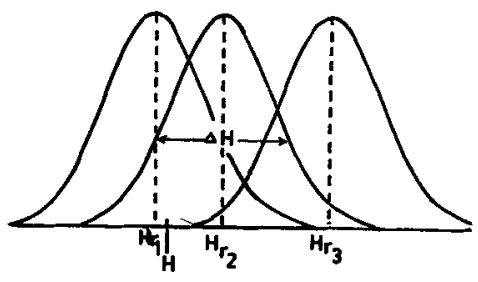

(c)

(d)
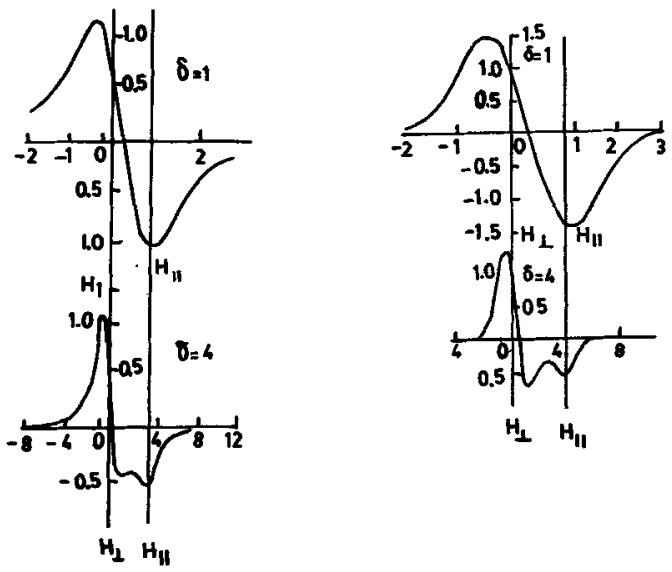

Figure 16. Analysis of powder EPR spectral shapes. a. Coordinate system showing $\mathbf{H}$ vector in a unit sphere, b. contribution of three different crystallites to the absorption at the magnetic field $H$. Note the magnitudes $H_{\mathrm{r} 2}-H_{\mathrm{r} 1}$ and $H_{\mathrm{r} 3}-H_{\mathrm{r} 2}$ relative to $\Delta H$ to $H_{\mathrm{r} 2}$, the resonance field of the second crystallite, c. theoretical shapes of asymmetric ESR lines based on Lorentzian form of individual lines and $\mathbf{d}$. theoretical shapes of asymmetric ESR lines based on Gaussian form of individual lines. Comparison with experimental spectra enable extraction of reasonably accurate $g$-parameters (Lebedev 1963). 


$$
\begin{aligned}
I & =1 / 4 \pi \sum f\left(H_{\mathrm{r}}-H, \Delta H\right) \Delta \Omega_{\mathrm{i}} \\
& =1 / 4 \pi \sum_{j=0}^{2 \pi} \sum_{i=0}^{\pi} f\left(H_{\mathrm{r}}-H, \Delta H\right) \sin \theta_{\mathrm{i}} \Delta \theta_{\mathrm{i}} \Delta \theta_{\mathrm{j}} \\
& =1 / 4 \pi \int_{0}^{2 \pi} \int_{0}^{\pi} f\left(H_{\mathrm{r}}-H, \Delta H\right) \sin \theta \mathrm{d} \theta \mathrm{d} \phi,
\end{aligned}
$$

where integral sign has replaced summation and $1 / 4 \pi$ is the normalized factor.

As the derivative absorption is usually measured

$$
\frac{\mathrm{d} I}{\mathrm{~d} H}=1 / 4 \pi \int_{0}^{2 \pi} \int_{0}^{\pi} f^{\prime}\left(H_{\mathrm{r}}-H, \Delta H\right) \sin \theta \mathrm{d} \theta \mathrm{d} \phi .
$$

The specific form of the function $f^{\prime}\left(H_{\mathrm{r}}-H, \Delta H\right)$ depends on the shape of the individual derivative curves. For Lorentzian absorption curve,

$$
\begin{aligned}
f^{\prime}\left(H_{\mathrm{r}}-H, \Delta H\right)= & \frac{16}{\pi(\sqrt{3} \Delta H)^{3}}\left(H_{\mathrm{r}}-H\right) \\
& \times\left[i+4 / 3\left(\frac{H_{\mathrm{r}}-H}{\Delta H}\right)^{2}\right]^{-2},
\end{aligned}
$$

where $H$ is the swept magnetic field and $\Delta H$ the magnetic field separation between the maximum and the minimum in the derivative curve, assumed to be constant, although it may be orientation dependent.

The spin Hamiltonian for the given situation decides the form taken by $H_{\mathrm{r}}$. If the paramagnetic centre experiences only the Zeeman interaction, and has only arc symmetry axis along $\mathrm{z}$-axis, then

$$
g_{\mathrm{xx}}=g_{\mathrm{yy}}=g_{\perp} \text { and } g_{\mathrm{zz}}=g_{\| !} \text {. }
$$

Thus

$$
H_{\mathrm{r}}=\left(h \nu_{0} / \mu_{\mathrm{B}}\right)\left(g_{\| 1}^{2} \cos ^{2} \theta+g_{\perp} \sin ^{2} \theta\right)^{-1 / 2},
$$

where $v_{0}$ is the experimental microwave frequency. Trivial solutions of the above equations occur when $\theta$ is $\pi / 2$ and 0 respectively, when

$$
H_{\mathrm{r}}^{\perp}=\frac{h v_{0}}{\mu_{\mathrm{B}}} g_{\perp} \text { and } H_{\mathrm{r}}^{\#}=\frac{w_{0}}{\mu_{\mathrm{B}}} g_{\|}
$$

which could be used to define the variable parameter $\delta=\Delta H_{\text {an }} / \Delta H_{\mathrm{i}}$ with $\Delta H_{\mathrm{an}}=\left|H_{11}-H_{\perp}\right|$ and $\Delta H_{\mathrm{j}}=0.865 \Delta H$. One could then generate a series of plots (figures $16 \mathrm{c}, \mathrm{d}$ ) to be compared with the experimental spectrum.

The case of rhombic symmetry or anisotropy occurs when $g_{\mathrm{xx}} \neq g_{\mathrm{yy}} \neq g_{\mathrm{zz}}$ (e.g. $\mathrm{CO}_{2}$ on $\mathrm{MgO}$ surface; sulphur hole centre in chalcogenide glasses). The example of glasses is quite general as there is a distribution of anisotropic $g$-values due to random local structural fluctuations, which gives

$$
g^{2}=g_{x x}^{2} \sin ^{2} \theta \cos ^{2} \phi+g_{y y}^{2} \sin ^{2} \theta \sin ^{2} \phi+g_{z z}^{2} \cos ^{2} \theta,
$$

where $\theta$ and $\phi$ relates as usual the resonance field to the principal axes of the $g$-tensor. The anisotropic linewidth that occurs in the lineshape function is expressed by a similar function

$$
\begin{aligned}
\Delta H^{-2}= & \Delta H_{\mathrm{xx}}^{-2} \sin ^{2} \theta \cos ^{2} \phi+\Delta H_{\mathrm{yy}}^{-2} \sin ^{2} \theta \sin ^{2} \phi \\
& +\Delta H_{\mathrm{zz}}^{2} \cos ^{2} \theta,
\end{aligned}
$$

where $\Delta H_{1}, \Delta H_{2}$ and $\Delta H_{3}$ are the half widths along the principal directions collinear with the corresponding ones of the $g$-tensor. Thus the simulated first derivative ESR spectrum has the general form

$$
\iint f\left(\frac{H-H_{\mathrm{r}}}{\Delta H}\right) \sin \theta \mathrm{d} \theta \mathrm{d} \phi
$$

The ESR spectrum of amorphous $\mathrm{MoS}_{3}$ has been simulated to obtain reasonably accurate $g$-values for the sulphur hole centre and $\mathrm{Mo}(\mathrm{V})$ ions surrounded by sulphur and oxygen atoms (Berger and Haddad 1991). Taylor et al (1975) have given an exhaustive account of the simulation of ESR spectra of polycrystalline solids. They have treated three cases of usually encountered powder spectra: (I) the most simple case of only the electron Zeeman term i.e. the first term in the general spin Hamiltonian

$$
\mathcal{H}=\mu_{\mathrm{B}} S \cdot g \cdot H+S \cdot A \cdot I+S \cdot D \cdot S+I \cdot Q \cdot I-\mu_{\mathrm{N}} I g_{\mathrm{N}} H,
$$

being dominant while the four other terms - hyperfine, fine structure (that occurs for paramagnetic ions with $S>1 / 2$, e.g. Mn $(S=5 / 2)$ and triplet state organic molecule $(S=1)$ ) and the much smaller quadruple and nuclear Zeeman - are neglected, with two cases (i) axial symmetry - $\left(g_{\mathrm{xx}}=g_{\mathrm{yy}}=g_{\perp}, g_{\mathrm{zz}}=g_{\|}, \quad g_{\perp}>g_{\| 1}\right)$ - consisting of a shoulder and a divergence, and (b) the completely anisotropic (rhombic case) $g_{x x}>g_{y y}>g_{z z}$, (II) the dominant first term is perturbed by the three other terms, the effect on the powder spectrum is calculated, retaining the hyperfine term, and (III) the electronic Zeeman term is of the same order as the other terms, when the entire spin Hamiltonian has to be diagonalized exactly.

Table 6 gives the readily usable analytical expressions for obtaining the powder patterns. Extra singularities occur in the spectrum for completely asymmetric case (Taylor et al 1975). 
There exists an important relation between the ESR spectra of powders and those of single crystals: the turning points in the angular dependence of resonance fields of the single crystal determine the peak positions in this spectrum of the corresponding powder. Exploiting this relationship, Van Veen (1978) has shown that the derivatives of the resonant positions with respect to the spin Hamiltonian parameters, including the orientation of magnetic fields, can be used to calculate the peak position and intensities without the need to calculate the powder spectrum completely. He has presented a calculation scheme to simulate a complete powder spectra for spins upto $5 / 2\left(\mathrm{Mn}^{2+}\right)$ and applied it to the case of $\mathrm{Fe}^{2}$ (III) substituted $\mathrm{AlOOH}$ (diaspore).

\section{Advanced materials and EPR}

The EPR technique has been applied for spin identification, spin counting, spin mapping, spin motion and spin imaging of a variety of advanced materials in their solid state including metals and alloys, elemental and compound semiconductors (crystalline and amorphous), alkali and silver halides, transition metal, rare earth and actinide compounds, electroceramics, catalysts, intercalation compounds, polymers, glasses and organic charge transfer complexes, besides superionic conductors and high temperature superconductors. The technique has proved to be specific (local probe in a definite static/ dynamic environment), sensitive (ppm level concentrations sufficient) to composition, phase and texture, accurate enough to a quantitative tool and non invasive (nonperturbing or nondestructive). Table 7 gives a list of the specific classes of materials to which the technique has been applied and the information sought.

The subsequent sections of this article focus on the following classes of materials: semiconducting materials, polymer materials, glasses and ceramics, optoelectronic and superionic materials. A section on imaging and microscopy discusses the basic principles of ESR imaging and microscopy with certain examples drawn from recent work. This is followed by a brief account of three of the emerging techniques, which when fully developed could be routinely applied to selected advanced materials. 'Spin labels' and biological materials such as iron-sulphur proteins (Hagen 1992) have not been considered in this article although they are of immense interest. Indeed, 'spin label' ESR has been established as a standard physical technique (Humphries and McConnell 1982).

\section{Semiconducting materials}

\subsection{General considerations}

Characterization of point defects - both unintentional and those induced by plastic deformation, irradiation,

Table 6. Basic expressions for generating EPR absorption spectra of powdered materials ${ }^{\dagger}$.

Type of spectrum

(a) Axially symmetric $g$-tensor, $g_{1}>g_{\| 1}$

$\left(g_{1}=g_{2}=g_{1}, g_{3}=g_{11}\right)$

Features: Divergence at $H_{\perp}=h v_{0} / g_{1} \mu_{\mathrm{B}}$

shoulder at $H_{\|}=l w_{0} / g_{\|} \mu_{\mathrm{B}}$

$v_{0}=$ microwave frequency

(b) Rhombically symmetric $g$-tensor,

$g_{1}>g_{2}>g_{3}$.

Features: Divergence at $H_{2}=w_{0} / g_{2} \mu_{\mathrm{B}}$

Shoulders at $H_{1}=h_{0} / g_{1} \mu_{\mathrm{B}}$ and $H_{3}=h_{0} / g_{3} \mu_{\mathrm{B}}$
Expressions

$$
\begin{aligned}
& S(H)=\frac{\left(h v_{0}\right)^{2}}{\mu_{\mathrm{B}}^{2} g_{\perp} \sqrt{g_{1}^{2}-g_{\|}^{2}} \cdot H^{2} \sqrt{H^{2}-\left(h \nu_{0} / g_{\perp} \mu_{\mathrm{B}}\right)^{2}}} \\
& \text { for } h_{0} / g_{\perp} \mu_{\mathrm{B}} \leq H \leq h w_{0} / g_{\|} \mu_{\mathrm{B}} \\
& S(H)=0 \text { elsewhere }
\end{aligned}
$$

$$
\begin{aligned}
& S(H)=\frac{2}{\pi} \frac{H_{1} H_{2} H_{3}}{\sqrt{H^{2}-H_{1}^{2}} \cdot \sqrt{H_{3}^{2}-H_{2}^{2}} \cdot H_{2}} \cdot K(x) \\
& \left(H_{3} \geq H \geq H_{2}\right) \\
& S(H)=\frac{2}{\pi} \frac{H H_{2} H_{3}}{\sqrt{H_{2}^{2}-H_{1}^{2}} \cdot \sqrt{H_{3}^{2}-H^{2}} \cdot H_{2}} \cdot K(1 / x) \\
& \left(H_{2} \geq H \geq H_{1}\right) \\
& S(H)=0 \text { dsewhere } \\
& k^{2}=\frac{\left(H_{2}^{2}-H_{1}^{2}\right)\left(H_{3}^{2}-H^{2}\right)}{\left(H^{2}-H_{2}^{2}\right)\left(H_{3}^{2}-H_{1}^{2}\right)} \\
& k(x)=\int_{0}^{\pi / 2} \frac{\mathrm{d} x}{\sqrt{1-k^{2} \sin ^{2} x}} \\
& k(0)=\pi / 2, K(1)=\infty
\end{aligned}
$$

${ }^{\dagger}$ Taylor et al (1975). 
chemical doping and thermal treatment during various stages of synthesis - is an important first step in the evaluation of semiconducting materials for applications such as integrated circuits, infrared photodiodes and solar cells. More specifically, the characterization of electronic defect states in a given semiconductor involves three aspects: (i) the number density of various defects (e.g. donors in crystalline Si or dangling bonds in amorphous silicon), (ii) the energy position of defect levels relative to the conduction band/valence band edge, or the energy spectrum which governs the macroscopic properties of the semiconductor, and (iii) defect wave function which gives the microscopic structure of the electronic defect, and, which enables assessment of (a) cross-sections for

Table 7. Principal types of advanced materials studied by EPR.

\begin{tabular}{ll}
\hline Type of material & \multicolumn{1}{c}{ Information sought } \\
\hline $\begin{array}{l}\text { I. Metals and metallic } \\
\text { materials }\end{array}$ & \\
$\begin{array}{l}\text { A. Ferromagnetic } \\
\text { metals and alloys }\end{array}$ & $\begin{array}{l}\text { Magnetic phase identification, mag- } \\
\text { netization, damping and magneto- } \\
\text { striction parameters }\end{array}$ \\
$\begin{array}{l}\text { a) crystalline } \\
\text { b) glassy }\end{array}$ & $\begin{array}{l}\text { Metal-insulator transitions, phase } \\
\text { purity in ceramics, microwave impe- }\end{array}$ \\
& dance and penetration depth
\end{tabular}

II. Semiconductors
a) crystalline
b) amorphous

III. Insulators

A. Inorganic
a) monocrystalline
b) polycrystalline
c) glassy Microscopic structure and electronic
information

B. Organic
a) monocrystalline
b) polycrystalline
Free radical production, identi- fication and detection, kinetics, motion of spin labels

C. Polymers
a) conducting

b) nonconducting

D. Ferroelectrics, optoelectronics and superionics

E. Catalysts optical transitions, (b) electron correlation effects, and (c) metastability of defect densities and sample preparative conditions.

In the testing of semiconductors ESR and ENDOR techniques are particularly valuable as they are sensitive to the short-range order of defect states, i.e. the location of the defect in the crystal lattice/amorphous network and its neighbourhood.

Unpaired electrons in semiconductors - both elemental (Si, Ge) and compound (III-V's: InSb, GaAs and II-IV's $\mathrm{ZnS}, \mathrm{CdS}$ ) - exist as: (i) electrons localized at isolated defects (impurity atoms), (ii) electrons in a partly filled band, and (iii) electrons in broken bonds (like inorganic/organic free radicals).

Physically, semiconductors are attractive materials for ESR investigations because: (i) they are intrinsically diamagnetic so that any impurity $(\mathrm{H}$ to $\mathrm{Ag}$ across the periodic table) could be studied without any interference from host lattice; (ii) these magnetically active impurities have limited solubility and give rise to narrow resonance lines; (iii) they have highly symmetric crystal structures (cubic/hexagonal) with very few atoms per unit cell (typically 2) with tetrahedral symmetry about each lattice point so that theoretical modelling is relatively simple; (iv) they are tetrahedrally bonded (involving hybridized atomic $s$ - and $p$-orbitals) and this covalency is manifest as (a) a reduction in the hyperfine interaction with central ion, (b) an additional hyperfine structure with central ion, and (c) a reduction in orbital contribution to $g$-factor; and (v) the rather small spin orbit interaction (especially in $\mathrm{Si}$ ) and long spin-lattice relaxation time make resonance signals in doped semiconductors easily saturable and thus ideal for ENDOR studies and 'spin mapping'.

Beginning with crystalline silicon-doped with arsenic to the present day porous silicon and low-temperature GaAs, the ESR characterization continues unabated. What follows is a brief, material-by-material description. Recent experimental data is compiled in table 8 .

\subsection{Silicon}

8.2a Donors, acceptors and impurities in crystalline Si: Studies on crystalline silicon doped with shallow donor $(\mathrm{P}, \mathrm{Ag}, \mathrm{Sb}$ and $\mathrm{Bi}$ ) and shallow acceptor $(\mathrm{B}, \mathrm{Al}, \mathrm{Ga}$ and $\mathrm{In}$ ) impurities, 3d-transition elements ( $\mathrm{V}, \mathrm{Cr}, \mathrm{Mn}$, $\mathrm{Fe}$ and $\mathrm{M}$ ), and, subject to bombardment from electrons, neutrons and $\gamma$-rays have yielded a wealth of ESR and ENDOR data, which are well documented in literature (Ludwig and Woodbury 1962; Zunger 1986). P-doped $\mathrm{Si}$ is a simple model system for the study of metalinsulator/semiconductor transition. At low $\mathbf{P}$ concentration, $\left(\sim 10^{15} / \mathrm{cc}\right)$ donors are completely isolated and as the concentration is increased to $\sim 10^{19} / \mathrm{cc}$ the strong donor-donor interaction leads to the formation of an impurity band of electrons hopping from $P$ to $P$. 


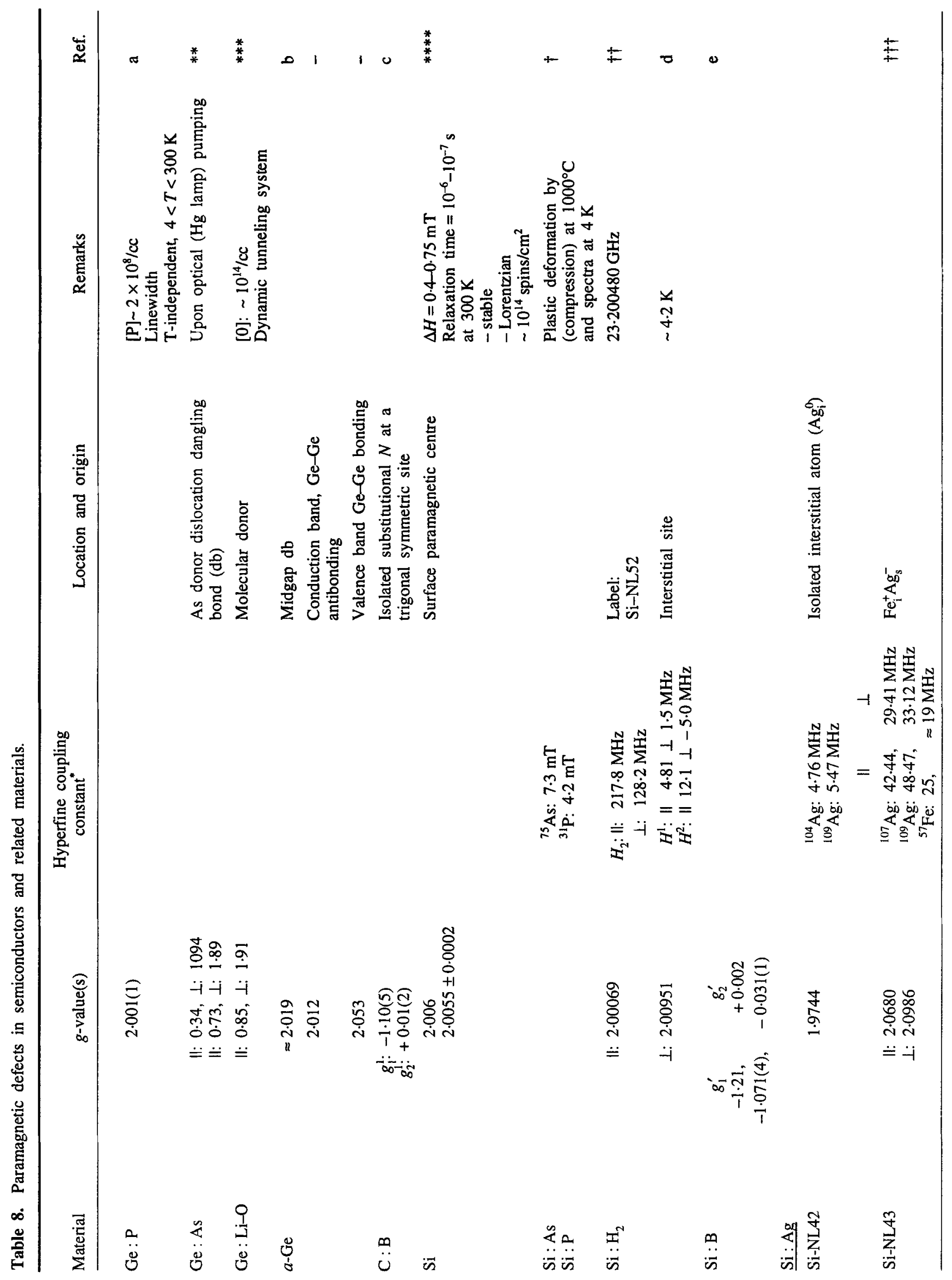


Techniques and applications of electron spin resonance

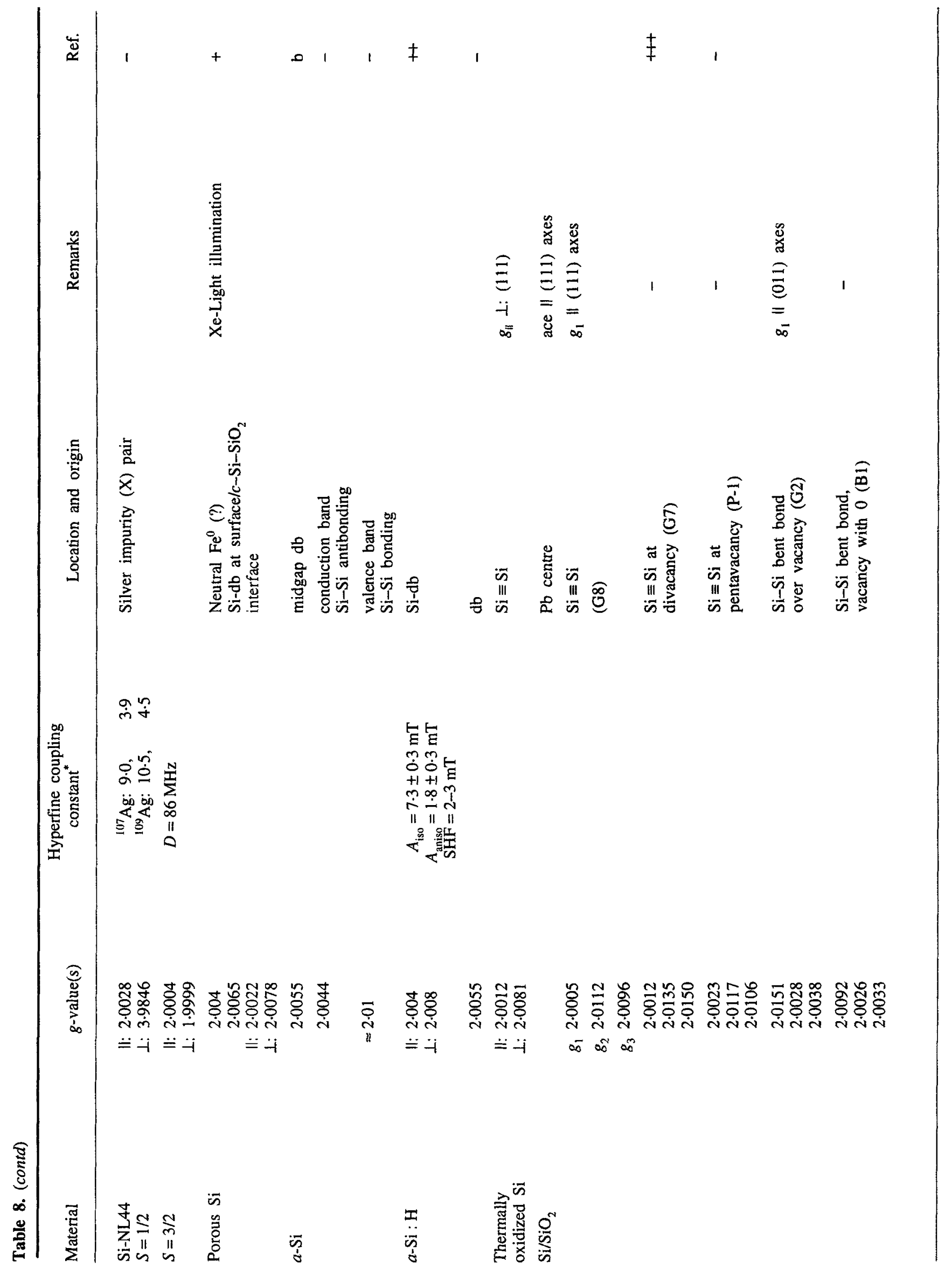




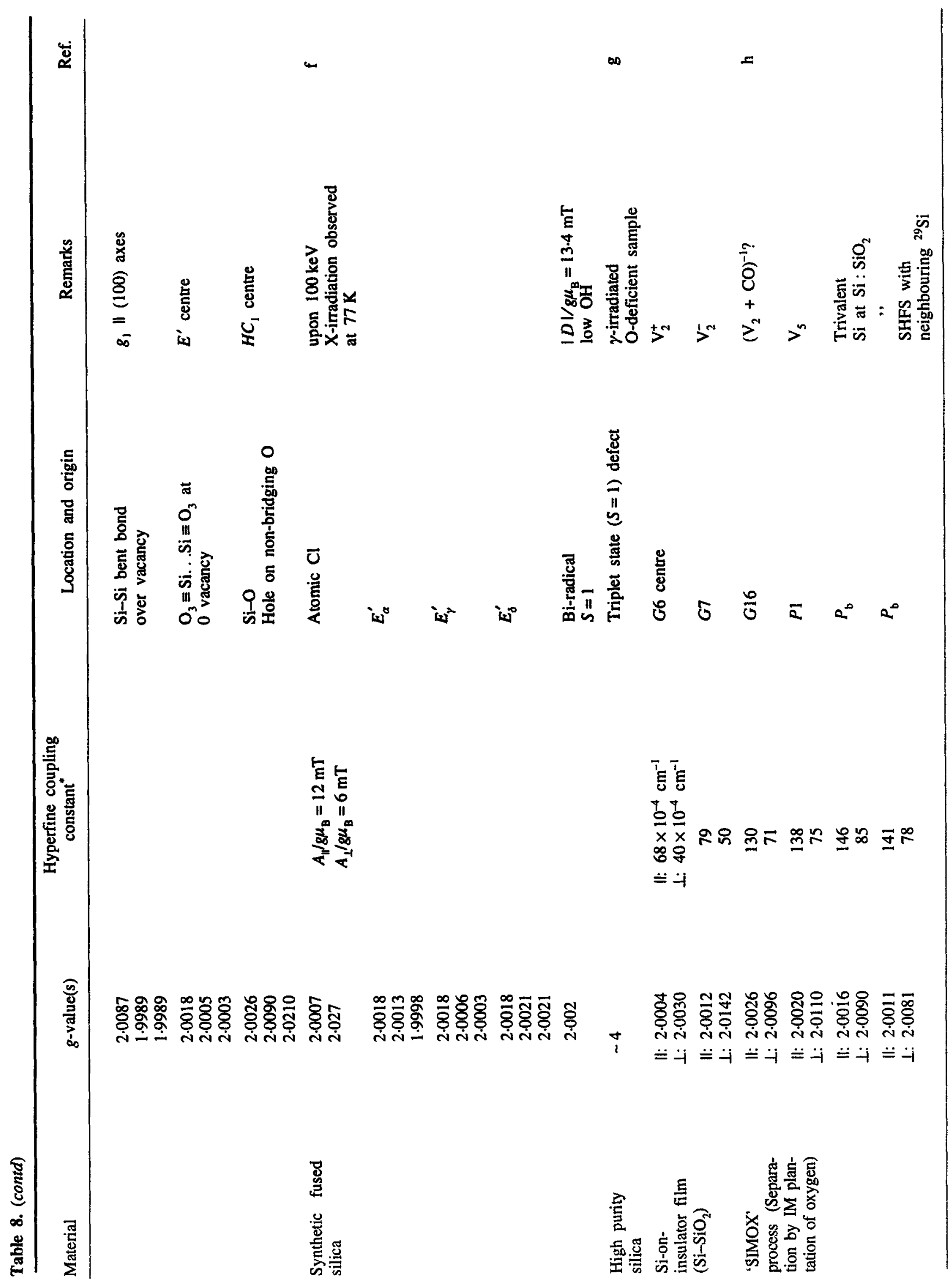




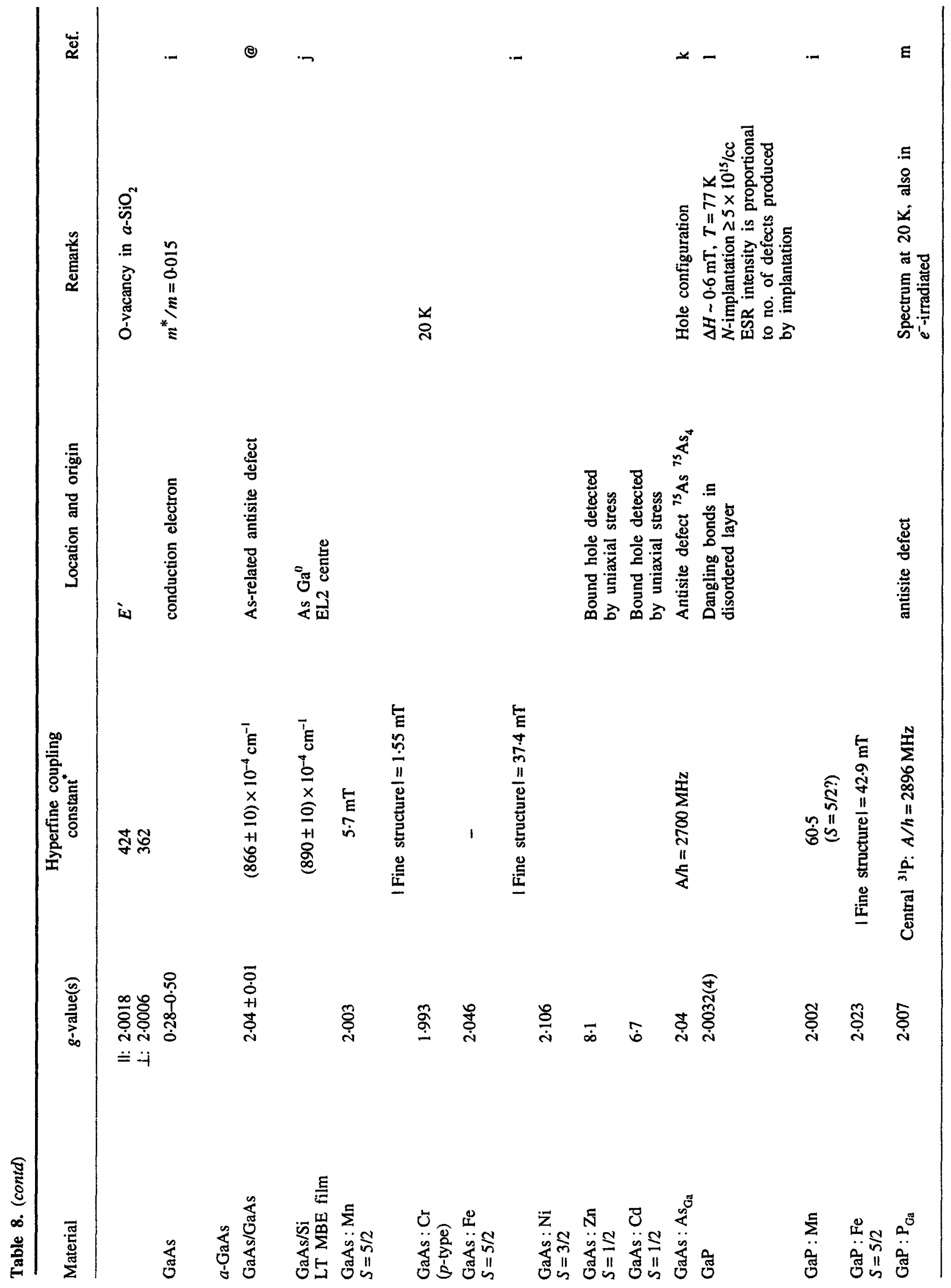




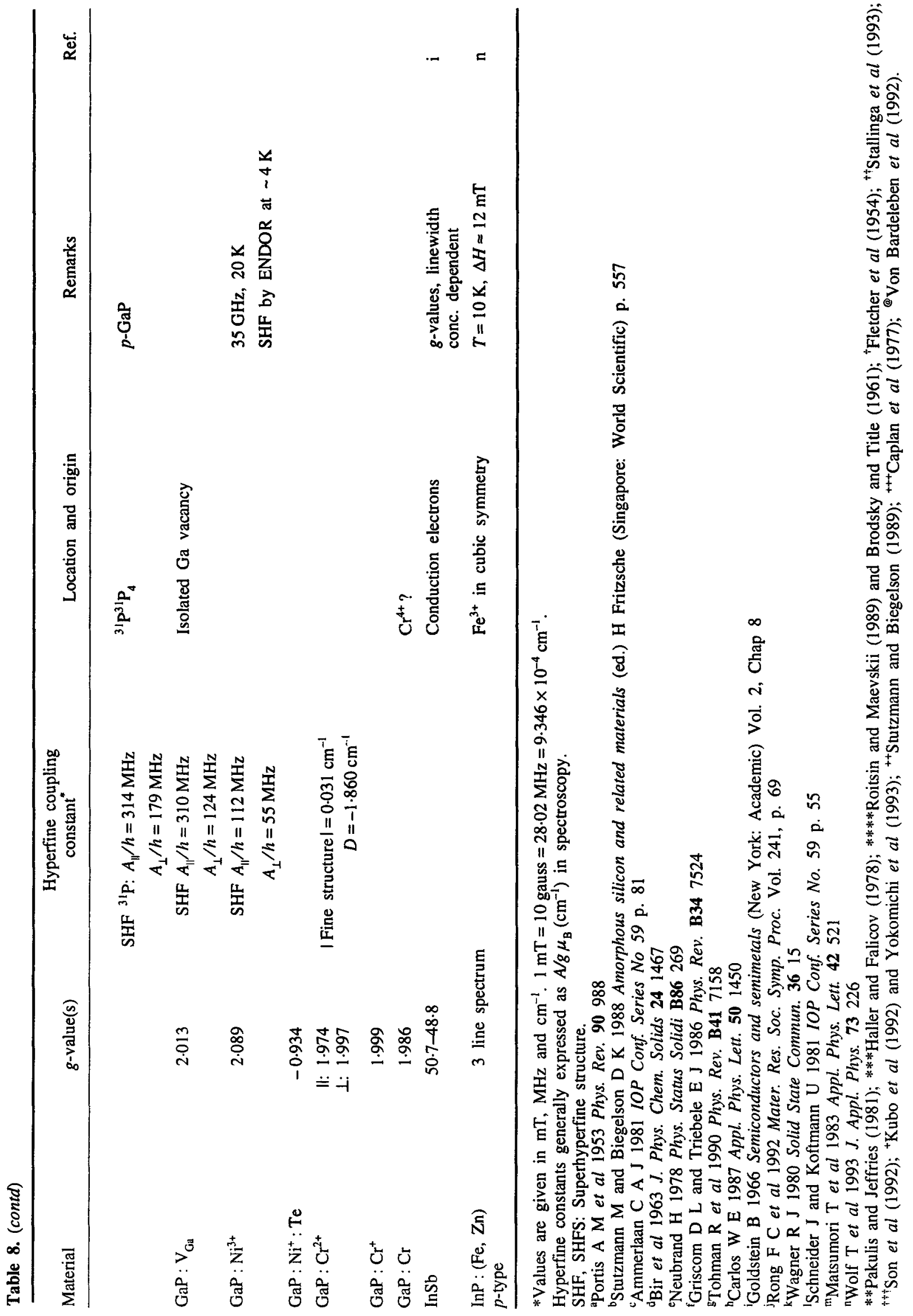


Accordingly the ESR spectrum changes from a 2-line $\mathrm{P}(I=1 / 2)$ hyperfine pattern to a $g$-shifted and broadened pattern as $P$ concentration is increased (Slichter 1971).

Three general conclusions emerge from these studies on doped $\mathrm{Si}$ :

(I) The hydrogen-like energy states available to an isolated impurity atom provide a set of localized atomic states capable of producing deep levels in the band gap of the semiconducting host.

(II) The interaction of the atomic states of an impurity at an interstitial site with the host bunches these deep levels and confines them within the gap. Thus the interstitial site 'attracts' deep levels.

(III) The substitutional site, however, 'rejects' deep levels, and is thus not favoured.

Local interatomic distances between $\mathrm{Si}$ and an $s-p$ bonded impurity can be very accurately determined using EPR hyperfine structure from magnetic ligands as has been demonstrated by Scheffler (1987) for the case of $S^{+}$in $\mathrm{Si}$. Comparing the ratio of the experimentally determined Fermi contact parameter and the dipole parameter (b) to the same ratios calculated as a function of local geometry (i.e. different S-Si bond lengths), the equilibrium geometry of the $\mathrm{Si}-\mathrm{S}$ complex is established. The $\mathrm{S}-\mathrm{Si}$ bond length thus determined is $2.345 \AA$, practically identical with the $2.35 \AA$ determined from extended X-ray absorption fine structure (EXAFS) measurements.

The role of the interstitial hydrogen impurity in semiconductors (especially $\mathrm{Si}$ ) as a passivator of deep and shallow can never be over-emphasized. It is the most fundamental and one of the most challenging issues in the materials science of silicon. Hydrogen can diffuse through Si rapidly at room temperature (unlike in metals where they can be stored). While it causes embrittlement of $\mathrm{Si}$ at low temperatures, it aids dislocation motion in $\mathrm{Si}$ at high temperatures. It can exist in $\mathrm{Si}$ as a centre with positive $\left(\mathrm{H}^{+}\right)$, neutral $\left(\mathrm{H}^{0}\right)$ and negative $\left(\mathrm{H}^{-}\right)$states (Myers et al 1992). EPR of the apparently isolated neutral hydrogen $\left(\mathrm{H}^{0}\right)$ has been observed (Gorelkinskii and Nevinnyi 1987a, b) for $\mathrm{Si}$ after proton irradiation at $80 \mathrm{~K}$. Two paramagnetic centres AA9 and AA10 - both positively charged - have been identified. AA9 shows hyperfine splitting due to $\mathrm{H}$ atoms. Both centres anneal at $180 \mathrm{~K}$.

Silver - an important $4 \mathrm{~d}$ transition metal impurity in $\mathrm{Si}$ - creates discrete levels in the forbidden gap and modifies the optical characteristics of Si. Indeed, $\mathrm{Ag}$ introduces a donor level $0.34 \mathrm{eV}$ above valence level and an acceptor level $0.54 \mathrm{eV}$ below the conduction level. An EPR study of $p$-type Si doped with Ag (Son et al 1992) has identified three paramagnetic centres: (i) an isolated interstitial silver atom in a site of trigonal symmetry in the $\mathrm{Si}$ lattice; (ii) an $\mathrm{Fe}-\mathrm{Ag}$ pair (in a sample co-doped with ${ }^{57} \mathrm{Fe}$ ) $\mathrm{Fe}_{\mathrm{i}}^{+} \mathrm{Ag}_{5}^{-}$with $\mathrm{Fe}$ in an inter- stitial position and $\mathrm{Ag}$ in a substitutional position (figure 17), and (iii) an Ag-transition metal pair.

In briefly annealed $\left(250^{\circ} \mathrm{C}, 15 \mathrm{~min}\right) \mathrm{Si}$ single crystal samples implanted with $30 \mathrm{MeV}$ hydrogen and deuterium (to $\sim 10^{17} / \mathrm{cm}^{2}$ ) an EPR spectrum attributed to a hydrogen molecule oriented along $\langle 111\rangle$ direction in the Si crystal, has been detected, in experiments performed at X- and $\mathrm{K}$-band frequencies, at liquid He temperatures (Stallinga et al 1993). The hyperfine structure of $\mathrm{H}$ is clearly reduced at $\mathrm{K}$-band as a triplet and a singlet and the $\mathrm{N}$ L52' spectrum is described by the spin Hamiltonian

$$
\mathscr{H}=\mu_{\mathrm{B}} H \cdot g \cdot S+S \cdot A_{\mathrm{H}_{2}} \cdot H_{\mathrm{H}_{2}}+S \cdot A_{\mathrm{H}^{\prime}} \cdot I_{\mathrm{H}^{\prime}}+S \cdot A_{\mathrm{H}^{2}} \cdot I_{\mathrm{H}^{2}},
$$

where $S=1 / 2, I_{\mathrm{H} 2}=1$ for the triplet spectrum and $I_{\mathrm{H} 2}=0$ for the singlet spectrum and $I_{\mathrm{H}^{\prime}}=t_{\mathrm{H}^{\circ}}$. Here $\mathrm{H}_{2}$ is the orthohydrogen molecule, while $\mathrm{H}^{1}$ and $\mathrm{H}^{2}$ are ligand hydrogens. The $g$ and $A$ parameters are included in table 8. The capture of mobile $\mathrm{H}^{-}$at the interstitially located $\mathrm{H}^{0}$ is proposed as the probable mechanism of formation of $\mathrm{H}_{2}$.

The nonobservation of $\mathrm{Au}^{0}$. in semiconducting through EPR had been as intriguing as the nonobservation of $\mathrm{Cu}^{++}$EPR in high $T_{\mathrm{c}}$ superconductors (Mehran and Anderson 1988). But from Zeeman effect studies at $1.9 \mathrm{~K}$, Watkins et al (1991) have established that both the single donor and the single acceptor levels of gold in $\mathrm{Si}$ arise from isolated substitutional neutral gold. Its ground state is paramagnetic $S=1 / 2$ and is tetragonally distorted with $g_{\|} \simeq 2.8$ and $g_{\perp} \approx 0$, unlike the isoelectronic $\mathrm{Pt}^{-}$, for which $S=1 / 2, g_{\| 1} \simeq 2 \cdot 1$ and $g_{1}=1 \cdot 4$. The absence of $\mathrm{Au}^{\circ} \mathrm{EPR}$ in $\mathrm{Si}$ is thus a direct consequence of $g_{\perp}=0$, which implies that $M_{s}$ values for the ground state quantize along the defect tetragonal axis independent of the orientation of the magnetic field. Thus there are no magnetic field dependent off-diagonal terms in the spin Hamiltonian, and no $\Delta M_{\mathrm{s}}= \pm 1$ transitions can be induced by a microwave field.

An interesting EPR and ENDOR study by Van Kemp et al (1987) of interstitial $\mathrm{Cr}^{+}$centres in $\mathrm{Si}, \mathrm{Cr}_{i}^{+}$, serves to illustrate the application of the latter, as well as to demonstrate the success of the model developed by Ludwig and Woodbury (1962) to account for the observed effective spin and $g$-values of the 3d-transition metal impurity in Si. According to their model, the introduction of a $3 \mathrm{~d}$ metal on an interstitial site with tetrahedral symmetry (figure 18) causes a splitting of the 5-fold orbitally degenerate atomic d-levels into a 3 -fold degenerate $\left(t_{2}\right)$ and a 2 -fold degenerate $(e)$ states. These levels are then filled by electrons according to Hund's rule. Significantly, the $4 \mathrm{~s}$ electrons are not used for bonding to the Si nearest neighbours and are transferred to the $3 \mathrm{~d}$ shell, leading to 'core polarization'. $\mathrm{Cr}_{\mathrm{i}}$ has $3 \mathrm{~d}^{5}$ configuration and a $t_{2}^{3} e^{2}\left({ }^{6} A_{1}\right)$ ground state. The 
existence of a donor level in $\mathrm{Cr}_{i}^{\mathrm{n} /+}$ was established, from EPR to be $0.22 \mathrm{eV}$ below the conduction level. Intriguingly, the observed hyperfine interaction between $3 \mathrm{~d}$ electrons and the impurity nucleus is smaller than that
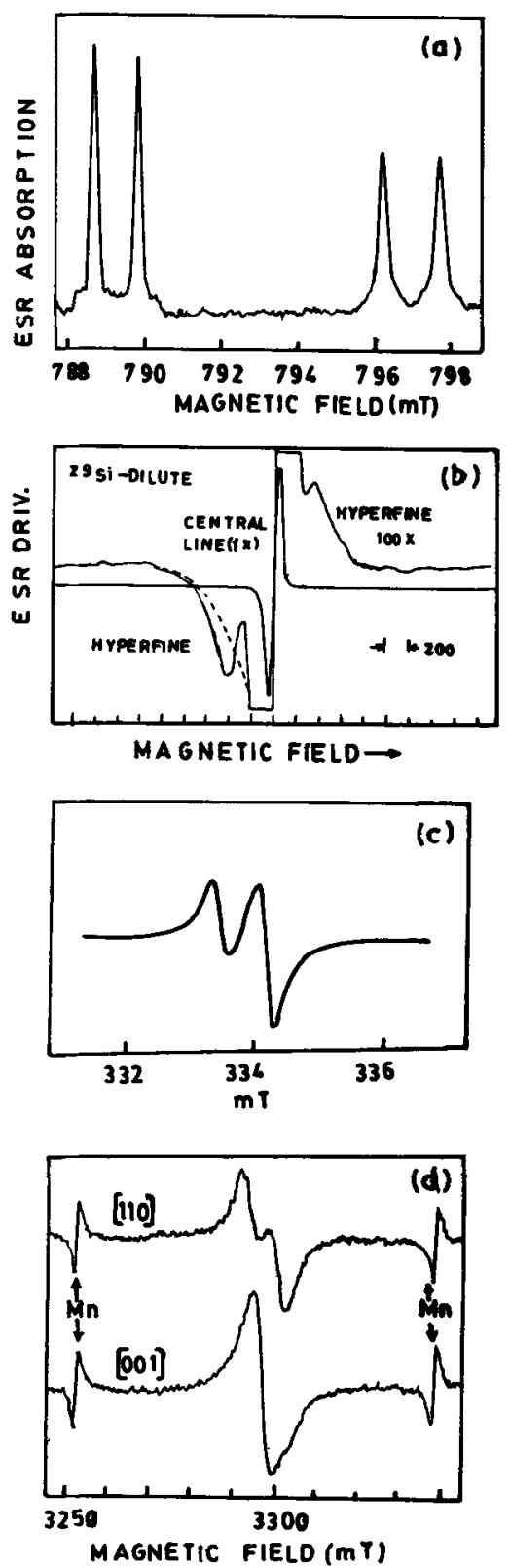

Figure 17. Typical ESR spectra of transition metal impurities and structural defects in different types of silicon. a. Spectrum of the 'Si-NL 43' centre in Ag-doped silicon showing hyperfine structure due to ${ }^{109} \mathrm{Ag}$ nucleus $(I=1 / 2)$ measured at $23 \cdot 182$ $\mathrm{GHz}$, with magnetic field along [011] axis, b. spectrum of $a$-Si : $\mathrm{H}$ containing naturally abundant ${ }^{29} \mathrm{Si}$ showing hyperfine structure of the $g=2.0055$ resonance and the central line is obtained at low gain, c. spectrum of porous silicon annealed for $30 \mathrm{~min}$ in nitrogen atmosphere at $350^{\circ} \mathrm{C}$ and d. angular dependence of ESR spectra of porous silicon crystal when the magnetic field is along [110] axis and normal to the axis i.e. [001] when the crystal is rotated in the [110] plane (Son et al 1992; Stutzmann and Biegélson 1989; Yokomichi et al 1993). calculated for the free $\mathrm{Cr}_{\mathrm{i}}^{+}$ion which would imply a rather large delocalization of the $3 d$ wave functions, leading to a large hyperfine interaction with the $\mathrm{Si}$ ligands. But these interactions were not observed in EPR (figure 19a). ENDOR experiments have revealed (figure 19b), that in $\mathrm{Si}: \mathrm{Cr}_{\mathrm{i}}^{+}$, the impurity electrons interact with one hundred and two (102) $\mathrm{Si}$ atoms distributed in 9 shells surrounding the $\mathrm{Cr}$ impurity. An important result that emerges from this study is that at least $22 \%$ spin density is transferred from the impurity to the host crystal. This has resolved the contradiction that despite $52 \%$ delocalization of the impurity wave function worked out on the basis of EPR data, proportionately large hyperfine interaction due to $\mathrm{Si}$ ligands is not observed.

8.2b Deformed and implanted Si: Plastically deformed Si gives rich EPR spectra due to defect clusters of the vacancy type, produced during deformation, similar to those induced by radiation damage. Their $g$-tensors of the dangling bond type - almost axially symmetric with $g_{\perp}-g_{\mathrm{e}}>0$ and $g_{\|} \approx g_{\mathrm{e}}$. These centres occur in all crystallographically equivalent orientations with comparable intensity (Webb and Alexander 1997).

Ion implantation, an important technique of device fabrication, is a process in which foreign atoms (say ${ }^{75} \mathrm{As}$ or ${ }^{76} \mathrm{As}$ ) are introduced in, say $\mathrm{Si}$ wafer or $\mathrm{Si}$ substrate, and, occur at relatively low temperatures such as room temperature substrate. Heavy-dose $\left(\sim 10^{15} / \mathrm{cm}^{2}\right)$ ion implantation leads to the formation of amorphous layers in semiconductors (Masuda 1977) which can be annealed at low temperatures. It is characterized by an

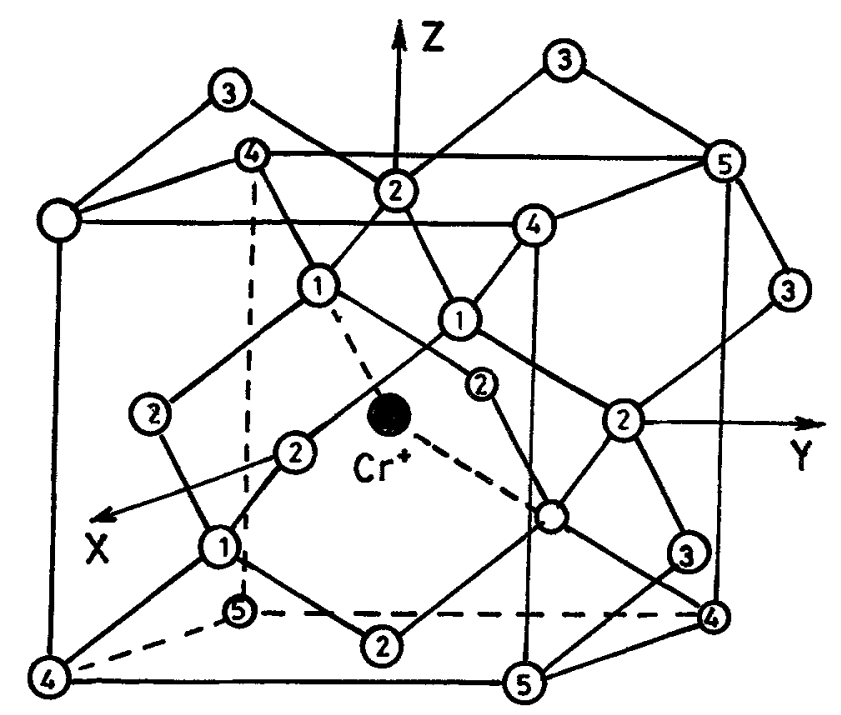

Figure 18. Silicon crystal lattice showing $\mathrm{Cr}^{+}$in an interstitial position formed by four Si-tetrahedra. The numbers $1,2,3,4$ and 5 denote the closest, next nearest ... shells relative to the impurity. The $x-y-z$ coordinates provide a reference for hyperfine interaction tensors to be derived from ENDOR spectra (Webb and Alexander 1977). 
isotropic Lorentzian-shaped ESR signal with $g=2.006$ and $\Delta H_{\text {max slope }}=0.48-0.6 \mathrm{mT}$ which when annealed at $\sim 300^{\circ} \mathrm{C}$ yields the ideal amorphous state. Annealing around $500-550^{\circ} \mathrm{C}$ produces ESR due to conduction electrons with $g=1.998-1.999$ and complete recovery of the charge carriers. The important result is that the structure of voids in the amorphous layer produced by heavy-ion implantation has inhomogeneous characteristics within the void, in contrast to that in clean surfaces cleaved in ultra-high vacuum.

In heavily P-doped $\left(7 \times 10^{17}\right.$ atoms $\left./ \mathrm{cm}^{3}\right) \mathrm{Si}$, upon $1.5 \mathrm{meV}$ electron irradiation at room temperature, twophosphorus defect complexes are detected in EPR (Sieverts and Ammerlaan 1977) whose formation is explained by a slow diffusion of the phosphorus-vacancy pairs or $\mathrm{E}$ centres during irradiation at $60^{\circ} \mathrm{C}$ and subsequent trapping by other phosphorus atoms. An ionization-induced diffusion mechanism is found to be essential for this process.

8.2c Amorphous silicon (a-Si) and hydrogenated amorphous silicon $(a-S i: H)$ : The main difference between crystalline and amorphous silicon is that in the latter the edges of the valence and conduction bands are somewhat ill defined, so that the 'tails' of these two bands consist of states that exhibit a certain degree of localization. Thus these 'localized states' occur in the band gap and give rise to characteristic EPR signals, attributed to point defects.

The most widely investigated centre in undoped $a-\mathrm{Si}$ (and $a-\mathrm{Si}: \mathrm{H}$ ) is the so-called $\mathrm{D}$ centre due to Si dangling bonds, which are actually unbonded orbitals on a 3coordinated (instead of the usual 4-coordinated) neutral silicon atom $\left(\mathrm{Si}_{3}\right)$ in the disordered network. Due to lack of bonding partners, the fourth atomic $s p^{3}$ hybrid is unbonded and thus the electronic defect is named 'dangling band', analogous to similar defect states at the surfaces/grain boundaries of crystalline silicon. The fingerprint of this electronic defect, which, in undoped $a-\mathrm{Si}: \mathrm{H}$ essentially determines the lifetime of excess charge carriers and thus limits the performance of $a-\mathrm{Si}: \mathrm{H}$ thin films for many applications, is a characteristic ESR signal with $g=2.0055$ whose intensity is a convenient measure of the quality of a given $a-S i$ specimen.

The attempts to describe the macroscopic properties of device grade $a-\mathrm{Si}: \mathrm{H}$ within the framework of unifying microscopic structural models namely, (i) the negative correlation energy (U) model $U$ being the effective energy required to place a second electron in a singly occupied defect level, (ii) the thermal equilibration model and (iii) the dangling-band conversion model all of which rely on the hypothesis that the dominant structural defect in $a-\mathrm{Si}: \mathrm{H}$ is definitely the $\mathrm{Si}$ dangling bond.

Pantelides (1988) has proposed a floating bond model in which 'floating bond' is likely to be more mobile than the dangling bond. $\mathrm{He}$ argues that a mobile $\mathrm{D}$ centre is essential in accounting for the diverse and fascinating phenomena observed in $a-\mathrm{Si}$. The crystalline analog of a floating band is an Si interstitial, while that of a dangling bond is a $\mathrm{Si}$ vacancy. The floating bond, $\mathrm{Si}_{(5)}^{0}$ is an over-coordinated $\mathrm{Si}$ atom as much as the

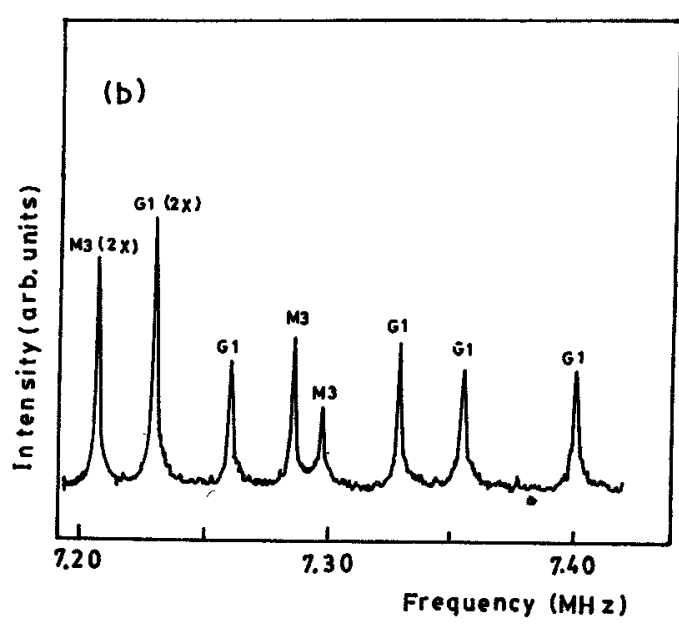

Figure 19. EPR and ENDOR spectra (at $23 \cdot 1244 \mathrm{GHz}$ ) of $\mathrm{Cr}^{+}$interstitial in Si-crystal. a. ESR absorption peaks for magnetic field along [001] axis. The lines from left-to-right correspond to $M_{\mathrm{s}}=-1 / 2 \leftrightarrow-3 / 2,5 / 2 \leftrightarrow 3 / 2, \leftrightarrow 1 / 2 \leftrightarrow-1 / 2,-3 / 2 \leftrightarrow 5 / 2$ and $+3 / 2 \leftrightarrow+1 / 2$ transitions of the $3 \mathrm{~d}^{5}$ system $S=5 / 2$, corresponding to labels $4,1,3,5$ and 2 of figure 5 and $\mathbf{b}$. ENDOR spectrum at a magnetic field of $826.830 \mathrm{mT}$, parallel to [110] at a microwave frequency of $23.1239 \mathrm{GHz}$. $G_{1}$ and $M_{3}$ correspond to 12 atom shells in which $\mathrm{Si}$ is in a glide plane and minor plane symmetry respectively. The peaks arise from NMR transitions between states $|-1 / 2 \leftrightarrow+1 / 2\rangle$ and $|-1 / 2 \leftrightarrow-1 / 2\rangle$ observed on $+1 / 2 \leftrightarrow-1 / 2$ EPR transition. 
dangling bond $\mathrm{Si}_{(3)}^{0}$ is an under-coordinated $\mathrm{Si}$ atom, and is characterized by a molecular orbital vis-a-vis the atomic orbital $\left(\mathrm{Si}-\mathrm{Sp}^{3}\right)$ of the dangling bond. The advantage with the floating bond is that 'bond switching' rather than 'bond breaking' results in high mobility needed for electrical transport. Self annihilation of the bond is possible and a 'kick-out' mechanism of diffusion of hydrogen is postulated wherein the stationary $\mathrm{Si}-\mathrm{H}$ bond is attacked by the mobile migrating floating bond, releasing or 'kicking out' a $\mathrm{H}$ into an interstitial. In this picture, the doping phenomenon in a semiconductor is conceived as a change in the coordination mediated by a mobile intrinsic defect, without the need for any electronic transition. Thus a 3-coordinated $P$ defect would combine with a floating bond to yield a $P_{4} . P_{4}^{0}$ or the neutral donor observed in the EPR, if P-doped $a-\mathrm{Si}: \mathrm{H}$ and $a-\mathrm{Ge}: \mathrm{H}$ through well resolved ${ }^{3 i} \mathrm{P}$ and ${ }^{75}$ As hyperfine structures provides some support for the floating bond model, although the question whether $D$ centres are mobile, still needs to be answered comprehensively. This pioneering ESR study of Stutzmann and Street (1985) has shown further that disorder-induced localization of states in $a-\mathrm{Si}: \mathrm{H}$ makes the donor hyperfine structure $\left({ }^{3 !} \mathrm{P}\right.$ or $\left.{ }^{75} \mathrm{As}\right)$ greater than that in crystalline $\mathrm{Si}$ and broadens the distribution of states with similar electronic character.

The observation by Stutzmann and Biegelsen (1989) of hyperfine structure ${ }^{24} \mathrm{Si}$ of the $g=2.0055$ ESR in undoped $a-\mathrm{Si}: \mathrm{H}$ containing the natural abundance of the ${ }^{29} \mathrm{Si}$ isotope (figure $17 \mathrm{~b}$, table 8 ) has allowed quantitative modelling of the underlying hyperfine and $g$ tensors. The structural parameters deduced for the $g=2.0055$ defect agrees very well with those of Si dangling bonds at the $\mathrm{Si} / \mathrm{SiO}_{2}$ interface. Significantly the ${ }^{29} \mathrm{Si}$ hyperfine structure observed by Stutzmann and Biegelsen (1989) appears to be incompatible with the floating bond model because the floating bond wave function would leave a much less weight on any $\mathrm{Si}$ atom than a dangling bond state.

ENDOR studies of $a-\mathrm{Si}: \mathrm{H}$ (made by reactive sputtering (RS) and glow-discharge (GD) decomposition) have shown (Yokomichi and Morigaki 1987; Morigaki 1988) the existence of at least two different types of dangling bonds: (i) normal dangling bonds and (ii) dangling bonds having a hydrogen as their neighbour. These experiments carried out at $7 \mathrm{~K}, 9.0 \mathrm{GHz}$ and rf current in the $1-150 \mathrm{~mA}$ show sample preparation dependent dangling bond configurations. While for RS samples, the ${ }^{29} \mathrm{Si}$ hyperfine splitting constants are $7.9 \mathrm{mT}$ and $3.6 \mathrm{mT}$ for type I and type II bonds mentioned above, they are $7.1 \mathrm{mT}$ and $3.8 \mathrm{mT}$ for GD samples. It is to be noted that ESR cannot distinguish between these two types of dangling bonds. The $g$-values are 2.0055 and 2.0052 for RS and GD samples respectively. From a local ENDOR study of ${ }^{1} \mathrm{H}$, in GD sample, an isotropic hyperfine interaction constant of $6 \mathrm{MHz}$ was deduced. The dangling bond densities estimated from ESR measurements are $1.4 \times 10^{18}$ and $1.6 \times 10^{18} \mathrm{~cm}^{-3}$ for GD and RS samples. Prolonged exposure $(\sim 5 \mathrm{~h})$ of $\mathrm{Gd}$ sample to IR for higher light from Xenon lamp increased the dangling bond density to $1.8 \times 10^{18} / \mathrm{cc}$.

There is a recent ENDOR evidence for clustered dangling bonds (Yokomichi and Morigaki 1993), by way of a rather large hyperfine splitting of $14.3 \mathrm{mT}$ due to ${ }^{29} \mathrm{Si}$ in unannealed and annealed $a-\mathrm{Si}: \mathrm{H}$ prepared at $70^{\circ} \mathrm{C}$ by glow discharge. This third type of dangling bond is more delocalized and has more $s$-character than those of isolated, normal dangling bonds.

8.2d Porous silicon: Crystalline silicon is an indirect band gap material, unlike the light emitting GaAs which is a direct band gap material. Porous silicon $(p-\mathrm{Si})$ is the result of efforts to 'engineer a direct band gap' in Si so that it could emit light like GaAs. Canham (1990) prepared this material by etching tiny 'worm holes' or pores into a Si wafer by applying hydrofluoric acid. The process is accelerated by a small voltage applied to the wafer. It is possible to etch away almost $80 \%$ of $\mathrm{Si}$, leaving an array of tiny 'dots' $\sim 50 \AA$ across, called 'quantum dots'. ESR studies on $p$-Si now made by the electrochemical anodization of Si wafers, have established (Kubo et al 1992; Yokomichi et al 1993) (figure 17c) that the main defect in porous silicon is a $\mathrm{Si}$ dangling bond located at the surface or at the $c-\mathrm{Si} / \mathrm{SiO}_{2}$ interface. It has trigonal symmetry with a principal axis along the (111) axis, with $g_{\| 1}=2.0022$ and $g_{\perp}=2.0078$. The anisotropy of the defect implies that the porous layer retains the crystallinity of the substrate. Illumination with $\mathrm{Xe}$ arc lamp light increases the intensity of the signals, which are partially recovered on thermal annealing (figure 17d), unlike in $a-\mathrm{Si}: \mathrm{H}$. This observation suggests that the light-emitting region of $p-\mathrm{Si}$ is mainly in the single crystal phase. This study has opened up both basic and applied lines of investigation including ENDOR studies of hyperfine interaction, to be compared to $c-\mathrm{Si}, a-\mathrm{Si}$ and $\mathrm{a}-\mathrm{Si}: \mathrm{H}$ as well as light-emitting characteristics both photo and electroluminescence.

\subsection{Germanium}

In contrast to the extensively studied case of $\mathrm{Si}$, the EPR study of $\mathrm{Ge}$ is rather restricted. As the study of electrons at dislocations in As-doped Ge (Pakulis and Jeffries 1981) has shown, at temperatures below $4 \mathrm{~K}$ the cylindrical sample itself becomes a microwave dielectric cavity with $Q \geq 10^{5}$, like $\mathrm{TiO}_{2}$, and improves the signal to noise ratio, making the electric EPR detection under optical excitation possible. 24 narrow $\left(\Delta H_{\mathrm{pp}}=1.4 \mathrm{mT}\right)$ lines attributed to well-defined distortions within dislocations and 4 broad lines $\left(\Delta H_{\mathrm{pp}}=4.0 \mathrm{mT}\right)$ due to As-donor 
hyperfine structure are observed. The $g$-tensor $g_{\|}=0.730 \pm 0.005, g_{\perp}=1.889 \pm 0.005$ and its angular dependence is assigned, by a careful correlation to the crystal structure of $\mathrm{Ge}$, to dislocation dangling bonds nearly perpendicular to the dislocation line, with a small but well-defined distortion $\left(1.2^{\circ}\right.$ tilt) in this dislocation.

$\mathrm{Li}$, a fast diffusing interstitial defect and a shallow donor in Ge gets trapped at low energy sites via acceptors, point defects and $\mathrm{O}^{-}$impurities. It is used in Li-precipitation technique to determine oxygen concentration in $\mathrm{Ge}$ crystals (the $\mathrm{Ge}: \mathrm{Li}$ detector). $\mathrm{Li}-\mathrm{O}$ is a paramagnetic molecular donor that has been studied at $\sim 24 \mathrm{GHz}$. The net-impurity concentration (donor: $\sim 10^{13} / \mathrm{cc}$, Oxygen $10^{14} /$ cc) of less than $10^{11} / \mathrm{cc}$ is insufficient to give an axially symmetric spectra $\left(g_{\|}=0.85 \pm 0.05, g_{\perp}=1.91 \pm 0.03\right)$ with the symmetry axis along $\langle 111\rangle$ (Haller and Falicov 1978). The studies on group $\mathrm{V}$ atom donors in $\mathrm{Ge}$ have been summarized by Ludwig and Woodbury (1962).

As with $a-\mathrm{Si}$, the most important defect in $a-\mathrm{Ge}$ is the dangling bond, characterized by a rather large $g$ of 2.02 due to considerable spin orbit coupling. Stutzmann et al (1987) have investigated $a-\mathrm{Ge}: \mathrm{H}$ containing $\mathrm{P}$ and As donors and detected donor dangling bonds $P_{4}^{0}$ and $\mathrm{As}_{4}^{\mathrm{0}}$.

\subsection{Oxidized silicon}

Thermal oxidation of $\mathrm{Si}$ wafer results in the formation of an $\mathrm{SiO}_{2}$ layer. The boundary between $\mathrm{Si}$ and $\mathrm{SiO}_{2}$ or the interlayer is conceptually a solid surface. Both $\mathrm{Si}$ and $\mathrm{SiO}_{2}$ are the constituent elements of the metaloxide semiconductor. Of special interest is the structure of defects in the $\mathrm{Si}-\mathrm{SiO}_{2}$ interlayer, as they determine the electrical and optical properties of the entire system. Three main ESR centres have been detected $P_{\mathrm{a}}, P_{\mathrm{b}}$ and $P_{\mathrm{c}}$ (Caplan et al 1977), of which $P_{\mathrm{b}}$ centre forms near the interface on (111) wafers. It has an axially symmetric $g$-tensor very similar to that of bulk $\mathrm{Si}$ defects having $\mathrm{Si}^{\mathrm{III}}$ bonded to $3 \mathrm{Si}$ atoms. The unpaired electron has a normal orientation to the (111) surface. This is unlike the commonly observed $E^{\prime}$ centre or the oxygen-vacancy defect of $\mathrm{SiO}_{2}$ (table 8).

The fundamental radiation-induced defect centres in synthetic fused silica free from impurities other than water, which forms the core of low-loss optical fibres and the gate oxide in Si-based MOS transistors are: the $E^{\prime}$ centre $(\equiv \mathrm{Si})$, the nonbridging oxygen hole centre $(\equiv \mathrm{Si}-\mathrm{O})$, and the superoxide radical $(\equiv \mathrm{Si}-\mathrm{O}-\mathrm{O})$. Variants of the $E^{\prime}$ centre $-E_{\alpha}^{\prime}, E_{\gamma}^{\prime}, E_{\jmath}^{\prime}$ have been observed.

Separation by implantation of oxygen (SIMOX) is an important manufacturing technique for industrial production of silicon-on-insulator substrates for the development of high-speed devices. SIMOX material is fabricated by implanting a high dose of high energy $\mathrm{O}^{+}$ ions into a $c-S i$ wafer which is subsequently annealed at a temperature near the melting point of $\mathrm{Si}$ $\left(T_{\mathrm{m}} \simeq 1412^{\circ} \mathrm{C}\right.$ ), when a $c-S i$ film well isolated from the $c-\mathrm{Si}$ substrate by a buried $a-\mathrm{SiO}_{2}$ (BOX) layer is formed. A K-band EPR study (Vanheusden and Stesmans 1993) of the buried $a-\mathrm{SiO}_{2}$ layer has detected the rarely observed $E_{\delta}^{\prime}$ component with $g_{1}=2.00168, g_{2}=2.00209$ and $g_{3}=2.00209$ making up about $20 \%$ of the total $E^{\prime}$ signal. This is a delocalized variant of $E^{\prime}$ centre, absent in reoxidized ' $\mathrm{BOX}$ ' or conventional thermal oxide, partially formed from small Si clusters in the BOX, implying the presence of excess $\mathrm{Si}$ near the BOX/substrate interface. This seems to be a good candidate for ESR imaging.

\subsection{Compound semiconductors}

Compared to the more ionic Si the ESR technique is less readily applicable to the increasingly covalent III-V semiconductors because (i) the large nuclear moments of ${ }^{27} \mathrm{Al},{ }^{71} \mathrm{Ga},{ }^{115} \mathrm{In},{ }^{31} \mathrm{P}$ and ${ }^{75} \mathrm{As}$ lead to large $(5-15 \mathrm{mT})$ line widths compared to $a<1 \mathrm{mT}$ for $\mathrm{Si}$ and (ii) the $3 \mathrm{~d}$ transition metals are sparingly soluble $\left(\leq 10^{17} / \mathrm{cc}\right)$ in these materials. Despite these limitations paramagnetic defects down to $10^{14} / \mathrm{cc}$ have been detected.

The most common intrinsic defects in these components besides vacancies and interstitials are the 'antisite' defects (e.g. $\mathrm{As}_{\mathrm{Ga}}$ in $\mathrm{GaAs}$ ) which are formed when anions occupy cations/anions, a situation arising from a rather small electronegativity differences between elements of groups III and V. The semi-insulating properties of melt-grown GaAs arise from an As-antisite related defect, EL2. Among the extrinsic defects are the 3d transition (and post transition) elements that substitute the cation and form deep acceptor states in general, their valency being determined by the position of the Fermi level in the band gap.

The information obtained from EPR (and ENDOR) studies of 3d-transition elements in III-V compounds, especially GaAs, InSb and $\mathrm{GaP}$ are, in general: (i) the local symmetry and position occupied by the transition metal atoms; (ii) the localization radius of wave functions of the impurity atoms of the $\mathrm{Fe}$ group, from a quantitative interpretation of ENDOR spectra; and in particular (iii) the magnitude of splitting of electronic energy levels of the transition metal ion by crystalline electric fields; (iv) identification of different nuclear species by their nuclear magnetic moments, and (v) the charge state of impurity.

8.5a III-V compounds: Apart from the impurity ESR (table 8), resonance from electrons in the conduction band has been observed in GaAs and InSb. The conduction electrons in these semiconductors are band electrons (unlike the free electrons in a metal) and possess very low effective mass $m^{*}\left(<0.1 \mathrm{~m}_{0}\right.$ the free electron mass). Thus their effective magnetic moments (Roth et al 1959) are given by 


$$
\mu_{\mathrm{eff}}=\mu_{B}\left[1-\frac{\left(\frac{m_{0}}{m^{*}}-1\right) \Delta}{3 E_{\mathrm{g}}+\Delta}\right],
$$

where $\mu_{\mathrm{B}}$ is the Bohr magneton, $\Delta$ the spin-orbit interaction and $E_{\mathrm{g}}$ the band gap and the $g$-values are very much different from 2 . Indeed, $g$ - which depends on concentration of electrons in the conduction band - is too low for GaAs $(0.28-0.56)$ while it is too high (50.7-48.8) for InSb. In the latter compound, the ESR line broadens with increasing electron concentration.

High-dose ion implantation of GaP crystals by $200 \mathrm{KeV}$ nitrogen ions at room temperature produces highly disordered layers therein, and, dangling bonds created thereby give an isotropic, $0.6 \mathrm{mT}$ wide symmetric Lorentzian ESR with $g=2.0032 \pm 0.0004$. This is apparently a very sensitive nondestructive method to determine the nature of damage produced by ion implantation, particularly because (i) the density of dangling bonds is proportional to the amount of disorder and (ii) the ESR spectral intensity is proportional to the number of defects produced by implantation.

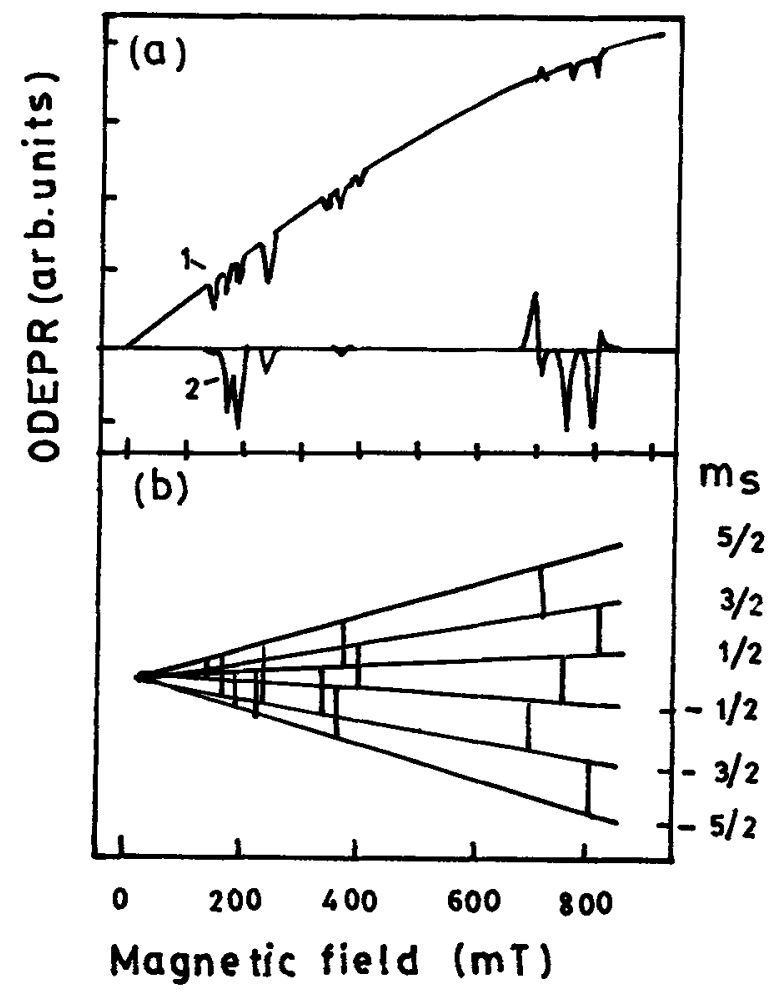

Figure 20. Optically detected K-band $(24 \mathrm{KHz})$ EPR spectrum of $\mathrm{Fe}^{3+}\left(3 \mathrm{~d}^{5}, S=5 / 2\right)$ in InP crystal at $1.5 \mathrm{~K}$, with dc magnetic field along [100] axis of the crystal. a. The spectra were measured (curve 1) at $4.3 \mathrm{eV}$, as the total change in the polarization of the ground state spin. The calculated spectrum is shown as curve 2 and $\mathbf{b}$. energy levels and EPR transitions $\Delta M_{\mathrm{s}}= \pm 1$, and $\Delta M_{\mathrm{s}}= \pm 2$ (forbidden in normal EPR) (Koschnick et al 1993).
$\mathrm{Fe}^{3+}$ defect in InP - a material of interest in integrated optical devices - has been investigated by EPR and optically detected EPR (ODEPR) (Koschnick et al 1993). This paramagnetic $\left(S=5 / 23 d^{5}\right)$ impurity - a deep acceptor - makes InP a semi-insulating material. Although ODEPR and EPR give the same $g$-factor and the same five fine-structure split lines (figure 20) with $g=2.024$ and $a$, and the cubic field splitting of 663 $\mathrm{MHz}$ and the lowest field fine structure transitions have opposite sign compared to others - a feature not observed in the ODEPR spectra of $\mathrm{Fe}^{3+}$ in GaAs. This anomalous behaviour arises from spin-lattice relaxations within $\mathrm{Fe}^{3+}$ Zeeman levels which operate faster for forbidden $\left(\Delta M_{\mathrm{s}}= \pm 2\right)$ transitions than for allowed $\left(\Delta M_{\mathrm{s}}= \pm 1\right)$ transitions. The spin-lattice relaxation times for the two transitions are $0.9 \mathrm{~ms}$ and $2.6 \mathrm{~ms}$, respectively.

Semi-insulating, undoped GaAs epitaxial layers grown by low temperature $\left(200^{\circ} \mathrm{C}\right)$ molecular beam epitaxy on $\mathrm{GaAs}$ or $\mathrm{Si}$ substrates have been exploited in electronic devices, photoconductive devices and integrated circuits as buffer layer in GaAs FETs and as gate insulating layer in MOSFETs. This material, typically a $5 \mu \mathrm{m}$ thick layer of $\mathrm{Ga}_{0.495} \mathrm{As}_{0.505}$, gives a thermal equilibrium EPR spectrum (Von Bardeleben et al 1992) with $g=2.04 \pm 0.01$ and $A(866 \pm 10) \times 10^{-4} \mathrm{~cm}^{-1}$ to be compared with $A=(890 \pm 10) \times 10^{-4} \mathrm{~cm}^{-1}$ obtained for one of the EL2-related antisite defects. The value of $A$ changes with thermal annealing. The lower value of $A$ is attributed to a change in the configuration of $\mathrm{As}_{\mathrm{Ga}}$ complex. These defects, which occur in both Czochralski- and Bridgmangrown GaAs, and in amorphous-MBE-grown GaAs, can be formed by electron irradiation. They produce distinct $A$ values, fingerprints for specific defect configurations.

8.5b II-IV compounds: Among the II-IV compounds, $\mathrm{CdTe}$ is well investigated for EPR of transition metals, shallow and deep donors. However, $\mathrm{Hg}_{1-x} \mathrm{Cd}_{x} \mathrm{Te}$ produced by implantation with B ions are of interest (Bowman et al 1987) in the fabrication of infrared photodiodes. The EPR spectra of $\mathrm{Hg}_{0.7} \mathrm{Cd}_{0.3} \mathrm{Te}$ and CdTe single crystals detected at $<78 \mathrm{~K}$ are sharp and isotropic, for implant doses of $\geq 1 \times 10^{16} \mathrm{mg} / \mathrm{cm}^{2}$. For identical implant conditions, $\mathrm{Hg}_{0.7} \mathrm{Cd}_{0.3} \mathrm{Te}$ gives near-intense and narrow signals than CdTe. Generally, the $g$-factors for all the compositions are identical and correspond to those for dangling bond defects in ion-implanted $\mathrm{Si}(g=2.0059)$ and $\mathrm{GaP}$ $(g=2.0032)$. While in chemically doped CdTe, EPR shallow donors $(g \sim 1.7)$ is found. The EPR intensities of $\mathrm{Hg}_{1-x} \mathrm{Cd}_{x} \mathrm{Te}$ are proportional to temperature and are thus due to isolated unpaired spins. The spectra above $6 \mathrm{~K}$ are symmetric and nearly Lorentzian and $\Delta H_{\mathrm{pp}}$ decreases from 0.17 to $0.12 \mathrm{mT}$ as the temperature increases to $30 \mathrm{~K}$, which could be due to thermally activated hops of paramagnetic spins between inequivalent locations. 
A novel technique (Rodriguez 1987) called electric dipole spin resonance has been applied to study spin-orbit coupling strength in the conduction band of zinc blende and wurtzite semiconductors - InSb and $\mathrm{Cd}_{1-x \mathrm{x}} \mathrm{Mn}_{x} \mathrm{Se}$ in particular. A parity violating spin-orbit coupling mixes states with opposite spin orientations and parities. This mixing allows electric dipole transitions between states which, in the absence of spin-orbit coupling, have equal parity and opposite spins. This interaction is given by

$$
\mu \cdot H=1 / 2 g_{0} \mu_{\mathrm{B}} \sqrt{\varepsilon} \mathcal{E}(\hat{n} \times \hat{\varepsilon}) \cdot \sigma,
$$

where $g_{0}$ is the $g$ factor of electrons for small fields $H_{0}, \mathcal{E}$ the $\varepsilon E$, electric field of the wave, $\varepsilon$ the dielectric constant of the material and $\sigma$ the Pauli spin matrix.

For InSb, $g_{\mathrm{H}}-g_{1}=-0.428$ where $g_{1}$ is the orbital $g$-factor which agrees very well with the theoretical value of -0.42 .

\subsection{Synthetic diamond}

Synthetic diamond (carbon) $\left(\geq 1.0 \mathrm{~cm}^{3}\right)$ single crystal, grown at a high temperature and under high pressure, has application in electronic devices. Diamond, which lies directly above silicon in the periodic table, is isostructural with silicon. But unlike $\mathrm{Si}$, it can admit only $\mathrm{Ni}$ among the $3 \mathrm{~d}$-group metals, which gives an ESR line at $g=2.0310 \pm 0.0005$ in polycrystalline form grown from a metal melt containing $\mathrm{Ni}$ (Loubser and Van Ryneweld 1966). This signal is isotropic down to $4 \mathrm{~K}$, suggesting a high symmetry site for $\mathrm{Ni}$ in the diamond lattice without any charge compensation.
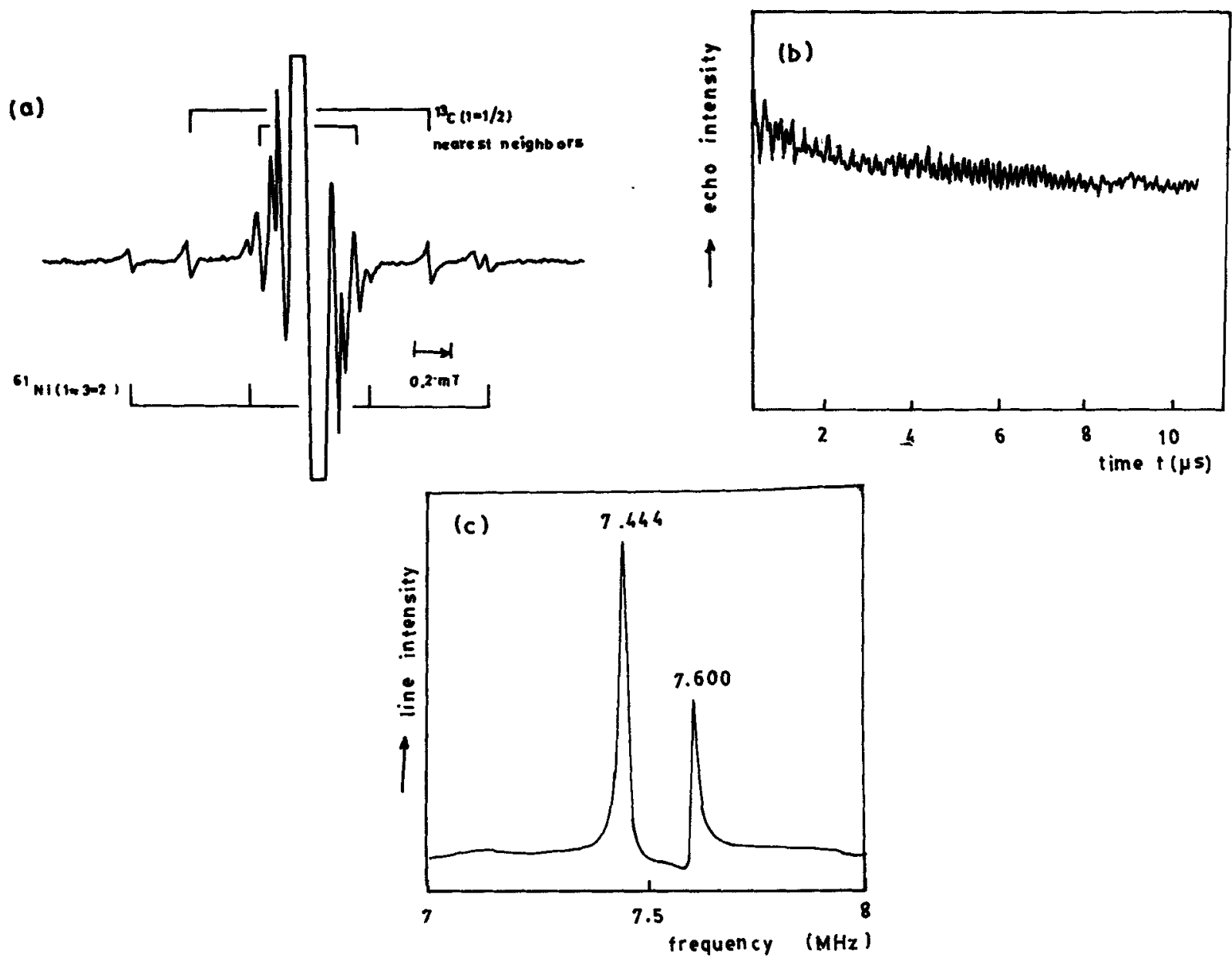

Figure 21. Continuous wave and Fourier transition EPR spectra of nickel in synthetic diamond. a. Low power $(0.2 \mathrm{~mW}) \mathrm{CW}$ EPR at $77 \mathrm{~K}$ showing ${ }^{61} \mathrm{Ni}(I=3 / 2)$ and ${ }^{13} \mathrm{C}(I=1 / 2)$ hyperfine structure in natural abundance, when the dc magnetic field is parallel to [111] axis of the crystal, with a $100 \mathrm{KHz}$ modulation field of $0.01 \mathrm{mT}$, b. three-pulse electron spin-echo modulation (time domain spectrum) from ${ }^{13} \mathrm{C}$ coupled to $\mathrm{Ni}$ in a diamond sample doped with $10 \%$ ${ }^{13} \mathrm{C}$, with the magnetic field parallel to [111] axis at $45 \mathrm{~K}$, using $200 \mathrm{~W}$ pulsed microwaves of frequency $9.22 \mathrm{GHz}$. Spacing (fixed) between first and second pulses is $0.40 \mu \mathrm{s}$ while the spectrum itself consists of 512 points spaced 20 ns apart and c. a portion of the frequency domain spectrum obtained from spectrum (b), showing two lines from ${ }^{13} \mathrm{C}$ coupled to $\mathrm{Ni}$ in ${ }^{13} \mathrm{C}$-enriched diamond (Isoya et al 1990). 
A comprehensive pulsed, Fourier transform and continuous wave (CW) EPR study has been carried out (Isoya et al 1990) on the $\mathrm{Ni}$ impurity in synthetic diamond, characterized by the $g=2.0319$ signal, whose linewidth is temperature dependent above $150 \mathrm{~K}$ due to strong spin-lattice relaxation. Figure 21 shows the $\mathrm{CW}$ spectrum, three-pulse echo modulation (In the 3-pulse echo technique, unlike in the 2-pulse spin echo technique discussed earlier, a third pulse applied whose distance from the first pulse is varied while the distance between first and second is fixed), and frequency domain spectrum for Ni. CW spectra (at $77 \mathrm{~K}$ ) give an isotropic hyperfine splitting $A\left({ }^{61} \mathrm{Ni}, I=3 / 2\right)$ of $0.65 \mathrm{mT}$ and two sets of nearly isotropic splittings due to hyperfine interaction with nearest and next-nearest neighbours ${ }^{13} \mathrm{C}$ atoms (the substitutional site in diamond has 12 'next-nearest' neighbours whereas the interstitial site has only six next-nearest neighbours). The CW EPR fails to resolve all the sets of super hyperfine lines due to interaction of unpaired electron of $\mathrm{Ni}$ with ${ }^{13} \mathrm{C}$ nuclei, which produce a 'modulation' of the spin echo which is detected in the timedomain. Accurate measurement of the super hyperfine coupling constant is possible for recording the frequency domain spectrum in an experiment akin to ENDOR. The effective spin of Ni has been determined to be $S=3 / 2$ (i.e. $\mathrm{Ni}$ is in -1 state at a substitutional site of tetragonal symmetry) $3 d^{7}$ from (i) the nutation of the magnetization by the microwave magnetic field $\left(H_{1}\right)$ and (ii) ${ }^{13} \mathrm{C}$ nuclear frequencies. This pioneering study has opened up new possibilities for the characterization of synthetic diamonds.

In a recent study, the ESR of diamond-like nuclei produced in a Si surface layer by high-dose C-ion doping has been measured (Izumi et al 1993).

The distribution of substitutional nitrogen centres (with resolved hyperfine structural from ${ }^{14} \mathrm{~N}$ ) and $\mathrm{Ni}$ impurities has been determined by ESR imaging (Furusawa and Ikeya 1990) (see § 12).

\section{Polymeric materials}

\subsection{General considerations}

Polymers have been extensively investigated from the points of view of local structural confirmation, degradation, oxidation polymerization, grafting, and permeation of oxygen in cross-linked polymers. Paramagnetic fragment(s) with the unpaired spin on the central carbon are invariably produced by $\gamma$ - or X-irradiation and the corresponding ESR spectra are generally symmetric patterns with tell-tale intensity distributions that aid radical identification (Ranby and Rabek 1977). Chain-growthpolymerization - an important polymeric process occurs by free radical or ionic mechanism. The growth of a single polymer chain or a macro molecule is due to the propagation of one kinetic chain reaction. Every free radical chain reaction requires a separate initiation step in which a radical species is generated in the reaction mixture. Two processes are involved:

(1) Homolytic cleavage

$$
\mathrm{AB} \stackrel{+m^{\prime}}{\longrightarrow} \mathrm{A}+\mathrm{B} .
$$

(2) Unpaired electron transfer from initiator/to the monomer fragments formed by dissociation,

$$
\begin{aligned}
& \mathrm{R}-\mathrm{R} \stackrel{+m}{\longrightarrow} 2 \mathrm{R} . \\
& \mathrm{R} .+\mathrm{CH}_{2}=\mathrm{CHX} \rightarrow \mathrm{R}-\mathrm{CH}_{2}-\dot{\mathrm{C}} \mathrm{HX},
\end{aligned}
$$

where $\mathrm{R}$ is an alkyl group and $\mathrm{X}=\mathrm{Cl}$ or $\mathrm{O}$ and the dots denote unpaired electrons.

An example of an ESR study of molecular motion is the tunneling of methyl groups (at $6 \mathrm{~K}$ ) in a radical formed by $\gamma$-irradiation of polymethyl methoacrylate (Iwasaki et al 1971). Other radicals trapped at $77 \mathrm{~K}$ include $\mathrm{CH}_{3} \mathrm{OOC}^{\circ}$ or $\mathrm{HOOC}^{-}$which gives a singlet, $\mathrm{OHC}^{\cdot}$ which gives a doublet (spaced $13.0 \mathrm{mT}$ ) and of course ${ }^{\circ} \mathrm{CH}_{3}$ which gives a quartet with an intensity ratio $1: 3: 3: 1$ and a spacing $2.3 \mathrm{mT}$ arising from interaction of unpaired spin with three equivalent $\mathrm{H}$ nuclei. Generally the ESR spectrum of polymer subjected to irradiation may be characterized by the equation

$$
\mathrm{H}_{\mathrm{res}}=\frac{h v}{g \beta}-\sum_{i=1}^{\infty}\left(A_{\mathrm{i}}+B_{\mathrm{i}}\right) m I_{\mathrm{i}},
$$

where $A_{\mathrm{i}}$ is the isotropic hyperfine interaction between the radical and nucleus $i$, and, $B_{i}$ the anisotropic part of this interaction depending on the orientation of the radical. The singlet ESR referred to above arises when $A=B=0$. For most hydrocarbon radicals $B \neq 0$ only for $\alpha$-protons i.e. $\mathrm{H}$ nuclei directly bonded to radical $\mathrm{C}$ atom. In highly-oriented polymers and single-crystal polymers these radicals have a strongly orientation-dependent hyperfine structure. In general, for $n$ equivalent protons a spectrum with $(n+1)$ lines whose intensities are as the coefficients of binomial distribution $(1+x)^{\mathrm{n}}$ is observed. Thus for ${ }^{-} \mathrm{CH}_{3}(n=3), 4$ lines result.

Highly-oriented polyethylene monofilaments when irradiated by fast electrons yield the radical

$$
\underset{\beta}{-\underset{\mathrm{CH}_{2}}{\mathrm{C}}-\underset{\mathrm{C}}{\mathrm{C}} \mathrm{H}-\mathrm{CH}_{2}-}
$$

when the fibre axis is parallel to the magnetic field, the $\alpha$ and four equivalent $\beta$ protons have the same hyperfine splitting so that five equivalent protons give a spectrum with an intensity distribution $1: 5: 10: 10: 5: 1$. But when the fibre axis is normal to the magnetic field, $\alpha$ 
and $\beta$ protons have different hyperfine splittings so that a quintet of lines due to four equivalent $\beta$ protons, with each quintet split into a doublet by the $\alpha$ proton are observed. The intensity ratio of the overall spectrum is $1: 1: 4: 4: 6: 6: 4: 4: 1: 1$. The primary radicals produced by irradiation or milling are usually detected at low temperatures in most of the common degraded polymers. Table 9 presents the radicals thus produced in selected polymers. An exhaustive list is given by Kausch (1978). ESR affords a direct study of bond rupture (and formation of 'mechano radicals') in highly oriented fibres and films of polyethylene, propylene, PTFE, PMMA, polystyrene, polyesters and polynucleotides. The fracture of rubber has also been studied (Ranby and Rabek 1977). Free radicals formed and trapped in polymer matrices under certain conditions can initiate graft polymerization. The grafting reaction depends on (i) the physical state of the polymer, and (ii) the properties of free radicals formed in the polymer. A sample of electron or $\gamma$-irradiated polymer brought into contact with a monomer can diffuse into the polymer phase and thus reach the trapped radical site. The case of butadiene diffusion in polyvinyl chloride has been studied (Hamanone et al 1974). The use of a 'dynamic spin probe' (e.g. the nitroxide $\mathrm{O}=\mathrm{N}-\mathrm{O}$. dissolved in benzene) has helped probe glass transition behaviour of polymers, chain motions on the surfaces of as-grown and annealed polyethylene single crystals in relation to surface structure, and polymer-plasticizer interaction in plasticized PVC. Among other ESR applications are the donor-acceptor polymer complexes and the reinforcing properties of carbon black in rubber vulcanization.
Recent applications include studies of (i) radiationinduced cross linking of polyethylene in the presence of acetylene, (ii) molecular motion of chain-end radicals of polyethylene molecules anchored on fresh surfaces of polyethylene and PTFE, (iii) interaction of mechanicallygenerated polypropylene radicals with phenolic antioxidant in the presence of oxygen, (iv) thermallygenerated free radicals in PMR-15 polyimide resins, (v) solitons and phase transitions in conducting polymers, and (vi) photo-induced charge transfer from polymer to $\mathrm{C}_{60}$ superconductor.

Some of these applications are discussed in detail below.

\subsection{Mechano-radicals}

9.2a Polymerization: Mechano-radicals are produced by mechanical fracture (e.g. grinding) of polymers at low temperatures. The radicals known as chain-end type free-radicals are produced by the 'homolytic bond scission' of carbon-carbon bond in the polymer main chain. For e.g. a polymer $P$ with a $P-P$ bond undergoes the scission:

$$
P-P \rightarrow P^{*}
$$

to yield the mechano-radical $P^{*}$. In addition, 'mechanoanions' produced by heterolytic bond scission

$$
P-P \rightarrow \underset{\substack{\text { mechano } \\ \text { anion }}}{P^{-}}+\underset{\substack{\text { mechano } \\ \text { cation }}}{P^{+}}
$$

\begin{tabular}{|c|c|c|}
\hline Polymer & $\begin{array}{l}\text { Experimental } \\
\text { conditions }\end{array}$ & Radical(s) found \\
\hline 1. Polyethylene & $\begin{array}{l}\text { Milling } \\
(77,240 \mathrm{~K})\end{array}$ & $-\mathrm{CH}_{2}-\dot{\mathrm{C}} \mathrm{H}_{2}$ \\
\hline 2. Polypropylene & Milling & $-\mathrm{CH}_{2}-\mathrm{CH}_{3} \dot{\mathrm{C}} \mathrm{H}$ \\
\hline 3. Polystyrene & $\begin{array}{l}\text { Milling } \\
(77 \mathrm{~K})\end{array}$ & $-\mathrm{CH}_{2}-\mathrm{C}_{6} \mathrm{H}_{5} \dot{\mathrm{C}} \mathrm{H}$ \\
\hline 4. PTFE & Ball mill & $-\mathrm{CF}_{2}-\dot{\mathrm{C}}_{2}$ \\
\hline 5. Polyvinyl alcohol & $\begin{array}{l}\text { Grinding } \\
(80-170 \mathrm{~K})\end{array}$ & $\begin{array}{l}\mathrm{CH}_{2}-\mathrm{OH} \dot{\mathrm{C}} \mathrm{H} ; \\
-\mathrm{OHCH}-\dot{\mathrm{C}} \mathrm{H}_{2}\end{array}$ \\
\hline 6. Polymethyl methacrylate & $\begin{array}{l}\text { Milling } \\
(300 \mathrm{~K}, 77 \mathrm{~K})\end{array}$ & $-\mathrm{CH}_{2}-\left(\mathrm{COOCH}_{3}\right) \mathrm{CH}_{3} \dot{\mathrm{C}}$ \\
\hline 7. Polycarbonate & $77 \mathrm{~K}$ & $-\mathrm{O}-\dot{\mathrm{C}}_{6} \mathrm{H}_{4} ;-\mathrm{OC}-\mathrm{H}_{4} \dot{\mathrm{C}}_{6}$ \\
\hline 8. Polyethylene oxide & $\begin{array}{l}\text { Grinding } \\
(80-230 \mathrm{~K})\end{array}$ & $-\mathrm{O}-\dot{\mathrm{C}} \mathrm{H}_{2}$ or $-\mathrm{O}-\dot{\mathrm{C}} \mathrm{H}$ \\
\hline 9. Natural silk & $\begin{array}{l}\text { Milling } \\
(77 \mathrm{~K}-120 \mathrm{~K})\end{array}$ & $\begin{array}{l}-\mathrm{NH}-\dot{\mathrm{C}} \mathrm{H}_{2} ;-\mathrm{CO}-\dot{\mathrm{C}} \mathrm{H}_{2} \\
-\mathrm{NH}-\mathrm{CH}-\mathrm{CH}_{3} ; \\
-\mathrm{CO}-\dot{\mathrm{C}} \mathrm{H}-\mathrm{CH}_{3}\end{array}$ \\
\hline
\end{tabular}

Table 9. Primary radicals in certain degraded polymers detected by ESR*t

*Usual conditions: vacuum or inert atmosphere.

${ }^{\dagger}$ Adapted from Kausch (1978). 
can be stabilized for instance by electron transfer due to contact in the dark e.g.

$$
P^{-}+M \rightarrow P^{*}+M^{\cdot} \text {, }
$$

(where $M=\mathrm{a}$ molecule with high electron affinity like tetracyano ethylene TCNE) so that $P^{*}$ may be detected by ESR spectroscopy. It is significant that these mechano radicals initiate polymerization at low temperatures under vacuum, as has been demonstrated for methyl methacrylate and ethylene (Sakaguchi et al 1989).

Polyvinylidenefluoride (PVDF) fractured alone and with isobutyl vinyl ether (IBVE) as well as TCNE (Sakaguchi et al 1988, 1989) by using a vibration ball mill (in the dark and at $77 \mathrm{~K}$ under vacuum) to produce dramatic changes in the ESR spectra (figure 22) show that (i) mechanical fracture of TVDF alone induces a heterocyclic bond scission in the main chain,

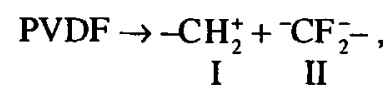

producing mechano cation $-\mathrm{CH}_{2}^{+}$and (ii) a cationic polymerization of IBVE is initiated by this mechano cation

$$
\begin{aligned}
-\mathrm{CH}_{2}^{+}+\mathrm{CH}_{2} & =\mathrm{CH}\left(\mathrm{OCH}_{2} \mathrm{CH}\left(\mathrm{CH}_{3}\right)_{2}\right) \rightarrow \\
& -\mathrm{CH}_{2}^{-}+{ }^{\cdot} \mathrm{CH}-{ }^{+} \mathrm{CH}\left(\mathrm{OCH}_{2} \mathrm{CH}\left(\mathrm{CH}_{3}\right)_{2}\right),
\end{aligned}
$$

eventually producing the PVIBE homopolymer through the propagation reaction.

$$
\begin{aligned}
& \cdot \mathrm{CH}_{2}-{ }^{+} \mathrm{CH}\left(\mathrm{OCH}_{2} \mathrm{CH}\left(\mathrm{CH}_{3}\right)_{2}\right) \rightarrow \\
& \mathrm{CH}_{2}^{-}-{ }^{+} \mathrm{CH}-{ }^{+} \mathrm{CH}\left(\mathrm{OCH}_{2} \mathrm{CH}\left(\mathrm{CH}_{3}\right)_{2}\right) .
\end{aligned}
$$

A superposition of the spectra of radicals I, II and III is seen in the ESR spectra (not shown), (iii) $\mathrm{TCNE}^{-}$is formed by the abstraction of electrons by TCNE from the anion that is produced by the heterogeneous bond scission of $\mathrm{C}-\mathrm{C}$ bonds in the PVDF main chain, via the following reactions.

$$
\begin{aligned}
& \text { PVDF } \rightarrow 2 R^{\cdot}, \\
& \text { PVDF } \rightarrow R^{-}+R^{+}, \\
& R^{-}+\mathrm{TCNE} \rightarrow R^{\cdot}+\mathrm{TCNE}^{-},
\end{aligned}
$$

(iv) photo irradiation of a fractured sample results in

$$
\mathrm{R}^{-}+\mathrm{TCNE} \stackrel{h \nu}{\longrightarrow} \mathrm{R}^{\cdot}+\mathrm{TCNE}^{-},
$$

and increase the yields of total radical mechanism.

9.2b Radical motion: Molecular motion of polymer chains seems to be restricted by the specific interaction of the chains with matrix molecules surrounding them and/or the intermolecular forces holding the chains in the matrix. The study of such motion is important in looking - for instance - at the miscibility of polymer blends (Shimada et al 1988). Sagakuchi et al (1993) have made a comprehensive ESR study of chain-end radical motion of polyethylene radicals anchored on fresh surfaces of polyethylene (PE) and polytetrafluoroethylene (PTFE). This radical, produced by mechanical fracture (under vacuum at $77 \mathrm{~K}$ ), can initiate radical polymerization of ethylene monomer and produce polyethylene chains which are bound to the molecules composing the fresh surface of the polymer. The mobility (free rotation) of this radical anchored on fresh PTFE surface is high even at $77 \mathrm{~K}$ because the weak intermolecular forces between radical and PTFE molecules cause the radical to protrude from the PTFE surface, the latter being an isolated system. But the same chain-end radical absorbed
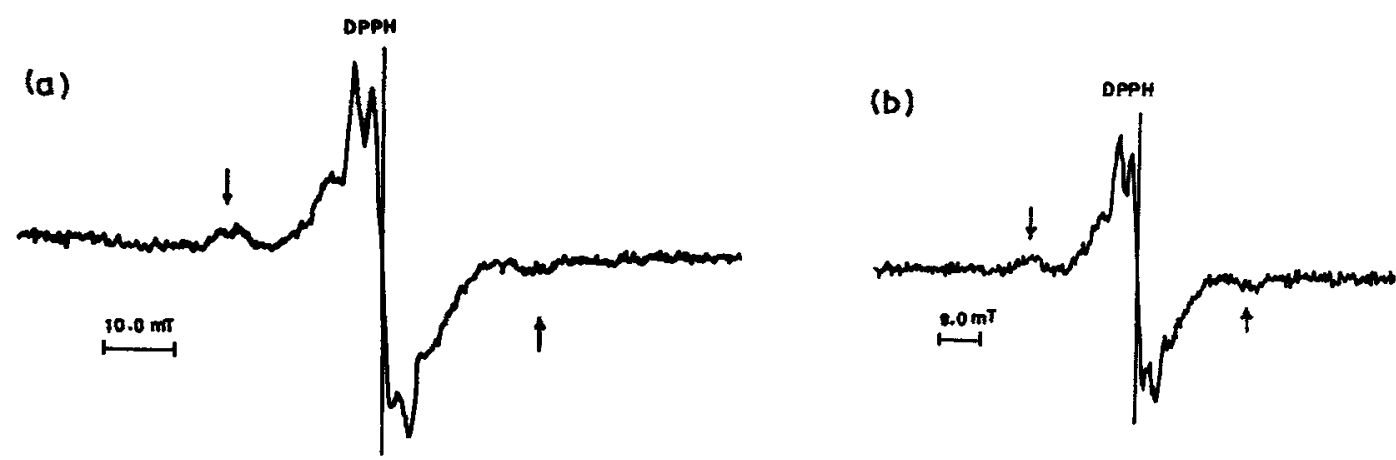

Figure 22. ESR spectrum from PVDF fractured (a) without IBVE, and (b) with IBVE, in the dark in a vacuum at $77 \mathrm{~K}$, at a microwave power level of (a) $0.4 \mathrm{~mW}$ and (b) $0.04 \mathrm{~mW}$ (DPPH was used as $g$-marker). Arrows indicate wing peaks with a hyperfine constant of $43.6 \mathrm{mT}$ due to $2 A_{\|}$from two $\alpha$-F atoms, attributed to $\mathrm{CF}_{2}$. The inner triplet with $1: 2: 1$ and is due to the mechano radical ${ }^{\circ} \mathrm{CH}_{2}^{-}$ (Sakaguchi et al 1989). 
on a fresh surface of PE is held more strongly to the $\mathrm{PE}$ molecule and exchange motion takes place in this nonisolated system at $77 \mathrm{~K}$. These conclusions are based on computer simulations of observed sextet and quintet spectra of radical for the case of PE and PTFE surfaces respectively. The simulation was done assuming Gaussian lineshape function and spin exchange rate.

9.2c Charge generation mechanism in polymers: Tribo-electricity in polymers arises when there is a transfer of charge between two insulating polymers or between a metal and a polymer when they are brought into contact and then separated. This phenomenon, which has applications in electrophotography and dry-ink electrophotography and is also the cause of troublesome static electricity, is a century-old problem (Harper 1964). In a recent quantitative model of contact electrification polymers (Duke and Fabysh 1978), it has been shown that steady-state-exchanged charge resides in intrinsic molecular ion states and the sign and order of magnitude of measured contact charge exchange between polystyrene and co-polymer of styrene and methyl methacrylate have been correctly predicted.

A novel charge generation mechanism for the reaction of mechano anions and mechano radicals produced by mechanical fracture of a polymer main chain, based on ESR studies has been reported in an attempt to deal with triboelectricity as a phenomenon involving the mechanical fracture of a polymer chain on the friction surface. By measuring the yield of the ESR active TCNE $^{-}$produced under milling of PMMA, PP, PE, PVDF and PTFE with TCNE in the dark at $77 \mathrm{~K}$, Sagakuchi et al (1990) have shown that (i) an electron transfer occurs from the mechano-anions to the mechano radicals produced by mechanical fracture of polymer main chains via friction: $A^{-}+B^{*} \rightarrow A^{*}+B^{-}$, where $A$ and $B$ are the two polymers involved, (ii) the sign of the charge induced by friction can be estimated from the electron release potential of $A$ and electron affinity of $B$ and (iii) the triboelectric series exist: PMMA - PP - PE - PVDF - PTFE, based on the reaction of $A^{-}$with TCNE, varying from high to low from left to right, a significant conclusion obtained. The ESR samples of photo-irradiated with filtered infrared radiation, are identical to the triboelectric series of polymers (Heniker 1962).

\subsection{Inclusion polymerization}

Inclusion polymerization is an important phenomenon in the synthesis and study of inclusion compounds. An ESR study of these compounds usually uses the polymer host matrix (example, perhydrotriphenylene, PHTP) as an environment in order to observe long-lived radicals of guest molecule. However, inclusion polymerization studies could also concentrate on (i) the nature of the active centres involved and (ii) the structure of the inclusion compounds. ESR could answer questions regarding the host-guest and guest-guest interactions at an intermediate level between the presence of monomers and the polymer.

Sozzani et al (1986) have sought to establish through a comparative study of a number of homogeneous monomers the structure of the propagating chain ends derived from diene monomers, (butadiene, 1,3-pentadiene, isoprene, 2,3-dimethyl betadiene, 2-methyl pentadiene and 2,4-hexadiene), the structure of the propagating chain ends derived from diene monomers, in particular, the prevailing direction of propagating monomer units and the strict relationship between the last and the preceding monomer. During polymerization, ESR spectra attributable to allyl-type propagating radicals, e.g.

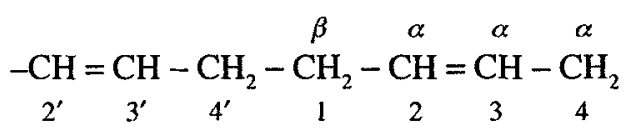

in polybutadiene, characterized by a 6-line spectrum, spaced $1.4 \mathrm{mT}$, and an intensity ratio $1: 5: 10: 5: 1$ (figure 23) were observed. Except for this radical in this polymer, all other radicals are stable over long periods of time, in the range $-150^{\circ}$ to $+60^{\circ} \mathrm{C}$. Thus, the included methyl polybutalines are confirmationally fixed.

The ESR spectrum of allyl radical in polybutadiene included in PHTP is temperature-dependent (figure 23) and rotates around the $C_{1}-C_{2}$ bond even at room temperature, i.e. it is mobile even at ambient temperature. The five $\alpha_{1}$ and $\beta \mathrm{H}$ nuclei of the above radical are coupled to the unpaired electron by about the same constant, while the $\alpha_{2}$ hydrogen is coupled to it by a much smaller coupling constant. The $\beta-\mathrm{H}$ constants are given by

$$
a_{\beta}=B^{0} \rho_{\pi} \cos ^{2} \theta,
$$

where $\theta$ is the angle between the $p_{z}$ orbital axis of the allyl system and the projection of the $C_{1}-H$ bond on the plane perpendicular to the $\mathrm{C}_{1}-\mathrm{C}_{2}$ bond, $B^{0}$ is a constant $(5.88 \mathrm{mT})$ and $\rho$ the spin density on the $\mathrm{C}_{2}$ carbon (assumed 0.58 for the allyl radical). The above equation can be solved by cases of the rotation around $\mathrm{C}_{1}-\mathrm{C}_{2}$ bond either frozen by the crystalline matrix or fast relative to the ESR time scale. A freely rotating $\mathrm{C}_{1}-\mathrm{C}_{2}$ bond would mean that both $\beta$ hydrogen nuclei must couple with the same constant, i.e. $\left\langle\cos ^{2} \theta\right\rangle=1 / 2$ and $a \beta=1 / 2 B^{0} \rho_{\pi}=1.7 \mathrm{mT}$. The observation of a temperature-dependent, reversible, ESR spectrum confirms this hypothesis. Thus rather wide chain movements near the chain end must be allowed inside the canal inclusion compound. 


\subsection{Undoped and doped polyacetylene}

Ever since Shirakawa and Ikeda (1971) prepared polycrystalline polyacetylene, $(\mathrm{CH})_{x}$, a conjugated polymer, there has been an explosion of literature in the so-called 'synthetic metals', as the highly conducting, quasi onedimensional polymeric materials are called. ESR has been a natural choice for the study of these systems in which intrinsic paramagnetic centres - to the extent of one spin per $\sim 1500$ carbon atoms - are present in trans- $(\mathrm{CH})_{x}$ with an order of magnitude less in cis- $(\mathrm{CH})_{x}$. While the spins are mobile in pure trans- $(\mathrm{CH})_{x}$, they are fixed in $c i s$-rich-(CH) $)_{x}$.

Undoped polyacetylene can be regarded as a Peierlsdistorted linear chain (Rice 1979) involving $\pi$ electrons at the half-filled band, with an energy of formation $E_{\pi}$ and length $l$, which may be related to $\pi$-electron energy gap, $2 \Delta$ and bandwidth, $W$. Numerical values of $E_{\pi}, \Delta$ and $W$ are calculated to be $0.4,10$ and $1 \mathrm{eV}$, respectively. Using a harmonic potential $1 / 2 a u^{2} \quad(u=$

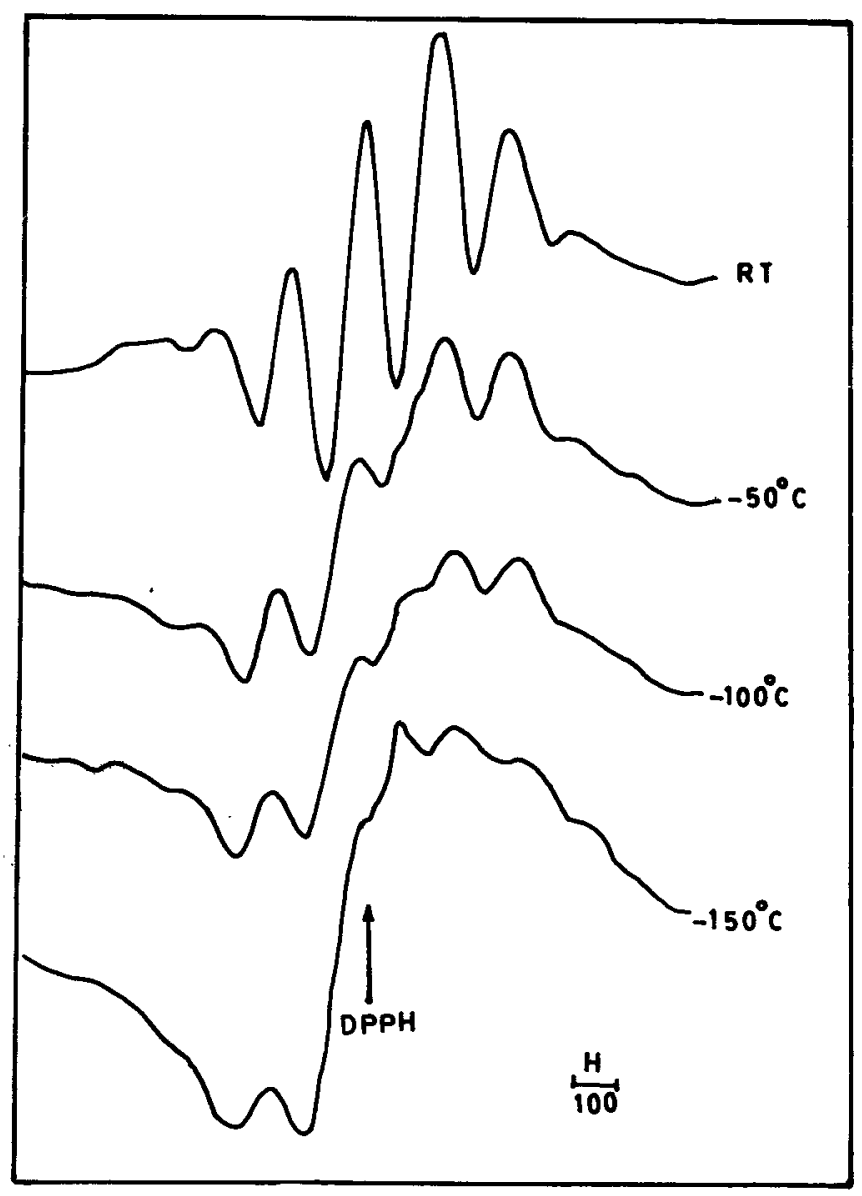

Figure 23. ESR spectrum of polybutadienyl radical included in PHTP as a function of temperature showing that the rotation around the $\mathrm{C}_{1}-\mathrm{C}_{2}$ single bond is mobile enough at nontemperature and that rather wide chain movements near the chain end must be allowed (Sozzani et al 1986). displacement) in which the electrons move, it has been shown that $l / a \approx W / \Delta$ so that $l / a \approx 10$. If polyacetylene is highly doped with electrons/holes then the latter would be accommodated in the form of charged $\pi$-phase kinks or soliton distortions rather than filling available $\pi$ electron band states. These distortions in the band alternation of the polyacetylene chain generate, gradually over a length $l$, a $180^{\circ}$ variation in the phase of the regular band alternation, separating energetically equivalent change segment in which single-double-bonds are shifted by one $\mathrm{C}-\mathrm{C}$ length. These distortions are like charged domain walls. Thus, while the undoped $(\mathrm{CH})_{x}$ would represent the dilute limit in which charges are stored in the form of spinless charged solitons, lightlydoped $(<6 \mathrm{~mol} \%)$ would create charged domain walls which move to conduct electricity. Heavy doping (e.g. with alkali metals, $\mathrm{AsF}_{5}$ etc.), would cause an abrupt, soliton to metal phase transition, which has been shown to be a first-order phase transition from in situ ESR studies of electrochemically Na-doped $(\mathrm{CH})_{x}$ in which the ESR linewidth exhibits a step-like increase, with hysteresis, as functions of chemical potentials.

A thorough analysis of the ESR lineshape and linewidth in undoped $(\mathrm{CH})_{x}$ (Holczer et al 1981; Nechtschien et al 1983), has established that the ESR linewidth is extremely sensitive to ambient contamination (which does not increase spin susceptibility) and is thus dependent on sample preparation. By taking extreme precautions at various stages of material preparation, they have obtained a $\Delta H_{\mathrm{pp}}$ of $0.044 \mathrm{mT}$, while a soft doping process (Chen et al 1985) has yielded a very narrow $\left(\Delta H_{\mathrm{pp}}=0.028 \mathrm{mT}\right)$ line. This narrow line, represents the diffusive (onedimensional) transport of mobile spins in trans- $(\mathrm{CH})_{x}$, whereas the fixed spins of cis- $(\mathrm{CH})_{x}$ would broaden the line considerably. The soliton makes fast transitions from trapped to diffusive states, giving rise to a single ESR line.

The first-order transition to the metallic state in polyacetylene, when it is heavily doped, has been explained (Kivelson and Heeger 1985) in terms of a strong coupling polymer model illustrated in figure 24 .

An abrupt crossover from a lattice of charged soliton to a regular array of polaron-like distortions accounts for this phase transition. In this model, doped $(\mathrm{CH})_{x}$ is a 'dirty metal' in which there is a disorder-induced gradual evolution of finite density of states in the Peierls gap. While the mid-gap-band is full in the soliton lattice (figure 24a) the upper polaron band, (close to the conduction band) is half full in the polaron lattice (figure 24b).

\subsection{Stretched polymers}

Polymeric materials often form radicals when under stresses of various types and these may live long enough 
in the solid to give strong ESR signals. For instance, stretching of nylon under vacuum, directly in the ESR cavity, well-defined, structured spectra, similar to those obtained upon $\gamma$-irradiation are obtained (Campbell and Peterlin 1968). In conducting polymers, such as 'new' polyacetylene (prepared by nonsolvent polymerization linked with thermal treatment of catalyst solution at higher temperature), stretching (say by a factor of five) is an important means of increasing the probability of charge carrier hopping and enhancing electrical conductivity by 5-6 orders of magnitude, because it is through stretching that a parallel arrangement of polyacetylene chains is achieved. The basic question in conducting polymer research is: What molecular and morphological structure produces a high conductivity $\left(\sim 10^{5} \mathrm{~S} \mathrm{~cm}^{-1}\right)$ upon doping? In highly oriented polymers the ESR linewidth shows a dependence on the angle $\Theta$ between the direction of orientation of the polymer chains in the sample and the dc magnetic field of the ESR spectrometer. Thus, the degree of stretching is easily correlated with molecular property of the polyacetylene chain (Bartl et al 1993). The degree of stretching $d_{\mathrm{s}}=l / l_{0}(l=$ length after stretching, $l_{0}=$ initial length) while the ESR linewidth-related diameter $\delta=\left(\Delta H_{\|} \cdot \Delta H_{\perp}\right) / \Delta H_{\|}\left(\Delta H_{\|}\right.$and $\Delta H_{\perp}$ are linewidth for $\Theta=0$ and $90^{\circ}$, respectively), while $d_{\mathrm{s}}$ changes from 1 to $7, \delta$ varies from 0.22 to 0.34 . $\delta$ increases drastically at low degree of stretching, while at high orientation, $\delta$ shows a small effect. These behaviours correlate with the increase of electrical conductivity as a function of stretching.

The 'probes' for these studies, of structural and orientational aspects are the so-called neutral solitons which are paramagnetic species with spin but without charge (Bartl et al 1992). The mobile spins move along the conjugated chain segments obtained by 'doping' to produce motionally narrowed ESR lines through exchange interaction of electron spins with proton spins. Thus the length of the undisturbed conjugate chain segments determines the ESR linewidth. Short segments show broad ESR lines while long segments produce narrow ESR lines. As already stated, ESR linewidth shows a characteristic dependence with respect to the orientation of the polymer chain relative to the magnetic field.

\subsection{Polymer-fullerene charge transfer}

Photo-induced electron transfer is of fundamental interest in the photophysics and photochemistry of excited states in organic molecules as a synthetic approach to an understanding of solar energy conversion and for applications in molecular information storage. Indeed, donorbridge acceptor type 'super molecules' have been proposed as bistable information storage entities (Carter 1987). An interesting case of photo-induced electron transfer from the polymer poly[2-methoxy,5-(2' ethylhexyloxy)-P-phenylene vinylene] (MEH-PPV) onto the carbon superconductor or Buckminsterfullerene $\left(\mathrm{C}_{60}\right)$ has been investigated (Sariffici 1992). In this light-induced ESR or LESR experiment, ion radicals originating from charge separation

$$
D+A \rightarrow D^{+\cdot}+A^{-\cdot},
$$

where $D$ is the electron donor (MEH-PPV) and $A$ the electron acceptor $\left(\mathrm{C}_{60}\right)$, are photo-generated and detected in situ. Upon irradiation at $80 \mathrm{~K}$ with an argon-ion laser beam $(2 \cdot 14 \mathrm{eV}: 150 \mathrm{~mW})$ two photo-induced signals, (i) at $g \sim 2.0000$ with $\Delta H_{\mathrm{pp}}=0.72 \mathrm{mT}$, and (ii) at $g$ $\sim 1.9955$ with $\Delta H_{\mathrm{pp}}=0.6 \mathrm{mT}$, due to (MEH-PPV) ${ }^{+}$and $\mathrm{C}_{60}^{-}$were observed (figure 25 ). At $80 \mathrm{~K}$, the ESR signal intensity increases during light $\mathrm{ON}-\mathrm{OFF}-\mathrm{ON}-\mathrm{OFF}$ cycles, indicating the accumulation of photo-generated spins associated with $D^{+\cdot}$ and $A^{-\bullet}$ species. At $200 \mathrm{~K}$ the LESR signal nearly vanishes, demonstrating the reversibility of the process, ruling out any residual spin due to photo chemical reaction products. The projected applications of this prototypical reaction are in molecular optoelectronics, nonlinear optics and photovoltaics.

\subsection{Polymer imaging}

The fact that ESR signals can be enhanced and controlled by adding dopants to polymers has been exploited in

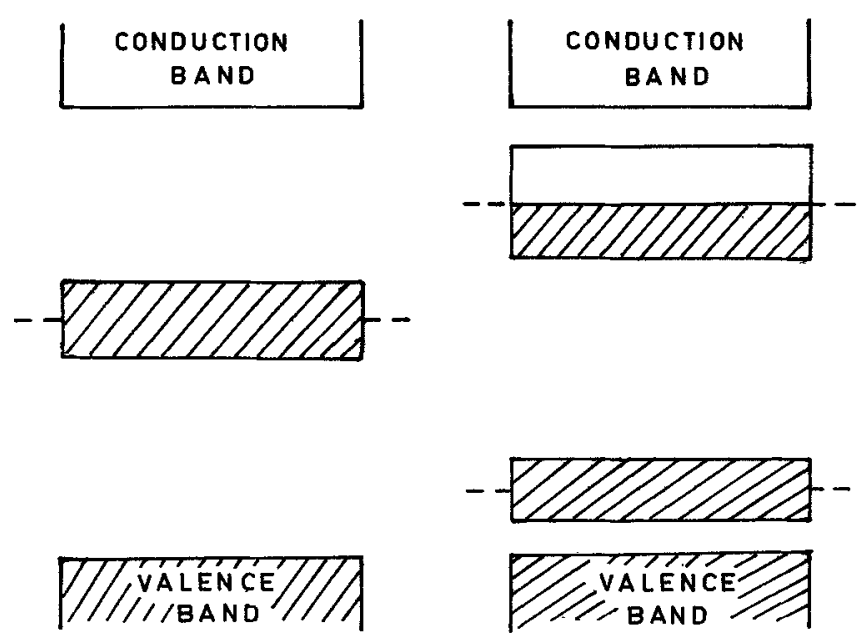

(a)

(b)

Figure 24. Band diagram for first-order transition from an insulating to a metallic state in doped polyacetylene. a. Soliton-lattice model in which the impurity band in the middle of the band gap is full and the polymer is not conducting and b. polaron lattice model in which excessive doping produces a half-full upper polaron band and a full lower polaron band, and the polymer is conducting. The transition is a cross over from a lattice of charged solitons to a regular array of polaron-like distortions, a polaron being the combination of the electron and its strain field. 
the nondestructive testing of polymer-based composite materials (Green 1990). When such materials are damaged by impact the existence and extent of damage cannot often be accessed and assessed by mere examination of the outer surface. However, by doping each layer of the polymer-based composite with a different dopant prior to lay-up, subsequent damage can be quantitatively determined as a function of depth into the composite by means of scanning EPR (figure 26). Thus both the spatial position of the dopant as well as its quantity are located (see § 12).

\section{Glasses and ceramics}

\subsection{Glasses}

Glasses form an expansive family of advanced materials which can be characterized by means of EPR. There are three groups of interest: (i) metallic glasses such as $\mathrm{Fe}_{80} \mathrm{~B}_{20}$ and its ternary variants, which are soft amorphous ferromagnets (Bhagat 1973), (ii) semiconducting glasses such as $\mathrm{V}_{2} \mathrm{O}_{5}-\mathrm{TeO}_{2}$ which are hopping $\left(\mathrm{V}^{4+} \rightarrow \mathrm{V}^{5+}\right)$ electronic conductors or chalcogenide glasses (e.g. Se, $\mathrm{As}_{2} \mathrm{~S}_{3}$ ) (Bishop et al 1977), and (iii) insulating glasses such as (a) soda-lime silicate glasses, containing paramagnetic impurities (e.g. $\mathrm{Fe}^{3+}$ ) in trace quantities (Griscom 1980; Rao and Rao 1985) or else radiation induced paramagnetic centres (Griscom 1973/74), (b) soda-borate glasses of variable composition with a substitutional amount of a third component such as $\mathrm{MoO}_{3}$ (up to $25 \mathrm{~mol} \%$ ), $\mathrm{Mo}^{4+}$ being an excellent sensor for the structure of borate glasses (Simon and Nicula 1983).

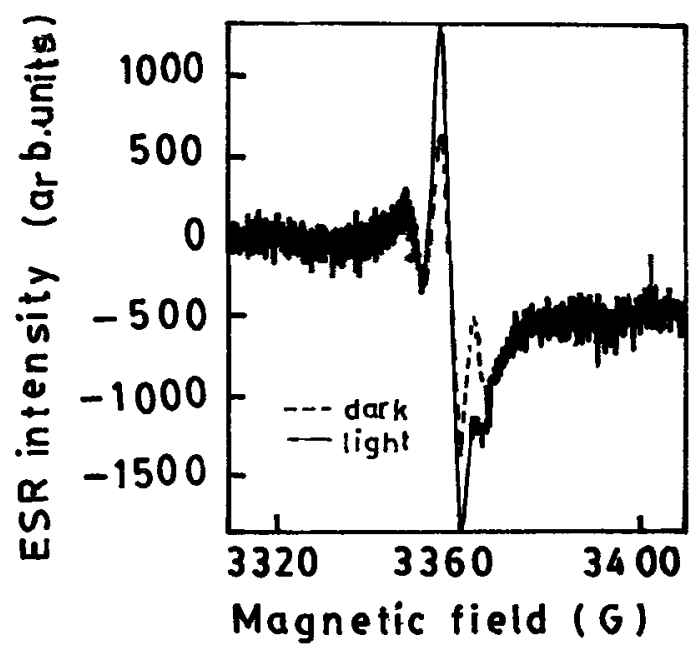

Figure 25. Photo-induced electron transfer from MEH-PPV (donor) to $\mathrm{C}_{60}$ (acceptor) studied through ESR. The two signals: one weaker and wider and the other stronger and sharper induced upon $2.14 \mathrm{eV}$ laser beam irradiation at $80 \mathrm{~K}$ are due to (MEH-PPV) ${ }^{+}$and $\mathrm{C}_{60}^{-}$respectively. The signals disappear at $200 \mathrm{~K}$.

\subsubsection{Metallic glasses}

Metallic glasses are structurally disordered materials obtained by rapid quenching of melts, and are obtainable as wires and ribbons. They are an important class of soft ferromagnetic materials that find application as formers in transformer cores. A ferromagnetic, either crystalline or glassy, unlike a paramagnet, possesses a net nonzero internal magnetic field due to (i) intrinsic spontaneous magnetization, (ii) intrinsic magnetocrystalline anisotropy, (iii) intrinsic surface magnetization, (iv) extrinsic elastic stresses on the sample and (v) skin effect. The presence of magnetostriction tends to make the orientation of the spontaneous magnetization strongly dependent on the magnitude and direction of the internal and external elastic stresses. From the resonance point of view, metallic glasses form a complex system of strongly interacting electrons, complex exchange (electrostatic) interaction which gives rise to the strong internal magnetic field mentioned above. Thus the resonance condition for ferromagnetic samples becomes

$$
\hbar \omega=g \mu_{\mathrm{B}} H_{\mathrm{eff}},
$$

where $H_{\text {eff }}$ is the effective field acting on the precessing magnetic moments and $\omega=2 \pi \nu$. Thus the true resonance field and the intrinsic linewidth - the two important experimental quantities - depend on $H_{\text {eff }}$ to a lesser or greater extent. In fact

$$
H_{\text {eff }}=H_{\text {app }}-H_{\text {dem }}+H_{\text {ani }}
$$

where, $H_{\text {app }}$ is the applied dc magnetic field, $H_{\text {dem }}$ the demagnetizing field, a function of sample dimension

$$
=8 M_{\mathrm{s}} \cot ^{-1}\left[\left(a^{2}+b^{2}+c^{2}\right)^{1 / 2} c / a b\right]
$$

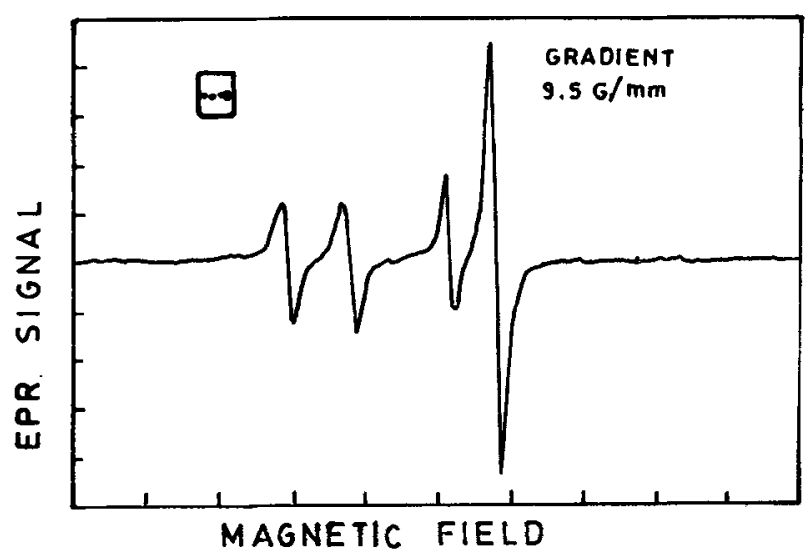

Figure 26. EPR image of organometallic dopant-enhanced damage sites in polymer-based composite. The dopant was applied at four different positions as indicated in the small box. Note that the signal intensity is proportional to the spatial variation of the dopant (Green 1990). 
$a, b, c$ being the width, thickness and length of a rectangular sample and $M_{\mathrm{s}}$ being the saturation magnetization and $H_{\mathrm{an}}$ the anisotropy field.

The so-called Kittel (1947) equation

$$
(\omega / \gamma)^{2}=H_{\mathrm{eff}}\left(H_{\mathrm{eff}}+4 \pi M_{\mathrm{s}}\right)
$$

gives the centre of the ferromagnetic resonance line. Accurate determination of the line centre yields material parameter $H_{\mathrm{an}}, M_{\mathrm{s}}$ and $g$. In most of the situations $H_{\mathrm{an}}$ is small and tends to cancel out $H_{\text {dem }}$ (Spano and Bhagat 1981; Venugopal Rao et al 1992) so that FMR can be used to study the temperature dependence of $M_{\mathrm{s}}$ and $g$.

Another important parameter is the peak-to-peak width of the FMR absorption derivative $\left(\Delta H_{\mathrm{pp}}\right) .\left(\Delta H_{\mathrm{pp}}\right)$ is a function of both temperature and frequency in both crystalline and amorphous ferromagnetic alloys. The temperature dependence may be utilized to characterize the alloy (or in general any magnetic material) as a ferromagnet or as a 'spin glass' (which is a frustrated ferromagnet) as distinct from an antiferromagnet or a paramagnet (figure 27) (Kaul and Siriguri 1992). Note that for a ferromagnet $\Delta H_{\mathrm{pp}}$ rapidly decreases near $T_{\mathrm{c}}$ while for antiferromagnet $\Delta H_{\mathrm{pp}}$ rapidly rises near $T_{\mathrm{N}}$, when $T_{\mathrm{c}}$ and $T_{\mathrm{N}}$ are approached from above. The frequency dependence of the linewidth is a direct consequence of the viscous damping affecting the rotation of the magnetization of the sample around the applied field (Frait and Fraitova 1988). With respect to a coordinate system comprised of $M, M \times H$ and $M x(M \times H)$, the time rate of change of $M$ is given by the Landau-Lifshitz equation

$$
\dot{M}+\gamma\left(M \times H_{\mathrm{eff}}\right)+\frac{\lambda}{\gamma M_{\mathrm{s}}^{2}}(M \cdot \dot{M})+\frac{2 A \gamma}{M_{\mathrm{s}}^{2}}\left(M \times \nabla^{2} M\right),
$$

where $\lambda$ is the Gilbert damping parameter, $A$ the macroscopic exchange stiffness parameter, and $\gamma$ the $g|e| / 2 m c$, the gyromagnetic ratio and the other parameters are defined before.

The last term of (82) is the torque on $M$ due to spatial variation in $M$ and is thus proportional to $A . A$ is important for crystalline ferromagnets which have a two-order of magnitude larger conductivity and thus smaller skin depth at microwave frequencies. Equation (83) when solved using Maxwell's equation yield

$$
\Delta H_{\mathrm{pp}}=\frac{1.45 \lambda \omega}{\gamma^{2} M_{\mathrm{s}}}
$$

An accurate determination of microwave frequency and $H_{\text {eff }}$ and, an assumption of $g$-value enable $4 \pi M_{\mathrm{s}}$ to be determined directly from (82), while (84) yields $\lambda$ from an accurate measurement of $\Delta H_{\mathrm{pp}}$. An alternative, definitive method of determining $M_{s}$ and $\gamma$ (or $g$ ) is to perform FMR experiments at a minimum of three frequencies (Bhagat 1973) and to exploit (82) to make a plot of $\omega^{2} / H_{\text {app }}$ vs $H_{\text {app }}$ and obtain $\gamma$ (or $g$ ) from the slope and $M_{s}$ from the intercept. Table 10 summarizes the FMR data on selected amorphous and crystalline ferromagnets. Thus FMR is a relatively rapid and effective method of characterizing metallic glasses. Of course it has also been used to establish magnetic phase diagrams of these alloys (Bhagat et al 1987) and to evaluate critical point exponents for spontaneous magnetization and zero field magnetic susceptibility (Kaul and Babu 1992).

\subsubsection{Semiconducting glasses}

10.1.2a Oxide glasses: Binary oxide glasses that have a transition metal oxide such as $\mathrm{V}_{2} \mathrm{O}_{5}$ or $\mathrm{WO}_{3}$ as a major constituent and $\mathrm{B}_{2} \mathrm{O}_{3}$ or $\mathrm{TeO}_{2}$ as a minor constituent exhibit semiconducting properties that arise from the existence of more than one valence state $\left(\mathrm{V}^{5+}\right.$ and $\mathrm{V}^{4+}$ in the case of $\mathrm{V}_{2} \mathrm{O}_{5}$ ). The continuous hopping of electrons between these states lead to the formation of small polarons. The electrical conduction in these glasses has been explained (Mott 1968) by assuming a random distribution of transition metal ions in the glass network. Nonlinear switching and memory effects occur in these glasses (Kiss and Szorenyi 1977).

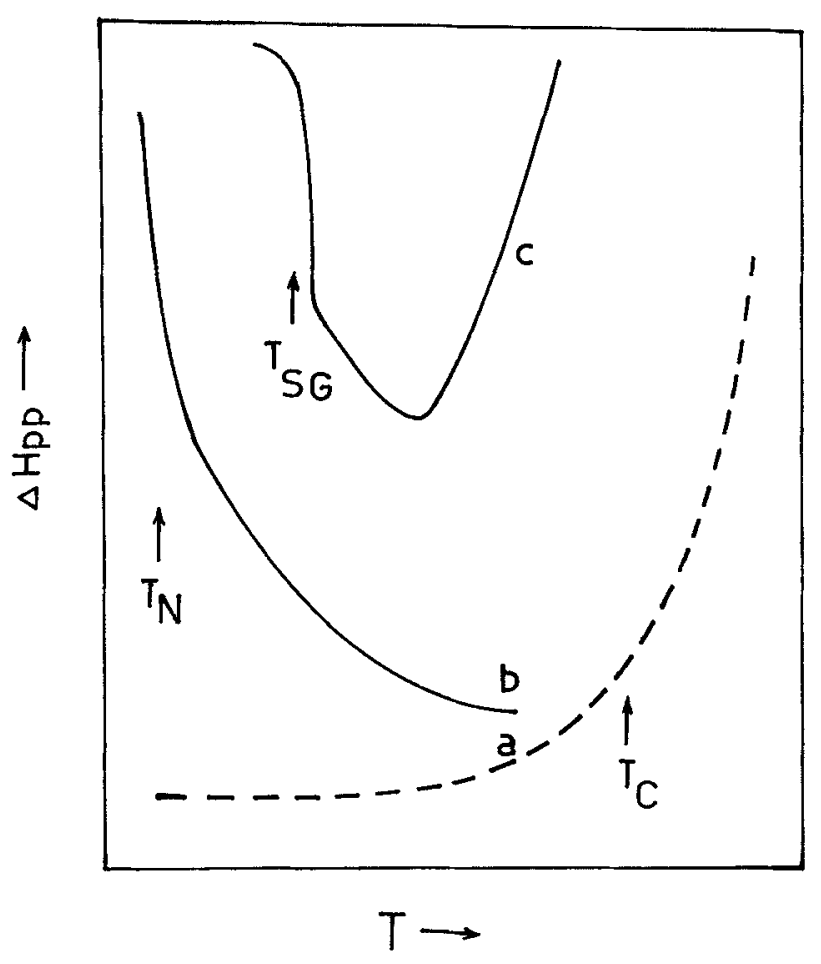

Figure 27. Characterization of magnetic behaviour of amorphous metals through temperature dependence of FMR linewidth $\left(\Delta H_{\mathrm{pp}}\right)$ a. a ferromagnet shows a drastic reduction in linewidth when cooled through $T_{\mathrm{c}}$, while $\mathbf{b}$. an antiferromagnet shows a drastic increase in $\Delta H_{p p}$ upon cooling through $T_{N}$. However, a 'spin glass' initially behaves like a ferromagnet but when cooled through the spin glass temperature, exhibits a trend akin to an antiferromagnet but with a steeper ascent. 
Using the intrinsic $\mathrm{V}^{4+}\left(3 \mathrm{~d}^{l}\right)$ as the paramagnetic probe the ESR spectra of $55 \mathrm{~mol} \% \mathrm{~V}_{2} \mathrm{O}_{5}-45 \mathrm{~mol} \% \mathrm{MO}_{2}$ $(\mathrm{M}=\mathrm{Ge}, \mathrm{Se}, \mathrm{Te})$ have been examined in the temperature range 298-498 K (Sunandana and Bhatnagar 1984). The three spectra at $298 \mathrm{~K}$ (figure 28 ) are characterized by a typical axially symmetric profile due to $3 \mathrm{~d}^{1}(S=1 / 2)$ electron interacting with a ${ }^{51} \mathrm{~V}(I=7 / 2)$ nucleus giving rise to a well-resolved (for $M=G e$ ) to poorly-resolved $(\mathrm{M}=\mathrm{Se})$ pattern of 8 'parallel' and 8 'perpendicular'. The ESR parameters of selected semiconducting glasses are given in table 11. These differences are attributed to a monotonic increase in the covalency of the $\mathrm{V}-\mathrm{O}$ bond on going from $\mathrm{GeO}_{2}$ to $\mathrm{TeO}_{2}$ to $\mathrm{SeO}_{2}$. All the three compositions show dramatic but reversible temperature dependence by way of progressive broadening and eventual disappearance of the vanadium hyperfine structure. This behaviour has been attributed to a thermally-activated delocalization of the $3 \mathrm{~d}^{1}$ electron spin leading to hopping $\mathrm{V}^{4+}$ electron conduction. The broadening of the hyperfine structure implies that at high temperatures the hopping rate of $\mathrm{V}^{4+}$ ions is of the same order as the hyperfine frequency.

A comprehensive ESR investigation of the pseudoternary glass system $(\mathrm{CuO})_{x}\left(\mathrm{~V}_{2} \mathrm{O}_{5}\right)_{0.55-x}\left(\mathrm{TeO}_{2}\right)_{1-45}(x=0$, $0.005,0.01,0.05,0.1,0.2$ and 0.25 ) aided by electrical conductivity measurements (Sunandana and Rao 1985) has revealed that (i) addition of a small amount $(0.5 \mathrm{~mol} \%)$ of $\mathrm{CuO}$ has the effect of oxidizing $\mathrm{V}^{4+}$ to $\mathrm{V}^{5+}$ and thus suppressing the $\mathrm{V}^{4+}$ resonance and electrical conductivity, (ii) large concentrations of $\mathrm{CuO}(>20 \mathrm{~mol} \%$ ) produces exchange effects of the antiferromagnet type, and $g$-shift of $\mathrm{Cu}^{++}$ESR, with the ESR line too broad

Table 10. Characteristic parameters for certain amorphous ferromagnetic materials determined from ferromagnetic resonance.

\begin{tabular}{|c|c|c|c|c|c|c|}
\hline \multirow[b]{2}{*}{ Material } & \multirow{2}{*}{$\begin{array}{r}T_{\mathrm{c}} \\
(\mathrm{K})\end{array}$} & \multirow[b]{2}{*}{$g$} & \multirow{2}{*}{$\underset{(\mathrm{mT})}{M_{\mathrm{s}}}$} & \multicolumn{2}{|c|}{$\lambda\left(10^{8} \mathrm{~s}^{-1}\right)$} & \multirow{2}{*}{$\underset{\left(\mathrm{J} / \mathrm{m}^{3}\right)}{K_{\mathrm{u}^{3}}}$} \\
\hline & & & & $300 \mathrm{~K}$ & $77 \mathrm{~K}$ & \\
\hline $\mathrm{Fe}_{90} \mathrm{Zr}_{10}^{\mathrm{a}}$ & $240 \pm 1$ & $2 \cdot 07 \pm 0 \cdot 02$ & $90 \pm 1$ & $5 \cdot 18$ & - & $29 \pm 4$ \\
\hline $\mathrm{Fe}_{91} \mathrm{Zr}_{9}^{\mathrm{a}}$ & $212 \pm 1$ & $2.07 \pm 0.02$ & $89 \pm 1$ & 5.07 & - & $16 \pm 2$ \\
\hline $\mathrm{Co}_{90} \mathrm{Zr}_{10}$ & 00 & $2.09 \pm 0.02$ & - & - & - & - \\
\hline \multicolumn{7}{|c|}{$\mathrm{Fe}_{75-x} \mathrm{Ni}_{x} \mathrm{~B}_{25}^{\mathrm{b}}$} \\
\hline$x=35$ & $637 \pm 10$ & & $76 \pm 1$ & $1 \cdot 17(8)$ & $1 \cdot 36(9)$ & - \\
\hline$x=45$ & $539 \pm 10$ & & $72 \pm 0.5$ & $0.80(7)$ & $0.97(8)$ & - \\
\hline$x=55$ & $373 \pm 10$ & & $51.5 \pm 0.5$ & $0.42(3)$ & $0.70(6)$ & - \\
\hline \multicolumn{7}{|c|}{$\mathrm{Fe}_{83-y}, \mathrm{Wy} \mathrm{B}_{17}^{\mathrm{b}}$} \\
\hline$y=5$ & $450 \pm 10$ & & $106 \cdot 6 \pm 0 \cdot 4$ & $1 \cdot 28(20)$ & $1.79(25)$ & - \\
\hline$y=9$ & $354 \pm 10$ & & & $1 \cdot 36(14)$ & $2 \cdot 48(26)$ & - \\
\hline \multicolumn{7}{|c|}{$\mathrm{Fe}_{20} \mathrm{Ni}_{60} \mathrm{~B}_{20}^{\mathrm{c}}$} \\
\hline & $93 \mathrm{GHz}$ & $420 \pm 5$ & $2 \cdot 1$ & $500 \cdot 8$ & $1 \cdot 3$ & - \\
\hline \multicolumn{7}{|c|}{$\left(\mathrm{Fe}_{x} \mathrm{Ni}_{1-x}\right)_{75} \mathrm{P}_{16} \mathrm{~B}_{6} \mathrm{Al}_{3}^{\mathrm{d}}$} \\
\hline$x=0.2$ & 92 & - & $26 \cdot 3$ & - & - & - \\
\hline$x=0.25$ & 154 & 2.08 & $38 \cdot 2$ & - & - & - \\
\hline$x=0.30$ & 221 & 2.08 & $43 \cdot 0$ & - & - & - \\
\hline$x=0.35$ & 280 & 2.06 & $47 \cdot 7$ & - & - & - \\
\hline$x=0.50$ & 440 & $2 \cdot 08$ & $66 \cdot 0$ & - & - & - \\
\hline \multicolumn{7}{|c|}{$\left(\mathrm{Co}_{x} \mathrm{Ni}_{1-x}\right)_{75} \mathrm{P}_{16} \mathrm{~B}_{6} \mathrm{Al}_{3}^{\mathrm{d}}$} \\
\hline$x=0.40$ & 88 & - & $15 \cdot 9$ & - & - & - \\
\hline$x=0.50$ & 180 & $2 \cdot 15$ & 27.8 & - & - & - \\
\hline$x=0.60$ & 264 & $2 \cdot 13$ & $35 \cdot 0$ & - & - & - \\
\hline$x=1.00$ & 630 & 2.09 & $70 \cdot 8$ & - & - & - \\
\hline $\mathrm{Fe}^{\mathrm{b}}$ & 1043 & & $175 \cdot 2$ & 0.70 & 0.70 & \\
\hline
\end{tabular}

${ }^{\mathrm{a}} \mathrm{Kaul}$ and Siriguri (1992)

${ }^{b}$ Kaul S N and Mohan Babu T V S M 1989 J. Phys. Condens. Matter 18509

${ }^{c}$ Venugopal Rao et al (1992)

dSpano and Bhagat (1981) 
to be detectable at $289 \mathrm{~K}$ for a CuO content of $25 \mathrm{~mol} \%$. A structural model, showing probable path of hopping is shown in figure 29. While the hopping could be from $\mathrm{V}^{4+}$ to $\mathrm{V}^{5+}$, the interactions between $\mathrm{Cu}^{2+}$ and $\mathrm{V}^{4+}$ would probably be mediated by $\mathrm{Cu}^{2+}---\mathrm{O}^{2-}---\mathrm{V}^{4+}$ superexchange mechanism.

An investigation of pseudobinary glass (0.5 MO) $\left(0.50 \quad \mathrm{~V}_{2} \mathrm{O}_{5}\right) \quad\left(0.45 \quad \mathrm{TeO}_{2}\right),\left(\mathrm{MO}=\mathrm{TiO}_{2}, \mathrm{Cr}_{2} \mathrm{O}_{3}, \mathrm{MnO}_{2}\right.$, $\mathrm{Fe}_{2} \mathrm{O}_{3}$ and $\mathrm{Co}_{2} \mathrm{O}_{3}$ ), with the $3 \mathrm{~d}$-transition metal ion partially substituting $\mathrm{V}$-ion has shown (Sunandana 1987) that while $\mathrm{Ti}^{3+}, \mathrm{Cr}^{3+}$ and $\mathrm{Co}^{3+}$ modify the geometry of the paramagnetic complex and thus control the electron hopping process, introduction of $\mathrm{Mn}^{2+}$ and $\mathrm{Fe}^{3+}$ leads to formation of paramagnetic clusters.

10.1.2b Chalcogenide glasses: ESR signals in pure chalcogenide glasses (Bishop et al 1977) are generally observed only upon optical excitation. The ground electronic states of these materials are invariably diamagnetic due to spin pairing $(S=0)$. Amorphous $\mathrm{Se}$, As and $\mathrm{Ge}$ chalcogenide glasses all exhibit a broad $(\approx 50 \mathrm{mT}$ wide) resonance with $g=2$ due to unpaired spin localized on

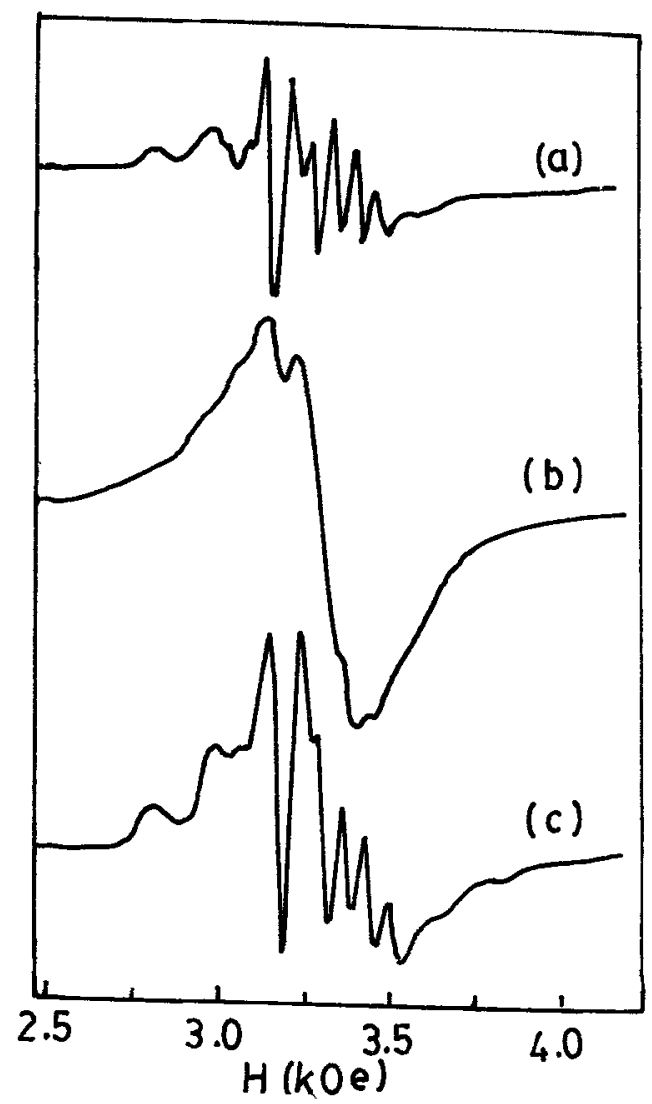

Figure 28. ESR spectra of $55-45$ (a) $\mathrm{V}_{2} \mathrm{O}_{5}-\mathrm{GeO}_{2}$, (b) $\mathrm{V}_{2} \mathrm{O}_{5}-$ $\mathrm{SeO}_{2}$ and (c) $\mathrm{V}_{2} \mathrm{O}_{5}-\mathrm{TeO}_{2}$ glasses at $298 \mathrm{~K}$. The degree of covalency of the $\mathrm{V}-\mathrm{O}$ bands increases in the order $\mathrm{SeO}_{2}<\mathrm{TeO}_{2}<\mathrm{GeO}_{2}$ (Sunandana and Bhatnagar 1984). a single chalcogen atom. Amorphous $A s$ and Ascontaining glasses exhibit an additional, much broader $(\Delta H \sim 150 \mathrm{mT})$ resonance that could arise from an unpaired electron localized on a predominantly s-type orbital of As (reminescent of the $P_{4}^{0}$ centre in amorphous $\mathrm{Si})$, interacting with the ${ }^{75} \mathrm{As}(I=3 / 2)$. This unresolved hyperfine interaction could be resolved through ${ }^{75} \mathrm{As}$ ENDOR studies to obtain the exact neighbourhood of this centre.

Structural imperfections or any deviation from ideal random network structure or from chemically normal bonding expected from chemical composition in these glasses play a significant role in their useful electrical and optical properties. These imperfections usually involve a pair of positively and negatively charged defects. Direct experimental evidence for the presence of these defects help to understand their role in the context of their electrical and optical properties.

Kawazoe et al (1987) investigated, through ESR (i) the type of defects in chalcogenide liquids $\mathrm{S}$ and $\mathrm{As}_{2} \mathrm{~S}_{3}$ and $\mathrm{As}-\mathrm{Ge}-\mathrm{S}$ at a particular temperature, and (ii) the manner in which these defects combine to form normal bonds during the cooling process. In liquid $S$, an almost Lorentzian, singlet homogeneously broadened ESR absorption with $g=2.02$ is observed for temperatures above $160^{\circ} \mathrm{C}$, the temperatures at which $S_{8}$ ring molecules begin to break to form long linear chains. This signal is thus attributed to the formation of dangling bonds in the liquid. The width of the line increases markedly with temperature due to a decrease in the life-time of the radical. Likewise, liquid $\mathrm{As}_{2} \mathrm{~S}_{3}$ gives a broad singlet absorption at temperature greater than $360^{\circ} \mathrm{C}$ with the lineshape changing from Lorentzian to Gaussian around $500^{\circ} \mathrm{C}$. The concentration of these broken bonds upon cooling the melts to form glass is determined by the chemical equilibrium between normal bonding state (As-S), broken bonds (As and S) and homobonds (As-As, S-S). The concentration of these 'chain end radicals' (somewhat like in fractured polymers) increases markedly, reaching $\sim 10 \mathrm{~mol} \%$ at $700^{\circ} \mathrm{C}$.

The enthalpy difference between normal bonds and homobonds is usually small. Upon cooling the melts, while the radical species disappear in the case of $S$ and As-S, a fraction of the neutral defects or $E^{\prime}$ centre $(g$ $\sim 2.01, A \sim 24 \mathrm{mT}$ ) remains in Ge-S, implying that the rate of ${ }^{73} \mathrm{Ge}$ recombination of radical species in $S$ and As is greater than the rate in $\mathrm{Ge}-\mathrm{S}$ by several orders of magnitude. This is attributed to difference in structure and bonding: $S$ and As-S are molecular systems with intra-molecular van der Waals bonds, while $\mathrm{GeS}_{2}$ has a rigid three-dimensional cross-linked structure where melting is possible only by breaking strong $\mathrm{Ge}-\mathrm{S}$ bonds. Thus this study has (i) established that the formation of positively and negatively charged species is less probable than the facile radical in chalcogenide liquids, and (ii) 
highlighted the crucial role of hierarchy of chemical bond strengths in determining the structure of glass forming chalcogenide liquids.

ESR studies on transition-metal-ion-doped Ge chalcogenide glasses have focused on aspects of local structure and bonding. In $\mathrm{Ge}_{x} \mathrm{Se}_{1-x}(0 \leq x \leq 0.42)$, containing 0.01 at.\% Mn (Durny 1980), two characteristic resonances, one at $g=4.3$ with resolved hyperfine structure due to ${ }^{55} \mathrm{Mn} \quad(I=5 / 2)$ and the other at $g=2.0$ with hyperfine structure $(A)$ rarely resolved are found. In this case $A=(51 \pm 5) \times 10^{-4} \mathrm{~cm}^{-1}$, which is correlated to the structure through the Hannay-Smith relation which relates the degree. of covalency of the Mn complex (C) with the electronegativity difference between the $\mathrm{Mn}$ impurity and the chalcogen neighbours (L), $x_{\mathrm{L}}-x_{\mathrm{Mn}}$

$$
C=1-0 \cdot 16\left(x_{\mathrm{L}}-x_{\mathrm{Mn}}\right)-0.35\left(x_{\mathrm{L}}-x_{\mathrm{Mn}}\right)^{2} .
$$

To get the number of nearest neighbours around $(n)$, Pauling covalency $c / n$ is plotted against $A$. The abovementioned $A$ value is compatible with $n=4$ so that $\mathrm{Mn}$ sites occupy $\mathrm{Ge}$ sites in the Ge-Se network with four Se neighbours. $\mathrm{Mn}^{++}$in $\mathrm{Ge}_{1(x)-x} \mathrm{Sn}(x=50,54,58,62$, 66.7) gives rich ESR spectra (Watanabe et al 1978) with the composition $x=58$ and 66.7 giving as many as four times at $g=2 \cdot 0,2 \cdot 8,4 \cdot 3$ and $6 \cdot 0$, suggesting a large variety of internal electric fields of distinct symmetry in these glass compositions, while the $x=50$ composition gives only the $g=2.0$ line, corresponding to $\mathrm{Mn}^{++}$, in nearly cubic symmetry. The two other compositions $x=54$ and 62 give two resonances at $g=4.3$ and $g=2.0$ with the latter exhibiting a hyperfine structure of magnitude $(65 \pm 5) \times 10^{-4} \mathrm{~cm}^{-1}$.

$\mathrm{Ge}_{x} \mathrm{Se}_{1-x}(23 \leq x \leq 44)$ glasses containing 0.5 at.\% $\mathrm{Ti}$ (Zhilinskaya et al 1991) give, when $n$ lies between 32.5 and 41 , a line with $g_{\mathrm{av}}=1.982$, due to $\mathrm{Ti}^{3+}\left(3 \mathrm{~d}^{\prime}\right)$ in a tetragonally-compressed octahedral environment. The concentration of this centre increases upon increasing $x$. For $x \leq 33 \cdot 3$, an intrinsic paramagnetic centre called types $I$ and $I^{\prime}$ with principal $g$ values 2.040, 2.026, 2.0036 and $2.051,2.025,2.0036$, corresponding to cis- and transconfigurations of $S$ in the Ge-S glass network is formed. While doping with $\mathrm{Ti}$ or/and $\mathrm{Cu}$ does not alter these $g$-parameters, $\mathrm{Ti}$ doping decreases, increases or does not affect the intensity depending on glass composition, and $\mathrm{Cu}$-doping decreases the concentration of $\mathrm{Ti}^{3+}$ centres.

\subsubsection{Insulating glasses}

10.1.3a Photochromic glasses: The photochromic glasses have the characteristic property of spatial variation of optical absorption. They are produced by the precipitation of $\mathrm{Cu}(\mathrm{I})$-doped $(0.01 \%)\left(\mathrm{AgCl}_{x} \mathrm{Br}_{1-x}\right)(\sim 1 \%)$ silver halide microcrystals $(\sim 100 \AA$ in size) in an aluminoboro-silicate glass matrix. The photochromic darkening, due to photolysis of silver halide by UV light, a process akin to photographic latent image formation (Sahyun 1992), is enhanced by $\mathrm{Cu}$, a well-known sensitizer. $\mathrm{Cu}$ exists as optically inactive but ESR active $\mathrm{Cu}^{++}$in the glass matrix and as optically active but ESR inactive $\mathrm{Cu}^{+}$in $\mathrm{Ag}$-halide particles. The reactions that describe the darkening mechanism are:

$$
\begin{aligned}
& \mathrm{Ag}^{+}+\mathrm{Cl}^{-} \stackrel{h v}{\longrightarrow} \mathrm{Ag}^{0}+\mathrm{Cl}^{\prime \prime}, \\
& \mathrm{Ag}^{+}+\mathrm{Cu}^{+} \stackrel{\text { hv }}{\longrightarrow} \mathrm{Ag}^{+}+\mathrm{Cu}^{++}
\end{aligned}
$$

The glass matrix does not allow excess $\mathrm{Cl}^{\circ}$ to escape so that the darkening is reversible. While the optical absorption of colloidal silver $(\sim 10-15 \AA$ size $)$ formed by $\mathrm{Ag}^{\mathrm{n}}$ aggregates on silver halide surface constitutes darkening i.e. brown colouration of glass, some of the

\begin{tabular}{|c|c|c|c|c|c|}
\hline Second component & $g_{\|}( \pm 0.005)$ & $g_{1}(0.005)$ & $A_{\|}(\mathrm{mT} \pm 0 \cdot 1)$ & $A_{\perp}(\mathrm{mT} \pm 0.1)$ & $A_{\mathrm{iss}}(\mathrm{mT})$ \\
\hline $\mathrm{GeO}_{2} 950^{\circ} \mathrm{C}$ & 1.908 & 1.984 & 17.9 & $6 \cdot 7$ & 104 \\
\hline $750^{\circ} \mathrm{C}$ & 1.915 & 1.966 & 17.5 & 6.9 & 104 \\
\hline $\mathrm{SeO}_{2}$ & 1.974 & 1.993 & $15 \cdot 6 \pm 1$ & $7 \cdot 5 \pm 1$ & 102 \\
\hline $\mathrm{TeO}_{2}$ & 1.921 & 1.990 & 17.7 & $6 \cdot 5$ & 102 \\
\hline $\mathrm{SrO}$ & 1.993 & 1.982 & 17.6 & $5 \cdot 7$ & 97 \\
\hline $\mathrm{Sb}_{2} \mathrm{O}_{3}$ (cryst.) & 1.933 & 1.984 & $19 \cdot 3$ & 7.5 & 114 \\
\hline 一 & 1.936 & 1.987 & $20 \cdot 1$ & $6 \cdot 7$ & 112 \\
\hline $\mathrm{P}_{2} \mathrm{O}_{5}(8 \%)$ & 1.931 & 1.984 & $20 \cdot 1$ & $7 \cdot 2$ & 115 \\
\hline $\mathrm{P}_{2} \mathrm{O}_{5}(20 \%)$ & 1.950 & 2.000 & $16.8 \pm 0.8$ & $7 \cdot 1 \pm 0.5$ & 103 \\
\hline $\mathrm{P}_{2} \mathrm{O}_{5}(50 \%)^{+}$ & 1.938 & 1.970 & $19 \cdot 8$ & $6 \cdot 0$ & 106 \\
\hline $\mathrm{B}_{2} \mathrm{O}_{3}^{*}$ & 1.9212 & 1.9848 & $16 \cdot 26$ & 6.73 & 99 \\
\hline $\mathrm{GeO}_{2}^{*}$ & 1.929 & 1.976 & $15 \cdot 8$ & $6 \cdot 3$ & 95 \\
\hline
\end{tabular}
photoproducts are expected to exhibit ESR.

Table 11. ESR parameters for $\mathrm{V}_{2} \mathrm{O}_{5}-\mathrm{MO}_{2}$ glasses

"Sunandana and Bhatnagar (1984); ${ }^{+}$Hydrated glass; ${ }^{*} \mathrm{~V}^{4+}$ as an impurity. 
Marquardt (1976) detected the ESR of photo-induced $\mathrm{Cu}^{++}$ions distributed among 16 distorted randomlyoriented, approximately axial cation sites in the silver halide phase. These $\mathrm{Cu}^{++}$centres, resembling those in strained $\mathrm{AgCl}$ crystals doped with $\mathrm{Cu}^{++}$, are characterized by $g_{1}=2.05(2), g_{2}=2 \cdot 11(5)$ and $g_{3}=2 \cdot 22(5), A_{1} \leq 2 \mathrm{mT}$, $A_{2} \leq 7.8(0.5) \mathrm{mT}$ and $A_{3}=9(0.5) \mathrm{mT}$ obtained by computer simulation and are to be distinguished from matrix-glassbased $\mathrm{Cu}^{2+}$. A dual-cavity method together with microwave saturation of $\mathrm{Cu}^{++}$was employed to enhance the photo ESR signal.

Recent ESR studies (Caurant et al 1992) on photochromic glasses containing $0.175 \mathrm{wt} . \% \mathrm{Ag}, 0.204 \mathrm{wt} . \%$ $\mathrm{Cl}, 0.135 \mathrm{wt} . \% \mathrm{Br}$ and $0.007 \mathrm{wt} . \% \mathrm{Cu}$ have used a simple method which selectively eliminates the signal of matrix $\mathrm{Cu}^{2+}$ (figure 30). Keeping the dc magnetic field fixed the intensity of $\mathrm{Cu}^{++}$resonance is monitored as a function of irradiation time (mercury lamp light focused through optical fibre). The role of the hole trapped by copper is demonstrated to be both to stabilize such holes and to generate the interstitial silver ions which trap the electrons.

EPR studies of time evolution of photo-induced $\mathrm{Cu}^{++}$ in thermally-bleached silver halide photochrome glasses (Caurant et al 1993) have shown that during exposure to UV light, only one stable $\mathrm{Cu}^{++}$species - a $\mathrm{Cu}^{++}-\mathrm{Ag}$ vacancy complex with silver in a nearest neighbour position $\left(\mathrm{Cu}^{++}-\mathrm{V}_{\mathrm{Ag}}\right)_{A}-$ is formed. It is characterized by $g_{\mathrm{x}}=2.116, g_{\mathrm{y}}=2.244, g_{\mathrm{z}}=2.076$ and $A_{\mathrm{x}}=6.8, A_{\mathrm{y}}=7.0$, $A_{\mathrm{z}}=2.3 \mathrm{mT}$. This centre decays rapidly in the dark, through two parallel mechanisms:

(i) Dislocation of the complex $\left(\mathrm{Cu}^{++}+e^{-} \rightarrow \mathrm{Cu}^{+}\right)$by

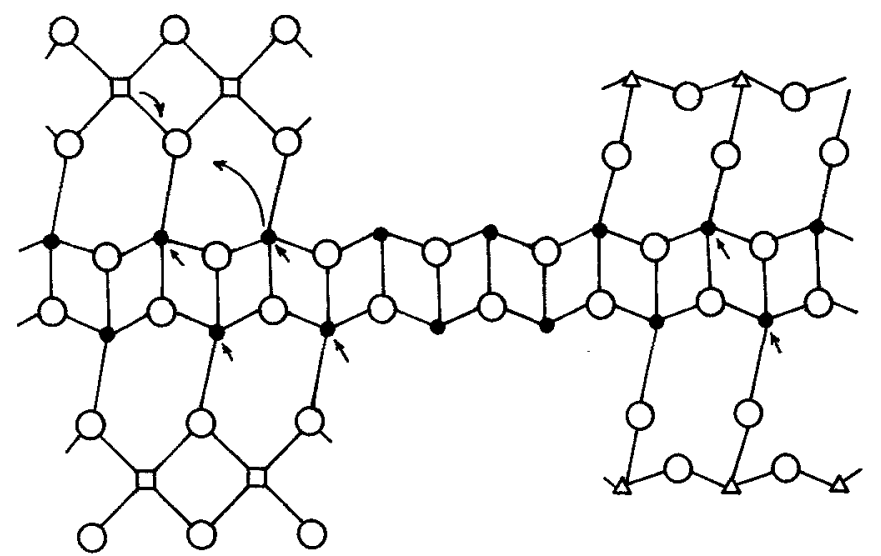

$$
\text { - V } \triangle \text { Te } \square \mathrm{Cu} O \text { o }
$$

Figure 29. A schematic cross-section of the $\mathrm{CuO}-\mathrm{V}_{2} \mathrm{O}_{5}-\mathrm{TeO}_{2}$ glass network showing the direction of the electron hopping. (A direct evidence was obtained from temperature dependent ESR spectra of $\mathrm{V}^{4+}\left(3 \mathrm{~d}^{\prime}\right)$ in $\mathrm{V}_{2} \mathrm{O}_{5}-\mathrm{TeO}$ (Sunandana and Rao 1985)). displacement of vacancy along a (Chen et al 1985) direction in the fcc lattice of $\mathrm{AgCl}_{x} \mathrm{Br}_{1-x}$, with an activation energy of $0.44 \mathrm{eV}$ and a frequency factor $3.4 \times 10^{5} \mathrm{~s}^{-1}$.

(ii) Conversion of $\left(\mathrm{Cu}^{++} \mathrm{V}_{\mathrm{Ag}}\right)_{A}$ to $\left(\mathrm{Cu}^{++} \mathrm{Cl}^{-} \mathrm{V}_{\mathrm{Ag}}\right)_{B}$ where the silver vacancy is in the next nearest neighbour position along (Chen et al 1985) direction, with the same activation energy as $A$ but with a slightly lower frequency factor of $3.1 \times 10^{5} \mathrm{~s}^{-1}$. The $\left(\mathrm{Cu}^{++} \mathrm{Cl}^{-} \mathrm{V}_{\mathrm{Ag}}\right)_{B}$ centre with $g_{\mathrm{x}}=2.066, g_{\mathrm{y}}=2.077, g_{\mathrm{z}}=6.5 \mathrm{mT}$, decays slowly by a vacancy hopping mechanism $\left(\mathrm{Cu}^{++}\right.$ $(B) \rightarrow \mathrm{Cu}^{++}$) with an activation energy $0.22 \mathrm{eV}$ and a frequency factor of $4.6 \mathrm{~s}^{-1}$.

Annihilation of $A$ and $B$ centres via the formation of a $\mathrm{Cu}^{+}$ion and a neutral complex $\left(\mathrm{Ag}^{++} \mathrm{V}_{\mathrm{Ag}}\right)_{A}$ (which migrates to the surface of the silver halide particle,

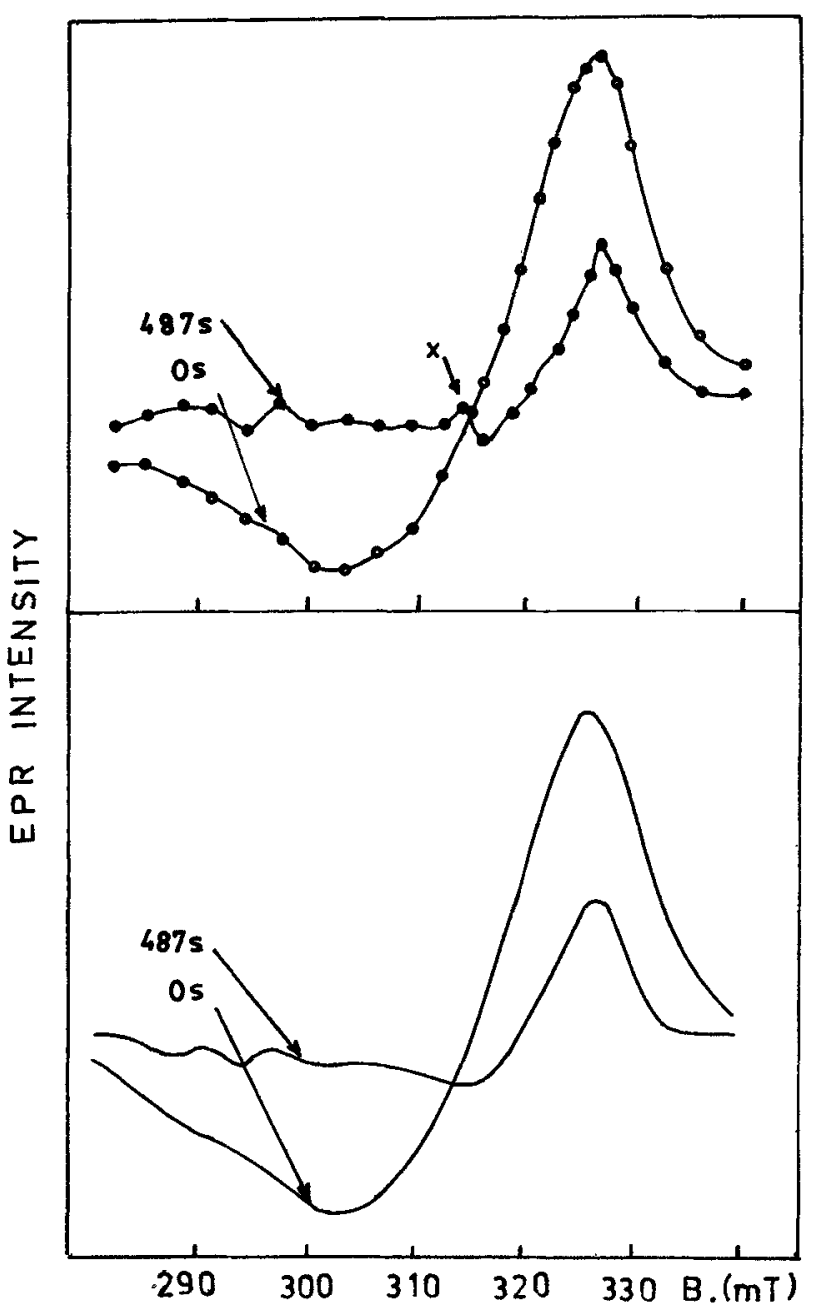

Figure 30. EPR spectra of photo-induced $\mathrm{Cu}^{++}$centres in silver photochromic glass. Top: Experimental spectrum obtained by plotting the intensities as a function of applied field, keeping the bleaching times $487 \mathrm{~s}$ and $0 \mathrm{~s}$. Bottom: Spectra simulated by the sum of two copper centres. ' $X$ ' is an unidentified feature not simulated that appears at the end of bleaching (Caurant et al 1992). 
where electron-hole recombination occurs) explain these two decay channels. This study convincingly demonstrates that the $\mathrm{Cu}^{++}(A)$ centre is the one detected at equilibrium darkening while $\mathrm{Cu}^{++}(B)$ is the one found at the end of thermal bleaching.

10.1.3b Fluoride glasses: Halide glasses in general and fluoride glasses in particular, are an important class of optical materials. Water-free $\mathrm{BeF}_{2}$ glass for instance, is a laser host and a UV window material besides being a core material for ultra-low loss optical fibres. Structurally, $\mathrm{BeF}_{2}$ glass is akin to fused silica so that a study of impurity ESR spectra would offer important clues to local structure. An ESR study of 'distilled' $\mathrm{BeF}_{2}$ glasses containing traces of $\mathrm{Mn}^{++}(13 \mathrm{ppm}), \mathrm{Ni}(<1.9 \mathrm{ppm})$ and Fe (20 ppm) have revealed (Griscom et al 1986) two resonances: (i) $\mathrm{Mn}^{++}$resonance at $g=2.0$ of six primary hyperfine lines with each line showing a clear ${ }^{19} \mathrm{~F}(I=1 / 2)$. Super hyperfine splitting of at least six components due to $F$-neighbours, when studied at $35 \mathrm{GHz}$ (figure 31) and (ii) an FMR signal due to precipitated heterogeneous magnetite-like phases similar to $\mathrm{NiFe}_{2} \mathrm{O}_{4}$.

A computer simulation of the $\mathrm{Mn}^{++}$spectrum based on an isotropic ${ }^{19} \mathrm{~F}$ splitting of $15 \mathrm{G}$ and assuming an octahedral surroundings for $\mathrm{Mn}^{++}$reproduces fairly well the experimental spectrum, leading to the conclusion that the predominant coordination state on $\mathrm{Mn}^{++}$in $\mathrm{BeF}_{2}$ glass is six-fold.

\subsection{Ceramics}

Ceramics are ubiquitous materials with uses ranging from capacitors and support for catalysts to devices based on high temperature superconductors. Paramagnetic centres occur in these materials as trace impurities or as deliberately doped additives or else as defects created as a

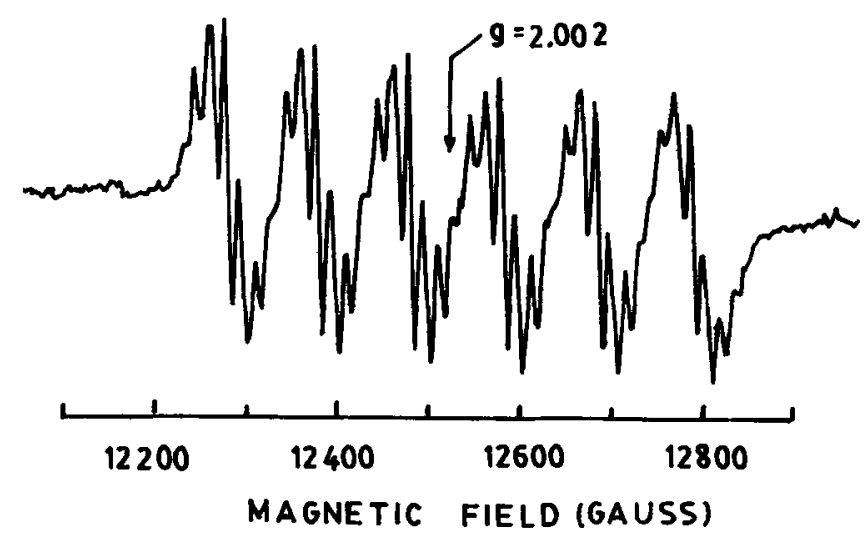

Figure 31. A $35 \mathrm{GHz}$ ESR spectrum of $\mathrm{Mn}^{++}$in distilled $\mathrm{BeF}_{2}$ glasses, showing the six ${ }^{55} \mathrm{Mn}(I=5 / 2)$ primary hyperfine lines and the superhyperfine splitting of at least six components due to $F$ neighbours in the glass network. result of the processing employed to synthesize or modify them. EPR could be used to monitor either the process itself or to characterize the processed materials.

\subsubsection{Catalytic ceramics}

10.2.1a Shock-modified rutile: High pressure (4.5$27 \mathrm{GPa}$ ) shock-modified rutile is found to exhibit enhanced catalytic activity (Graham et al 1986). Shock compression does not introduce any structural changes but creates anisotropic residual strain leading to the formation of paramagnetic defects. These defects are stable even at $450^{\circ} \mathrm{C}$ where catalytic activity is monitored. ESR studies at liquid helium temperatures reveal unique features of these defects and their relation to enhanced catalytic activity. While the starting powder is white and exhibits no ESR, it turns dark grey upon shock compression, the darkness being a function of shock conditions. When the dark grey powder is annealed in air around $500-600^{\circ} \mathrm{C}$, the original whiteness is restored.

Two intense resonances are produced due to shock compression: an isotropic one with $g=2.0029(3)$ and an axially symmetric defect with $g_{\|}=1.969(5)$, $g=1.937(3)$ for $20 \mathrm{GPa}$ shock. The first one, due to free electrons trapped at a vacancy is less intense (concentration: $3 \times 10^{16}$ ) than the second one, due to a reduction in valence of interstitial $\mathrm{Ti}$ from +4 to +3 . Higher shock pressures do not alter the concentration of the axial defects but introduce a strong dispersion in the lineshape.

10.2.1b Vanadia catalysts: The study of the mechanism of heterogeneous catalysis requires in-depth studies of the active intermediates formed by transition metal ions interacting with various molecules on the surface of a supported oxide catalyst. ESR offers a powerful and sensitive technique with which to determine (i) oxidation states, (ii) surface and bulk coordination, and (iii) the physical form of a transition metal oxide on a diamagnetic support (Kazanskii 1976). This technique has been applied to $\mathrm{V}_{2} \mathrm{O}_{5}$ catalysts supported on $\mathrm{SiO}_{2}, \mathrm{Al}_{2} \mathrm{O}_{3}, \mathrm{MgO}$ as well as $\mathrm{MoO}_{3}$ and $\mathrm{TiO}_{2}$ to obtain specific information about (i) the local structure around the paramagnetic $\mathrm{V}^{4+}$ $\left(3 \mathrm{~d}^{l}\right)$, (ii) the differences in the microstructure arising from the distinct nature of the support and (iii) the so-called 'carrier effect' involving the support and active sites. Results of such investigations allow definite conclusions to be drawn about the reactivity of $\mathrm{V}^{4+}$ with gaseous oxygen formed on the supported catalysts, which is the theme of heterogeneous catalysis.

Vanadia catalysis supported on alumina has been used in a number of oxidation and ammoxidation reactions involving aromatic hydrocarbons and also for reduction of NO with $\mathrm{NH}_{3}$. ESR characterization of the catalyst with respect to the amount of transition metal oxide and 
conditions of reduction is an important first step towards optimization, for end use in an actual reaction.

In a typical study (Chary et al 1984), 3.41-20.11 wt\% $\mathrm{V}_{2} \mathrm{O}_{5}$ prepared by decomposition of $\mathrm{NH}_{4} \mathrm{VO}_{3}$, impregnated on 60-70 mesh $\gamma-\mathrm{Al}_{2} \mathrm{O}_{3}$ support with a pore volume $0.4 \mathrm{~cm}^{3} / \mathrm{g}$ and surface area $188 \mathrm{~m}^{2} / \mathrm{g}$ and reduced by flowing hydrogen $\left(500^{\circ} \mathrm{C}\right)$, was investigated by ESR. While the colour of the catalysts ranged from light green to dark yellow as $\mathrm{V}_{2} \mathrm{O}_{5}$ content was increased, none of the catalysts exhibited any ESR at $300 \mathrm{~K}$, unlike the unsupported $\mathrm{V}_{2} \mathrm{O}_{5}$ which shows a symmetric singlet ESR at $300 \mathrm{~K}$ with $g=2 \cdot 000, \Delta H_{\mathrm{pp}}=17 \mathrm{mT}$ which reduces to $14.2 \mathrm{mT}$ at $109 \mathrm{~K}$. The ESR spectra of the catalysts obtained at $109 \mathrm{~K}$ (figure 32a) are characteristic of isolated $\mathrm{V}^{4+}$ or $(\mathrm{V} \ldots \mathrm{O})^{2+}$ species in a ligand field of axial symmetry produced by a distorted octahedron of oxygen ions (table 12). For $\mathrm{V}_{2} \mathrm{O}_{5}$ content equal to or in excess of $9.84 \%$, the low-field parallel ${ }^{51} \mathrm{~V}(I=7 / 2)$ hyperfine components (figure 32b) of the axial ESR spectra show doublet structure, characteristic of two chemically distinct
$\mathrm{V}^{4+}$ species. The concentration of the second component attains a maximum when the $\mathrm{V}_{2} \mathrm{O}_{5}$ content in the catalyst is $20.11 \%$. The second, weaker set of parallel components, also due to $\mathrm{V}^{4+}$ could arise from a relatively unstable intermediate whose ligands consist of adsorbed oxygen atoms. An examination of the crystal structures of the orthorhombic $\mathrm{V}_{2} \mathrm{O}_{5}$ (figure 32c) and cubic $\gamma-\mathrm{Al}_{2} \mathrm{O}_{3}$ reveals that the two distinct set of hyperfine peaks arise from $\mathrm{V}^{4+}$ occupying octahedral and tetrahedral vacant sites surrounded by oxygens in the $\gamma-\mathrm{Al}_{2} \mathrm{O}_{3}$ lattice. Thus on the surface of $\gamma-\mathrm{Al}_{2} \mathrm{O}_{3}$ one could have $\mathrm{V}^{4+}$ dispersed uniformly as active sites with tetrahedral or square pyramidal surroundings, with dynamic equilibrium between the two and with the number of sites available for occupation depending on the concentration of $\mathrm{V}_{2} \mathrm{O}_{5}$ in the catalyst.

10.2.1c Molybdenum sulphide catalysts: Heterocracking - an important catalytic process in the conversion of crude petroleum to gasoline - uses molybdenum
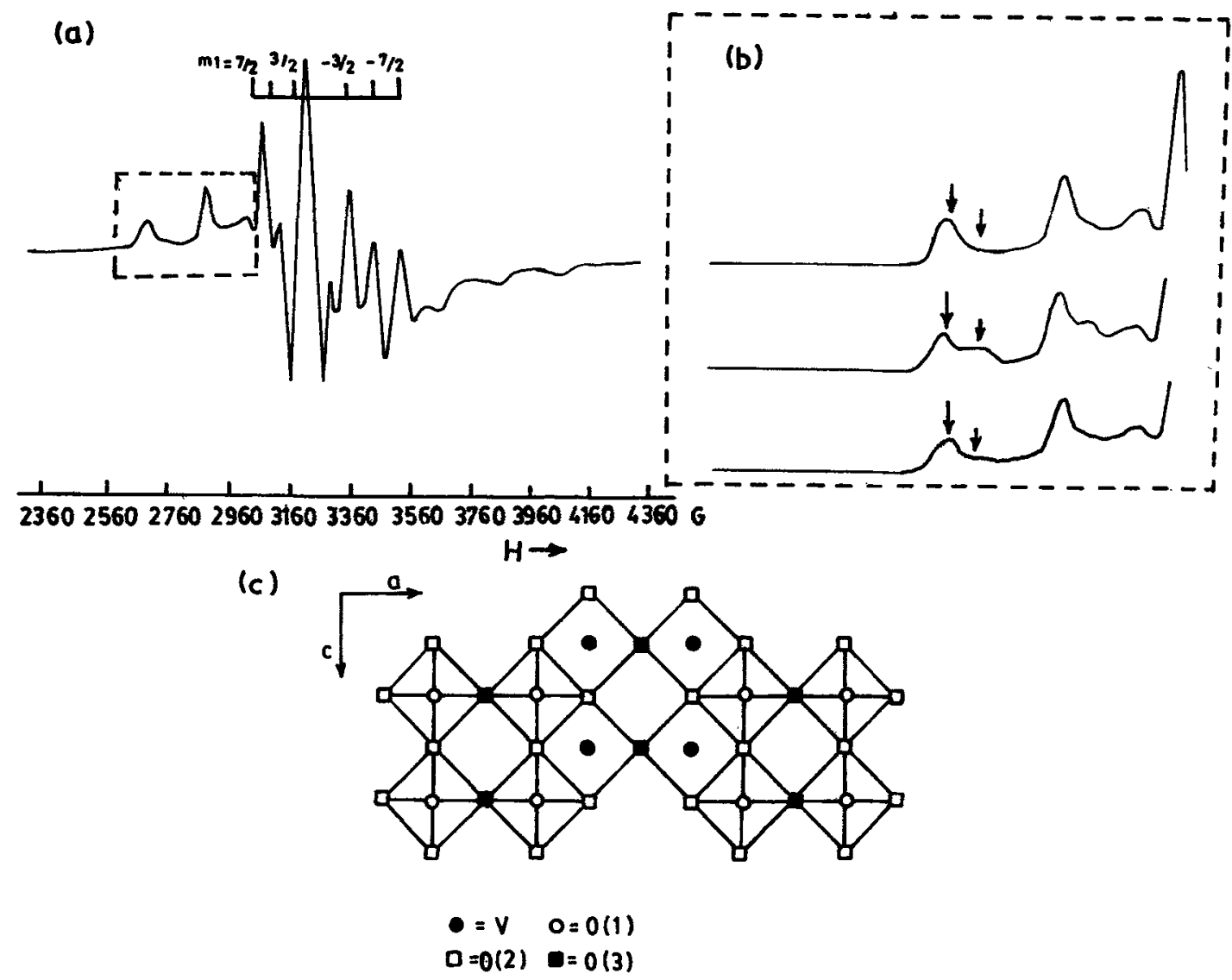

Figure 32. a. ESR spectra of a reduced $3.41 \%$ vanadia catalyst supported on $\gamma$-alumina, at $109 \mathrm{~K}$. The parallel and perpendicular hyperfine structures of ${ }^{51} \mathrm{~V}(I=7 / 2)$ are clearly resolved, b. evolution of the low field parallel ESR peaks (marked area in (a)) as a function of $\mathrm{V}_{2} \mathrm{O}_{5}$ content. Top: $6.96 \%$, middle: $9.84 \%$, bottom: $11.89 \%$. The lengths of arrows indicate relative peak heights of the main and subsidiary $\mathrm{V}^{4+}$ centres and c. schematic structure of $\mathrm{V}_{2} \mathrm{O}_{5}$ in the crystallographic ac plane with $\mathrm{VO}_{5}$ polyhedra idealized to tetragonal pyramids. The $\mathrm{O}(1)$ oxygens forming the strongest bonds with $\mathrm{V}$ are shown as open circles (Chary et al 1984). 
sulphide as a catalyst. The catalyst is prepared from Mo-oxides sulphurized either before melting with petroleum or directly in situ. Usually sulphurization is never complete, and thus the catalytically active compounds are Mo sulphides in which oxygen remains. The electron configuration of the catalyst determines the catalytic process.

ESR measurements of amorphous $\operatorname{MoS}_{2+x}(0 \leq x \leq 1)$ and their compounds $\mathrm{MoS}_{y} \mathrm{O}_{z}$ obtained by oxygen exposure at $130^{\circ} \mathrm{C}$ have revealed the nature and concentration of active sites (Bensimon et al 1992). The ESR spectrum is always the sum of three elementary signals arising from (i) unsaturated sulphur atoms and characterized by $g=2.027 \pm 0.001,2.038$ and 2.034 for $\mathrm{MoS}_{2}$, $\mathrm{MoS}_{2.6}$ and $\mathrm{MoS}_{3}$, respectively, and (ii) $\mathrm{Mo}^{5+}\left(4 \mathrm{~d}^{1}\right.$ ) found in two different surroundings, as reflected by the g-tensors: $A: 0.02,1.99$ and 1.97 and $B: 1.94,1.92$ and 1.89 . As the evolution from $\mathrm{MoS}_{3}$ to $\mathrm{MoS}_{2}$ proceeds, the spectra show a drastic modification around the composition $\operatorname{MoS}_{2.6}$ by way of a 'pseudo-transition' in which a change in the short-range structural order of the amorphous solid occurs. This is confirmed by the influence of oxygen exposure which leads to an increasing number of paramagnetic defects in accordance with catalytic properties.

\subsubsection{Cuprate ceramics}

$\mathrm{CuO}$ and its binary and ternary compounds with $\mathrm{MO}$ $(\mathrm{M}=\mathrm{Mg}, \mathrm{Sr}, \mathrm{Ca}, \mathrm{Ba})$ and $\mathrm{R}_{2} \mathrm{O}_{3}(\mathrm{R}=\mathrm{Y}, \mathrm{La}, \mathrm{Gd}, \mathrm{Nd}$, $\mathrm{Bi}, \mathrm{Tl}$ ) form an extensive class of magnetic (e.g. $\mathrm{Bi}_{2} \mathrm{CuO}_{4}$ antiferromagnetic, $T_{\mathrm{N}}=42 \mathrm{~K}$ ) and superconducting ceramics (e.g. $\mathrm{YBa}_{2} \mathrm{Cu}_{3} \mathrm{O}_{7}$ with $T_{\mathrm{c}} \sim 90 \mathrm{~K}, \mathrm{Bi}_{2} \mathrm{Sr}_{2} \mathrm{CaCu}_{2} \mathrm{O}_{8}$ with $T_{\mathrm{c}}=120 \mathrm{~K}$ ) whose study is crucial to a basic understanding of magnetism and superconductivity, and, eventual applications to high-field magnets and magnetic levitation. Zero-field and low-field microwave absorption and ESR have played a significant role in the characterization of superconducting ceramics.

10.2.2a Cupric oxide: Cupric oxide, the precursor to high- $T_{\text {. }}$ superconductors of the La-, Y-, Bi-, Tl-, and $\mathrm{Hg}$-families, is a monoclinic-structured anti-ferromagnetic insulator, with a Neel temperature $T_{\mathrm{N}}$ of $230 \mathrm{~K}$. A recent ESR study at $45 \mathrm{GHz}$ (Kindo et al 1990), using pulsed magnetic field and a reflection cavity has detected a $1.6 \mathrm{~T}$ wide resonance line at room temperature, with a slight temperature dependence of $g \approx 2.0$ and a lineshape between Lorentzian and Gaussian, and arises from anisotropic exchange interaction between $\mathrm{Cu}^{++}$spins. The linewidth decreases as the temperature is decreased and the line broadens near $T_{\mathrm{N}}$ due to critical slowing down. One-dimensional spin correlation is seen in the ESR line-profile.

The antiferromagnetic coupling between $\mathrm{Cu}$ spins via oxygen atoms is believed to be important for the mechanism of superconductivity in cuprate superconductors.

10.2.2b Ternary cuprates: Compared to CuO, ternary cuprates $\mathrm{SrCuO}_{2}, \mathrm{Sr}_{2} \mathrm{CuO}_{3}, \mathrm{Ca}_{2} \mathrm{CuO}_{3}, \mathrm{Li}_{2} \mathrm{CuO}_{2}$, and $\mathrm{Bi}_{2} \mathrm{CuO}_{4}$ have very small net magnetic susceptibilities and a few of these have very low $T_{\mathrm{N}}$ values. $\mathrm{Li}_{2} \mathrm{CuO}_{2}$, with $T_{\mathrm{N}}=9.3 \mathrm{~K}$ and $\mathrm{Bi}_{2} \mathrm{CuO}_{4}$, are both three-dimensional antiferromagnets. $\mathrm{Bi}_{2} \mathrm{CuO}_{4}$, which has a simple antiferromagnetic order consisting of magnetic moments

Table 12. Spin Hamiltonian parameters of. $\mathrm{V}^{4+}$ in $\mathrm{V}_{2} \mathrm{O}_{5} / \gamma-\mathrm{Al}_{2} \mathrm{O}_{3}^{\#}$.

\begin{tabular}{|c|c|c|c|c|c|}
\hline $\begin{array}{l}\mathrm{V}_{2} \mathrm{O}_{5} \\
\text { content } \\
\text { (wt \%) }\end{array}$ & $\begin{array}{l}\text { Colour of } \\
\text { catalyst }\end{array}$ & $g_{\|}$ & $g_{\perp}$ & $\begin{array}{c}A_{\|} \\
(\mathrm{mT})\end{array}$ & $\underset{(\mathrm{mT})}{A_{\perp}}$ \\
\hline 3.41 & light green & 1.940 & $2 \cdot 000$ & 19.4 & $7 \cdot 1$ \\
\hline 6.96 & light green & 1.939 & 1.997 & 19.4 & $7 \cdot 3$ \\
\hline 9.84 & dark green & $\begin{array}{ll}M & 1.942 \\
S & 1.983\end{array}$ & 2.000 & $\begin{array}{l}19.4 \\
17.5\end{array}$ & $7 \cdot 0$ \\
\hline 11.89 & light yellow & $\begin{array}{lr}\text { M } & 1.942 \\
\text { S } & 1.983\end{array}$ & 1.999 & $\begin{array}{l}19.2 \\
17.0\end{array}$ & $7 \cdot 0$ \\
\hline 13.64 & dark yellow & $\begin{array}{ll}\text { M } & 1.938 \\
\text { S } & 1.971\end{array}$ & 1.995 & $\begin{array}{l}19.4 \\
17.4\end{array}$ & $7 \cdot 1$ \\
\hline $14 \cdot 76$ & dark yellow & M 1.939 & 1.998 & $19 \cdot 2$ & $7 \cdot 2$ \\
\hline $16 \cdot 37$ & dark yellow & $\begin{array}{lr}\text { M } & 1.941 \\
\text { S } & 1.977\end{array}$ & 1.999 & $\begin{array}{l}19.2 \\
17.5\end{array}$ & $7 \cdot 1$ \\
\hline 18.46 & dark yellow & $\begin{array}{ll}M & 1.939 \\
\text { S } & 1.966\end{array}$ & 1.999 & $\begin{array}{l}19.3 \\
18.0\end{array}$ & $7 \cdot 1$ \\
\hline $20 \cdot 11$ & dark yellow & $\begin{array}{ll}\text { M } & 1.939 \\
\text { S } & 1.960\end{array}$ & 1.997 & $\begin{array}{l}19 \cdot 2 \\
18.0\end{array}$ & $7 \cdot 1$ \\
\hline
\end{tabular}

"Chary et al (1984); M: Main centre; S: additional centre. 
aligned ferromagnetically along c-axis and antiferromagnetically between spins at the corner and body-centre sites of the orthorhombic crystal lattice. ESR of the powder at $T>T_{\mathrm{N}}$ at $\mathrm{X}$-band gives a resonance at $g=2.09$, while for a single crystal at $370.4 \mathrm{~K}$ and $265 \mathrm{~K}$ (Ohta et al 1992), an axial spectrum is obtained with $g_{\|}=2.26 \pm 0.01$ and $g_{\perp}=2.04 \pm 0.01$ for $H_{11}$ c-axis and $H_{\perp}$ c-axis, suggesting $\mathrm{Cu}^{++}$situated at an pseudo octahedral site, even though there are only four oxygen ligands for $\mathrm{Cu}^{++}$. The line is Lorentzian and exchange-narrowed and the linewidth $(0.37 \pm 0.03) T$ is almost field independent. An antiferromagnetic resonance mode of the planar type antiferromagnet has been observed below $T_{\mathrm{N}}$.

10.2.2c Cuprate superconductors: Nonresonant microwave absorption in small magnetic fields is one of the most sensitive techniques for observing small amounts
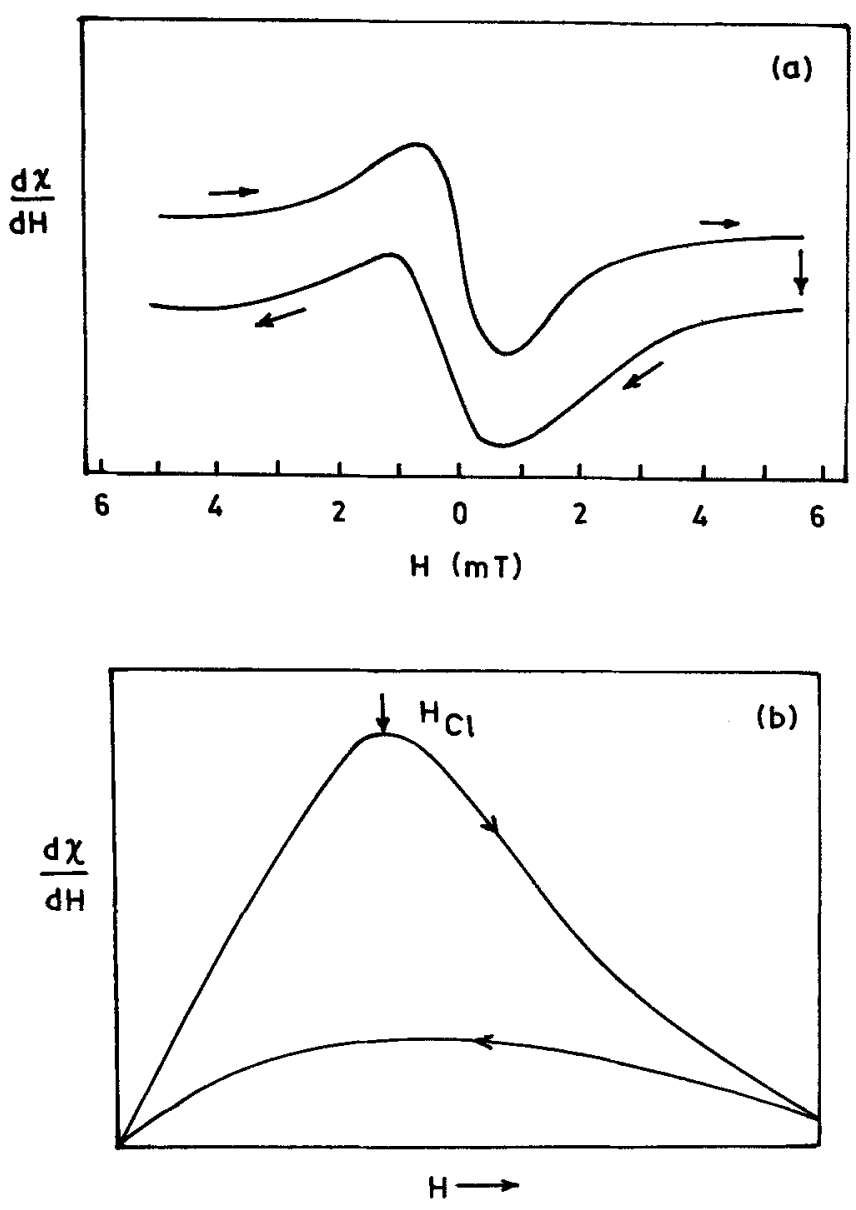

Figure 33. Zero-lield and low-field microwave absorption in high temperature cuprate ceramic superconductors (schematic). a. The frequency-independent, reversible, absorption as the $\mathrm{dc}$ magnetic field is scanned from negative through zero to positive and $\mathbf{b}$. the low-field microwave absorption showing hysteresis. The turning point obtained upon increasing magnetic field from zero corresponds to the lower critical field $\left(H_{c 1}\right)$ which characterizes these granular, type II superconductors. of superconducting phase in an otherwise nonsuperconducting environment (Muller et al 1980; Bhat et al 1987) or small amount of nonsuperconducting impurity in an otherwise superconducting sample, by way of $\mathrm{Cu}^{++}$ESR with $g_{\|}=2.117, g_{\perp}=2.120$ for $\mathrm{BaCuO}_{2}$ (Jones et al 1990). In this simple technique the sample is placed in a microwave cavity at $\sim 9 \mathrm{GHz}$ which is connected to a standard ESR spectrometer. A small magnetic field $( \pm 10 \mathrm{mT})$ and a modulation field parallel to the external field are applied to the sample. The change in the microwave absorption is detected via lock-in amplifier when varying the external field.

The shape of the signal (figure 33a) is characteristic of a granular superconductor in the superconducting state. This frequency independent signal which vanishes above $T_{\text {c }}$ is caused by weak links or Josephson junctions connecting large superconducting areas, and represents the change of the superconducting surface impedance as a function of the applied magnetic field. This technique has been applied (Mehring et al 1993) to the study of the topology of superconducting phase changes in $\mathrm{La}_{2} \mathrm{CuO}_{4}: \mathrm{Sr}$ processing parameters especially the rate of cooling, which decides the size and shape of superconducting region. The low-field microwave absorption (figure 33b), shows a maximum at a field which is recognized as the lower critical field, $H_{c}$, of the superconductor. For $H>H_{\mathrm{c} 1}$ the superconductor enters the 'mixed state' which persists until the upper critical field, $H_{\mathrm{c} 2},\left(H_{\mathrm{c} 2} \gg H_{\mathrm{c} 1}\right)$ (Tinkham 1980). For $0<H<H_{\mathrm{c} 1}$, the microwaves penetrate only the sample surface, while for
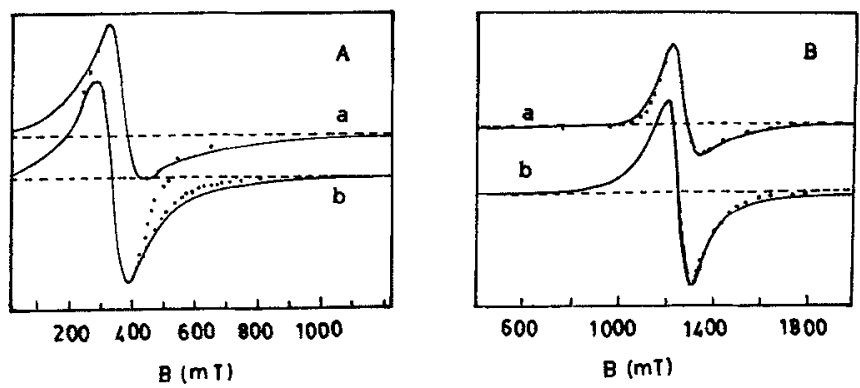

Figure 34. ESR spectra of superconducting, orthorhombic $\mathrm{GdBa}_{2} \mathrm{Cu}_{3} \mathrm{O}_{7}$ (with $T_{\mathrm{c}} \sim 90 \mathrm{~K}$ ), at $300 \mathrm{~K}$. A. (a) X-band $(\nu=9.384 \mathrm{GHz})$ spectrum of single crystal at $20 \mathrm{~mW}$ power and modulation of $2 \mathrm{mT}$ peak-to-peak: $g_{\text {eff }}=1.82$, $\Delta H_{\mathrm{pp}}=128 \pm 6 \mathrm{mT}$ static field parallel to c-axis. The line has been fitted (*) to a Dysonian shape and (b) powder spectrum at $9.380 \mathrm{GHz} g=2.02 \Delta H_{\mathrm{pp}}=112 \pm 6 \mathrm{mT}$, fitted to Lorentzian $\left(^{*}\right)$ and Gaussian (.) shapes. B. (a) $Q$-band $(34.982 \mathrm{GHz})$ spectrum of single crystal at $5 \mathrm{~mW}$ power and $4 \mathrm{mT}$ modulation amplitude. $g_{\text {eff }}=1.96$ and $\Delta H_{\mathrm{pp}}=124 \pm 8 \mathrm{mT}$, fitted to a Dysonian shape $(*)$ and (b) powder spectrum at $35.001 \mathrm{GHz}$ and $5 \mathrm{~mW}$. $g=2.004$ and $\Delta H_{\mathrm{pp}}=108 \pm 4 \mathrm{mT}$ fitted to Lorentzian (*) and Gaussian (.) shapes. In (A) and (B) the ESR intensity of same sample increased by factors of 7 and 20 respectively upon grinding (Deville et al 1993). 
$H>H_{\mathrm{cl}}$ microwaves penetrate into the core of the superconductor. Thus we have a quick and convenient method of determining $H_{\mathrm{cl}}$ of high $T_{\mathrm{c}}$ (type II) superconductor using a conventional EPR spectrometer after compensating for remnant field of the electromagnet (Janes et al 1991).

ESR has been applied to (i) look at the pinning centres for the flux motion in the $\mathrm{YBa}_{2} \mathrm{Cu}_{3} \mathrm{O}_{7}$ superconductor (Baranov and Badalyan 1993), (ii) the nature of the paramagnetic systems in the normal state $\left(T>T_{c}\right)$ of $\mathrm{GdBa}_{2} \mathrm{Cu}_{3} \mathrm{O}_{7}$ (Deville et al 1993) (figure 34) and (iii) the study of flux line lattice through measurement of the magnetic penetration depth $(\lambda)$ in ceramic $\mathrm{YBa}_{2} \mathrm{Cu}_{3} \mathrm{O}_{7}$, $\mathrm{Bi}_{2} \mathrm{Sr}_{2} \mathrm{CaCu}_{2} \mathrm{O}_{8}$ (Rakvin et al 1990), and $\mathrm{Tl}_{2} \mathrm{Ba}_{2} \mathrm{Ca}_{2} \mathrm{Cu}_{3} \mathrm{O}_{10}$ (figure 35) and single crystals of $\mathrm{YB}_{2} \mathrm{Cu}_{3} \mathrm{O}_{7}$ (Koshta et al 1993), with the surfaces coated (decorated) by organic radical of DPPH (the calibration standard for $g$-factor). In the superconducting state, there is observed an inhomogeneously broadened ESR signal from DPPH due to the so-called flux-line lattice formation. From the temperaturedependent linewidth the magnetic penetration depth at $0^{\circ} \mathrm{K}$ $\left(\lambda_{0}\right)$ is calculated. The variation of $\lambda$ follows the law

$$
\left[1-\left(T / T_{\mathrm{c}}\right)^{4}\right]^{-1 / 2}
$$

Experiments on single crystal samples show that the main contribution to inhomogeneous broadening comes from the flux lattice and not from granularity and screening effects.

10.2.3 Fullerenes: Superconductors based on the $\mathrm{C}_{60}$ or Buckminsterfullerene or fullerene, with soccerball structure and unusual electronic structure that makes it accept up to 6 electrons obtained by intercalating it with alkali metals $\mathrm{K}$ (Hebard et al 1991) and $\mathrm{Rb}\left(\mathrm{K}_{3} \mathrm{C}_{60}: T_{\mathrm{c}}=18 \mathrm{~K} ; \mathrm{Rb}_{3} \mathrm{C}_{60}: T_{\mathrm{c}}=28 \mathrm{~K}\right)$, exhibit welldefined ESR spectra in their normal and superconducting states. A precise ESR study of $\mathrm{K}_{x} \mathrm{C}_{60}$ (Kosaka et al 1993) ( $x=3$, face centred cubic (fcc); $x=4$, body centred tetragonal (bct); $x=6$, body centred cubic (bcc)) has clearly identified resonances at $g$ 's $=2.0014,2.0004$ and 1.9952 (figure 36) as arising from the fcc, bct and bcc phases, respectively. In another interesting application to $\mathrm{Rb}_{x} \mathrm{C}_{60}$ compounds (Byszewski et al 1992), thermallytreated samples (diffusion of $\mathrm{Rb}$ into $\mathrm{C}_{60}$ powder at

$\mathrm{Bi}_{2} \mathrm{Sr}_{2} \mathrm{CaCu}_{2} \mathrm{O}_{\mathrm{X}}+\mathrm{DPPH}$
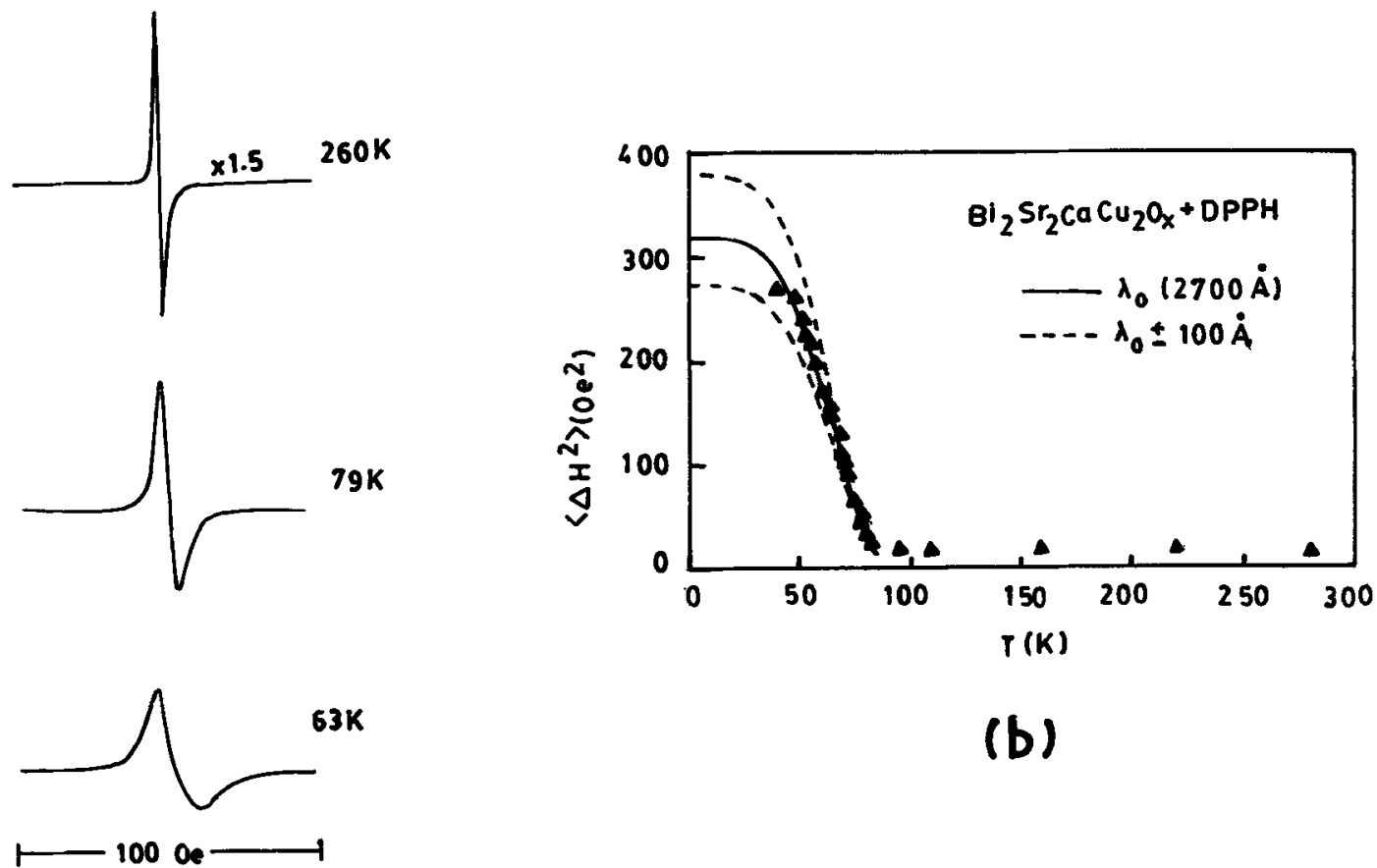

(b)

\section{(a)}

Figure 35. ESR spectra of DPPH absorbed on $\mathrm{Bi}_{2} \mathrm{Sr}_{2} \mathrm{CaCu}_{2} \mathrm{O}_{x}$ superconductor, in order to determine microwave penetration depth. a. Temperature dependence through the superconducting transition temperature $\left(T_{\mathrm{c}}=82 \mathrm{~K}\right)$. Note the significant line broadening below $T_{\mathrm{c}}$ and $\mathrm{b}$. temperature dependence of second moment or linewidth of DPPH ESR signal. The solid line is a theoretical fit (see text) which yields zero temperature penetration depth $\left(\lambda_{0}\right)$ of $2700 \AA$ with $T_{c}=84$. The dashed lines correspond to fits with $2700 \pm 100 \AA$ which shows that the $\lambda_{0}$ deduced is accurate to better than $3.7 \%$ (Rakvin et al 1990). 
$300-450^{\circ} \mathrm{C}$ and subsequent homogenization at 200 $350^{\circ} \mathrm{C}$ ) exhibited ESR signals due to an on-molecule electronic state. Thus clear differences exist in the ESR pattern at the transition from normal to superconducting state, by way of a decrease in ESR signal amplitude at the superconducting to normal state transition. Thus it appears that carriers partially localized on the $\mathrm{C}_{60}$ molecules due to the Coulomb repulsion energy form superconducting states by intermolecular coupling.

\section{Optoelectronic and superionic materials}

\subsection{Optoelectronic materials}

Optoelectronic phenomena rely on the ability of certain materials (e.g. $\mathrm{BaTiO}_{3}, \mathrm{Bi}_{4} \mathrm{Ge}_{3} \mathrm{O}_{12}$ ) to exhibit photochromic and photorefractive effects. These materials respond to light-induced space-charges by large changes in the refractive index (Gunter and Heiguard 1988, 1989). These effects arise from the UV/visible light sensitivity of transition metal impurities doped in these materials. ESR is thus a natural technique to apply to these materials to learn about the valence state of the doping ions, changes in the valence state under illumination, and the physical location of the impurity in the crystal lattice. This basic characterization is crucial for all applications including optical memory and information storage, frequency doubling and parametric oscillation, as an important first step towards optimization of device performance.

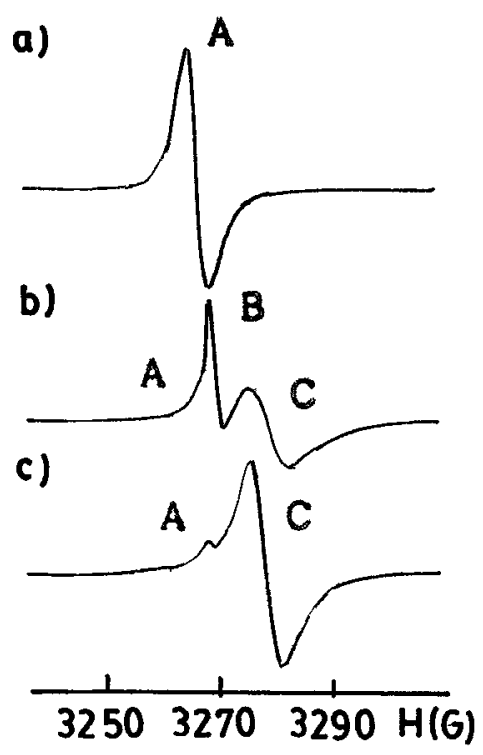

Figure 36. ESR spectra, at $5 \mathrm{~K}$, of $\mathrm{K}$-intercalated $\mathrm{C}_{60}$ (a) $\mathrm{K}_{3} \mathrm{C}_{60}$, (b) $\mathrm{K}_{4} \mathrm{C}_{60}$ and (c) $\mathrm{K}_{6} \mathrm{C}_{60}$. The signals $\mathrm{A}, \mathrm{B}$ and $\mathrm{C}$ correspond to fcc, bct and bcc phases of these fullerenes (Kosaka et al 1993). 11.1a Barium titanate: Barium titanate - the well known ferroelectric material - when doped with transition metal ions of iron group and suitably poled to give wide $(\sim 10 \mathrm{~K})$ thermal hysteresis for the cubic-to-tetragonal phase transitions at nearly $130^{\circ} \mathrm{C}$ on cooling, exhibits high photorefractive gain and is thus of interest for many applications that involve optical phase conjugation and signal processing. The origin of its photorefractive properties i.e. the exact nature of the microscopic centres responsible for the large changes in refractive index light-induced space is linked with (i) control of doping level of single crystals, (ii) discrimination between dopant-related charge-compensation-related effects, and (iii) the oxygen vacancies created by thermal treatments (e.g. in oxidizing/reducing atmospheres).

EPR experiments with transition-metal-doped $\mathrm{BaTiO}_{3}$ powder treated under a wide range of oxygen partial pressures revealed valence state changes in $\mathrm{Co}, \mathrm{Cr}$ and $\mathrm{Mn}$-doped material whereas for the dopants $\mathrm{Fe}, \mathrm{Ni}$ and $\mathrm{Cu}$ such changes were absent, which suggest that the former group of ions are likely to show better photorefractive effect than the latter. Of $\mathrm{Co}, \mathrm{Cr}$ and $\mathrm{Mn}, \mathrm{Mn}$ has a smaller optical absorption while Cr-doped crystals of optical quality are difficult to obtain. Thus Co-doped $\mathrm{BaTiO}_{3}$ emerges as the most promising candidate for photorefraction.

The EPR spectrum of powdered single crystal of $50 \mathrm{ppm}$ Co-doped $\mathrm{BaTiO}_{3}$ processed in a reducing atmosphere at $18 \mathrm{~K}$ (Rytz et al 1990) is shown in figure 37. It exhibits the extended hyperfine structure (by way of two overlapping octets) that arise from the

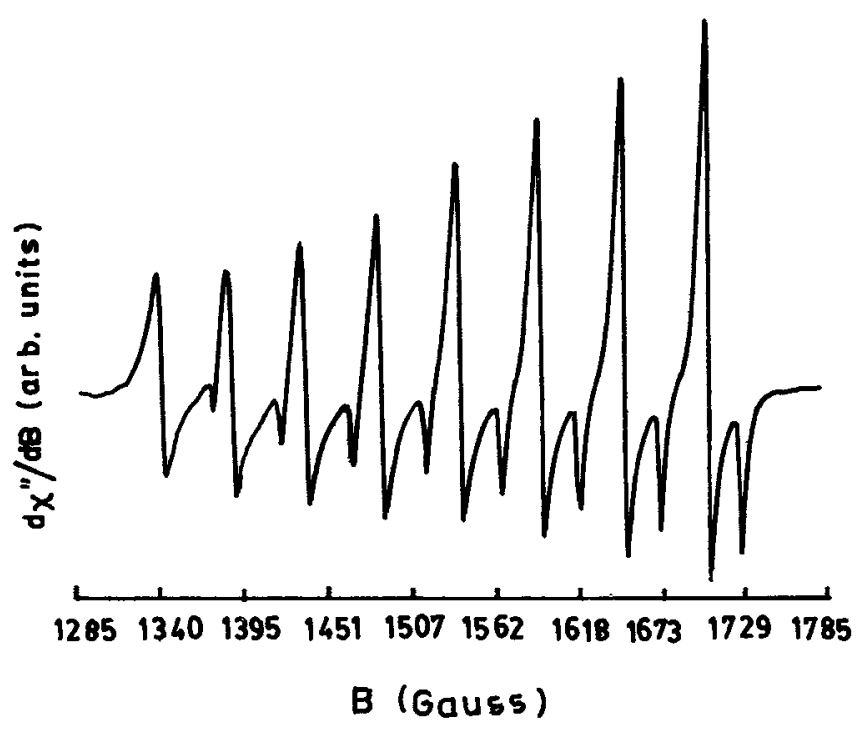

Figure 37. X-band EPR spectrum of powdered single crystal of $\mathrm{BaTiO}_{3}$ : Co at $18 \mathrm{~K}$, recorded at $1 \mathrm{~mW}$ power, and 9.26569 $\mathrm{GHz}$, with a field modulation of $0.2 \mathrm{mT}$. The octet is assigned to $\mathrm{Co}^{2+}$ with $S=3 / 2, I=7 / 2$, with $g_{\|}=g_{\perp}=4 \cdot 341$ and $|A|=5 \cdot 16$ mT (Rytz et al 1990). 
$100 \%$ abundant ${ }^{59} \mathrm{Co}(I=7 / 2)$. For the intense octet, $g_{\|}=g_{\perp}=4.341$ with hyperfine constant $|A|=5.16 \mathrm{mT}$. The octet is assigned to high spin $\mathrm{Co}^{2+}$ with $S=3 / 2$. A spin Hamiltonian with isotropic $g$ and moderate zero field splitting accounts for the spectrum. Besides this, an intense feature with $g=2.004$ due to high spin $\mathrm{Fe}^{3+}$ $(S=5 / 2)$ is also present. This high spin $\mathrm{Co}^{2+}$ not associated with an oxygen vacancy is EPR active, while $\mathrm{Co}^{2+}$, (low spin $S=1 / 2$ ) - oxygen vacancy, complex a product of reduction diamagnetic $\mathrm{Co}^{3+}$ (low spin) - oxygen vacancy complex - though EPR active is not detected at X-band frequencies.

11.1b Lead-zirconate-titanate: Ferroelectric leadzirconate-titanate (PZT) thin films find use in nonvolatile memories and optical information storage devices. Defects and optically-induced metastable trapping centres in PZT control the electrical and optical behaviour. It is thus necessary to understand the nature of these trapping centres.

Warren et al (1993) have identified, through EPR, a positively charged $\mathrm{Pb}^{3+}$ defect centre in PZT $(\mathrm{Zr} / \mathrm{Ti}=53 / 47)$ ceramics. The charged traps were generated by ultraviolet illumination in the band gap region $(3.4 \mathrm{eV})$. The EPR spectrum of the centre, recorded using high power microwave quanta of $0.314652 \mathrm{~cm}^{-1}$ and second harmonic detection, consists of an intense peak with $g=1.995$ at $0.5530 \mathrm{~T}$ arising from nonmagnetic $\mathrm{Pb}$ nuclei, a weaker line $1.145 \mathrm{~T}$, caused by a very large hyperfine $\left(A_{\text {isorropic }}=1.0803 \mathrm{~cm}^{-1}\right.$ or $1.1599 \mathrm{~T}$, $A_{\text {aniso }}=0.00233 \mathrm{~cm}^{-1}$ or $2.5 \mathrm{mT}$ ) with a less abundant ${ }^{207} \mathrm{~Pb}$ nucleus with $I=1 / 2$, on the basis of which the centre is assigned to $\mathrm{Pb}^{3+}$ which has valence-shell configuration of $6 \mathrm{~s}^{1} . \mathrm{Pb}^{3+}$ could arise from some of the $\mathrm{Pb}^{2+}$ corner-sites in the perovskite PZT lattice, by capturing a hole and becoming paramagnetic. The centre is further characterized through its wave function with $40 \%$ 6scharacter and at the most $8 \%$ 6p-character so that there is $48 \%$ localization of unpaired electron on $\mathrm{Pb}$ atom, with the rest of $\approx 52 \%$ unpaired spin density being spread over the 12 oxygen neighbours in the perovskite.

11.1c Bismuth germanate: $\mathrm{Bi}_{4} \mathrm{Ge}_{3} \mathrm{O}_{12}$ has a crystal structure made up of a cubic arrangement of distorted oxygen octahedra surrounding each $\mathrm{Bi}^{3+}$ ion and oxygen tetrahedra around each $\mathrm{Ge}^{4+}$. Thus it offers two sites $\mathrm{Bi}^{3+}$ and $\mathrm{Ge}^{4+}$ - for occupation by a transition metal rare earth impurity ion (Bravo et al 1993). EPR studies have established that $\mathrm{Gd}^{3+}$ impurities occupy $\mathrm{Bi}^{3+}$ sites, while $\mathrm{Cr}$ ions, detected as $\mathrm{Cr}^{4+}$ occupy $\mathrm{Ge}^{4+}$, and $\mathrm{Cr}^{3+}$ occupy $\mathrm{Bi}^{3+}$ sites. $\mathrm{Mn}^{++}$ions are found to occupy $\mathrm{Bi}^{3+}$ sites without charge compensation, and undergo change of valence under UV illumination. $\mathrm{Co}^{2+}$ ions, likewise, are found to occupy $\mathrm{Bi}^{3+}$ probably without charge compensation. 11.1d Lithium vanadate and lithium borate: These are nonlinear optical materials employed for doubling of optical frequencies e.g. to obtain blue/green light from an infrared laser beam. These materials need to be thoroughly characterized for trace chemical impurities and for irradiation-induced defects because their optical and electrical properties are strongly influenced by defects and impurities.

Lithium vanadate $\left(\mathrm{Li}_{3} \mathrm{VO}_{4}\right)$ when prepared in $\beta$-II phase, has a second harmonic generation behaviour comparable to that of $\mathrm{LiNbO}_{3}$ (Sakita and Fujii 1991). Two ESR active, trapped hole centres are formed when $\mathrm{Li}_{3} \mathrm{VO}_{4}$ is exposed to X-rays at $77 \mathrm{~K}$ (Murata and Miki 1993): (i) $\mathrm{CO}_{3}^{-}$, stable at ambient, with $g_{1}=2.021, g_{2}=2.011$ and $g_{3}=2.0057$, coming from $\mathrm{Li}_{2} \mathrm{CO}_{3}$ used in sample preparation, and (ii) an intrinsic $\mathrm{O}^{-}$type centre with one neighbouring vanadium ion, with $g_{x x}=g_{y y}=2.026$, $g_{z x}=2.028$ and $A_{x x}=A_{y y}=2.05 \mathrm{mT}, A_{z z}=3.075 \mathrm{mT}$ due to ${ }^{51} \mathrm{~V}(I=7 / 2)$ nucleus. This second centre is unstable above $150 \mathrm{~K}$ and is completely annealed at room temperature. Two significant features of this centre viz. poor thermal stability and no coloration at ambient temperature favour the use of $\mathrm{Li}_{3} \mathrm{VO}_{4}$ as a frequency doubler.

$\mathrm{LiB}_{3} \mathrm{O}_{5}$ is a common second-harmonic generator material, involving a fundamental wave, around $1.06 \mu \mathrm{m}$, with a phase-matching angle along a crystallographic axis. Two unique properties: (i) temperature dependent refractive indices, and (ii) wide (160-1300 nm) range of optical transmission make this an ideal nonlinear material.

Two prominent point defects: (i) a trapped hole centre localized on an oxygen ion near $a{ }^{11} B$ nucleus (with a smaller "B hyperfine structure), and (ii) a trapped electron centre - also localized on a "B but with a larger hyperfine structure - have been observed in the ESR and ENDOR of this biaxial crystal irradiated with $\gamma$-rays near $77 \mathrm{~K}$ (Scripsick 1993). The significant findings are: (i) the lack of ESR spectra due to transition metal impurities $\mathrm{Fe}^{3+}, \mathrm{Cr}^{3+}$ and $\mathrm{Mn}^{2+}$ present at $<100 \mathrm{ppb}$ level in $\mathrm{LiB}_{2} \mathrm{O}_{5}$ rather than at ppm level in other SHG materials $\mathrm{LiNbO}_{3}$ and $\mathrm{KTiOPO}_{4}$, and (ii) both the irradiationinduced centres thermally anneal in the $120-130 \mathrm{~K}$ range.

11.1e Other materials: A recent EPR study has demonstrated (Whitmore 1993) that tetrahedral $\mathrm{Cr}^{4+}$ is responsible for the near-infrared laser activity in $\mathrm{Cr}$-doped Forsterite $\left(\mathrm{Mg}_{2} \mathrm{SiO}_{4}\right) . \mathrm{Fe}^{3+}, \mathrm{Fe}^{3+}$-oxygen vacancy, $\mathrm{Co}^{2+}$, $\mathrm{Co}^{2+}$-oxygen vacancy and $\mathrm{Ir}^{4+}$ have been identified in photorefractive $\mathrm{KNbO}_{3}$ in its rhombohedral and orthorhombic phases (Possenriede 1989).

\subsection{Superionic materials}

Superionic conductors are ionic solids whose conductivities at ambient temperatures are of the order of molten salt electrolytes and thus they are also called solid 
electrolytes. Thus any application of ESR to such systems must aim at elucidating the effects of ionic motion and to determine site symmetries of defects that block favoured hopping paths in the superionic conductor.

Four such studies are noteworthy: (1) $\mathrm{Mn}^{++}$in $\mathrm{PbF}_{2}$, (2) $\mathrm{Cu}^{++}$in $\beta$-sodium gallate, (3) $\mathrm{Ag}^{++}$in $\beta$-alumina and (4) $\mathrm{Ag}^{++}$in $\mathrm{AgI}-\mathrm{Ag}_{2} \mathrm{O}-\mathrm{B}_{2} \mathrm{O}_{3}$ glass.

11.2a Mn-doped lead fluoride: $\mathrm{PbF}_{2}$ is an $\mathrm{F}^{-}$ion conductor at high temperatures where the high mobility of $\mathrm{F}^{-}$ions is expected to affect the EPR of $\mathrm{Mn}^{++}$ions by way of line narrowing, line broadening and disappearance of additional structure in each of the $\mathrm{Mn}^{++}(I=5 / 2)$ hyperfine components (Evora and Jaccorino 1977) due to $F$ nuclei observed at low temperatures (figure 38 ). Indeed, at the temperatures of onset of motional narrowing $\left(220^{\circ} \mathrm{C}\left({ }^{19} \mathrm{~F}\right.\right.$ hf frequency)) $\tau_{\text {conductivity }} \sim 1$. Above $400^{\circ} \mathrm{C}$, the lines start to broaden due to a fluctuating crystal field. The linewidth increases as $m_{1}^{2}$, where $m_{1}$ is the nuclear magnetic quantum number, which is interpreted as acoustic phonon enhanced spectral density of the fluctuating crystal field. $\mathrm{F}^{-}$sublattice melting enhances this interaction. This phonon coupling is greatly enhanced by rapid ionic diffusion, giving lines as narrow as $0.02 \mathrm{mT}$.

$11.2 \mathrm{~b}$ Copper-doped $\beta$-sodium gallate: In a study of $\mathrm{Cu}$-doped single crystal $\beta$-sodium gallate isostructural with $\beta$-alumina, and with nearly same $\mathrm{Na}^{+}$conductivity it was found that at room temperature, the anisotropic hyperfine structure of ${ }^{63.65} \mathrm{Cu}(l=3 / 2)$ and the $g$-anisotropy of $\mathrm{Cu}^{++}$resonance is washed out/averaged out due to local symmetry fluctuations through $\mathrm{Cu}^{++} /$ligand motion

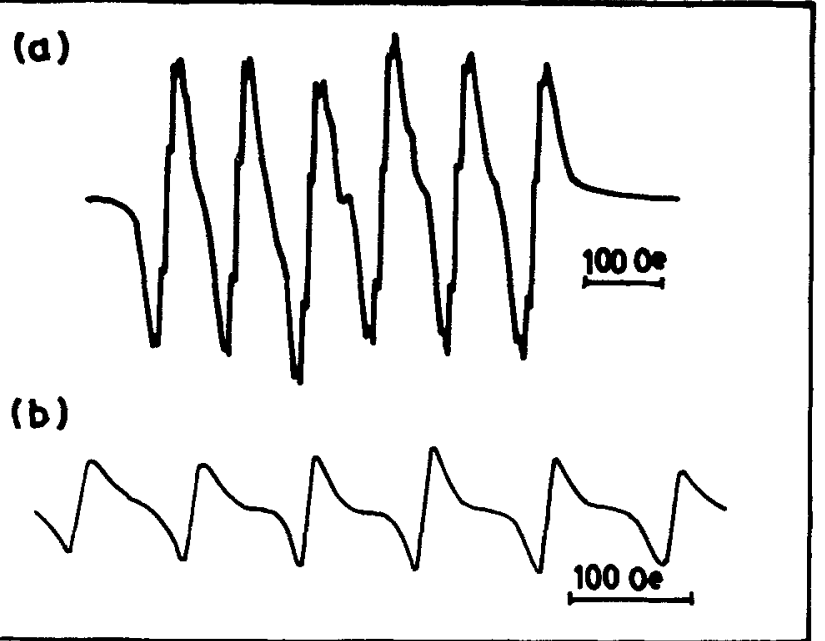

Figure 38. EPR spectra of $\mathrm{Mn}^{++}$in $\mathrm{PbF}_{2}$ at (a) $77 \mathrm{~K}$ and (b) $660 \mathrm{~K}$. Note the line broadening and absence of additional structure in (b) when $\mathrm{PbF}_{2}$ is in the disordered superionic phase (Evora and Jaccorino 1977).
(Title and Chandrasekhar 1976). A correlation time (for the $\mathrm{Cu}^{++} / \mathrm{Cu}-\mathrm{O}$ complex) of $10^{-11} \mathrm{sec}$, two orders faster than $\mathrm{Na}^{+}$hopping time $\left(10^{-9} \mathrm{sec}\right)$ was deduced. A motional effect correlating with ionic diffusion is involved, whereby rapid fluctuations of bridging $\mathrm{O}^{2-}$ ions with respect to $\mathrm{Cu}^{++}$triggered by rapid $\mathrm{Na}^{+}$diffusion could give rise to the isotropic, structureless ESR line observed.

11.2c $\mathrm{Ag}$ centres in $\beta$-alumina: Atomic $\mathrm{Ag}^{0}$ centres and hole $\mathrm{Ag}^{2+}$ centres were detected in $\mathrm{X}$-irradiated (77 K) $\beta$-alumina crystals (Badalyan and Zhitnikov 1985). $\mathrm{Ag}$ was incorporated by immersing $\mathrm{Na}^{+} \beta$-alumina crystals in molten $\mathrm{AgNO}_{2}$ at $350^{\circ} \mathrm{C}$. Orientation dependence of $g$-factor revealed that $\mathrm{Ag}^{2+}$ becomes stabilized at $77 \mathrm{~K}$ in a position between two oxygen ions as a minor plane. It is this minor plane that separates the four alternate spinel blocks (containing $\mathrm{Ag}$ and $\mathrm{O}$ ions) of the $\beta$-alumina (11 $\mathrm{Al}_{2} \mathrm{O}_{3} \cdot \mathrm{Na}_{2} \mathrm{O}$ ) structure, and that contains the mobile $\mathrm{Na}^{+}$ions besides $\mathrm{O}$ ions, and it is these $\mathrm{Na}^{+}$ions that are replaced by $\mathrm{Ag}^{+}$ions. Two environments for $\mathrm{Ag}^{2+}$ are indicated, with the same axial $g$ but with two different sets of planar $g$ 's. The ${ }^{107.109} \mathrm{Ag}$ hyperfine interaction constants are low, suggestive of a strong delocalization of the hole from $\mathrm{Ag}^{\text {t+ }}$ ion to two oxygen ions on the axis of the centre. $\mathrm{A}$ model for $\mathrm{Ag}^{++}$based on these results is shown in figure 39.

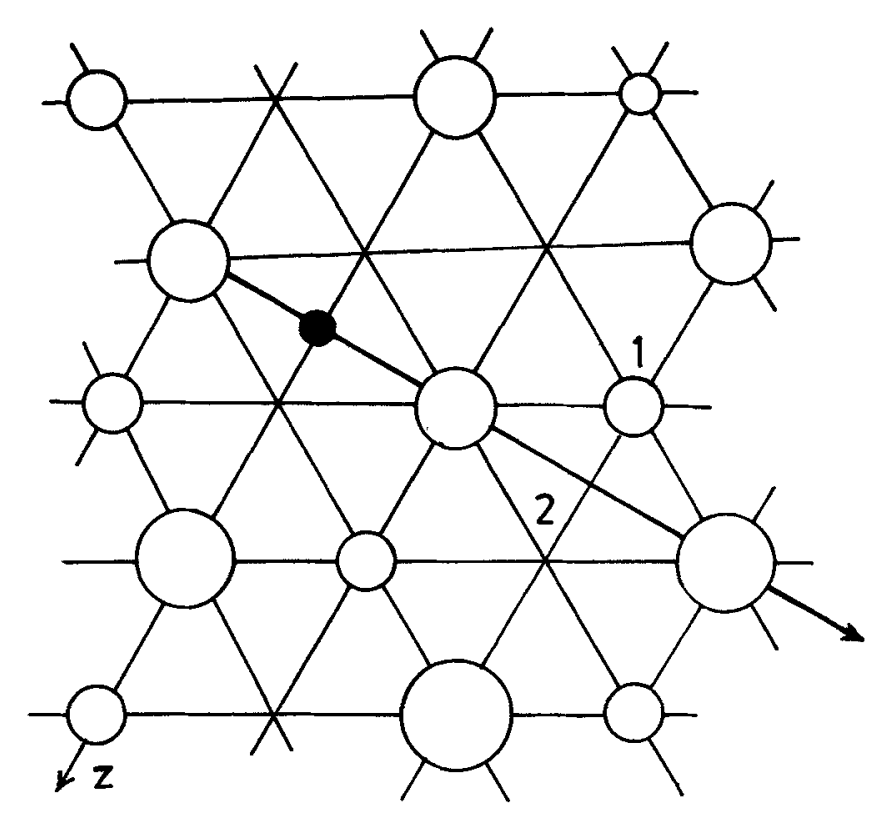

Figure 39. Schematic representation of a reflection plane in the hexagonal $\beta$-alumina $\left(\mathrm{Na}_{2} \mathrm{O} \quad 11 \quad \mathrm{Al}_{2} \mathrm{O}_{3}\right)$ crystal containing $\mathrm{Ag}^{+}$ions showing the position occupied by $\mathrm{Ag}^{++}$centre formed by $\mathrm{X}$-irradiation (dark circle). Large circles are $\mathrm{O}^{2-}$ and small ones $\mathrm{Ag}^{+} / \mathrm{Na}^{+}$ions. 1 and 2 are the occupied and vacant cation sites called Beevers-Ross and anti-Beevers-Ross sites. In the superionic state, $\mathrm{Ag}^{+} / \mathrm{Na}^{+}$ions spend some time at 2 (Badalyan and Zhitnikov 1985). 
11.2d $\mathrm{Ag}^{++}$in $\mathrm{AgI}-\mathrm{Ag}_{2} \mathrm{O}-\mathrm{B}_{2} \mathrm{O}_{3}$ glass: ESR-active $\mathrm{Ag}^{++}$ ions, stabilized during melt-quenching, in the optimum conductivity $60 \mathrm{AgI}-30 \quad \mathrm{Ag}_{2} \mathrm{O}-10 \mathrm{~B}_{2} \mathrm{O}_{3}$ glass have been used to probe the glass structure and $\mathrm{Ag}^{+}$conduction mechanism (Balaya and Sunandana 1990). Two clearly distinguishable $\mathrm{Ag}^{++}$ESR species with different thermal stabilities were identified (figure 40, table 13) and attributed $\mathrm{Ag}^{++}$in different chemical surroundings. The ESR data neatly correlate with Minami's structural model (Minami et al 1982) for this glass and $\mathrm{Ag}^{+}$conductivity. The centre $\mathrm{I}$, with a smaller $A_{\perp}$ due to $(I=1 / 2$ of $\mathrm{Ag})$ is due to less mobile $\mathrm{Ag}^{2+}$ bonded covalently to nonbridging oxygens of $\mathrm{BO}_{3}$, and thus thermally more stable, and not involved in conduction. While the centre II with a larger $A_{\curlywedge}$, should arise from $\mathrm{Ag}^{+}$ion ionically bonded to bridging oxygens of $\mathrm{BO}_{4}$ groups in the borate glass network, are more mobile and contribute to $\mathrm{Ag}^{+}$conduction. $\mathrm{Ag}^{2+}$ ESR has also been observed in $\mathrm{Ag}_{2} \mathrm{O}-\mathrm{TeO}_{2}$ (Balaya and Sunandana 1992) glasses where the large covalency of $\mathrm{Te}-\mathrm{O}$ bond in the glass structure leads to washing out of Ag-hyperfine structure in the ESR spectra.

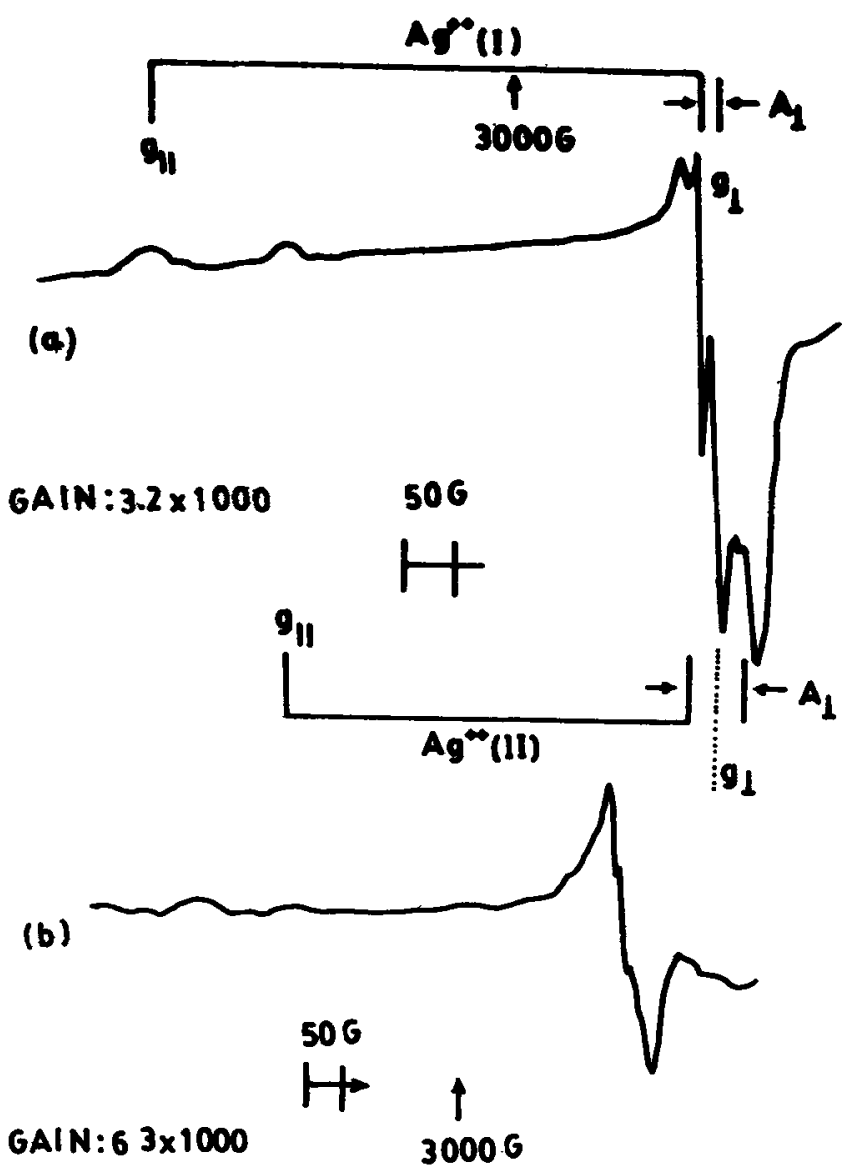

Figure 40. EPR spectra of $\mathrm{Ag}^{2+}$ in the superionic conducting $60 \mathrm{AgI} \cdot 30 \mathrm{Ag} 2 \mathrm{O} \cdot 10 \mathrm{~B}_{2} \mathrm{O}_{3}$ glass at (a) $303 \mathrm{~K}$ and (b) $413 \mathrm{~K}$. Note that of the two centres $\mathrm{Ag}^{++}$(I) and $\mathrm{Ag}^{++}$(II), the first one is thermally more stable, and is thus less mobile, while the second one contributes significantly to conductivity (see text) (Balaya and Sunandana 1990). 11.2e Other materials: ESR of $\mathrm{CO}_{3}^{-}$radical formed by $\mathrm{X}$-irradiation in $\mathrm{CO}_{3}^{2-}$ doped $\mathrm{Li}_{2} \mathrm{SO}_{4} \cdot \mathrm{H}_{2} \mathrm{O}$ single crystal (monoclinic) and cubic (fcc) $\mathrm{Li}_{2} \mathrm{SO}_{4}$ stabilized at ambient by programmed quenching from the melt aided by a few mole $\% \mathrm{Li}_{2} \mathrm{CO}_{3}$ has been used as a structure sensitive probe (Balaya and Sunandana 1990). The high conducting cubic phase is characterized by a nearly isotropic ESR signal with $g=2.0094$ and $\Delta H_{\mathrm{pp}}=0.6 \mathrm{mT}$ resulting from motional averaging of $\mathrm{CO}_{3}^{-}$ions. ESR of DPPH adsorbed on AgI has been used to probe the hexagonal-cubic phase transition $\left(147^{\circ} \mathrm{C}\right.$ ) in $\mathrm{AgI}$ (Murthy and Sunandana 1992).

\section{ESR imaging and microscopy}

\subsection{ESR imaging}

Imaging of the spatial distribution of the properties of advanced materials, especially on a microscopic scale enables a deeper insight into their (physico-chemical) nature to be obtained. ESR imaging (Ikeya 1991) allows for an accurate mapping of the spatially distributed paramagnetic species in the sample. In a typical continuous-wave magnetic resonance imaging experiment, the material is initially placed in a highly uniform dc magnetic field and its ESR spectrum measured (Lauterbur 1973). Then a small magnetic field gradient $(1-10 \mathrm{mT} / \mathrm{cm})$ is applied external to the microwave carrier (or inside it) and the broadened, convoluted ESR spectra is recorded. Deconvolution, with the original ESR spectral lineshape gives the spatial distribution of spins because the magnetic field intensity corresponds to the position under a linear magnetic field gradient.

Suppose small magnetic field gradients $\partial H / \mathrm{d} x, \partial H / \mathrm{d} y$ and $\partial H / \mathrm{d} z$ are applied, through properly positioned field coils, along $x, y$ and $z$ directions with the uniform field $\left(H_{0}\right)$ along $z$-direction $\left(H_{0}\right)$. Then the field at the spatial site $(x, y, z)$ is given by

$$
H(x, y, z)=H_{0}+(\partial H / \partial x) x+(\partial H / \partial y) y+(\partial H / \partial z) .
$$

Resonance occurs at the magnetic field $H_{r}$, for the microwave frequency $\nu$, when $h v=g \mu_{\mathrm{B}} H_{\mathrm{r}}$. Thus the field intensity obtained by sweeping $H_{0}$ is indicative of the positions.

The spatial distribution function of the spins being imaged, $f(z)$ can be expressed as a function $f(H)$ of the magnetic field $H$ because

$$
H=H_{0}+(\partial H / \partial z) z=H_{0}+b z .
$$

The ESR spectrum under the field gradient, $g(H)$ is then expressed as a convolution of $f(H)$ and the signal shape function $r(H)$ in the uniform magnetic field as:

$$
g(H)=\int_{0}^{\infty} r\left(H-H^{1}\right) f(H) \mathrm{d} H .
$$


Then the Fourier transform (FT) of the above integral is obtained as

$$
G(\omega)=R(\omega) F(\omega),
$$

where $G(\omega), R(\omega)$ and $F(\omega)$ are the $\mathrm{FT}^{s}$ of $g(H), r(H)$ and $f(H)$, respectively. Finally the distribution function $f(H)$ may be obtained by deconvolution using the inverse FT of $F(\omega)=G(\omega) / R(\omega)$ with same filter function as $R(\omega)^{-1}$ diverges.

There are advantages of modulating the field gradient.

The resolution of ESR imaging is limited by the linewidth of the resonance signals and the available field gradients.

12.1a Use of a linear field gradient: By using a straight four-wire configuration inside a cavity, Furusawa and Ikeya (1991) have imaged an irradiated teflon tube. The linear gradient coil system in a $\mathrm{TE}_{011}$ cavity is shown in figure 41 . The four copper wires connected to each other and to current sources constitute four independent current loops, the current magnitude and direction of each of which is controlled to produce the linear gradient. Thus the current through the wire located at $(\gamma, \theta)$ in the $y-z$ plane is controlled by the equation

$$
I(\theta)=I_{\max } \cos (\theta-\phi),
$$

where $r$ is the radius of the cylinder, $\theta$ the angle from $y$-axis, $I_{\max }$ the maximum current per wire, $\phi$ the direction of the linear field gradient produced. The magnetic field around the centre of the four-wire line on gradient wire is

$$
B_{z}(y, z)=\frac{4 \mu_{0} I_{\max }}{\pi \cdot r^{2}}(y \cos \phi+z \sin \phi),
$$

(where $\mu_{0}$ is the magnetic permeability of the free space) which implies that the field gradient makes an angle $\phi$ with $y$-axis and has a magnitude $8 \mu_{0} I_{\max } / \pi r^{2}$ (Furusawa and Ikeya 1991). The deconvolution process, the important intermediate step to imaging is shown in figure 42 for the case of a $\gamma$-irradiated teflon tube. The ESR spectrum of this sample, without a field gradient (figure 42a) is used as an instrumental function to obtain the deconvoluted spectrum (figure 42c) of the ESR spectrum recorded with field gradient of $1 \mathrm{~T} / \mathrm{m}$ (figure $42 \mathrm{~b}$ ). The ESR image is finally obtained using the filtered back projection technique (Ohno 1982).

12.1b Use of two-dimensional wire arrays: Using an ordinary commercial ESR spectrometer, Ikeya et al (1991) have used two-dimensional wire arrays $(i \times j)$ for scanning the local static (DC) field and the modulation $(100 \mathrm{kHz} . \mathrm{AC}$ ) field (figure 43a). These two fields - DC and $\mathrm{AC}$ - are electronically scanned by switching the current through the array. The spectra for the $\mathrm{AC}$ and DC currents at various locations of the $i \times j$ array are shown schematically in figure $43 \mathrm{~b}$. When the test sample (DPPH) is outside the loop current from $i$ th to $(i+1)$ th wires, an out-of-phase derivative signal is obtained. This is an example of a two-dimensional imaging which when obtained as a plot of signal intensities gives the distribution of spins on the sample surface.

A spectral-spatial two-dimensional imaging of $E^{\prime}$ defects in X-irradiated $\mathrm{SiO}_{2}$ has been performed by Sukei et al (1993) using a Varian E.9 X-band spectrometer and an additional set of coils which give a gradient of $10 \mathrm{mT} / \mathrm{cm} / \mathrm{amp}$ along the main magnetic field and a computer for data collection and iterative image recon-

\begin{tabular}{|c|c|c|c|c|}
\hline \multirow[b]{2}{*}{ Sample } & \multicolumn{2}{|c|}{$g$} & \multicolumn{2}{|c|}{$A$} \\
\hline & $g_{\|}$ & $g_{\perp}$ & $A_{\|}(\mathrm{mT})$ & $A_{\perp}(\mathrm{mT})$ \\
\hline $\begin{array}{l}60 \mathrm{AgI}-30 \mathrm{Ag}_{2} \mathrm{O}-10 \mathrm{~B}_{2} \mathrm{O}_{3} \\
\text { Centre I } \\
\text { Centre II }\end{array}$ & $\begin{array}{l}2 \cdot 495(2) \\
2 \cdot 373(2)\end{array}$ & $\begin{array}{l}2.060(2) \\
2.054(2)\end{array}$ & $\begin{array}{l}\text { not resolved } \\
\text { not resolved }\end{array}$ & $\begin{array}{l}1 \cdot 8(0 \cdot 3) \\
5 \cdot 6(0 \cdot 3)\end{array}$ \\
\hline $\begin{array}{l}30 \mathrm{Ag}_{2} \mathrm{O}-70 \mathrm{~B}_{2} \mathrm{O}_{3} \\
\text { Centre I } \\
\text { Centre II }\end{array}$ & $\begin{array}{l}2 \cdot 503(2) \\
2 \cdot 388(2)\end{array}$ & $\begin{array}{l}2.065(2) \\
2.059(2)\end{array}$ & $\begin{array}{l}\text { not resolved } \\
\text { not resolved }\end{array}$ & $\begin{array}{l}2 \cdot 0(3 \cdot 0) \\
6.5(0.3)\end{array}$ \\
\hline $\begin{array}{l}30 \mathrm{Ag}_{2} \mathrm{O}-70 \mathrm{TeO}_{2} \\
\text { Centre I } \\
\text { Centre II }\end{array}$ & $\begin{array}{l}2 \cdot 493(2) \\
2 \cdot 389(2)\end{array}$ & 2.070 & $\begin{array}{l}\text { not resolved } \\
\text { not resolved }\end{array}$ & $\begin{array}{l}\text { not resolved } \\
\text { not resolved }\end{array}$ \\
\hline $\begin{array}{l}\mathrm{Ag}_{2} \mathrm{O}-\mathrm{B}_{2} \mathrm{O}_{3} \\
\mathrm{X} \text {-irradiated }\end{array}$ & $2 \cdot 310$ & $2 \cdot 040$ & & \\
\hline $\begin{array}{l}\text { Silver activated phosphate glass } \\
\mathrm{X} \text {-irradiated at } 300 \mathrm{~K}\end{array}$ & $2 \cdot 350$ & 2.050 & not resolved & not resolved \\
\hline
\end{tabular}
struction with filtered back projection.

Table 13. $\mathrm{Ag}^{2+}$ ESR parameters in silver iodo-borate and related glasses*.

*Adapted from Balaya P 1992 Electrical, thermal and spectroscopic studies on disordered superionic conductors, Ph.D. thesis, University of Hyderabad, Hyderabad. 


\subsection{ESR microscopy}

A one-dimensional scanning ESR microscope based on a microwire array has been built by Miyamura and Ikeya (1993) to obtain ESR images of fossils. This instrument (figure 44a) involves scanning of the localized magnetic field moderation (instead of the static magnetic field) which is facilitated by placing the microwire array on the sample holder. The modulated ESR signals are detected by lock-in-amplifier, ensuring high-sensitive signal detection.

The images of a 'point' DPPH powder (figure 44b) and a naturally irradiated fossil shark tooth, containing

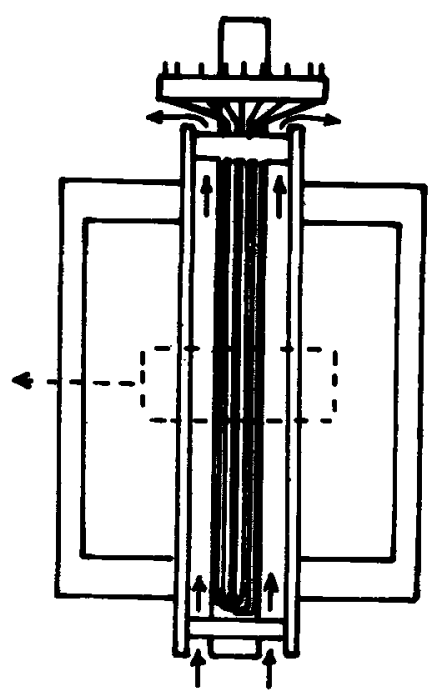

quartz tube
(inner)

stopper

cavity (TE 011 )

gradient coil

quartz tube (outer)

magnetic field cooling gas

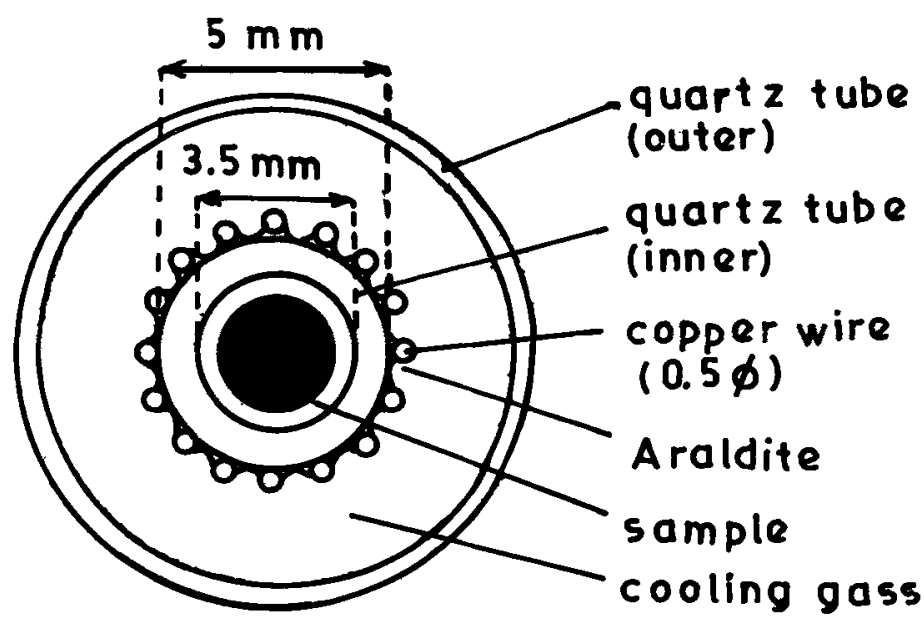

Figure 41. The use of a linear field gradient in ESR imaging. a. Field gradient coil in $T_{011}$ cylindrical cavity and $\mathbf{b}$. cross section of the central portion of the gradient coil in the $y-z$ plane (Furusawa and Ikeya 1991).
$\mathrm{CO}_{2}^{-}$radical detected at $g=2.0025$ and $g=1.998$. The signal intensities is high at the enamel point of the tooth at both edges (figure 44c).

Important emerging applications of ESR microscopy include the following (Ikeya 1991):

(I) Monitoring of distribution of active species during catalytic reactions to clarify mechanisms and to design large catalytic reactors.

(II) Evaluation of crystal perfection using anisotropic ESR signals.

(III) Information on crystal growth and inner mantle of the earth, using images of synthetic ruby/sapphire and natural diamonds, respectively.

\section{Emerging techniques}

Apart from the techniques discussed in $\S 4$, which are routinely employed for the characterization of advanced materials, several new innovative techniques have been developed during the last decade. In what follows a few of these are briefly discussed and possible application areas mentioned.

\subsection{Loop gap resonators and multi frequency EPR}

The cavity resonators employed in EPR spectrometers (e.g. $\mathrm{TE}_{0,1}$ cylindrical cavity) are distributed circuits, and
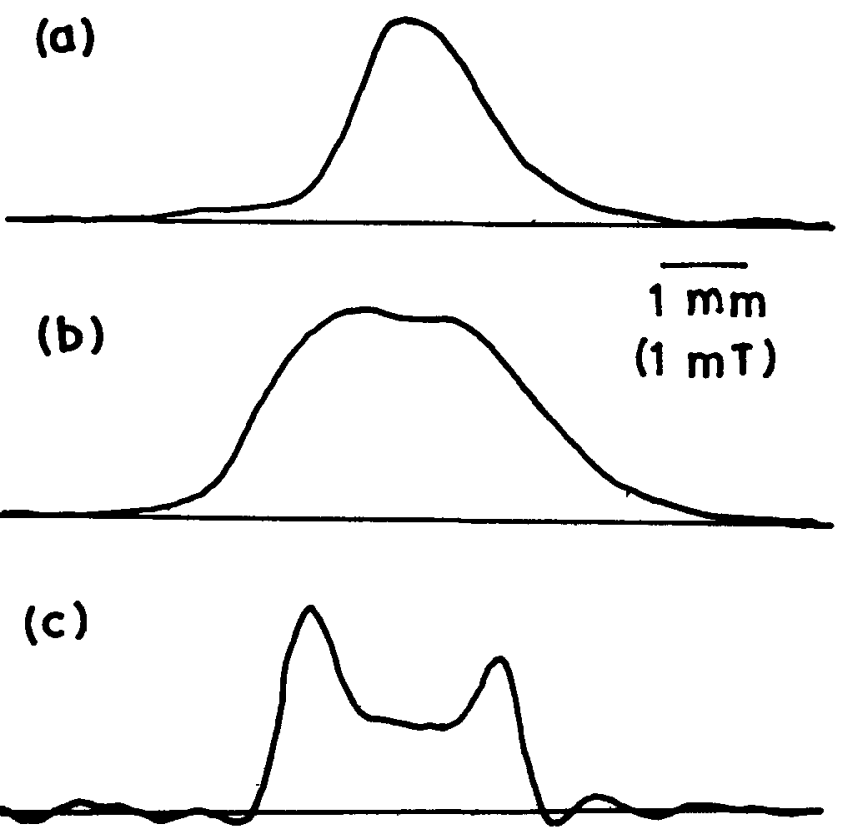

Figure 42. ESR spectrum of a $\gamma$-irradiated teflon tube. a. Without a magnetic field gradient, $b$. with a field gradient of 1 $\mathrm{T} / \mathrm{m}$ and $\mathbf{c}$. after deconvolution of $\mathbf{b}$ using spectrum a as an instrumental function (Furusawa and Ikeya 1991). 


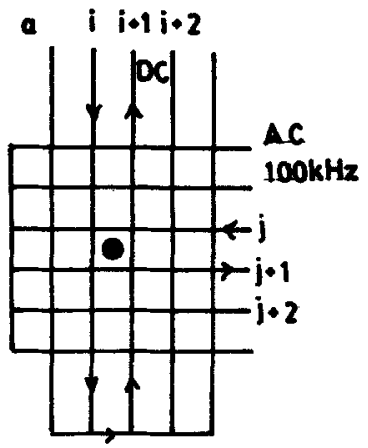

2-D Microwire Arrays DC-AC 2-D Microscope

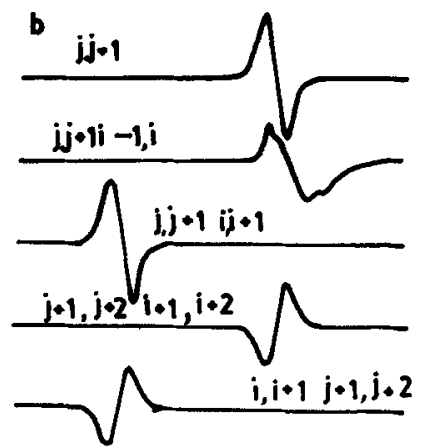

$$
H_{0}-\Delta H_{1} \quad H_{0}
$$

Figure 43. Two-dimensional ESR imaging. a. Wire arrays $(i \times j)$ for scanning the static $(\mathrm{dc})$ field and the modulation (ac) field and $b$. spectra for ac and dc currents at selected locations of the wire array with ac current at $j$ th and $(j+1)$ th wires and dc current at $j$ th wires as indicated (Ikeya et al 1991). the dimensions of these cavities are of the order as the wavelength of the microwave radiation. The electric and magnetic field vectors are interdependent and related by Maxwell's equations. The loop gap resonator or LGR, on the other hand, is based on the lumped circuit concept, with the circuit elements $R, L$ and $C$ are clearly defined, the circuit dimensions being small compared to the wavelength by typically $1 / 10$ to $1 / 3$. Most importantly, the electric and magnetic fields are independent of each other in the lumped circuit limit.

For a simple loop of radius $r$ with $n$ gaps, each of width $W$, separated by $t$ units, the inductance is given hy $(n=1,2,4)$

$$
L=\frac{\mu_{0} \pi r^{2}}{Z}
$$

and the capacitance by

$$
C=\frac{\varepsilon W Z}{t n},
$$

where $\mu_{0}$ and $\varepsilon$ are free space permittivity and dielectric

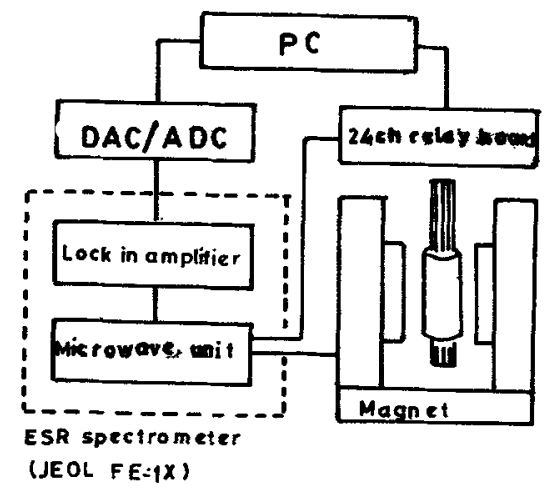

(a)

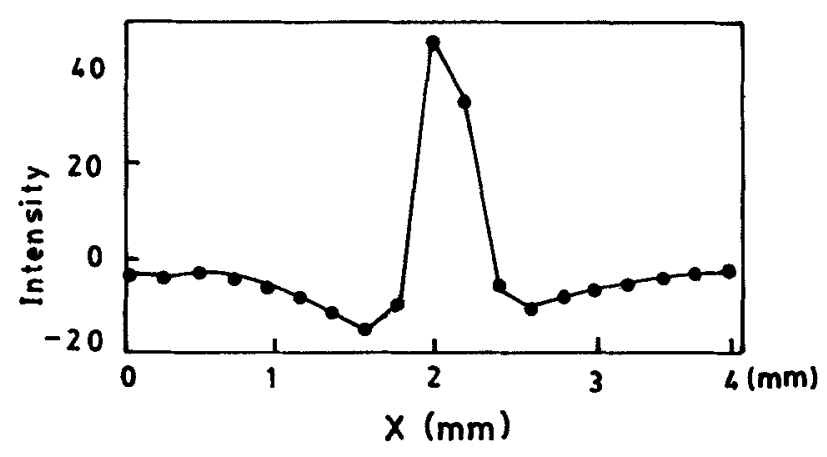

(b)

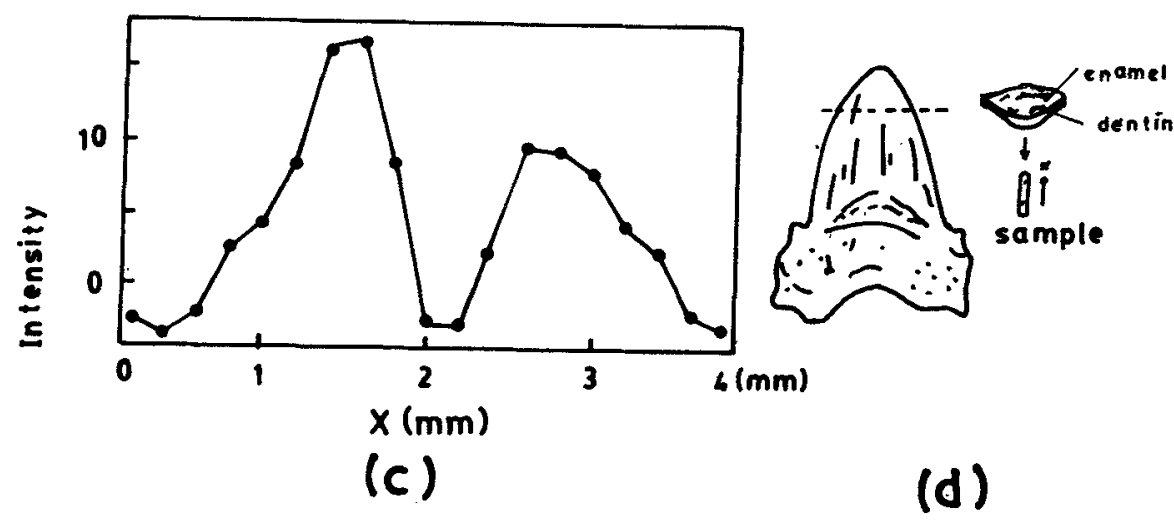

Figure 44. The scanning ESR microscope with micro-wire array and its applications. a. Bloch diagram, b. ESR intensity distribution for a DPPH point sample (powder), $200 \mu \mathrm{m}$ diameter and c. one-dimensional ESR image of a fossil shark tooth, cut and scanned as illustrated (Miyamura and Ikeya 1993). 
constant and $Z$ the impedance of the circuit. The resonance frequency is

$$
v_{\mathrm{r}}=\frac{1}{2 \pi \sqrt{L C}}
$$

An X-band LGR has a central loop $1-3 \mathrm{~cm}$ in diameter, which contains the sample whose EPR spectrum is required. The structure is shielded by a $\sim 2 \mathrm{~cm}$ diameter radiation shield. The filling factor is nearly 1 for a sample in such a loop. Generally, LGR's have been constructed from low frequencies $(10 \mathrm{MHz}$, for magnetic resonance imaging) to $35 \mathrm{GHz}$ and Q's of $500-2000$ have been realized.

An important technical advantage of LGR is the possibility of doing multifrequency EPR i.e. the capability of examining the same sample over a range of frequencies say from 0.5 to 1 and from 4 to $8 \mathrm{GHz}$ (Hyde and Froncisz 1989). For pulsed EPR investigation at X-band frequencies a bridged LGR (BLGR) has been designed (Pfenninger et al 1988). With its high rf transparency BLGR is suitable for a variety of multifrequency experiments including pulsed and hyperfineselective ENDOR, and, Fourier transform EPR (Schweiger 1989).

\subsection{Millimeter wave EPR}

Synthesis and processing of signals in the far-infrared and millimeter wave regions for applications in radar, communications and radio astronomy, have stimulated the efforts towards high frequency and high field EPR spectroscopy. Specific advantages of this technique are increased spectral resolution, permitting very accurate determination of $g$-tensor principal values, and increased sensitivity to molecular motions besides increased absolute sensitivity for detection (number of electron spins/unit field). Freed and coworkers (1989) have built an $1 \mathrm{~mm}$ wave EPR spectrometer that operates at $249.9 \mathrm{GHz}$ generated by a solid state (an InP Gunn Oscillator) and $8.9 \mathrm{~T}$ (generated by a superconducting magnet) for $g \sim 2$, with a sensitivity comparable to that of a $9 \mathrm{GHz}$ EPR spectrometer. Their design is based on far-infrared technology that uses the principles of Gaussian optics to propagate the millimeter waves, 'feedhorns' being used for launching the beam and to convert it into a Gaussian beam, and couple it back to the Fabry-Perot cavity resonator housing the sample in a teflon holder. The detector is a Schottky diode coupled to a low-noise video amplifier, whose response is amplified by a lock-in-amplifier, to be digitized and fed to a personal computer.

An important application of this spectrometer is based on its ability to resolve $g$-anisotropy of nitroxide spin labels and organic free radicals such as DPPH.

\subsection{Reaction yield detected magnetic resonance (RYDMR)}

Many photophysical processes, such as the rate of recombinations of excess carriers in semiconductors, involve the formation of very short-lived ( $\leq$ ten nanosec) paramagnetic states, which are not detectable either by continuous wave EPR ( microsecond time scale) or pulsed EPR/spin echo techniques (tens of nanosecond resolution). The RYDMR experiment on the other hand, monitors the product that is usually much longer lived than the paramagnetic precursor state but detects it through an easily measured property e.g. its optical absorption. The experiment (Lersch and Michel-Beyerle 1989), as in time-resolved EPR, consists in irradiating resonant microwaves on to the sample during the lifetime of the paramagnetic state of interest. The effect of microwave irradiation is detected as a change of the product yield of some spin-selective reaction of the spincorrelated pairs. The virtue of the RYDMR technique is the effective decoupling of the stages of inducing microwave transitions and detecting the effect of microwaves.

This technique has been applied to: (i) semiconductors (Cavenett 1981), where it was found that the recombination rate of excess carriers can be influenced by changing their spin orientation (or that of paramagnetic recombination centre in a resonant microwave field) and (ii) polymers, to investigate the conductivity mechanism of weakly doped polyacetylene (Frankevich et al 1985).

\section{Concluding remarks}

It is hoped that this article has given a "working knowledge' of the technique of electron paramagnetic resonance as applicable to advanced materials in their solid state. It is apparent that EPR is the 'method of choice' for the range of materials discussed - semiconductors to insulating and conducting polymers to ferroelectric and superconducting ceramics, and structural and optical glasses to optoelectronic and superionic materials - and more, to obtain information about an unpaired electron and its neighbourhood, and to relate it to the macroscopic behaviour. In the future the use of EPR technique for newer materials like multilayer, Langmuir-Blodgett films, ferrofluids to name a few, is being investigated, pushing the 'frontier' of technical parameters - frequency and magnetic field - to unbelievable limits in the quest for accuracy and detail in characterization.

\section{Acknowledgements}

I thank all my colleagues and students whose work has figured in this article. I particularly recall the Late $\mathrm{Dr}$ 
V S Subrahmanyam for many years of collaborative catalytic activity.

\section{List of Symbols}

$a$ Isotropic hyperfine constant;

$A$, hyperfine structure tensor;

$B, \quad$ anisotropic hyperfine constant, sometimes dc magnetic field intensity;

$\tilde{D}, \quad$ Fine structure constant tensor;

$E$, energy, energy level;

$F, f$ line shape function, function in general;

$g, \quad g$-factor, $g$-tensor;

G, Gauss, $g^{2}$-tensor;

$\boldsymbol{g}_{\mathrm{ij}}$, component of $\mathrm{g}$-tensor;

$\boldsymbol{g}_{\mathrm{N}}$, nuclear $\mathrm{g}$-factor;

$\mathscr{H}, \quad$ spin Hamiltonian;

$H, \quad$ dc magnetic field expressed as milli Tesla (mT) or Oersted or Gauss $(10$ Gauss $=1 \mathrm{mT})$;

$H_{1}$, microwave magnetic field;

$H_{\text {ani, }} \quad$ anisotropy field of ferromagnet;

$H_{\text {dem }}$, demagnetization field of non-spherical ferromagnetic sample;

$H_{\mathrm{r}} \quad$ magnetic field at resonance, centre of ESR line;

I, nuclear spin operator, nuclear spin, intensity of ESR spectrum, current;

$K_{u}$, uniaxial anisotropic constant for amorphous ferromagnet;

$l_{\mathrm{ij}}$, components of direction cosine matrix;

$M$, magnetization vector;

$M_{0}$, magnetization along Z-axis;

$M_{\mathrm{s}}$, electron spin quantum number, $m_{\mathrm{I}}$ nuclear spin quantum number;

$N^{+}, N^{-}$, Boltzmann population of levels;

$P$, transition probability, microwave power;

$Q, \quad$ nuclear quadrupole moment, quality factor of cavity;

$r$ position vector;

$R$, alkyl radical;

$s$, saturation factor;

$S, \quad$ electron spin, electron spin operator;

$T_{1}$, spin-lattice relaxation;

$T_{2}, T_{2}^{\prime}, \quad$ spin-spin relaxation time;

$\mathrm{TE}_{011}$, transverse-electric mode of rectangular cavity, numbers refer to components along cavity dimensions $(a, b c)$;

$U, V, W$, coefficients of $g^{2}(\theta)$ function;

$x$, coordinate axis;

$X, \quad$ test sample, principal direction;

$y$, coordinate axis;

$Y$, principal direction;

$z, \quad$ coordinate axis;
$Z$, circuit impedance, principal direction;

$\alpha, \beta, \quad$ label for protons in a polymer;

$\gamma, \quad$ magnetogyric ratio;

$\delta$, skin depth;

$\Delta H_{\mathrm{pp}}$, peak-to-peak width of the first derivative ESR signal;

$\varepsilon$, dielectric constant;

$\eta$, filling factor of cavity;

$\theta$, angle between two vectors;

$\lambda$, spin-orbit coupling constant, microwave penetration depth, Gilbert damping factor in ferromagnetic resonance;

$\mu_{\mathrm{B}}$, Bohr magneton;

$\mu_{\mathrm{e}}, \quad$ electronic magnetic moment;

$\mu_{\mathrm{N}}$, nuclear magneton;

$\nu, \quad$ microwave frequency, ENDOR transition frequency;

$\chi$, magnetic susceptibility.

\section{References}

Abragam A and Bleaney B 1989 in Electron paramagnetic resonance of transition ions (New York: Dover)

Alger R S 1968 in Electron paramagnetic resonance techniques and applications (New York: Interscience)

Atherton N M 1973 in Electron spin resonance (Chichester: Ellis Horwood) Ch. 1

Badalyan A G and Zhitnikov R A 1985 Sov. Phys. Solid State $27 \quad 1774$

Bagguley D M S and Griffiths J H E 1950 Proc. Phys. Soc. A201 366

Balagopala Krishna C and Rajasekharan M V 1990 Phys. Rev. B42 7794

Balaya P and Sunandana C S 1990 Solid State Ionics 40/41 770

Balaya $\mathrm{P}$ and Sunandana C S 1990 Recent advances in fast ion conducting materials and devices (eds) B V R Chowdari et al (Singapore: World Scientific) p. 535; 1994 J. Phys. Chem. Solids $\mathbf{5 5} 39$

Bartl A, Frohner J, Znzok R and Roth S 1992 Synth. Metals 51197

Bartl A, Frohner J and Roth S 1993 Synth. Metals 55-57 613

Bensimon Y et al 1992 J. Non-Cryst. Solids 149218

Beranov P G and Badalyan A G 1993 Solid State Commun. 85 987

Berger R and Haddad M 1991 Phys. Status Solidi b163 463

Bhagat S M 1973 Resonance methods of magnetic materials, Part II (ed.) R F Bunshah (New York: Wiley) Vol. II

Bhagat S M, Manheimer M A and Moorjani K 1987 Key Engg. Mater. 13-15 641

Bhat S V, Ganguly P, Ramakrishnan T V and Rao C N R 1987 J. Phys. C: Solid State 20 L559

Bishop S G, Strom U and Taylor P C 1977 Phys. Rev. B15 2278

Bowman R C Jr., Venturini E L and Witt S N 1987 J. Vac. Sci. Tech. A5 3171 and references therein

Bravo D, Martin A and Lopez F J 1993 Solid State Commun. 86281 and references therein 
Bruker Almanac 1993 Bruker spectrospin, Zurich

Byszewski P, Jablonski R and Kolesnik S 1992 Solid State Commun. 841111

Campbell D and Peterlin A 1968 Polym. Letts 6481

Canham L T 1990 Appl. Phys. Lett. 571046

Caplan P I, Poindexter E H, Deal B E and Razouk R R 1977 J. Appl. Phys. 505847

Carter F L (ed.) 1982/1987 Molecular electronic devices I, II (New York: Dekker)

Caurant D, Gourier D, Vivien D and Prasson M 1993 J. Appl. Phys. 731657

Cavenett B C 1981 Adv. Phys. 30475

Chary K V R, Mahipal Reddy B, Nag N K, Subramanyam V S and Sunandana C S 1984 J. Phys. Chem. 882622

Chen J, Chung T C, Moras F and Heeger A J 1985 Solid State Commun. $\mathbf{5 3} 757$

Commoner B, Townsend J and Pake G E 1954 Nature 174689

Das R et al 1986 Pramana-J. Phys. 27661

Deville A et al 1993 Phys. Rev. B47 2840

Duke C B and Fabysh T T 1978 J. Appl. Phys. 49315

Dikanev S A and Ashtashkin A V 1978 in Principles of magnetic resonance (ed.) A J Hoff (Amsterdam: Elsevier) Ch. 2, p. 59

Doyle W T 1962 Rev. Sci. Instrum. 33118

Durny R 1980 J. Non-Cryst. Solids 41273

Einstein A and Ehrenfest P 1922 Z. Phys. 1131

Evora C and Jaccorino V 1977 Phys. Rev. Lett. 391554

Feher G 1959a Phys. Rev. 1141219

Feher G 1959b Bell. Syst. Tech. J. 36449

Frankevich E L et al 1985 Phys. Status Solidi B132 283

Freed J H, Budil D K, Earle K A and Lynch W B 1989 Advanced EPR (ed.) A J Hott (Amsterdam: Elsevier) Ch. 8, p. 307

Furusawa M and Ikeya M 1990 J. Phys. Soc. Jap. 592340

Fletcher R C, Yager W A, Pearson G L and Merrit F R 1954 Phys. Rev. 95844

Frait $Z$ and Fraitova D 1988 Spin waves and magnetic excitations (eds) A S Borovik-Romonov and S K Sinha (Amsterdam: Elsevier) Ch. 1, p. I

Funsawa M and Ikeya M 1991 Jpn J. Appl. Phys. 60 L1687 Gerlach W and Stem O 1924 Ann. Phys. Leipzig 74673

Gordon J P and Bowers K D 1958 Phys. Rev. Lett. 1301

Gorelkinskii Yu V and Nevinnyi N N 1987a Pisma Zh. Tekh. Fiz. 13105

Gorelkinskii Yu V and Nevinnyi N N 1987b Sov. Tech. Phys. Lett. 1345

Graham R A, Morosin B, Venturini E L and Carr M J 1986 Ann. Rev. Mater. Sci. 16315

Green R E Jr 1990 Ann. Rev. Mater. Sci. 20197

Griffiths J H E 1946 Nature 15867

Griscom D L $1973 / 74$ J. Non-Cryst. Solids 13251

Griscom D L 1980 J. Non-Cryst. Solids 40211

Griscom D L, Stapelbroek M and Webb M J $1986 \mathrm{~J}$. Non-Cryst. Solids 41329

Gunter P and Heiguard J P 1988/1989 Photorefractive materials and their applications, in Topics 61 and 62 in applied physics (Berlin: Springer Verlag) Vols 1 and II

Hagen W R 1992 Adv. Inorg. Chem. 38165

Hahn E L 1950 Phys. Rev. 80580

Haller E E and Falicov L M 1978 Phys. Rev. Lett. 41192

Hamanone K et al 1974 J. Polymer Sci. A12 1189
Harper W R 1964 Contact and fictional electrification (Oxford: Clarendon)

Hebard A et al 1991 Nature 350600

Henderson B and Gorrison A K 1973 Adv. Phys. 22423

Heniker J 1962 Nature 196474

Holczer K, Boucher J P, Devreux F and Nechtschien M 1981 Phys. Rev. B23 1051

Holton W C and Blum M 1962 Phys. Rev. 12589

Humphries G M K and McConnell H M 1982 Methods of experimental physics (eds) G Ehrenstein and H O Lecar (New York: Academic) Vol. 20, Ch. 2, p. 53

Hyde J S and Froncisz W 1989 Advanced EPR, applications in biology and biochemistry (ed.) A J Hott (Elsend) Ch. 7, p. 227

Ikeya M 1991 Annu. Rev. Mater. Sci. 2145

Ikeya M 1993 in New applications of electron spin resonance, dating, dosimetry and microscopy (Singapore: World Scientific Pub.)

Ikeya M, Megvo K, Miyamaru H and Ishii H 1991 Appl. Mag. Res. 2663

Isoya J et al 1990 Phys. Rev. B41 3905

Iwasaki M et al 1971 J. Chem. Phys. 541839

Izumi $\mathrm{T}$ et al 1993 Thin Solid Films 228285

Janes R, Liu R-S, Edwards P P, Stevens A D and Symons M C R 1991 J. Chem. Soc. Faraday Trans. 871209

Jones R, Janes R, Armstromy R, Pyper N C, Edward P P, Keeble D J and Harrison M R $1990 \mathrm{~J}$. Chem. Soc. Faraday Trans. 86675

Kaul S N and Babu P D 1992 Phys. Rev. B45 295

Kaul S N and Siriguri V 1992 J. Phys. Condens. Matter 4505

Kausch H H 1978 Polymer fracture (Berlin: Springer Verlag) p. 119

Kawazo H, Watanabe Y and Yamane M 1987 Diffusion Defect Data 53-54 189

Kazanskii V B 1976 Proc. 6th int. congress on catalysis (London: Chemical Society) Vol. 1, p. 50

Kindo K, Honda M, Kohashi T and Date M 1990 J. Phys. Soc. Jap. 592332

Kip A F, Kittel C, Levy D H and Portis A M 1953 Phys. Rev. 911066

Kiss E and Szorenyi T 1977 Amorphous semiconductors (ed.) I K Somogyi (Budapest: Akademiai Kiado) p. 25

Kittel C 1947 Phys. Rev. 71270

Kivelson S and Heeger A J 1985 Phys. Rev. Lett. 553118

Kosaka M et al 1993 Chem. Phys. Lett. 203429

Koschnick F K, Rai. M, Spaeth J M and Eachus R S $1993 \mathrm{~J}$. Phys. Condens. Matter 5733

Koshta A A, Shvachko Yu N, Romanyukha A A and Ustinov V V 1993 Zh. Eksp. Teor. Fiz. 6629

Kubo N O et al 1992 Appl. Phys. Lett. 61940

Kwan C L and Yen T F 1979 Anal. Chem. 511225

Lauterbur P C 1973 Nature 242190

Lebedev Ya S 1963 J. Struct. Chem. (USSR) 422

Lersch W and Michel-Beyerle M E 1989 Advanced EPR (ed.) A J Hoff (Amsterdam: Elsevier) Ch. 19, p. 685

Loubser J H N and Van Ryneweld W P 1966 Nature 211517

Ludwig G W and Woodbury H H 1962 Solid state physics (eds) F Seitz and D Turnbull (New York: Academic) Vol. 13 , p. 223

Mabbs F A and Collison D 1992 Electron paramagnetic resonance 
of $d$ transition metal compounds, in Studies in inorganic chemistry (New York: Elsevier) Vol. 16

Marquardt C I 1976 Appl. Phys. Lett. 28209

Masuda K 1977 Inst. Phys. Conf. Ser. No. 31, (Bristol: Inst. Phys.) Ch. 2, p. 174

McMillan J A 1974 in Systematic materials analysis (ed.) N L Peterson (New York: Academic Press) Ch. 7, Vol. 1, p. 193

McMillan J A and Halpern T 1971 Argonne Natl. Lab. Rep. (Phys.) ANL-7784

Mehran F and Anderson P W 1988 Solid State Commun. 67 1187

Mehring M et al 1993 Proc. workshop on phase separation in cuprate superconductors, Erice, Italy (eds) $\mathrm{K}$ A Muller and C Benedek (Singapore: World Scientific) p. 67

Mims W B 1989 in Advanced EPR (ed.) A J Hoff (Amsterdam: Elsevier) $\mathrm{Ch} .1$, p. 1 and references therein

Mims W B, Nassau K and McGee J D 1961 Phys. Rev. 123 2059

Minami T, Ikeda Y and Tanaka M 1982 J. Non-Cryst. Solids 5215

Miyamura H and Ikeya M 1993 Appl. Radiat. \& Isotopes 44 379

Morigaki K 1988 Amorphous silicon and related materials (ed.) H Fritzsche (Singapore: World Scientific)

Mott N F 1968 J. Non-Cryst. Solids 11

Mott N F 1971 Adv. Phys. 21785

Mrodsky M H and Title R S 1961 Phys. Rev. Lett. 23581

Muller K A et al 1980 Phys. Rev. Lett. 45832

Murata T and Miki T 1993 J. Appl. Phys. 731110

Murthy N R K and Sunandana C S 1992 Solid state ionics: Materials and applications (eds) B V R Chowdari et al (Singapore: World Scientific) p. 357

Myers S M et al 1992 Rev. Mod. Phys. 64559

Nechtschien M, Devreux F, Genoud F, Gugleelumi $M$ and Holezer K 1983 Phys. Rev. B27 61

Ohno K 1982 J. Magn. Reson. 50 145

Ohta H et al 1992 J. Phys. Soc. Jap. 612921

Pakulis E J and Jeffries C D 1981 Phys. Rev. Lett. 451859

Pantelides S T 1988 Amorphous silicon and related materials (ed.) H Fritzsche (Singapore: World Scientific) p. 541

Pfenninger S, Forrer J, Schweiger A and Weiland Th 1988 Rev. Sci. Instrum. 59752

Pilbrow J R 1990 Transition ion electron paramagnetic resonance (Oxford, London, New York: Clarendon Press)

Poole C P 1983 in Electron spin resonance, A comprehensive treatise on experimental techniques (New York: Interscience) 2nd ed.

Possenriede E 1989 J. Phys. Condens. Matter 17267

Rabi I I et al 1939a Phys. Rev. 55526

Rabi I I et al 1939b Phys. Rev. 56728

Rakvin B et al 1990 Phys. Rev. B41 769

Ramasastry C and Sunandana C S 1976 J. Magn. Res. 2387

Ranby B and Rabek J F 1977 ESR spectroscopy in polymer research (Berlin: Springer Verlag)

Ranby B and Rabek J F 1977 ESR spectroscopy in polymer research (Berlin: Springer Verlag) Ch. VIII

Rao K J and Rao B G 1985 Proc. Indian Acad. Sci. (Chem. Sci.) 95169

Reichart J F and Pereli J 1966 Rev. Sci. Instrum. 37426

Rice M J 1979 Phys. Lett. A71 152
Rodriguez S 1987 Physica B146 212

Roitsin A B and Maevskii V M 1989 Sov. Phys. Usp. 32891

Roth L M, Lax B and Zwerdling S 1959 Phys. Rev. 11490

Rytz D et al 1990 J. Opt. Soc. Am. B7 2245

Sahyun M R V 1992 Chemtech 418

Sakaguchi M et al $1988 \mathrm{~J}$. Polym. Sci. Part B, Polym. Phys. 261307

Sakaguchi M et al 1989 Macromolecules 221277 and references therein

Sakaguchi M, Shimada M and Kashiwabara H 1990 Macromolecules 235038

Sakaguchi M, Yamakuchi T, Shimada S and Hon Y 1993 Macromolecules 262612

Sakita S and Fujii I 1991 Jpn J. Appl. Phys. 30 L1489

Sands R H 1955 Phys. Rev. 991222

Sariffici N S, Smilowitz L, Heeger A J and Wudl F 1992 Science 2581474

Schonland D S 1959 Proc. Phys. Soc. 73788

Scheffler M 1987 Lattice relaxations at substitutional impurities in shallow impurity centre in semiconductors (eds) A Boulder and R Resta (Amsterdam: North Holland) p. 171

Schweiger A 1989 Advanced EPR (ed.) A J Hoff (Amsterdam: Elsevier) Ch. 6, p. 243

Scripsick M P 1993 J. Appl. Phys. 731114

Shimada H, Hon Y and Kashiwabara H 1988 Macromolecules 212107

Shirakawa H and Ikeda S 1971 Polym. J. 2231

Sieverts E G and Ammerlaan C A J 1977 Inst. Phys. Conf. Ser. No. 31, Ch. 2, p. 213

Simon S and Al Nicula 1983 J. Non-Cryst. Solids 5723

Slichter C P 1955 Phys. Rev. 99479

Slichter C P 1978 in Principles of magnetic resonance (Berlin: Springer Verlag) 2nd ed., p. 38

Sozzani F, Di Silvestro G and Gervasini A 1986 J. Polym. Sci. Part A: Polym. Chem. 24815

Son N T, Kustov V E, Gregorkiewicz T and Ammerlaan C A J 1992 Phys. Rev. B46 4544

Spaeth J M and Koschnick F K 1991 J. Phys. Chem. Solids 521

Spano M L and Bhagat S M 1981 J. Magn. Magn. Mater. 24 143 and references therein

Stallinga P, Gregorkiewicz T, Ammerlaan C A J and Gorelkinskii Yu V 1993 Phys. Rev. Lett. 71117

Stern O 1921 Z. Phys. 7249

Stone T J, Buckman T, Nordio P L and McConnell H M 1965 Proc. Natl. Acad. Sci. USA 541010

Stutzmann M and Street R A 1985 Phys. Rev. Lett. 541836

Stutzmann M and Biegelson D K 1989 Phys. Rev. B40 9834

Stutzmann M, Biegelson D K and Street R A 1987 Phys. Rev. B35 5666

Sukei M, Eaton S S and Eaton G R 1993 Appl. Radiat. \& Isotopes 44377

Sunandana C S 1975 in ESR studies on some X-irradiated and doped sulphates, Ph.D. Thesis, Indian Institute of Technology, Madras

Sunandana C S 1978 Phys. Status Solidi A48 K19

Sunandana C S 1987 Key Engg. Mater. 13-15 623

Sunandana C S and Bhatnagar A K $1984 \mathrm{~J}$. Phys. C: Solid State Phys. 17467

Sunandana C S and Rao K S 1985 Phys. Status Solidi A90 681 
Taylor P C, Baugher J F and Kriz H M 1975 Chem. Rev. 75 203

Tinkham M 1996 Introduction to superconductivity (New York: McGraw Hill) 2nd ed.

Title R S and Chandrasekhar G V 1976 Solid State Commun. 20405

Vanheusden K and Stesmans A 1993 Appl. Phys. Lett. 222405

Van Kemp R, Sieverts E G and Ammerlaan C A J 1987 Phys. Rev. B36 3528

Van Veen G 1978 J. Magn. Reson. 3091

Venugopal Rao G, Sunandana C S and Bhatnagar Anil K 1992 J. Phys. Condens. Matter 41373

Von Bardeleben H J et al 1992 Mater. Res. Symp. Proc. (eds) G L Witt, R Calavu, U Mishra and U Weber (Philadelphia: Mater. Res. Soc.) Vol. 241, p. 69

Warren W L et al 1993 Appl. Phys. Lett. 62482 and references therein

Watanabe I, Inakagi Y and Shimizu T 1978 J. Non-Cryst. Solids 27295

Watkins G D, Kleverman M, Thilderkvist $A$ and Grimmeiss H G 1991 Phys. Rev. Lett. 671149

Webb E and Alexander H 1977 Inst. Phys. Conf. Ser. No. 31, Ch. 2, p. 266

Weeks R A 1956 J. Appl. Phys. 271376

Wertz J E and Bolton J R 1972 in Electron spin resonance, elementary theory and practical applications (New York: McGraw Hill)

Whitmore M H 1993 J. Chem. Phys. 983656

Wilmshurst T H 1967 in Electron spin resonance spectrometer (London: Hilger)

Winkler P F, Kleppner D, Myint T and Walther F G 1972 Phys. Rev. A5 83

Yasaitis E L and Smaller B 1953 Phys. Rev. 921068

Yokomichi H and Morigaki K 1987 Solid State Commun. 63 629

Yokomichi H and Morigaki K 1993 Solid State Commun. 85 759

Yokomichi H, Takakuki H and Kondo M 1993 Jap J. Appl. Phys. 32 L365

Yordanov N D (ed.) 1991 in Electron magnetic resonance of disordered systems (EMARDIS 91) (Singapore: World Scientific)

Zakrevskii V A et al 1968 Sov. Phys. Solid State 101341

Zavoiskii E K 1944 Phys. Inst. Acad. Sci., Moscow
Zhilinskaya E A, Valeev N K H and Oblasoe A K $1991 J$. Non-Cryst. Solids 13676

Zhong Y C and Pilbrow J R 1991 J. Magn. Res. 93447

Zunger A 1986 Solid state physics (eds) F Seitz, D Turnbull and $H$ Ehrenreich Vol. 39, p. 275

Note added in proof. The following developments have been most noteworthy - ever since this article was submitted. Among the techniques, electrical detection of EPR (B Stich et al $1995 \mathrm{~J}$. Appl. Phys. 77 1546), that measures the microwave/rf induced change of conductivity of semiconductors containing shallow and deep defects, offers new possibilities for the study of point defects. Such a study on a Si diode (Z Xiang and D J Miller 1995 J. Appl. Phys. 78 4895) has identified broken bonds in a vacancy cluster acting as recombination centres. Bhat et al (S V Bhat, A Anand and Rajiv Bhat 1997 Solid State Physics (India) 40C 62) have developed a spectrometer for unmodulated high-power rf absorption studies in high-temperature superconductors. Exciting applications include: study of spin-Peierls transitions in $\mathrm{Cu}_{1-x} \mathrm{Zn}_{x} \mathrm{GeO}_{3}$ (P Fronzes et al 1997 Phys. Rev. B56 7827), low-temperature phase transitions in $\mathrm{CuO}$ by a DPPH probe (A M Suvarna and C S Sunandana 1997 Physica C276 65), defects in diamond films (A K Sikder et al 1997 Solid State Physics (India) 40C 435), $\mathrm{Ni}^{+}$in $\mathrm{AgGaSe}_{2}$ (L E Halliburton et al $1996 \mathrm{~J}$. Appl. Phys. 79 556), Cr-doped fluorochloro- and fluorobromozirconate glasses (J L Martinez et al 1997 J. Phys. Cond. Matt. 9 9175) and studies of dangling bonds in porous silicon (Y Xiao et al 1994 J. Appl. Phys. 76 1759; R Laiho and L S Vlasenko $1995 \mathrm{~J}$. Appl. Phys. 78 2857), besides a very interesting study of an oxygen defect centre associated with red photoluminescence from freshly etched and oxidized porous silicon (S M Prokos and W E Carlos 1995 J. Appl. Phys. 78 2671). A comprehensive review of EPR in semiconductors has appeared (W Gehlhoff, M Hohne and J Schmidt 1992 in Hyperfine interaction of defects in semiconductors (ed.) G Langouche (Amsterdam: Elsevier) Chap. 5, p. 217). 